\title{
Dinâmica da equação de Schrödinger com potencial delta de Dirac em espaço com peso
}

\author{
Ânderson da Silva Vieira
}

TESE APRESENTADA

AO

Instituto DE MatemáticA E Estatística

DA

Universidade De SÃo PaUlo

PARA

OBTENÇÃO DO TÍTULO

$\mathrm{DE}$

DOUTOR EM CIÊNCIAS

Programa: Matemática

Orientador: Prof. Dr. Jaime Angulo Pava 


\section{Dinâmica da equação de \\ Schrödinger com potencial delta \\ de Dirac em espaço com peso}

Esta tese trata-se da versão original

do aluno Ânderson da Silva Vieira. 


\section{Dinâmica da equação de Schrödinger com potencial delta de Dirac em espaço com peso}

Esta tese contém as correções e alterações sugeridas pela Comissão Julgadora durante a defesa realizada por Ânderson da Silva Vieira em 17/07/2014.

O original encontra-se disponível no Instituto de Matemática e Estatística da Universidade de São Paulo.

Comissão Julgadora:

- Prof. Dr. Jaime Angulo Pava (orientador) - IME-USP

- Prof. Dr. Luiz Augusto Fernandes de Oliveira - IME-USP

- Prof. Dr. Sérgio Muniz Oliva Filho - IME-USP

- Prof. Dr. Fábio Matheus Amorin Natali - UEM

- Prof. Dr. Adan José Corcho Fernandez - URFJ 
"Deus não escolhe os capacitados, capacita os escolhidos. Fazer ou não fazer algo só depende de nossa vontade e perseverança." Albert Einsten 
Dedicado à minha saudosa Vó Quitéria 


\section{Agradecimentos}

Em primeiro lugar agradeço a Deus por ter me dado oportunidade, saúde, força e sabedoria para que eu pudesse concluir esse trabalho.

Ao meu orientador, Prof. Jaime, pelo desafio oferecido e pelo nosso tempo de estudo. O Jaime é um pesquisador que tem a sabedoria de ver além do horizonte, além de transbordar conhecimento. Serei eternamente grato ao seu companheirismo nessa fase profissional e às agregações do saber matemático em minha carreira. Ao Prof. Luiz, que esteve ao meu lado para orientar-me nos primeiros passos dessa pesquisa. Ele é um pesquisador que gosta de desafios e sabe de tudo um pouco. Isso me motiva a continuar pesquisando.

À minha amada esposa, Marcela, que no seu silêncio me deu forças e fez pedidos para que eu concluísse mais uma etapa importante. Seu companheirismo, sua fidelidade e sua preocupação em me fazer feliz, faz com que eu renove cada dia o meu compromisso no nosso caminhar juntos. Também a possibilidade de ter as sobrinhas Anna Lívia e Thaila as quais nos amamos em reciprocidade.

Aos meus pais que sempre lutaram e rezaram para que boas oportunidades aparecessem em meu caminho e aos meus irmãos que torcem para que meus objetivos sejam alcançados.

Aos amigos do doutorado, Wilian, Glauce, Rosilene, Eliane e Oscar, pelo belíssimo tempo de convivência e de familiaridade.

Aos meus irmãos de república, Lígia e Júlio, que se tornaram meus amigos eternos.

À minha regente Marcia Hentschel pelo grande acolhimento, pela afeição, pelos ensinamentos e pelas oportunidades de trabalho na música.

À Faculdade Márcio Schenberg onde tive o meu primeiro trabalho como professor univer-

sitário que contribuiu para minha permanência nesse estudo. À Prof. Rita pela afinidade e pelas longas conversas que deram-me perseverança e fé.

Enfim, agradeço aos familiares, amigos e professores, uns bem presentes e outros não tão presentes, mas que formaram uma torcida para que eu alcançasse meus objetivos. 


\section{Resumo}

VIEIRA, Â. S. Dinâmica da equação de Schrödinger com potencial delta de Dirac em espaço com peso. 2014. 112 f. Tese (Doutorado) - Instituto de Matemática e Estatística, Universidade de São Paulo, São Paulo, 2014.

Nesse trabalho, estudamos a equação de Schrödinger não-linear com uma função potencial delta atrativa

$$
i \frac{\partial u}{\partial t}=\left(-\frac{1}{2} \Delta+q \delta(x)\right) u-\lambda|u|^{p} u, \quad(x, t) \in \mathbb{R} \times \mathbb{R},
$$

onde $\lambda \in \mathbb{R}-\{0\}, \Delta=\frac{d^{2}}{d x^{2}}, \delta$ representa a delta de Dirac centrada em zero, $q<0$ e $p>1$. As soluções para essa equação tem uma componente localizada e uma dispersiva. Além de estudar o comportamento das soluções dessa equação em espaços de Sobolev clássicos, mostramos algumas propriedades do grupo unitário $e^{-i t\left(-\frac{1}{2} \Delta+q \delta\right)}$ em espaços $L^{p}, L^{2}$ com peso, Sobolev com peso e assim obtemos alguns resultados de boa colocação local e global das soluções. O ponto central desta tese é mostrarmos a existência de uma variedade invariante centro que irá consistir de órbitas periódicas no tempo bifurcando do ponto $\left(0, E_{0}\right) \in H^{2}(\Omega) \times \mathbb{R}$, onde $E_{0}$ é o autovalor simples (isolado) do operador $H_{q}$. Para isto, usamos específicas propriedades da parte do espectro contínuo da solução em espaços de Sobolev com peso. Além disso, mostramos que toda solução com dado inicial pequeno vai se aproximar de uma órbita periódica particular da variedade invariante centro quando $t \rightarrow \pm \infty$. Afim de obtermos os mesmos resultados, sem usar os espaços de Sobolev com peso, finalizamos com uma aplicação mudando a não-linearidade; isto é, estudamos o problema de Schrödinger não-linear

$$
i \frac{\partial}{\partial t} u=\left(-\frac{1}{2} \Delta+q \delta(x)\right) u+f(x,|u|) \frac{u}{|u|},
$$

onde $f$ é de valor real e satisfaz certas condições sobre regularidade e crescimento como uma função de $u$ e tem decaimento quando $x \rightarrow \pm \infty$.

Palavras-chave: Equação de Schrödinger não-linear, Potencial Delta de Dirac, Variedade Invariante Centro, Espaços $L^{p}$ e de Sobolev com peso. 


\section{Abstract}

\section{VIEIRA, Â. S. Dynamics of Schrödinger equation with Dirac delta potential in weighted} space. 2014. 112 f. Tese (Doutorado) - Instituto de Matemática e Estatística, Universidade de São Paulo, São Paulo, 2014.

In this work, we study the nonlinear Schrodinger equation with an attractive delta function potential

$$
i \frac{\partial u}{\partial t}=\left(-\frac{1}{2} \Delta+q \delta(x)\right) u-\lambda|u|^{p} u, \quad(x, t) \in \mathbb{R} \times \mathbb{R},
$$

where $\lambda \in \mathbb{R}-\{0\}, \Delta=\frac{d^{2}}{d x^{2}}, \delta$ is Dirac delta centered at zero, $q<0$ e $p>1$. The solutions to this equation have a localized and a dispersive component. In addition to studying the behavior of solutions of this equation in classical Sobolev space, we show some properties for the unitary group $e^{-i t\left(-\frac{1}{2} \Delta+q \delta\right)}$ in $L^{p}$, weighted $L^{2}$ and Sobolev spaces and so we get some results of local and global well-posedness of solutions. The central theme this thesis is to show the existence of a center invariant manifold, which will consist of time-periodic orbits bifurcating from the point $\left(0, E_{0}\right) \in H^{2}(\Omega) \times \mathbb{R}$, where $E_{0}$ is simple eigenvalue (isolated) of operator $H_{q}$. For this, we use specifics properties of the spectrum continuous part of the solution in weighted Sobolev space. Furthermore, we show that every solution with small initial data will approach a time-periodic orbit particular in center invariant manifold as $t \rightarrow \pm \infty$. In order to obtain the same results without using weighted Sobolev spaces, we finished with an application changing the nonlinearity; that is, we study the nonlinear problem

$$
i \frac{\partial}{\partial t} u=\left(-\frac{1}{2} \Delta+q \delta(x)\right) u+f(x,|u|) \frac{u}{|u|},
$$

where $f$ is real-valued and satisfies certain conditions of regularity and growth as a function of $u$ and it has decay as $x \rightarrow \pm \infty$.

Keywords: Nonlinear Schrödinger Equation, Delta Dirac potential, Center Invariant Manifold, Weighted $L^{p}$ and Sobolev Spaces. 


\section{Sumário}

Lista de Figuras

Notação $\quad$ vii

1 Introdução 1

2 Pré-requisitos $\quad 9$

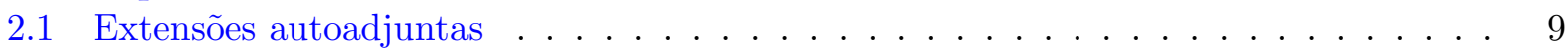

2.2 Equação de Schrödinger linear . . . . . . . . . . . . . . . . . . . . . . . . 14

2.3 Bifurcação com o núcleo unidimensional . . . . . . . . . . . . . . . . . 16

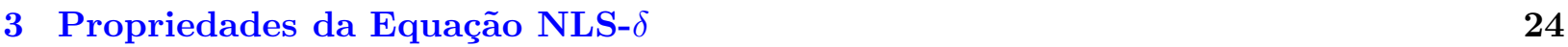

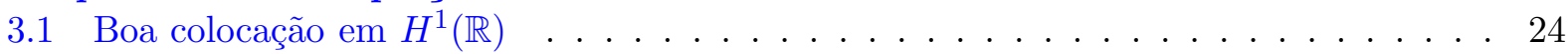

3.2 Fórmula explícita para o grupo gerado por $H_{q}$ e a projeção espectral contínua . . . . 31

3.3 Projeções e as componentes pontuais e contínuas associadas à equação (3.1) . . . . . 35

3.4 A equação linear associada a NLS- $\delta$ e estimativas dispersivas . . . . . . . . . . . . 36

4 Caracterização da Variedade Centro local $\mathcal{W}_{\mu}^{p} \quad 38$

4.1 Existência de uma curva suave peak-standing waves . . . . . . . . . . . . . 38

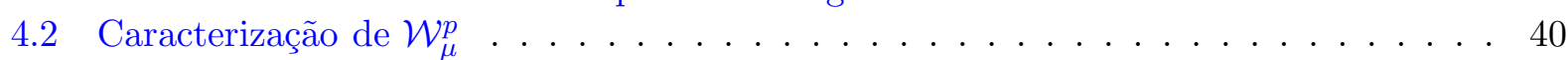

$5 \quad$ A NLS- $\delta$ em espaço de Sobolev com peso 48

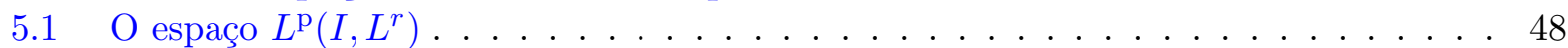

5.2 Espaços de Sobolev com peso . . . . . . . . . . . . . . . . . . . . . . 49

5.3 Propriedades das soluções no espectro contínuo . . . . . . . . . . . . . . . 58

6 Aproximação à Variedade Centro e convergência para uma órbita $\quad 65$

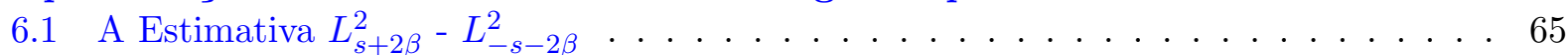

6.2 Aproximação à Variedade Centro . . . . . . . . . . . . . . . . . . . . 76

6.3 Convergência para uma órbita periódica . . . . . . . . . . . . . . . . 82

7 Aplicação à equação de Schrödinger com um ponto de interação $\quad 85$

7.1 NLS- $\delta$ com não linearidades gerais . . . . . . . . . . . . . . . . 85

7.2 Estabilidade assintótica para bound-states não-lineares associados a (7.8) $\ldots$. . . . 88

7.3 Considerações . . . . . . . . . . . . . . . . . . . . . . . 91

8 Futuros Trabalhos $\quad 92$

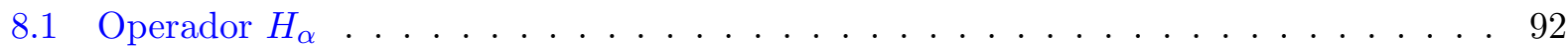

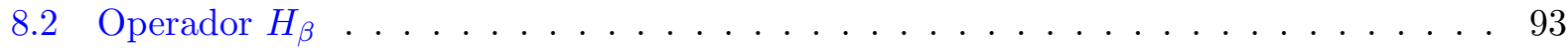

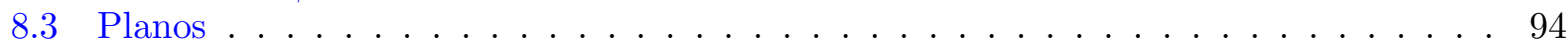


A Soluções da NLS- $\delta \quad \mathbf{9 5}$

A.1 Equação solução de (1.3) . . . . . . . . . . . . . . . . . . . . . . 95

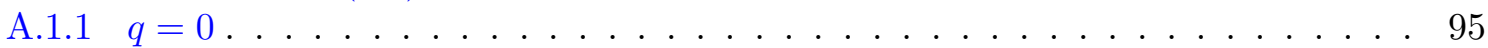

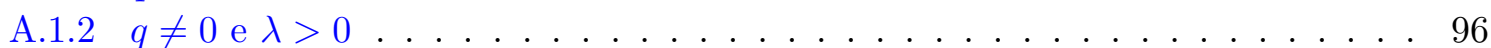

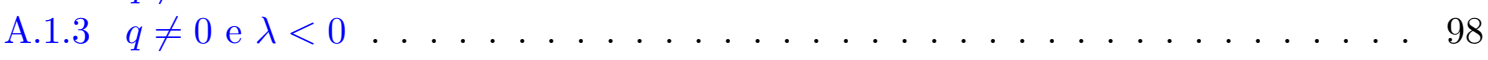

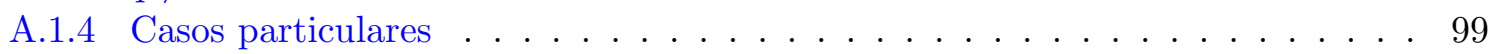

$\begin{array}{ll}\text { B Contas do Teorema 6.1.1 } & 101\end{array}$

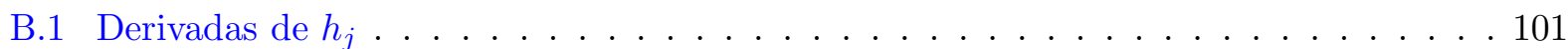

B.2 Limitação em $t$ no $1^{\circ}$ Caso . . . . . . . . . . . . . . . . . . . . . . . . . . . . 104

B.3 Integrais finitas do primeiro caso . . . . . . . . . . . . . . . . . . . . 105

B.4 Lema de Schur . . . . . . . . . . . . . . . . . . . . . . . . . 105

$\begin{array}{lr}\text { Referências } & 107\end{array}$

$\begin{array}{lr}\text { Índice Remissivo } & 111\end{array}$ 


\section{Lista de Figuras}

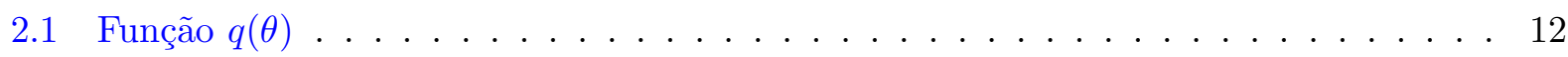

2.2 Diagrama de Bifurcação . . . . . . . . . . . . . . . . . . . . 20

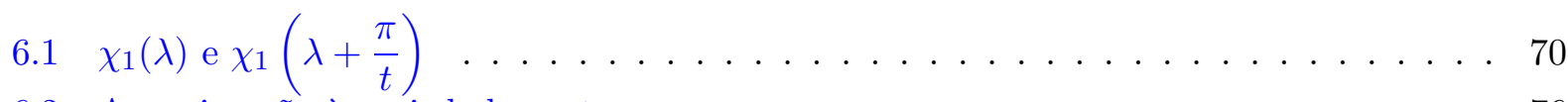

6.2 Aproximação à variedade centro . . . . . . . . . . . . . . . . . 76

A.1 Perfil com $\lambda>0$ e $q>0 \ldots \ldots \ldots \ldots \ldots \ldots \ldots \ldots$

A.2 Perfil com $\lambda>0$ e $q<0 \ldots \ldots \ldots \ldots \ldots \ldots \ldots$

A.3 Perfil com $\lambda>0$ e $q>0$ ou $q<0 \ldots \ldots \ldots \ldots \ldots$ 


\section{Notação}

Durante esta tese estaremos utilizando as seguintes notações:

- $\mathcal{L}(A, B)$ é o Espaço dos Operadores Limitados de $A$ em $B$;

- $\mathscr{N}(A)$ é o Núcleo do operador linear $A$;

- $\mathscr{R}(A)$ é a Imagem do operador linear $A$;

- $\mathscr{D}(A)$ é o Domínio do operador linear $A$;

- $A^{*}$ é o Adjunto do operador linear $A$;

- $A^{\perp}$ é o espaço ortogonal ao espaço $A$;

- $A \subset B$, visto $A$ e $B$ como operadores, representa que $B$ é uma extensão de $A$;

- $P_{p}$ é a projeção sobre o espectro pontual;

- $P_{c}$ é a projeção sobre o espectro contínuo;

- $\Delta=\frac{d^{2}}{d x^{2}}$

- $H_{q}=-\frac{1}{2} \Delta+q \delta(x)$;

- $\Omega=\mathbb{R}-\{0\}$

- $E_{0}$ é o autovalor simples do operador $H_{q}, q<0$;

- $\|\cdot\|_{p}$ norma no espaço de Lebesgue $L^{p}(\mathbb{R})$;

- $\psi_{0}$ é a autofunção normalizada associada ao autovalor $E_{0}$;

- $\left[\psi_{0}\right]$ é o subespaço gerado pela autofunção $\psi_{0}$;

- $\sigma_{p}(A)$ espectro ponto do operador $A$;

- $\langle x\rangle^{\sigma}=\sqrt{\left(1+|x|^{2}\right)^{\sigma}}$;

- $\|f\|_{L_{\sigma}^{2}}=\left(\int\left(1+|x|^{2}\right)^{\sigma}|f(x)|^{2} d x\right)^{\frac{1}{2}}=\left(\int\left|\langle x\rangle^{\sigma} f(x)\right|^{2} d x\right)^{\frac{1}{2}}$;

- $\mathcal{S}(\mathbb{R})$ é o espaço de Schwartz das funções decrescendo rapidamente em $\mathbb{R}$; 
- A transformada de Fourier $\hat{f}$ de uma função $f \in \mathcal{S}(\mathbb{R})^{\prime}$ é

$$
\hat{f}(\xi)=\frac{1}{2 \pi} \int_{-\infty}^{\infty} f(x) e^{-i x \xi} d x
$$

- Espaço de Sobolev de ordem $s \in \mathbb{R}$

$$
H^{s}(\mathbb{R})=\left\{u \in \mathcal{S}(\mathbb{R})^{\prime}:(I-\Delta)^{\frac{s}{2}} u \in L^{2}\right\},
$$

com norma $\|f\|_{H^{s}}^{2}=\int_{\mathbb{R}}(1+|\xi|)^{s}|\hat{f}(\xi)|^{2} d \xi ;$

- Produto interno no espaço $H^{1}(\mathbb{R})$ :

$$
(\phi, \psi)=\int_{\mathbb{R}} \phi(x) \overline{\psi(x)} d x
$$

- Espaço de Sobolev com peso

$$
W_{m}^{k, p}=W_{m}^{k, p}(\mathbb{R})
$$

é o espaço de Banach complexo com a norma

$$
\|\phi\|_{W_{m}^{k, p}}=\left(\sum_{\alpha=0}^{k}\left\|\partial^{\alpha} \phi\right\|_{p}^{p}+\sum_{\alpha=0}^{m}\left\|x^{\alpha} \phi\right\|_{p}^{p}\right)^{\frac{1}{p}} .
$$

Quando $p=2$, denotamos $H_{m}^{k}=H_{m}^{k}(\mathbb{R})=W_{m}^{k, 2}(\mathbb{R})$.

- Se $X$ e $Y$ são dois espaços, definimos a norma em $X \cap Y$ por

$$
\|f\|_{X \cap Y}=\max \left(\|f\|_{X},\|f\|_{Y}\right) .
$$




\section{Introdução}

A teoria quântica teve seu surgimento em 1900 quando o famoso e premiado físico alemão Max Planck apresentou seu revolucionário conceito de um quantum e esse evento é considerado o ponto divisor entre a física clássica e moderna (física quântica). Até então, acreditavam-se que dentro da física estudada, não era possível desenvolver novas teorias; ou seja, apenas seria possível preencher as lacunas dos estudos anteriores. Entretanto, Planck vem e derruba esse paradigma apresentando novos estudos que dão início à Teoria da Mecânica Quântica e neste período aparecem as descobertas do raio-x, o elétron e a radioatividade.

Uma equação que tem um papel central na Teoria da Mecânica Quântica é a equação de Schrödinger. A equação descreve a evolução de uma partícula no tempo. Se as partículas no sistema quântico são átomos, moléculas ou partículas subatômicas, a equação tem papel análogo à $2^{\text {a }}$ Lei de Newton na Mecânica Clássica. A equação de Schrödinger foi formulada no final de 1925 pelo físico austríaco Erwin Schrödinger e publicada em 1926. (veja [39])

A equação de Schrödinger pode ter duas formas, uma que depende do tempo e a outra não (Veja [41]). O exemplo mais famoso quando depende do tempo é a equação para uma partícula movendo-se um um campo elétrico, mas não magnético, a saber,

$$
i \hbar \frac{\partial}{\partial t} \Psi(\mathbf{r}, t)=\left[\frac{-\hbar^{2}}{2 m} \Delta+V(\mathbf{r}, t)\right] \Psi(\mathbf{r}, t),
$$

onde $\hbar$ é a constante de Planck, " $m$ " é a massa da partícula, $V$ sua energia potencial, $\Delta$ o Laplaciano e $\Psi$ é uma função onda, que nesse contexto é chamada função onda posição espaço ou position-space wave function. Quando não depende do tempo obtemos o problema de autovalores

$$
E \Psi(\mathbf{r})=\left[\frac{-\hbar^{2}}{2 m} \Delta+V(\mathbf{r})\right] \Psi(\mathbf{r}), \quad E \in \mathbb{C} .
$$

No nosso estudo, estamos trabalhando em um caso particular da equação do tipo (1.1); isto é, no caso quando o potencial $V$ não depende do tempo. Por outro lado, temos autores que trabalham com o caso em que o potencial também depende do tempo (veja [27], [46], [7])

No artigo de Pillet e Wayne em [35] foi estudado o comportamento das soluções com dado inicial pequeno para a equação de Schrödinger não-linear

$$
i \phi_{t}=(-\Delta+V) \phi+\lambda|\phi|^{m-1} \phi, \quad(x, t) \in \mathbb{R}^{n} \times \mathbb{R},
$$


com $\lambda \in \mathbb{R}, n \geq 3$ e $\Delta$ o Laplaciano. O potencial $V$ é escolhido tal que o operador $-\Delta+V$ possui apenas um autovalor negativo simples e seu espectro absolutamente contínuo é a semirreta positiva. Pillet e Wayne mostraram a existência de uma variedade invariante centro $\mathcal{W}_{\mu}^{p}$, a qual consiste de órbitas periódicas no tempo, e finalizaram mostrando que toda órbita próxima de $\mathcal{W}_{\mu}^{p}$, se aproximarão de $\mathcal{W}_{\mu}^{p}$ quanto $t \rightarrow \pm \infty$ (veja Figura 6.2). A equação (1.2) também foi estudada por Soffer e Weinsten em [42, 43] e por Rose e Weinstein em [38].

Motivados pelo problema descrito acima, consideraremos o potencial $V$ como sendo a distribuição delta de Dirac; mais especificamente, $V(x)=q \delta(x)$, com $q \in(-\infty, 0)$ e $x \in \mathbb{R}$. Quando $q \neq 0$, a função potencial tem duas classificações: se $q<0$, dizemos que a função potencial é atrativa; se $q>0$, a função potencial é repulsiva. Este tipo de potencial também foi considerado pelos autores Datchev e Holmer em [12], Holmer, Marzuola e Zworski em [26] e Goodmam, Holmes e Weinstein em [20] que também estudaram a equação de Schrödinger (1.2). Tanto Datchev et al. em [12] quanto Holmer e Holmer em [26], usam a teoria de espalhamento para obter uma expressão para o grupo associado ao operador $H_{q}$ e Goodmam et al. em [20] mostram a boa colocação em $H^{1}(\mathbb{R})$ usando a teoria de operadores de onda.

Apresentamos a seguir o problema que será considerado em todo o trabalho e tem como objetivo investigar o comportamento assintótico das soluções do modelo de tipo Schrödinger (NLS$\delta)$

$$
i \frac{\partial u}{\partial t}=H_{q} u-\lambda|u|^{p} u, \quad(x, t) \in \mathbb{R} \times \mathbb{R},
$$

onde $\lambda \in \mathbb{R}, H_{q}=-\frac{1}{2} \Delta+q \delta(x)$, com $q<0$ e $\delta=\delta_{0}$ denota a distribuição Delta de Dirac centrada em zero, definido para $\psi \in H^{1}(\mathbb{R})$ como $\left(\delta_{0}, \psi\right)=\psi(0)$. A constante $q$ é chamada de constante de acoplamento. Considerando a equação de Schrödinger não-linear na forma

$$
i \frac{\partial u}{\partial t}=H_{q} u \pm|u|^{p} u
$$

o caso atrativo (focusing) é dado pelo sinal (+) na equação (1.4); enquanto que, o caso repulsivo (defocusing) é dado pelo sinal (-). Assim, a equação (1.3) tem uma não-linearidade atrativa se $\lambda<0$ (focusing) e uma não-linearidade repulsiva se $\lambda>0$ (defocusing).

O modelo (1.3) surge em várias situações físicas, tais como: construção de instrumentos a laser; transmissão de dados em alta velocidade; transporte de ondas de matéria; propagação de raios ópticos em meios não-standard. A equação (1.3), com $q \neq 0$ além dos modelos físicos apresentados acima também aparece em óptica não-linear e condensados de Bose-Einstein. De fato, a distribuição de Dirac é utilizado para modelar uma impureza, ou defeito, localizada na origem. Também neste caso, a equação NLS- $\delta$ (1.3) pode ser vista como um modelo de protótipo para a interação de um soliton amplo com um potencial altamente localizado. Na ótica não-linear, isto modela um soliton de propagação em um meio com um ponto defeito ou a interação de um soliton amplo com um tanto mais estreito em uma fibra bimodal. (veja [2, 13, 32, 33, 40]). No lado experimental, este surge no recente interesse em pontos de impurezas (defeitos) desencadeada pelo grande progresso na construção de legados em nanoescala. 
Notamos que o modelo NLS- $\delta$ com uma impureza na origem no caso repulsivo e/ou no atrativo tem que ser entendido como o seguinte problema de contorno (ver Caudrelier et al. [10])

$$
\left\{\begin{aligned}
i \partial_{t} u(x, t)+\frac{1}{2} u_{x x}(x, t) & =-\lambda|u(x, t)|^{p} u(x, t), \quad x \neq 0 \\
\lim _{x \rightarrow 0^{+}}[u(x, t)-u(-x, t)] & =0 \\
\lim _{x \rightarrow 0^{+}}\left[\partial_{x} u(x, t)-\partial_{x} u(-x, t)\right] & =2 q u(0, t), \\
\lim _{x \rightarrow \pm \infty} u(x, t) & =0 .
\end{aligned}\right.
$$

Ou seja, $u(x, t)$ deve ser a solução da equação de Schrödinger não-linear em $\mathbb{R}^{-}$e $\mathbb{R}^{+}$, contínua em $x=0$, satisfaz a "condição de salto" na origem e anulando-se no infinito. As equações em (1.5) são um caso particular de um modelo mais geral, considerando que a impureza está localizada em $x=0$; na verdade, é a equação de movimento

$$
i \partial_{t} u(x, t)+\frac{1}{2} u_{x x}(x, t)=-\lambda|u(x, t)|^{p} u(x, t), \quad x \neq 0,
$$

com as condições de contorno

$$
\left(\begin{array}{c}
u(0+, t) \\
\partial_{x} u(0+, t)
\end{array}\right)=\alpha\left(\begin{array}{ll}
a & b \\
c & d
\end{array}\right)\left(\begin{array}{c}
u(0-, t) \\
\partial_{x} u(0-, t)
\end{array}\right)
$$

$\operatorname{com}\{a, b, c, d \in \mathbb{R}, \alpha \in \mathbb{C}: a d-b c=1,|\alpha|=1\}$. O caso (1.5) surge para a escolha de $\alpha=a=d=1$, $b=0$, e $c=2 q$.

A equação (1.3) no caso em que $\lambda \neq 0$ tem solução na forma

$$
u_{s}(x, t)=e^{i \omega t} \phi(x), \quad \omega>0
$$

chamada ondas viajantes ou standing waves, com condições específicas sobre o perfil $\phi$. Em nosso estudo, iremos considerar $\phi: \mathbb{R} \rightarrow \mathbb{R}$, tal que $\phi=\phi_{\omega, p}$ é a única solução positiva para a equação e tal que $\phi \in \mathscr{D}\left(H_{q}\right)=\left\{g \in H^{1}(\mathbb{R}) \cap H^{2}(\mathbb{R}-\{0\}) \mid g^{\prime}(0+)-g^{\prime}(0-)=2 q g(0)\right\}$ (veja o sistema (A.3) - página 97).

Para $q=0$, a equação (1.3) é reduzida à equação de Schrödinger não-linear (NLS). Nesse caso, $\operatorname{com} \lambda>0$, temos uma soluçao tipo onda viajante da forma

$$
u_{s_{0}}(x, t)=e^{i \omega t} \phi(x-v t)
$$

onde $v$ é a velocidade da onda e $\phi: \mathbb{R} \rightarrow \mathbb{C}$. Assim, obtemos a solução

$$
u_{s_{0}}(x, t)=\left(\frac{\alpha}{2}\right)^{\frac{1}{p}} e^{i(v x-\varpi t)}\left[\frac{p+2}{\lambda} \operatorname{sech}^{2}\left(p \sqrt{\frac{\alpha}{2}}(x-v t)\right)\right]^{\frac{1}{p}},
$$

onde $\varpi=-\left(v^{2}-\omega\right)$ é a frequência temporal e $\alpha=\omega-\frac{v^{2}}{2}$ (veja a Seção A.1.1). Se $\lambda<0$, obtemos uma solução $\phi$ que satisfaz $\lim _{|\xi| \rightarrow \infty} \phi(\xi)=0$, porém, essa solução tem uma singularidade em $\xi=0$. 
(Ver Observação A.1.1). Como a NLS tem duas simetrias básicas, a saber, translação e rotação, obtemos a órbita gerada pelo perfil $\varphi$

$$
\Omega_{\varphi}=\left\{e^{i \gamma} \varphi(\cdot+y): \gamma \in[0,2 \pi], y \in \mathbb{R}\right\}
$$

que representa uma família a dois parâmetros de soluções para a NLS.

Nosso foco principal é quando $q<0$, porém veremos soluções do tipo (1.6) da equação (1.3) quando $q \neq 0$. Assim, o caso a solucionar é reduzido à equação

$$
-\omega \phi(x)+\frac{1}{2} \phi^{\prime \prime}(x)+\lambda|\phi(x)|^{p} \phi(x)=0, \text { para todo } x \in \mathbb{R}-\{0\} .
$$

(veja as seções do apêndice A.1.2 $(\lambda>0)$ e A.1.3 $(\lambda<0)$ ). Para $\lambda>0$, é bem conhecido que a solução de (1.7) é apresentada pelos autores Fukuizumi, Ohta e Ozawa em [18] e Fukuizumi e Jeanjean em [17] na forma

$$
\phi_{\omega}(x)=\left[\frac{(p+2) \omega}{\lambda} \operatorname{sech}^{2}\left(\frac{p}{2} \sqrt{2 \omega}|x|+\tanh ^{-1}\left(-\frac{q}{\sqrt{2 \omega}}\right)\right)\right]^{\frac{1}{p}},
$$

desde que $\sqrt{2 \omega}>|q|,\left(\omega>\frac{q^{2}}{2}\right)$. A estabilidade de uma onda viajante é definida como segue,

Definição 1.0.1. Dizemos que uma solução onda viajante $e^{i \omega t} \phi_{\omega}$ de (1.3) é estável em $H^{1}(\mathbb{R})$ se para todo $\varepsilon>0$ existe $\eta>0$ tal que se $u_{0} \in H^{1}(\mathbb{R})$ e $\left\|u_{0}-\phi_{\omega}\right\|_{H^{1}}<\eta$, então a solução $u(t)$ de (1.3) $\operatorname{com} u(0)=u_{0}$ existe para todo $t \geq 0$ e satisfaz

$$
\sup _{t \geq 0} \inf _{\theta \in \mathbb{R}}\left\|u(t)-e^{i \theta} \phi_{\omega}\right\|_{H^{1}}<\varepsilon
$$

Caso contrário, $e^{i \omega t} \phi_{\omega}$ é dita ser instável em $H^{1}(\mathbb{R})$.

Nas mesmas hipóteses, segundo Le Coz et al. em [30], $\phi_{\omega}$ tem as propriedades como segue:

- Seja $q<0$ e $\omega>\frac{q^{2}}{2}$.

(a) Se $0<p \leq 4, e^{i \omega t} \phi_{\omega}$ é estável em $H^{1}(\mathbb{R})$ para todo $\omega \in\left(\frac{q^{2}}{2},+\infty\right)$.

(b) Se $p>4$, existe um único $\omega_{1}>\frac{q^{2}}{2}$ tal que $e^{i \omega t} \phi_{\omega}$ é estável em $H^{1}(\mathbb{R})$ para todo $\omega \in\left(\frac{q^{2}}{2}, \omega_{1}\right)$ e instável em $H^{1}(\mathbb{R})$ para todo $\omega \in\left(\omega_{1},+\infty\right)$.

- Seja $q>0$ e $\omega>\frac{q^{2}}{2}$.

(a) Se $0<p \leq 2, e^{i \omega t} \phi_{\omega}$ é instável em $H^{1}(\mathbb{R})$ para todo $\omega \in\left(\frac{q^{2}}{2}, \infty\right)$. 
(b) Se $2<p<4$, existe um $\omega_{2}>\frac{q^{2}}{2}$ tal que $e^{i \omega t} \phi_{\omega}$ é instável em $H^{1}(\mathbb{R})$ para todo $\omega \in\left(\frac{q^{2}}{2}, \omega_{2}\right)$.

(c) Se $2<p<4, e^{i \omega t} \phi_{\omega}$ é instável em $H^{1}(\mathbb{R})$ para todo $\omega \in\left(\omega_{2},+\infty\right)$, onde $\omega_{2}$ é como no item (b) acima.

(d) Se $p \geq 4$, então $e^{i \omega t} \phi_{\omega}$ é instável em $H^{1}(\mathbb{R})$.

Por outro lado, quando $\lambda<0$, a solução de (1.3) de tipo standing-wave é apresentada pelos autores Kaminaga e Ohta em [29] na forma

$$
\phi_{\omega}(x, t)=\left[\frac{(p+2) \omega}{2|\lambda|}\right]^{\frac{1}{p}}\left[\sinh \left(\frac{p \sqrt{2 \omega}}{2}|x|+\tanh ^{-1}\left(\frac{\sqrt{2 \omega}}{|q|}\right)\right)\right]^{-\frac{2}{p}},
$$

desde que $\sqrt{2 \omega}<|q|, \quad\left(0<\omega<\frac{q^{2}}{2}\right), q \neq 0$. Além disso, nas mesmas condições acima com $1<p<\infty, \phi_{\omega}$ em (1.9) é a única solução positiva em $H^{1}(\mathbb{R})$ e $e^{i \omega t} \phi_{\omega}(x)$ é estável em $H^{1}(\mathbb{R})$.

Os nossos principais resultados são os seguintes. Usando a teoria de bifurcação (veja [22, 34]), mostramos que existe uma variedade invariante centro $\mathcal{W}_{\mu}^{p}$, que consiste de órbitas periódicas no tempo da forma $e^{-i(E t-\theta)} \psi_{E}(x)$, com $\psi_{E} \in \mathscr{D}\left(H_{q}\right)$, tal que $\psi_{E}$ é a solução positiva e satisfaz

$$
\left(H_{q}-\lambda\left|\psi_{E}(x)\right|^{p}\right) \psi_{E}(x)=E \psi_{E}(x), \text { para } x \in \mathbb{R}-\{0\} .
$$

Com base nessa existência, estudamos a dinâmica associada à variedade invariante centro

$$
\mathcal{W}_{\mu}^{p}=\left\{e^{i \theta} \psi_{E}(x):\left|E-E_{0}\right|<\mu \text { e } 0 \leq \theta \leq 2 \pi\right\},
$$

pelo fluxo do modelo (1.3). Nosso principal resultado associado a $\mathcal{W}_{\mu}^{p}$ é o seguinte,

Teorema 6.3.1 Considere o problema (1.3). Suponhamos que $\|x u(t)\|_{H^{1}}$ é suficientemente pequena para todo $t \in \mathbb{R}$. Seja $s>1$ e $\frac{1}{2}<\beta \leq 1$. Suponha $u(0) \in L_{s+2 \beta}^{2} \cap H_{1}^{1}(\mathbb{R}) \cap H_{2}^{2}(\Omega), 2(s+2 \beta) \leq p$ e $\|u(0)\|_{L_{s+2 \beta}^{2} \cap H^{1}}$ é suficientemente pequena. Então existem funções diferenciáveis $E(t), \theta(t)$ tal que os limites

$$
\begin{aligned}
& E_{ \pm}=\lim _{t \rightarrow \pm \infty} E(t), \\
& \theta_{ \pm}=\lim _{t \rightarrow \pm \infty} \theta(t),
\end{aligned}
$$

existem e

$$
\lim _{t \rightarrow \pm \infty}\left\|u(t)-e^{-i\left(\int_{0}^{t} E(s) d s-\theta(t)\right)} \psi_{E(t)}\right\|_{L_{-s-2 \beta}^{2}}=0,
$$

onde $u(t)$ é a solução de (3.1) com condição inicial u(0).

A equação (1.11) nos diz que $u$ converge para a órbita periódica no tempo de $e^{i \theta_{ \pm} t} \psi_{E_{ \pm}}$. Note que a parte dispersiva, $u(t)-e^{-i \int_{0}^{t} E(\rho) d \rho} e^{i \theta(t)} \psi_{E(t)}$, converge para zero em $L_{-s-2 \beta}^{2}$. Para provar o Teorema 6.3.1, mostramos algumas propriedades dispersivas do grupo associado ao operador $H_{q}$ 
em espaços de Sobolev com peso e também algumas propriedades da solução no espaço contínuo - $\mathscr{R}\left(P_{c}\right)$; além disso, provamos uma estimativa para o grupo $e^{-i t H_{q}}$ restrito ao espaço contínuo associado ao operador $H_{q}, q<0$, entre os espaços $L_{s+2 \beta}^{2}$ e $L_{-s-2 \beta}^{2}$, isto é, obtemos o seguinte resultado,

Teorema 6.1.1 Para todo $s>1$ e $0 \leq \beta \leq 1$, existe uma constante $C$ independente de $f$ et tal que

$$
\left\|e^{-i t H_{q}} P_{c} f\right\|_{L_{-s-2 \beta}^{2}} \leq C(1+|t|)^{-\frac{1}{2}-\beta}\|f\|_{L_{s+2 \beta}^{2}}, \quad \forall t \in \mathbb{R},
$$

para toda $f \in L_{s+2 \beta}^{2}$.

Na demonstração do Teorema 6.1.1, usamos a fórmula explícita associada ao grupo unitário $e^{-i t H_{q}}$, a saber,

$$
e^{-i t H_{q}}=\frac{1}{2 \pi} \int_{0}^{\infty} e^{-i t \frac{\lambda^{2}}{2}}\left(e_{+}(x, \lambda) \overline{e_{+}(y, \lambda)}+e_{-}(x, \lambda) \overline{e_{-}(y, \lambda)}\right) d \lambda+e^{\frac{1}{2} i t \frac{q^{2}}{2}} P_{p}
$$

$\operatorname{com} P_{p} f=\left\langle f, \psi_{0}\right\rangle f$ e $e_{ \pm}(x, \lambda)=t_{q}(\lambda) e^{ \pm i \lambda x} \chi_{ \pm}^{0}+\left(e^{ \pm i \lambda x}+r_{q}(\lambda) e^{\mp i \lambda x}\right) \chi_{\mp}^{0}$, onde $\chi_{+}^{0}$ é a função característica de $[0,+\infty)$, $\chi_{-}^{0}$ é a função característica de $(-\infty, 0]$ e $t_{q}$ e $r_{q}$ são os coeficientes de transmissão e reflexão, dados por $t_{q}(\lambda)=\frac{i \lambda}{i \lambda-q}$ e $r_{q}(\lambda)=\frac{q}{i \lambda-q}$. Para obter essa fórmula explícita usamos a teoria espectral apresentada por Duchenê et al. em [15] e a Seção 3.2 dessa tese.

Foi necessário estudar o comportamento da solução no espaço contínuo do operador $H_{q}$ para completar a demonstração do Teorema 6.3.1. Estudar as soluções em $\mathscr{R}\left(P_{c}\right)$, só nos garantiu a existência local, embora acreditamos que pode ser provada globalmente. Afim de contornar o problema, dentro das hipóteses do teorema, supomos que $\|x u(t)\|_{H^{1}}$ é suficientemente pequeno para todo $t \in \mathbb{R}$. Então, pensando em não trabalhar com a solução em espaços de Sobolev com peso restrito à $\mathscr{R}\left(P_{c}\right)$, mudamos a não-linearidade do problema (1.3); isto é, nos consideramos também o problema

$$
i \frac{\partial}{\partial t} u=\left(-\frac{1}{2} \Delta+q \delta(x)\right) u+f(x,|u|) \frac{u}{|u|},
$$

onde $f$ é de valor real e satisfaz certas condições sobre regularidade e crescimento como uma função de $u$ e tem decaimento quando $x \rightarrow \pm \infty$. Dentro dessas hipóteses foi possível dizer que o problema (1.12) é bem posto localmente e globalmente aplicando resultados apresentados por Cazenave em [11]. Além disso, acrescentado-se mais uma hipótese de decaimento a $f$, obtemos propriedades assintóticas associadas à variedade invariante centro do problema (1.12).

Descrevemos brevemente os conteúdos de cada capítulo deste trabalho. No Capítulo 2, apresentamos os resultados básicos que serão utilizados no nosso estudo. Na Seção 2.1, estudamos as propriedades associadas ao operador $H_{q}=-\frac{1}{2} \frac{d^{2}}{d x^{2}}+q \delta(x), q \in \mathbb{R}$, visando encontrar as propriedades espectrais associadas ao $H_{q}$ assim como o domínio de $H_{q}, \mathscr{D}\left(H_{q}\right)$. Na Seção 2.2, estudamos algumas propriedades da equação de Schrödinger linear e do grupo $\left\{e^{i t \Delta}\right\}_{t=-\infty}^{t=\infty}$ em $L^{p}(\mathbb{R})$. Na Seção 2.3, estudamos o Teorema de Crandall-Rabinowitz, pois a partir da teoria de bifurcação podemos obter informações sobre as soluções de tipo standing-wave para os modelos (1.3) e (1.12). 
No Capítulo 3, estudamos a boa colocação local e global de $(1.3)$ em $H^{1}(\mathbb{R})$ e apresentamos uma fórmula explícita para o grupo $e^{-i t H_{q}}$ usando a teoria de espalhamento. Finalizamos o capítulo apresentando propriedades do grupo $e^{-i t H_{q}}$ nos espaços $L^{p}$ e $L^{p}$ com peso.

No Capítulo 4, mostramos a existência da variedade centro, onde vemos que é possível aplicar as técnicas da variedade invariante para a classe de EDP's dispersivas. Mostrar a existência da variedade centro será importante para os nossos principais resultados que estão no Capítulo 6 e também para fazer a aplicação que está no Capítulo 7. Nesse capítulo, iniciamos mostrando a existência de uma curva solução para (1.3), aplicando o Teorema de Crandall-Rabinowitz.

No Capítulo 5, fizemos um estudo sobre as soluções de (1.3) em espaço de Sobolev com peso e além disso, apresentamos propriedades do grupo $e^{-i t H_{q}}$ também em espaços de Sobolev com peso.

No Capítulo 6 encontra-se o nosso principal resultado que nos dá a aproximação à variedade centro no espaço $L^{2}$ com peso; ou seja, $L_{-s-2 \beta}^{2}$, com $s>1$ e $\frac{1}{2}<\beta \leq 1$. Antes de mostrá-lo, foi necessário mostrar uma estimativa dispersiva para o grupo $e^{-i t H_{q}}$ sobre o espaço contínuo associado ao operador $H_{q}$ nos espaços de Sobolev com peso e $L_{-s-2 \beta}^{2}$, com $s>1$ e $0 \leq \beta \leq 1$. Tal estimativa também terá importância no Capítulo 7.

No Capítulo 7, onde estudamos o modelo (1.12). As condições impostas sobre a nãolinearidade dadas no Teorema 7.2.1 abaixo, vai induzir uma análise assintótica sem passar por espaço de Sobolev com peso.

Teorema 7.2.1 Suponhamos que para cada $x \in \mathbb{R}, f(x, \cdot) \in C^{1}(\mathbb{R}, \mathbb{R}), \frac{\partial}{\partial x} f(x, \cdot) \in$ $C(\mathbb{R}, \mathbb{R}), f(x, 0)=0$ e, para algum $p>2$,

$$
\left|\frac{\partial}{\partial u} f(x, u)\right| \leq q(x)|u|^{p-1}
$$

onde $(1+|x|)^{2 s+4 \beta} q(x) \in L^{\infty}(\mathbb{R})$, para algum $s>1$ e $1 / 2<\beta \leq 1$. Mais ainda,

$$
\left|\frac{\partial}{\partial x} f(x, u)\right| \leq C|u|^{p} .
$$

Então, existe um $\eta>0$, tal que para todo $u_{0} \in H^{1}(\mathbb{R}) \cap L_{s+2 \beta}^{2}(\mathbb{R})$ com $\left\|u_{0}\right\|_{H^{1}}<\eta$, existem funções, $E(t)$ e $\theta(t)$, em $C^{1}(\mathbb{R}, \mathbb{R})$, tal que para alguma constante $C$ (independe do tempo),

$$
\left\|u(t)-e^{-i \int_{0}^{t} E(\rho) d \rho} e^{i \theta(t)} \psi_{E(t)}\right\|_{L_{-s-2 \beta}^{2}} \leq C\langle t\rangle^{-1 / 2-\beta}\left\|P_{c} u_{0}-h\left(\left\langle u_{0}, \psi_{0}\right\rangle\right)\right\|_{L_{s+2 \beta}^{2}},
$$

onde $u(t)$ é a solução para (7.1) com dado inicial $u_{0}$. Mais ainda, os seguintes limites existem,

$$
\lim _{t \rightarrow \pm \infty} E(t)=E_{ \pm} ; \lim _{t \rightarrow \pm \infty} \theta(t)=\theta_{ \pm} .
$$

Desta forma, será possível obter os principais resultados do Capítulo 6 com menos hipóteses res- 
tritivas. Um modelo muito importante que encaixa-se na equação geral (1.12), é aquela com nãolinearidade não-homogênea

$$
i \frac{\partial u}{\partial t}=H_{q} u+K(x)|u|^{p-1} u, \quad p>1
$$

com $K(x)$ satisfazendo $(1+|x|)^{2 s+4 \beta} K(x) \in L^{\infty}(\mathbb{R})$.

No Capítulo 8 apresentamos nossos planos futuros; isto é, pretendemos estudar a $N L S$ mudando o nosso potencial. Queremos substituir o potencial $\delta$ por $\delta^{\prime}$ (derivada de $\delta$ ) ou soma de duas $\delta$-interação, obtendo os seguintes operadores:

$$
\begin{aligned}
& H_{\alpha}=-\frac{1}{2} \frac{d^{2}}{d x^{2}}+\alpha \delta^{\prime}(x), \\
& H_{\beta}=-\frac{1}{2} \frac{d^{2}}{d x^{2}}+\beta(\delta(x+a)+\delta(x-a)),
\end{aligned}
$$

onde $a \in \mathbb{R}$ e $a>0$.

Enfim, finalizamos com os Apêndices. No Apêndice A, apresentamos como as soluções da $N L S-\delta$ foram construídas e no Apêndice B, colocamos os cálculos que não foram apresentados na demostração do Teorema 6.1.1 e enunciamos o Lema de Schur que foi utilizado em sua demostração. 


\section{Pré-requisitos}

Nesse capítulo começaremos apresentando resultados que nos darão informações sobre as propriedades espectrais do operador

$$
\frac{1}{2} \frac{d^{2}}{d x^{2}}+q \delta
$$

assim como seu domínio. Em seguida, veremos algumas propriedades da equação de Schrödinger linear e também alguma propriedade do grupo $\left\{e^{i t \Delta}\right\}_{t \in \mathbb{R}}$ em $L^{p}\left(\mathbb{R}^{n}\right)$. Enfim, finalizamos um estudo sobre bifurcação, onde a partir do Método de Redução de Lyapunov-Schmidt prova-se o Teorema de Crandall-Rabinowitz. O Teorema de Crandall-Rabinowitz será utilizado no Capítulo 4, onde veremos que o problema

$$
\left(H_{q}-\lambda\left|\psi_{E}\right|^{p}\right) \psi_{E}=E \psi_{E}
$$

possui uma curva $E \mapsto \psi_{E}$ como sua solução que bifurca em $\left(0, E_{0}\right)$, sendo $E_{0}$ o autovalor do operador $H_{q}, q<0$. Tal $\psi_{E}$ é relacionada à solução (1.8), se $0<E<E_{0}$ e $\lambda>0$; à solução (1.9), se $E>E_{0}$ e $\lambda<0$.

\subsection{Extensões autoadjuntas}

Nessa seção, descrevemos algumas propriedades básicas associadas ao operador $H_{q}=$ $-\frac{1}{2} \Delta+q \delta(x)$, para todo $q \in \mathbb{R}$, que nos serão úteis. O que descrevemos a seguir são resultado extraídos de Albeverio et al. [3].

Definição 2.1.1. Seja $A_{0}$ um operador simétrico densamente definido sobre um espaço de Hilbert. Denotaremos por $A_{0}^{*}$ seu adjunto. Consideramos os subespaços

$$
D_{+}=\mathscr{N}\left(A_{0}^{*}-i\right) \quad \text { e } \quad D_{-}=\mathscr{N}\left(A_{0}^{*}+i\right)
$$

$D_{+}$e $D_{-}$são chamados os subespaços de deficiência de $A_{0}$. O par de números $\left(n_{+}\left(A_{0}\right), n_{-}\left(A_{0}\right)\right)$, dados por

$$
n_{+}\left(A_{0}\right)=\operatorname{dim}\left(D_{+}\right) \quad \text { e } n_{-}\left(A_{0}\right)=\operatorname{dim}\left(D_{-}\right),
$$

são chamados os indices de deficiência do operador $A_{0}$.

Agora, consideremos $A=-\frac{d^{2}}{d x^{2}}$ sobre $L^{2}(\mathbb{R})$ com o domínio $\mathscr{D}(A)=H^{2}(\mathbb{R})$ e o operador 
de restrição

$$
\left\{\begin{aligned}
A_{0} & \equiv A \mid \mathscr{D}_{\left(A_{0}\right)}, \\
\mathscr{D}\left(A_{0}\right) & =\{g \in \mathscr{D}(A) \mid g(0)=0\} ;
\end{aligned}\right.
$$

o adjunto de $A_{0}$ é dado por (Ver [3, Seção I.3.1])

$$
\left\{\begin{aligned}
A_{0}^{*} & =-\frac{d^{2}}{d x^{2}} \\
\mathscr{D}\left(A_{0}^{*}\right) & =H^{1}(\mathbb{R}) \cap H^{2}(\mathbb{R}-\{0\}) .
\end{aligned}\right.
$$

Observe que como $\delta=\delta_{0}$ é um funcional linear limitado em $H^{1}(\mathbb{R})$, pois $\left|\left(\delta_{0}, \psi\right)\right|=$ $|\psi(0)|<C\|\psi\|_{H^{1}}$, então $\delta \in H^{-1}(\mathbb{R})$. A demonstração do seguinte Lema pode ser vista seguindo as linha do Lema 3.2 em Angulo \& Ponce [6] e Albeverio et al. [4].

Lema 2.1.1. $O$ operador de restrição $\left.A_{0} \equiv A\right|_{\mathscr{D}\left(A_{0}\right)}$ com

$$
\mathscr{D}\left(A_{0}\right)=\{g \in \mathscr{D}(A) \mid \delta(g)=g(0)=0\}
$$

tem as seguintes propriedades:

(1). Fechado: $\overline{\Gamma\left(A_{0}\right)}=\Gamma\left(A_{0}\right)$, onde $\Gamma\left(A_{0}\right)$ é o gráfico de $A_{0}$;

(2). Simétrico: $\left\langle A_{0} g, h\right\rangle=\left\langle g, A_{0} h\right\rangle$, para todo $g, h \in \mathscr{D}\left(A_{0}\right)$;

(3). Denso: $\overline{\mathscr{D}\left(A_{0}\right)}=L^{2}(\mathbb{R})$;

(4). Os elementos de deficiência de $A_{0}$ são

$$
\left\{\begin{array}{lll}
\text { para } & \lambda=i, & \psi_{+i} \equiv(A-i)^{-1} \delta \\
\text { para } & \lambda=-i, & \psi_{-i} \equiv(A+i)^{-1} \delta
\end{array}\right.
$$

ou seja, $\psi_{ \pm i} \in \mathscr{D}\left(A_{0}^{*}\right)$ e $A_{0}^{*} \psi_{ \pm i}= \pm i \psi_{ \pm i}$. Mais ainda, são $\left(n_{+}\left(A_{0}\right), n_{-}\left(A_{0}\right)\right)=(1,1)$ os índices de deficiência.

A seguir iremos calcular a forma explícita dos elementos de deficiência $\psi_{ \pm i}$ do operador $A_{0}$, os quais serão fundamentais para determinar as suas extensões autoadjuntas. Primeiramente, olhemos para a equação

$$
A_{0}^{*} \psi=k^{2} \psi, \quad \psi \in \mathscr{D}\left(A_{0}^{*}\right), \quad k^{2} \in \mathbb{C}-\mathbb{R}, \quad \operatorname{Im} k>0 .
$$

Note que a solução geral de $A_{0}^{*} \psi=k^{2} \psi$ é

$$
\psi(x)=A e^{-i k x}+B e^{i k x},
$$

logo podemos deduzir que a solução do problema (2.5) com as condições de contorno em (2.5) é dada por

$$
\psi(x)=e^{i k|x|}, \quad \operatorname{Im} k>0 .
$$


Desta maneira, tomando $k^{2}= \pm i, \operatorname{com} \operatorname{Im} \sqrt{ \pm i}>0$, segue que os elementos de deficiência $\psi_{ \pm i}$, com $\left\|\psi_{ \pm i}\right\|=1$, são

$$
\psi_{ \pm i}(x)=\frac{i}{2 \sqrt{ \pm i}} e^{i \sqrt{ \pm i}|x|}, \quad \operatorname{Im} \sqrt{ \pm i}>0
$$

Notamos de $(2.4)$ que $\widehat{\psi_{ \pm i}}(\xi)=\frac{1}{\xi^{2} \pm i}$.

Agora, pela teoria de Von Neumann para extensão de operadores simétricos [36], todas as extensões autoadjuntas $A_{\theta, 0}$ de $A_{0}$ são dadas pela seguinte família a um parâmetro $\theta \in[0,2 \pi)$

$$
\begin{aligned}
\mathscr{D}\left(A_{\theta, 0}\right) & =\left\{g+c \psi_{+i}+c e^{i \theta} \psi_{-i} \mid g \in \mathscr{D}\left(A_{0}\right), c \in \mathbb{C}\right\} \\
A_{\theta, 0}\left(g+c \psi_{+i}+c e^{i \theta} \psi_{-i}\right) & =A_{0} g+i c \psi_{+i}-i c e^{i \theta} \psi_{-i}
\end{aligned}
$$

onde $\psi_{+i}$ e $\psi_{-i}$ são dadas por (2.7).

Para os nossos propósitos, vamos parametrizar as extensões autoadjuntas $A_{\theta, 0}$ com base no parâmetro $q \in \mathbb{R} \cup\{+\infty\}$, ao invés do parâmetro $\theta$ que apareceu nas fórmulas de Von Neumann $(2.8)$ e (2.9). De fato, definindo $\phi(0 \pm)=\lim _{\varepsilon \downarrow 0} \phi(\varepsilon \pm)$ e de (2.7), então para $\xi=g+c \psi_{+i}+c e^{i \theta} \psi_{-i} \in$ $\mathscr{D}\left(A_{\theta, 0}\right)$, temos

$$
\xi^{\prime}(0+)-\xi^{\prime}(0-)=-c\left(1+e^{i \theta}\right)
$$

A seguir, encontramos $q$ tal que $q \xi(0)=-c\left(1+e^{i \theta}\right)$, isto é, $-\left(1+e^{i \theta}\right)=q\left[\psi_{+i}(0)+e^{i \theta} \psi_{-i}(0)\right]$. Com efeito, depois de alguns cálculos encontramos a fórmula

$$
q(\theta)=-\frac{2 \cos \left(\frac{\theta}{2}\right)}{\cos \left(\frac{\theta}{2}-\frac{\pi}{4}\right)}, \quad \theta \in[0,2 \pi) \backslash\left\{\frac{3 \pi}{2}\right\},
$$

pois,

$$
\cos \left(\frac{\theta}{2}-\frac{\pi}{4}\right)=0 \Leftrightarrow \theta=\frac{3 \pi}{2}
$$

Portanto, se $\theta$ varia em $[0,2 \pi), q=q(\theta)$ varia em $\mathbb{R} \cup\{+\infty\}$ e para $\theta_{0}=\frac{3 \pi}{2}$, temos $\lim _{\theta \rightarrow \theta_{0}^{-}} q(\theta)=+\infty$, ou seja, para $\theta=\frac{3 \pi}{2}$ a função $q$ tem uma assíntota vertical que pode ser observado no gráfico a seguir

O teorema abaixo nos dará as extensões autoadjuntas $A_{\theta, 0}$ de $A_{0}$ que dependem do parâmetro $q$.

Teorema 2.1.1. Todas as extensões autoadjuntas $-\Delta_{q}$ de $A_{0}$, onde $-\infty<q \leq \infty$ são dadas por

$$
\left\{\begin{array}{l}
-\Delta_{q}=-\frac{d^{2}}{d x^{2}} \\
\mathscr{D}\left(-\Delta_{q}\right)=\left\{g \in H^{1}(\mathbb{R}) \cap H^{2}(\mathbb{R}-\{0\}) \mid g^{\prime}(0+)-g^{\prime}(0-)=q g(0)\right\} .
\end{array}\right.
$$

Se $q=0$, obtemos o operador de Laplace no espaço $L^{2}(\mathbb{R})$, ou seja,

$$
-\Delta=-\frac{d^{2}}{d x^{2}}, \quad \mathscr{D}(-\Delta)=H^{2}(\mathbb{R})
$$




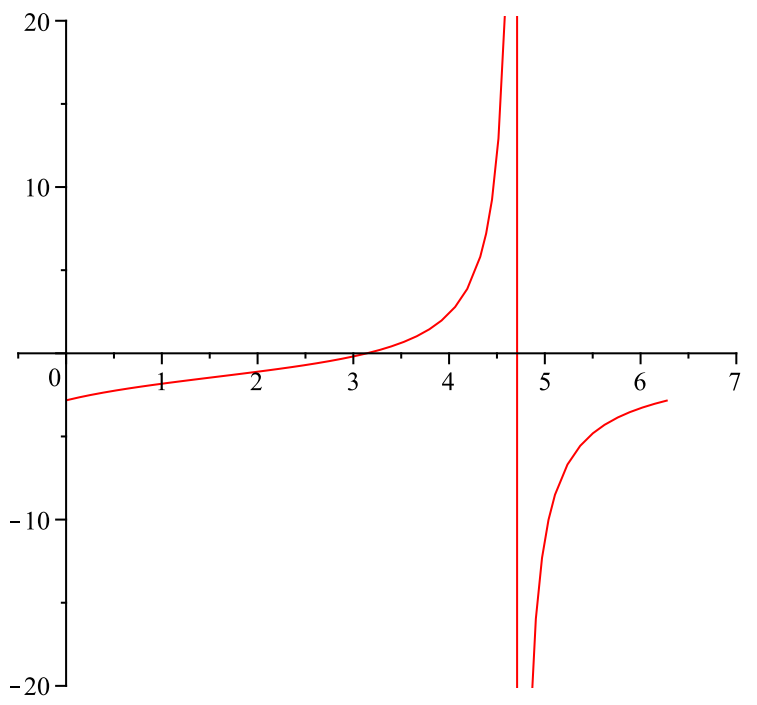

Figura 2.1: Função $q(\theta)$

enquanto se $q=\infty$, a reta real é dividida em dois intervalos $(-\infty, 0)$ e $(0, \infty)$, isto acontece devido a aparição da condição de fronteira do tipo Dirichlet no ponto 0, isto é

$$
\left\{\begin{aligned}
\mathscr{D}\left(-\Delta_{\infty}\right) & =\left\{g \in H^{1}(\mathbb{R}) \cap H^{2}(\mathbb{R}-\{0\}) \mid g(0)=0\right\}=H_{0}^{2}((-\infty, 0)) \oplus H_{0}^{2}((0, \infty)), \\
-\Delta_{\infty} & =\left(-\Delta_{D_{-}}\right) \oplus\left(-\Delta_{D_{+}}\right)
\end{aligned}\right.
$$

onde $\left(-\Delta_{D_{ \pm}}\right)$denota o Laplaciano de Dirichlet sobre $(-\infty, 0),(0, \infty)$, respectivamente, (veja [37], pág. 253), com $\mathscr{D}\left(-\Delta_{D_{-}}\right)=H_{0}^{2}((-\infty, 0))$ e $\mathscr{D}\left(-\Delta_{D_{+}}\right)=H_{0}^{2}((0, \infty))$.

Demonstração: Da equação (2.10), obtemos que $A_{\theta, 0} \subset-\Delta_{q}$, com $q=q(\theta)$ dado pela equação (2.11). Porém, $-\Delta_{q}$ é simétrico sobre seu correspondente domínio $\mathscr{D}\left(-\Delta_{q}\right)$ para todo $-\infty<q \leq \infty$, do qual se obtém que $A_{\theta, 0} \subset-\Delta_{q} \subset\left(-\Delta_{q}\right)^{*} \subset A_{\theta, 0}$, assim finalizamos a demonstração.

Observação 2.1.1. Por definição, $-\Delta_{q}$ descreve a $\delta$-interação de força $q$ centrada em $0 \in \mathbb{R}$. Dito de outra forma, a equação (2.12) é a formulação precisa da expressão formal $-\Delta_{q}=-\frac{d^{2}}{d x^{2}}+q \delta_{0}(x)$; a saber, para $\psi \in \mathscr{D}\left(-\Delta_{q}\right)$, com $x \neq 0,-\Delta_{q} \psi(x)=-\psi^{\prime \prime}(x)$.

A seguir da fórmula de Krein (veja [3, Teorema A.2]), obtemos a seguinte representação do resolvente para $-\Delta_{q}$.

Teorema 2.1.2. O resolvente de $-\Delta_{q}$ é dado por

$$
\begin{gathered}
\left(-\Delta_{q}-k^{2}\right)^{-1}=\left(-\Delta-k^{2}\right)^{-1}-\frac{2 q k}{i q+2 k}\left\langle\cdot, \overline{G_{k}(\cdot)}\right\rangle G_{k}(\cdot) \\
k^{2} \in \rho\left(-\Delta_{q}\right), \quad \operatorname{Im} k>0, \quad-\infty<q \leq \infty
\end{gathered}
$$

onde

$$
G_{k}(x)=\frac{i}{2 k} e^{i k|x|}, \quad \operatorname{Im} k>0,
$$


em outras palavras,

$$
\left(-\Delta_{q}-k^{2}\right)^{-1} f(\xi)=\int \mathbb{K}(\xi-y) f(y) d y,
$$

com núcleo integral $\mathbb{K}$ expressado como

$$
\begin{gathered}
\mathbb{K}(u-v)=\frac{i}{2 k} e^{i k|u-v|}+\frac{q}{2 k(i q+2 k)} e^{i k[|u|+|v|]} \\
k^{2} \in \rho\left(-\Delta_{q}\right), \quad \operatorname{Im} k>0, \quad u, v \in \mathbb{R} .
\end{gathered}
$$

Demonstração: Seja $f \in L^{2}(\mathbb{R}), \operatorname{Im} k>0, k \neq-\frac{i q}{2}$ e definamos

$$
h_{q}(x)=\left(\left(-\Delta-k^{2}\right)^{-1} f\right)(x)-\frac{2 q k}{i q+2 k}\left\langle f, \overline{G_{k}(x)}\right\rangle G_{k}(x) .
$$

Observe que $h_{q} \in H^{1}(\mathbb{R}) \cap H^{2}(\mathbb{R}-\{0\})$. Como $G_{k}^{\prime}(0+)-G_{k}^{\prime}(0-)=-1$, derivando $h_{q}$ obtemos

$$
h_{q}^{\prime}(0+)-h_{q}^{\prime}(0-)=\frac{i q}{i q+2 k} \int_{\mathbb{R}} e^{i k\left|x^{\prime}\right|} f\left(x^{\prime}\right) d x^{\prime}=q h_{q}(0),
$$

$\operatorname{assim} h_{q} \in \mathscr{D}\left(-\Delta_{q}\right)$. Mais ainda, para $x \in \mathbb{R}-\{0\},-G_{k}^{\prime \prime}(x)-k^{2} G_{k}(x)=0$, portanto, pelo Teorema 2.1.1 e Observação 2.1.1, para $x \neq 0$,

$$
\left(\left(-\Delta_{q}-k^{2}\right) h_{q}\right)(x)=-h_{q}^{\prime \prime}(x)-k^{2} h_{q}(x)=f(x) .
$$

Note que da equação (2.18)

$$
h_{q}(x)=\int_{\mathbb{R}}\left[\frac{i}{2 k} e^{i k|x-y|}+\frac{q}{2 k(i q+2 k)} e^{i k(|y|+|x|)}\right] f(y) d y=\int \mathbb{K}(x-y) f(y) d y,
$$

mostrando assim a equação (2.17).

O seguinte resultado dá uma caracterização dos elementos do $\mathscr{D}\left(-\Delta_{q}\right)$.

Teorema 2.1.3. O dominio $\mathscr{D}\left(-\Delta_{q}\right),-\infty<q \leq \infty$, consiste de todos os elementos $\psi$ do tipo

$$
\psi(x)=\phi_{k}(x)-\frac{2 q k}{i q+2 k} \phi_{k}(0) G_{k}(x)
$$

onde $\phi_{k} \in \mathscr{D}(-\Delta)=H^{2}(\mathbb{R})$ e $k^{2} \in \rho\left(-\Delta_{q}\right)$, $\operatorname{Im} k>0$. A decomposição (2.21) é única com $\psi \in \mathscr{D}\left(-\Delta_{q}\right)$, desta forma, obtemos

$$
\left(-\Delta_{q}-k^{2}\right) \psi=\left(-\Delta-k^{2}\right) \phi_{k} .
$$

Mais ainda, suponhamos que $\psi \in \mathscr{D}\left(-\Delta_{q}\right)$ e que $\psi=0$ em um aberto $U \subseteq \mathbb{R}$, então $-\Delta_{q} \psi=0$ em $U$.

Demonstração: Veja [3, Teorema 3.1.3]. 
Quanto as propriedades espectral, para $\sigma_{e s s}(A)$ e $\sigma_{p}(A)$ representando o espectro essencial e o espectro pontual do operador $A$, respectivamente, temos o seguinte teorema.

Teorema 2.1.4. Seja $-\infty<q \leq \infty$. O espectro essencial de $-\Delta_{q}=-\frac{d^{2}}{d x^{2}}+q \delta$ é o eixo real não negativo, $\sigma_{\text {ess }}\left(-\Delta_{q}\right)=[0, \infty)$. Se $-\infty<q<0,-\Delta_{q}$ tem precisamente um autovalor simples $e$ negativo, isto é, $\sigma_{p}\left(-\Delta_{q}\right)=\left\{-\frac{q^{2}}{4}\right\}$, com $\psi_{q}(x)=\sqrt{-\frac{q}{2}} e^{\frac{1}{2} q|x|}$ sendo sua autofunção normalizada e estritamente positiva. Se $q \geq 0$ ou $q=\infty,-\Delta_{q}$ não tem autovalores, $\sigma_{p}\left(-\Delta_{q}\right)=\emptyset$.

Demonstração: Segue da fórmula (2.15), pois os autovalores discretos correspondem aos pólos do resolvente na variável $k^{2}$, ou seja, $k^{2}=-\frac{q^{2}}{4}$.

Finalmente dos Teoremas 2.1.1 e 2.1.4, temos o seguinte resumo para $q<0$ :

$$
H_{q} \equiv-\frac{1}{2} \Delta+q \delta_{0}(x)=\frac{1}{2}\left(-\Delta+2 q \delta_{0}(x)\right)
$$

tem um único autovalor negativo, $\sigma_{p}\left(H_{q}\right)=\left\{-\frac{q^{2}}{2}\right\}$ com autofunção normalizada $\sqrt{-q} e^{q|x|}$; além disso, $\mathscr{D}\left(H_{q}\right)=\left\{u \in H^{1}(\mathbb{R}) \cap H^{2}(\mathbb{R}-\{0\}) \mid u^{\prime}(0+)-u^{\prime}(0-)=2 q u(0)\right\}$.

A partir deste momento, denotaremos o autovalor e autofunção do operador $H_{q}$, para $q<0$,

$$
\begin{aligned}
E_{0} & =-\frac{q^{2}}{2} \\
\psi_{0}(x) & =\sqrt{-q} e^{q|x|},
\end{aligned}
$$

respectivamente.

Observação 2.1.2. Note que $\psi_{0} \in H^{1}(\mathbb{R})$, porém $\psi_{0} \notin H^{s}(\mathbb{R}), s>1$. Por outro lado, $\psi_{0} \in$ $H^{s}(\mathbb{R}-\{0\})$, para todo $s \geq 1$.

\subsection{Equação de Schrödinger linear}

Posteriormente veremos que algumas propriedades da equação de Schrödinger linear também ocorrem na NLS- $\delta$ linear. Assim, vamos inicialmente estabelecer algumas propriedades bem conhecidas do problema de valor inicial para a equação de Schrödinger linear

$$
\left\{\begin{array}{l}
\frac{\partial u}{\partial t}=i \Delta u \\
u(x, 0)=u_{0}(x),
\end{array}\right.
$$


onde $x \in \mathbb{R}^{n}$ e $t \in \mathbb{R}$. Usando a transformada de Fourier, temos que a solução $u=u(x, t)$ de $(2.25)$ vem dada por,

$$
\begin{aligned}
u(x, t) & =\left(e^{-4 \pi^{2} i t|y|^{2}} \hat{u}_{0}(y)\right)^{\vee}(x)=\frac{e^{-\frac{|x|^{2}}{4 i t}}}{(4 \pi i t)^{\frac{n}{2}}} * u_{0}(x) \\
& =\left(\frac{1}{4 \pi i t}\right)^{\frac{n}{2}} \int_{\mathbb{R}^{n}} e^{-\frac{|x-y|^{2}}{4 i t}} u_{0}(y) d y,
\end{aligned}
$$

onde * denota a convolução, " " a Transformada de Fourier e "V" a Transformada de Fourier Inversa. Iremos denotar a solução de (2.25), como $u(x, t)=e^{i t \Delta} u_{0}(x)$.

Os resultados que apresentamos a seguir, podem ser encontrados em [31].

Na seguinte proposição, listaremos as simetrias básicas de (2.25) obtidas através da invariância da equação.

Proposição 2.1. Se $u=u(x, t)$ é uma solução de (2.25), então para

1. $u_{1}(x, t)=e^{i \theta} u(x, t), \theta \in \mathbb{R}$ fixo;

2. $u_{2}(x, t)=u\left(x-x_{0}, t-t_{0}\right)$, com $x_{0} \in \mathbb{R}^{n}, t_{0} \in \mathbb{R}$ fixos;

3. $u_{3}(x, t)=u(A x, t)$, com A qualquer matriz ortogonal $n \times n$;

4. $u_{4}(x, t)=u\left(x-2 x_{0} t, t\right) e^{i\left(x \cdot x_{0}-\left|x_{0}\right|^{2} t\right)}, \operatorname{com} x_{0} \in \mathbb{R}^{n}$ fixo;

5. $u_{5}(x, t)=\lambda^{n / 2} u\left(\lambda x, \lambda^{2} t\right), \lambda \in \mathbb{R}$ fixo;

temos que $u_{i}, 1 \leq i \leq 5$, também satisfaz a equação (2.25).

A seguir estabeleceremos que a família de operadores $\left\{e^{i t \Delta}\right\}_{t=-\infty}^{-\infty}$ forma um grupo unitário sobre o espaço de Hilbert $L^{2}\left(\mathbb{R}^{n}\right)$.

Proposição 2.2. 1. $e^{i 0 \Delta}=I$

2. $e^{i t \Delta} e^{i t^{\prime} \Delta}=e^{i\left(t+t^{\prime}\right) \Delta}, \operatorname{com}\left(e^{i t \Delta}\right)^{-1}=e^{-i t \Delta}=\overline{e^{i t \Delta}}$.

3. Para todo $t \in \mathbb{R}, e^{i t \Delta}: L^{2}\left(\mathbb{R}^{n}\right) \rightarrow L^{2}\left(\mathbb{R}^{n}\right)$ é uma isometria; a qual implica que

$$
\left\|e^{i t \Delta} f\right\|_{2}=\|f\|_{2}
$$

4. Fixando $f \in L^{2}\left(\mathbb{R}^{n}\right)$, a função $\Phi_{f}: \mathbb{R} \rightarrow L^{2}\left(\mathbb{R}^{n}\right)$ dada por $\Phi_{f}(t)=e^{i t \Delta} f$ é uma função contínua; isto é, descreve uma curva em $L^{2}\left(\mathbb{R}^{n}\right)$.

Demonstração: Segue-se imediatamente das propriedades da Transformada de Fourier, do Teorema de Plancherel e do Teorema da Convergência Dominada de Lebesgue.

Agora veremos uma propriedade muito importante do grupo $\left\{e^{i t \Delta}\right\}_{t=-\infty}^{+\infty}$ em $L^{p}\left(\mathbb{R}^{n}\right)$. 
Lema 2.2.1. Se $t \neq 0, \frac{1}{p}+\frac{1}{p^{\prime}}=1$ e $p \in[1,2]$, então $e^{i t \Delta}: L^{p}\left(\mathbb{R}^{n}\right) \rightarrow L^{p^{\prime}}\left(\mathbb{R}^{n}\right)$ é contínua e

$$
\left\|e^{i t \Delta} f\right\|_{p^{\prime}} \leq C|t|^{-n\left(\frac{1}{p}-\frac{1}{2}\right)}\|f\|_{p}
$$

Demonstração: Primeiramente, seja $p=2, \operatorname{logo} p^{\prime}=2$. Usando o item 3 da Proposição 2.2, obtemos $\left\|e^{i t \Delta} f\right\|_{2}=\|f\|_{2}$. Agora, consideremos $p=1$, assim $p^{\prime}=\infty$, logo de (2.26) e a desigualdade de Young, segue-se que

$$
\left\|e^{i t \Delta} f\right\|_{\infty} \leq\left\|\left(\frac{1}{4 \pi i t}\right)^{\frac{n}{2}} e^{-\frac{|x|^{2}}{4 i t}}\right\|_{\infty}\|f\|_{1}=\left(\frac{1}{4 \pi}\right)^{\frac{n}{2}}|t|^{-\frac{n}{2}}\left\|e^{-\frac{|x|^{2}}{4 i t}}\right\|_{\infty}\|f\|_{1}=C|t|^{-\frac{n}{2}}\|f\|_{1} .
$$

Assim, temos que $e^{i t \Delta}: L^{2} \rightarrow L^{2}$ e $e^{i t \Delta}: L^{1} \rightarrow L^{\infty}$ são operadores lineares limitados e portanto, pelo Teorema de Riesz-Thorim obtemos $e^{i t \Delta}: L^{p} \rightarrow L^{p^{\prime}}$ com

$$
\frac{1}{p}=\frac{\theta}{2}+\frac{1-\theta}{1}, \frac{1}{p^{\prime}}=\frac{\theta}{2}+\frac{1-\theta}{\infty}=\frac{\theta}{2}, \theta \in(0,1)
$$

$\log \mathrm{O}$

$$
\frac{1}{p}+\frac{1}{p^{\prime}}=1 \quad \text { e } \quad \frac{1}{p}-\frac{1}{p^{\prime}}=1-\theta=1-\frac{2}{p^{\prime}} .
$$

Portanto,

$$
\left\|e^{i t \Delta} f\right\|_{p^{\prime}} \leq\left(C|t|^{-\frac{n}{2}}\right)^{1-\theta}\|f\|_{p}=C|t|^{-\frac{n}{2}\left(\frac{1}{p}-\frac{1}{p^{\prime}}\right)}\|f\|_{p}=C|t|^{-n\left(\frac{1}{p}-\frac{1}{2}\right)}\|f\|_{p}
$$

\subsection{Bifurcação com o núcleo unidimensional}

Nesta seção, vamos apresentar teoremas e definições que foram extraídos de [22]. Poderíamos deixar a referência, mas optamos por apresentá-las, pois deixamos algumas passagens nas demonstrações dos teoremas mais claras. Além disso, as provas vão contribuir para o desencadear dos resultados que apresentaremos nessa tese. Finalizaremos essa seção com um exemplo, onde aplicamos a teoria da bifurcação.

Suponhamos que exista uma curva solução de $F(x, \kappa)=0$ através de $\left(x_{0}, \kappa_{0}\right)$, onde $x \in X$, sendo $X$ um espaço de Banach, e $\kappa \in \mathbb{R}$. Mostraremos a existência de uma segunda curva solução em $\left(x_{0}, \kappa_{0}\right)$; ou seja, o que chamamos de bifurcação. Para que isto aconteça, assumiremos ainda que $\frac{\partial F}{\partial x}\left(x_{0}, \kappa_{0}\right)$ não é bijetora, para podermos excluir a aplicação do Teorema da Função Implícita próximo de $\left(x_{0}, \kappa_{0}\right)$.

Normalizamos a primeira curva de soluções para a chamada "solução trivial" $\{(0, \kappa) \mid \kappa \in \mathbb{R}\}$. A saber, se $F(x(s), \kappa(s))=0$, então consideramos $\hat{F}(x, s)=F(x(s)+x, \kappa(s))$, e obviamente, $\hat{F}(0, s)=F(x(s), \kappa(s))=0$, para todo parâmetro $s$. 
Antes de prosseguirmos, consideremos a seguinte definição:

Definição 2.3.1. Sejam $X$ e $Z$ espaços de Banach. A aplicação contínua $\mathcal{F}: U \longrightarrow Z$, onde $U \subset X$ é um aberto, é um operador de Fredholm não-linear se, para todo $x \in U, \mathcal{F}$ é Frechet diferenciável em $x$ e sua diferencial, $D \mathcal{F}(x)^{*}$, satisfaz os seguintes itens:

(i) $\operatorname{dim} \mathscr{N}(D \mathcal{F}(x))<\infty$;

(ii) $\operatorname{codim} \mathscr{R}(D \mathcal{F}(x))<\infty$;

(iii) $\mathscr{R}(D \mathcal{F}(x))$ é um subespaço linear fechado de $Z$.

O inteiro $\operatorname{dim} \mathscr{N}(D \mathcal{F}(x))-\operatorname{codim} \mathscr{R}(D \mathcal{F}(x))$ é chamado indice de Fredholm

Em geral, vamos estudar aplicações na forma $F: X \times \mathbb{R} \longrightarrow Z$, onde $X$ e $Z$ são espaços de Banach. Porém, para ter um conhecimento mais geral, vamos começar com a aplicação $F$ : $X \times Y \longrightarrow Z$, sendo $X, Y$ e $Z$ espaços de Banach. Assim, sejam $U \subset X$ e $V \subset Y$ abertos. Consideremos $F: U \times V \longrightarrow Z$ tal que

- $F\left(x_{0}, y_{0}\right)=0$, para algum $\left(x_{0}, y_{0}\right) \in U \times V$;

- $F \in C(U \times V, Z)$;

- $\frac{\partial F}{\partial x} \in C(U \times V, \mathcal{L}(X, Z))$.

Agora vamos supor que em $y=y_{0}, F$ é um operador de Fredholm não-linear com relação a $x$; dito de outra forma, $F\left(\cdot, y_{0}\right): U \longrightarrow Z$ satisfaz a Definição 2.3.1. Portanto, sabe-se que $N=\mathscr{N}\left(\frac{\partial F}{\partial x}\left(x_{0}, y_{0}\right)\right)$ e $Z_{0}=\left(\mathscr{R}\left(\frac{\partial F}{\partial x}\left(x_{0}, y_{0}\right)\right)\right)^{\perp}$ são espaços de dimensão finita. Então, existem complementos fechados, $X_{0}$ e $Z_{0}$ em $X$ e $Z$, para $\left(\frac{\partial F}{\partial x}\left(x_{0}, y_{0}\right)\right)$ e $\mathscr{R}\left(\frac{\partial F}{\partial x}\left(x_{0}, y_{0}\right)\right)$, respectivamente, tais que

$$
\begin{aligned}
X & =\mathscr{N}\left(\frac{\partial F}{\partial x}\left(x_{0}, y_{0}\right)\right) \oplus X_{0}, \\
Z & =\mathscr{R}\left(\frac{\partial F}{\partial x}\left(x_{0}, y_{0}\right)\right) \oplus Z_{0} .
\end{aligned}
$$

Desta forma, tais decomposições definem as seguintes projeções ortogonais

$$
\begin{aligned}
& P: X \longrightarrow N, P^{2}=P, P=P^{*}, \\
& Q: Z \longrightarrow Z_{0}, Q^{2}=Q, Q=Q^{*},
\end{aligned}
$$

ao longo de $X_{0}$ e $\mathscr{R}\left(\frac{\partial F}{\partial x}\left(x_{0}, y_{0}\right)\right)$, respectivamente, a saber, para $x=n+m \in X, P(x)=n$ e para $y=r+s \in Z, Q(y)=r$.

O teorema abaixo nos mostra que sob as condições acima o Método de Redução de Lyapunov-Schmidt pode ser aplicado.

\footnotetext{
* Jacobiana de $\mathcal{F}$ em $x$
} 
Teorema 2.3.1. Com as hipóteses acima sobre $F$ temos que existe uma vizinhança $U_{2} \times V_{2}$ de $\left(x_{0}, y_{0}\right)$ em $U \times V \subset X \times Y$ tal que o problema

$$
F(x, y)=0,(x, y) \in U_{2} \times V_{2}
$$

é equivalente a considerar o problema (um problema de dimensão finita) para $x=v+w$

$$
\Phi(v, y)=Q F(v+\psi(v, y))=0,(v, y) \in \tilde{U} \times \tilde{V} \subset N \times Y
$$

onde $\Phi$ satisfaz: $\Phi \in C\left(\tilde{U} \times \tilde{V}, Z_{0}\right), \Phi\left(v_{0}, y_{0}\right)=0,\left(v_{0}, y_{0}\right) \in \tilde{U} \times \tilde{V}$, com $x_{0}=P x_{0}+(I-P) x_{0} \equiv$ $v_{0}+w_{0}$ e $\psi$ sendo uma função contínua específica. A função $\Phi$ é chamada a função de bifurcação associada ao problema (2.29).

Demonstração: Definamos $P x=v \in N$ e $(I-P) x=w \in X_{0}$. Então, de (2.29), obtemos

$$
Q F(P x+(I-P) x, y)+(I-Q) F(P x+(I-P) x, y)=0
$$

logo temos o seguinte sistema equivalente

$$
\begin{aligned}
Q F(v+w, y)=Q F(P x+(I-P) x, y) & =0 \\
(I-Q) F(v+w, y)=(I-Q) F(P x+(I-P) x, y) & =0 .
\end{aligned}
$$

A seguir, consideremos a aplicação

$$
\begin{aligned}
G: \tilde{U}_{2} \times W_{2} \times V_{2} \subseteq N \times X_{0} \times V_{2} & \longrightarrow \mathscr{R}\left(\frac{\partial F}{\partial x}\left(x_{0}, y_{0}\right)\right) \\
(v, w, y) & \longmapsto G(v, w, y)=(I-Q) F(v+w, y) .
\end{aligned}
$$

Note que $\left(v_{0}, w_{0}\right)=\left(P x_{0},(I-P) x_{0}\right) \in \tilde{U}_{2} \times W_{2} \subset N \times X_{0}$, com $\tilde{U}_{2}$ e $W_{2}$ vizinhanças em $N$ e $X_{0}$, respectivamente, tais que $\tilde{U}_{2} \times W_{2} \subset U \subset X$. Agora, notamos que

- $G\left(v_{0}, w_{0}, y_{0}\right)=(I-Q) F\left(x_{0}, y_{0}\right)=0$,

- $\frac{\partial G}{\partial w}\left(v_{0}, w_{0}, y_{0}\right)=(I-Q) \frac{\partial F}{\partial x}\left(x_{0}, y_{0}\right): X_{0} \longrightarrow \mathscr{R}\left(\frac{\partial F}{\partial x}\left(x_{0}, y_{0}\right)\right)$ é bijetora.

De fato, primeiramente vejamos que é injetora. Seja $v \in X_{0}$, tal que

$$
\frac{\partial G}{\partial w}\left(v_{0}, w_{0}, y_{0}\right) v=(I-Q) \frac{\partial F}{\partial x}\left(x_{0}, y_{0}\right) v=0 \Rightarrow \frac{\partial F}{\partial x}\left(x_{0}, y_{0}\right) v=0 \Rightarrow v \in N
$$

$\operatorname{assim} v \in N \cap X_{0}=\{0\}$, isto é, $v=0$. Logo, $\mathscr{N}\left(\frac{\partial G}{\partial w}\left(v_{0}, w_{0}, y_{0}\right)\right)=\{0\}$. Agora, para ver que é sobrejetora, seja $h \in \mathscr{R}\left(\frac{\partial F}{\partial x}\left(x_{0}, y_{0}\right)\right)$, então existe $x \in X$ tal que $\frac{\partial F}{\partial x}\left(x_{0}, y_{0}\right) x=h$. Portanto, considerando a decomposição $x=v+w \in N \oplus X_{0}$, obtemos que

$$
\frac{\partial G}{\partial w}\left(v_{0}, w_{0}, y_{0}\right) w=(I-Q) \frac{\partial F}{\partial x}\left(x_{0}, y_{0}\right) x-(I-Q) \underbrace{\frac{\partial F}{\partial x}\left(x_{0}, y_{0}\right) v}_{=0}=h .
$$


Dessa forma, $\frac{\partial G}{\partial w}\left(v_{0}, w_{0}, y_{0}\right)$ é sobrejetora.

Assim, pelo Teorema da Função Implícita, temos existem um aberto $\tilde{U} \times \tilde{W} \times \tilde{V} \subset \tilde{U}_{2} \times W_{2} \times V_{2} \subset$ $U \times V$ tal que $\left(v_{0}, w_{0}, y_{0}\right) \in \tilde{U} \times \tilde{W} \times \tilde{V}$ e uma única aplicação $\psi: \tilde{U} \times \tilde{V} \longrightarrow \tilde{W} \subset X_{0}$ tal que $\psi \in C(\tilde{U} \times \tilde{V}, \tilde{W}), w_{0}=\psi\left(x_{0}, y_{0}\right)$ e $G(v, w, y)=G(v, \psi(v, y), y)=0$, para cada $(v, y) \in \tilde{U} \times \tilde{V}$ com $w=\psi(v, y)$.

Finalmente, usando $\psi$ podemos definir a função $\Phi(v, y)=Q F(v+\psi(v, y), y)$, para $(v, y) \in$ $\tilde{U} \times \tilde{V}$; logo, pelo visto anteriormente, $\Phi(v, y)=0$. Assim, $F(v+\psi(v, y), y)=0$, pois $(I-Q) F(v+$ $\psi(v, y), y)=0$.

Corolário 2.3.1.1. Seguindo as mesmas notações do Teorema 2.3.1 e supondo que $F \in C^{1}(U \times$ $V, Z)$, obtemos

- $\psi \in C^{1}\left(\tilde{U} \times \tilde{V}, X_{0}\right), \Phi \in C^{1}\left(\tilde{U} \times \tilde{V}, Z_{0}\right) ;$

- para $w_{0}=\psi\left(v_{0}, y_{0}\right)$, temos $\frac{\partial \psi}{\partial v}\left(v_{0}, y_{0}\right) f \equiv 0$, para todo $f \in N$;

- $\frac{\partial \Phi}{\partial v}\left(v_{0}, y_{0}\right) f \equiv 0$, para todo $f \in N$.

Demonstração: Pelo Teorema da Função Implícita, para todo $(v, y) \in \tilde{U} \times \tilde{V} \subset N \times Y$,

$$
\frac{\partial}{\partial v}(G(v, \psi(v, y), y))=(I-Q) \frac{\partial F}{\partial x}(v+\psi(v, y), y)\left(I_{N}+\frac{\partial \psi}{\partial v}(v, y)\right)=0
$$

sendo $I_{N}$ a identidade em $N=\mathscr{N}\left(\frac{\partial F}{\partial x}\left(x_{0}, y_{0}\right)\right)$. Avaliando a igualdade acima em $\left(v_{0}, y_{0}\right)$, temos de $v_{0}+w_{0}=x_{0}$ que essa implica que para toda $f \in N$,

$$
(I-Q) \frac{\partial F}{\partial x}\left(x_{0}, y_{0}\right) \frac{\partial \psi}{\partial v}\left(v_{0}, y_{0}\right) f=0 \Rightarrow \frac{\partial F}{\partial x}\left(x_{0}, y_{0}\right) \frac{\partial \psi}{\partial v}\left(v_{0}, y_{0}\right) f=0 \Rightarrow \frac{\partial \psi}{\partial v}\left(v_{0}, y_{0}\right) f \in N \cap X_{0}
$$

$\operatorname{assim} \frac{\partial \psi}{\partial v}\left(v_{0}, y_{0}\right) \equiv 0$. Finalmente, pela equação $\Phi(v, y)=0$, teremos

$$
\frac{\partial \Phi}{\partial v}(v, y)=Q \frac{\partial F}{\partial x}(v+\psi(v, y), y)\left(I_{N}+\frac{\partial \psi}{\partial v}(v, y)\right)=0
$$

assim, da análise acime e da definição de $Q$, temos em $\left(v_{0}, y_{0}\right)$ e para toda $f \in N$,

$$
\frac{\partial \Phi}{\partial v}\left(v_{0}, y_{0}\right) f=Q \frac{\partial F}{\partial x}\left(x_{0}, y_{0}\right)\left(I_{N}+\frac{\partial \psi}{\partial v}\left(v_{0}, y_{0}\right)\right) f=0
$$

Como consequência do anterior, temos o seguinte importante resultado que será útil em nosso estudo, isto é, o um caso específico do Teorema de Crandall-Rabinowitz quando a dimensão do núcleo é zero. 
Teorema 2.3.2 (Teorema de Crandall-Rabinowitz). Sejam $X, Z$ espaços de Banach, $F: X \times \mathbb{R} \longrightarrow$ $Z$ uma aplicação, onde $U \subset X$ é aberto, $\tilde{v}_{0} \in X$ tal que $\left\|\tilde{v}_{0}\right\|=1$, e $\kappa_{0} \in V \subset \mathbb{R}$ aberto com $V$ aberto. Suponhamos que

$$
\begin{aligned}
& F \in C^{2}(U \times V), \\
& F(0, \kappa)=0, \forall \kappa \in \mathbb{R}, \\
& \operatorname{dim} \mathscr{N}\left(\frac{\partial F}{\partial x}\left(0, \kappa_{0}\right)\right)=\operatorname{codim} \mathscr{R}\left(\frac{\partial F}{\partial x}\left(0, \kappa_{0}\right)\right)=1, \\
& \mathscr{N}\left(\frac{\partial F}{\partial x}\left(0, \kappa_{0}\right)\right)=\left[\tilde{v}_{0}\right], \\
& \frac{\partial^{2} F}{\partial x \partial \kappa}\left(0, \kappa_{0}\right) \tilde{v}_{0} \notin \mathscr{R}\left(\frac{\partial F}{\partial x}\left(0, \kappa_{0}\right)\right) .
\end{aligned}
$$

Então existe uma curva continuamente diferenciável não-trivial passando por $\left(0, \kappa_{0}\right)$ dada por

$$
s \in(-\delta, \delta) \mapsto(x(s), \kappa(s)),
$$

tal que $(x(0), \kappa(0))=\left(0, \kappa_{0}\right), F(x(s), \kappa(s))=0, s \in(-\delta, \delta)$, e todas as soluções de $F(x, \kappa)=0$ em uma vizinhança de $\left(0, \kappa_{0}\right)$ estão sobre a solução trivial $\kappa \mapsto(0, \kappa)$ ou sobre a curva não-trivial (2.38). A interseção $\left(0, \kappa_{0}\right)$ destas duas curvas é chamado um ponto de bifurcação.

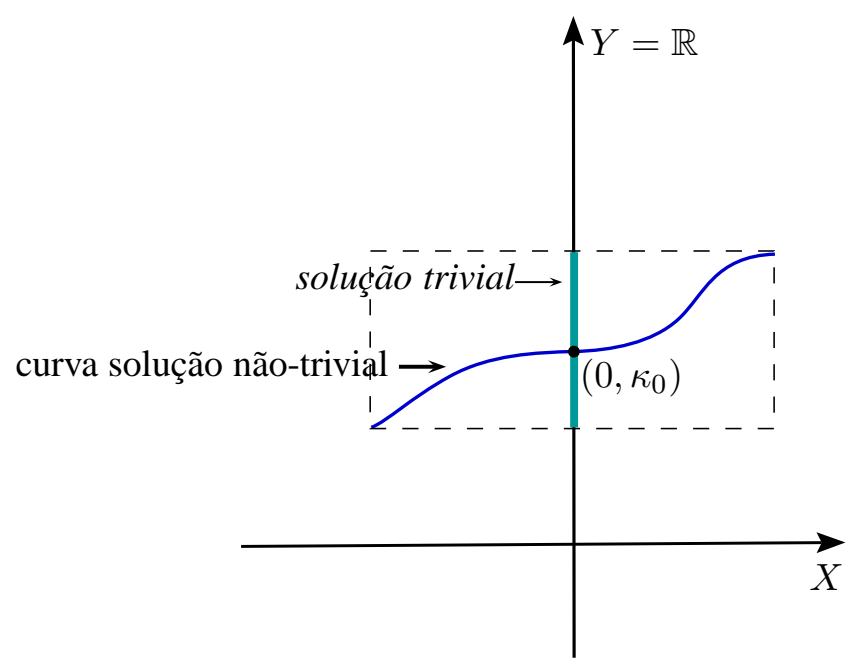

Figura 2.2: Diagrama de Bifurcação

Demonstração: Pelo Teorema 2.3.1, o problema $F(x, \kappa)=0$ para $(x, \kappa)$ próximo de $\left(0, \kappa_{0}\right)$ é equivalente a chamada equação de bifurcação; ou seja, para $\operatorname{dim} Z_{0}=\operatorname{codim} \mathscr{R}\left(\frac{\partial F}{\partial x}\left(0, \kappa_{0}\right)\right)=1$,

$$
\begin{aligned}
& \Phi: \tilde{U} \times \tilde{V} \longrightarrow Z_{0}, \\
& \Phi(v, \kappa)=Q F(v+\psi(v, \kappa), \kappa)=0,
\end{aligned}
$$

para $(v, \kappa)$ próximo de $\left(0, \kappa_{0}\right),(v, \kappa) \in \tilde{U} \times \tilde{V} \subset N \times \mathbb{R}, \Phi \in C^{2}\left(\tilde{U} \times \tilde{V}, Z_{0}\right)$ e $\Phi\left(0, \kappa_{0}\right)=0$. Agora, por (2.34) e pela prova do Teorema 2.3.1, $G(0,0, \kappa)=(I-Q) F(0, \kappa)=0$ e do Corolário 2.3.1.1 
teremos

$$
\psi(0, \kappa)=0, \forall \kappa \in \tilde{V}
$$

Logo, inserindo $(v, \kappa)=(0, \kappa)$ na função bifurcação teremos

$$
\Phi(0, \kappa)=Q F(0+\psi(0, \kappa), \kappa)=Q F(0, \kappa)=0, \forall \kappa \in \tilde{V} \subset \mathbb{R}
$$

e assim obtemos a solução trivial. Notamos que do Corolário 2.3.1.1 e (2.34),

$$
\begin{aligned}
& \frac{\partial \Phi}{\partial v}\left(0, \kappa_{0}\right)=0 \\
& \frac{\partial \Phi}{\partial \kappa}\left(0, \kappa_{0}\right)=Q \frac{\partial F}{\partial x}\left(0, \kappa_{0}\right) \frac{\partial \psi}{\partial \kappa}\left(0, \kappa_{0}\right)+Q \frac{\partial F}{\partial \kappa}\left(0, \kappa_{0}\right)=Q \frac{\partial F}{\partial \kappa}\left(0, \kappa_{0}\right)=0
\end{aligned}
$$

assim, não podemos aplicar o Teorema da Função Implícita imediatamente. Desta forma, observe inicialmente

$$
\Phi(v, \kappa)=\Phi(v, \kappa)-\Phi(0, \kappa)=\int_{0}^{1} \frac{d \Phi}{d t}(t v, \kappa) d t=\int_{0}^{1} \frac{\partial \Phi}{\partial v}(t v, \kappa) v d t
$$

$\operatorname{para}(v, \kappa) \in \tilde{U} \times \tilde{V} \subset N \times \mathbb{R}, N=\mathscr{N}\left(\frac{\partial F}{\partial x}\left(0, \kappa_{0}\right)\right)=\left[\tilde{v}_{0}\right]$. Assim, definindo $v=s \tilde{v}_{0}, s \in(-\delta, \delta)$, para $v \in \tilde{U} \subset N$, consideremos a função

$$
\begin{aligned}
\tilde{\Phi}:(-\delta, \delta) \times \tilde{V} & \longrightarrow \mathbb{R} \approx Z_{0} \\
(s, \kappa) & \longmapsto \tilde{\Phi}(s, \kappa)=\frac{\Phi\left(s \tilde{v}_{0}, \kappa\right)}{s}=\int_{0}^{1} \frac{\partial \Phi}{\partial v}\left(s t \tilde{v}_{0}, \kappa\right) \tilde{v}_{0} d t
\end{aligned}
$$

Assim, vamos obter soluções não-triviais $(s \neq 0)$ da equação (2.39) pela solução de

$$
\tilde{\Phi}(s, \kappa) \equiv 0, s \in(-\delta, \delta)
$$

De fato, pela hipótese $(2.33), \tilde{\Phi} \in C^{1}\left((-\delta, \delta) \times \tilde{V}, Z_{0}\right)$, e pelo Corolário 2.3.1.1 $\tilde{\Phi}\left(0, \kappa_{0}\right)=0$. A seguir veremos que $\frac{\partial \tilde{\Phi}}{\partial \kappa}\left(0, \kappa_{0}\right) \neq 0$. De fato, usando a regra da cadeia não é difícil ver que

$$
\begin{aligned}
\frac{\partial \tilde{\Phi}}{\partial \kappa}\left(0, \kappa_{0}\right)= & Q \frac{\partial^{2} F}{\partial x^{2}}\left(0, \kappa_{0}\right)\left[\tilde{v}_{0}+\frac{\partial \psi}{\partial v}\left(0, \kappa_{0}\right) \tilde{v}_{0}, \frac{\partial \psi}{\partial \kappa}\left(0, \kappa_{0}\right)\right] \\
& +Q \frac{\partial^{2} F}{\partial x \partial \kappa}\left(0, \kappa_{0}\right)\left(\tilde{v}_{0}+\frac{\partial \psi}{\partial v}\left(0, \kappa_{0}\right) \tilde{v}_{0}\right)+Q \frac{\partial F}{\partial x}\left(0, \kappa_{0}\right) \frac{\partial^{2} \psi}{\partial \kappa \partial v}\left(0, \kappa_{0}\right) \tilde{v}_{0}
\end{aligned}
$$

Assim, em virtude de (2.40), Corolário 2.3.1.1 e (2.37), obtemos

$$
\frac{\partial \tilde{\Phi}}{\partial \kappa}\left(0, \kappa_{0}\right)=Q \frac{\partial^{2} F}{\partial x \partial \kappa}\left(0, \kappa_{0}\right) \tilde{v}_{0}=Q \frac{\partial^{2} F}{\partial x \partial \kappa}\left(0, \kappa_{0}\right) \tilde{v}_{0} \neq 0 \in Z_{0}
$$

Portanto, temos que $\tilde{\Phi}\left(0, \kappa_{0}\right)=0$ e $\frac{\partial \tilde{\Phi}}{\partial \kappa}\left(0, \kappa_{0}\right) \neq 0$, então podemos aplicar o Teorema da Função Implícita e assim, existe uma função diferenciável $\varphi:(-\delta, \delta) \rightarrow \tilde{V}, s \mapsto \kappa=\varphi(s)$, tal que 
$\varphi(0)=\kappa_{0}, \tilde{\Phi}(s, \varphi(s))=0$, para todo $s \in(-\delta, \delta)$. Dessa forma, para $\kappa=\varphi(s)$, temos

$$
\Phi\left(s \tilde{v}_{0}, \varphi(s)\right)=Q F\left(s \tilde{v}_{0}+\psi\left(s \tilde{v}_{0}, \varphi(s)\right), \varphi(s)\right)=s \tilde{\Phi}(s, \kappa)=0, \forall s \in(-\delta, \delta)
$$

o qual implica que $F(x(s), \kappa(s))=0$, para

$$
x(s)=s \tilde{v}_{0}+\psi\left(s \tilde{v}_{0}, \kappa(s)\right), \quad \kappa(s)=\varphi(s)
$$

$\log o \mapsto(x(s), \kappa(s))$ é a curva procurada.

Observação 2.3.1. Da demonstração acima $x(s)$ temos que para todo $s, x(s)$ tem uma decomposiçấo de ortogonal, pois $x(s)=s \tilde{v}_{0}+\psi\left(s \tilde{v}_{0}, \kappa(s)\right)$, com s $\tilde{v}_{0} \in \mathscr{N}\left(\frac{\partial F}{\partial x}\left(0, \kappa_{0}\right)\right)$ e $\psi\left(s \tilde{v}_{0}, \kappa(s)\right) \in$ $\mathscr{R}\left(\frac{\partial F}{\partial x}\left(0, \kappa_{0}\right)\right)=\mathscr{N}\left(\frac{\partial F}{\partial x}\left(0, \kappa_{0}\right)\right)^{\perp}$.

Observação 2.3.2. Se no Teorema 2.3.2 supomos que $F \in C^{k}(U \times V), k \geq 2$, então a curva

$$
s \in(-\delta, \delta) \mapsto(x(s), \kappa(s))
$$

é de classe $C^{k-1}$ em $(-\delta, \delta)$.

Corolário 2.3.2.1. O vetor tangente da curva solução não-trivial (2.38) no ponto de bifurcação $\left(0, \kappa_{0}\right)$ é dado por

$$
\left(\tilde{v}_{0}, \dot{\kappa}(0)\right) \in X \times \mathbb{R}
$$

onde $" * "=\frac{d}{d s}$.

Demonstração: Do teorema temos $x(s)=s \tilde{v}_{0}+\psi\left(s \tilde{v}_{0}, \kappa(s)\right)$ e $\kappa(s)=\varphi(s)$; ou seja, do Corolário 2.3.1.1 e de (2.40),

$$
\left.\frac{d}{d s}(x(s))\right|_{s=0}=\tilde{v}_{0}+\frac{\partial \psi}{\partial v}\left(0, \kappa_{0}\right) \tilde{v}_{0}+\frac{\partial \psi}{\partial \kappa}\left(0, \kappa_{0}\right) \dot{\kappa}(0)=\tilde{v}_{0}
$$

A seguir daremos um simples exemplo como aplicar o Teorema 2.3.2. Claramente, observamos que $u(x)=0, x \in[0, L]$, é uma solução do problema (2.47) abaixo. Usando o Teorema 2.3.2, mostraremos que existe a curva $s \in(-\delta, \delta) \mapsto(x(s), \kappa(s))$, com $(x(0), \kappa(0))=\left(0, \kappa_{n}\right)$, onde $\kappa_{n}=\left(\frac{n \pi}{L}\right)^{2}$, para todo $n \in \mathbb{N}$, tal que $x^{\prime \prime}(s)+\kappa(s) \sin (x(s))=0$; isto é, $\left(0, \kappa_{n}\right)$ é um ponto de bifurcação.

Exemplo 2.3.1. Consideremos o seguinte problema

$$
\begin{aligned}
& u^{\prime \prime}(x)+\kappa \sin (u(x))=0, \quad \forall x \in[0, L] \\
& u^{\prime}(0)=u^{\prime}(L)=0,
\end{aligned}
$$


onde $L$ está fixo e $\kappa>0$ é nosso parâmetro. Sejam $X=\left\{u \in C^{2}([0, L]): u^{\prime}(0)=u^{\prime}(L)=0\right\}$, $Z=C([0, L])$ e a aplicação

$$
\begin{aligned}
F: X \times \mathbb{R} & \longrightarrow Z \\
(u, \kappa) & \longmapsto F(u, \kappa)=u^{\prime \prime}+\kappa \sin u .
\end{aligned}
$$

Então, $F \in C^{2}(X \times \mathbb{R}, Z)$ e $F(0, \kappa)=0$, para todo $\kappa \in \mathbb{R}$. Agora verificamos (2.35), (2.36) e (2.37) $\operatorname{para} \mathcal{L}_{u}=\frac{\partial F}{\partial u}(u, \kappa)=\frac{d^{2}}{d x^{2}}+\kappa \cos u$.

Para $(u, \kappa)=(0, \kappa), \kappa \in \mathbb{R}$, temos $\mathcal{L}_{0} f=f^{\prime \prime}+\kappa f$. Como $\kappa>0, \mathcal{L}_{0} f=0$ se, e somente se, $f(x)=c \cos (\sqrt{\kappa} x)+d \sin (\sqrt{\kappa} x)$. Mais ainda, $f^{\prime}(0)=0$, assim $d=0 ; \operatorname{logo}, f(x)=$ $c \cos (\sqrt{\kappa} x)$. Agora, para $c \neq 0, f^{\prime}(L)=0$ se, e somente se, $\kappa_{n}=\left(\frac{n \pi}{L}\right)^{2}, n \in \mathbb{N}$. Portanto, $\mathcal{L}_{0} f=0, \operatorname{com} f^{\prime}(0)=f^{\prime}(L)=0$, tem solução $(f, \kappa)$, onde $\kappa>0$ e $f \neq 0$, se, e somente se, $\kappa \in$ $\left\{\kappa_{n}=\left(\frac{n \pi}{L}\right)^{2} \mid n \in \mathbb{N}\right\}$. Desta maneira, $\mathcal{L}_{0} u=0$ se, e somente se, $u(x)=c u_{n}(x)$, onde $u_{n}(x)=$ $\cos \frac{n \pi x}{L}$, com $\mathscr{N}\left(\frac{\partial F}{\partial u}\left(0, \kappa_{n}\right)\right)=\left[u_{n}\right]$. Determinemos a codim $\mathscr{R}\left(\frac{\partial F}{\partial u}\left(0, \kappa_{n}\right)\right)$. Suponhamos que $h \in \mathscr{R}\left(\frac{\partial F}{\partial u}\left(0, \kappa_{n}\right)\right)$, logo $u^{\prime \prime}(x)+\kappa u(x)=h(x)$, onde $u \in X$ e $h \in Z$. Como $\mathcal{L}_{0}$ é um operador autoadjunto, temos que $\mathscr{N}\left(\mathcal{L}_{0}\right)^{\perp}=\overline{\mathscr{R}\left(\mathcal{L}_{0}\right)}=\mathscr{R}\left(\mathcal{L}_{0}\right)$. Logo, $h \in \mathscr{R}\left(\mathcal{L}_{0}\right)$ se, e somente se, $h \perp u_{n}$, isto é,

$$
\mathscr{R}\left(\frac{\partial F}{\partial u}\left(0, \kappa_{n}\right)\right)=\left\{h \in Z \mid \int_{0}^{L} h(x) u_{n}(x) d x=0\right\}
$$

ainda mais,

$$
1=\operatorname{dim} \mathscr{N}\left(\frac{\partial F}{\partial u}\left(0, \kappa_{n}\right)\right)=\operatorname{dim} \mathscr{R}\left(\frac{\partial F}{\partial u}\left(0, \kappa_{n}\right)\right)^{\perp}=\operatorname{codim} \mathscr{R}\left(\frac{\partial F}{\partial u}\left(0, \kappa_{n}\right)\right)
$$

Finalmente, $\frac{\partial^{2} F}{\partial \kappa \partial u}(u, \kappa)=\cos u$. Como $\int_{0}^{L}\left|u_{n}(x)\right|^{2} d x \neq 0, \frac{\partial^{2} F}{\partial \kappa \partial u}\left(0, \kappa_{n}\right) u_{n}=u_{n} \notin \mathscr{R}\left(\frac{\partial F}{\partial x}\left(0, \kappa_{n}\right)\right)$. Portanto, o Teorema de Crandall-Rabinowitz implica que, para todo $n \in \mathbb{N},\left(0, \kappa_{k}\right)$ é um ponto de bifurcação para o problema (2.47) e assim existe curva $s \in(-\delta, \delta) \mapsto(x(s), \kappa(s))$, com $(x(0), \kappa(0))=\left(0, \kappa_{n}\right)$ tal que $x^{\prime \prime}(s)+\kappa(s) \sin (x(s))=0$. 


\section{Propriedades da Equação NLS- $\delta$}

Consideramos o modelo NLS- $\delta$

$$
\left\{\begin{array}{l}
i \frac{\partial u}{\partial t}=H_{q} u-\lambda|u|^{p} u, \quad(x, t) \in \mathbb{R} \times \mathbb{R}, \\
u(0)=u_{0}
\end{array}\right.
$$

onde $\lambda \in \mathbb{R}, H_{q}=-\frac{1}{2} \Delta+q \delta(x), q<0$, com $\delta$ denotando a distribuição Delta de Dirac e $0 \leq p<\infty$.

Neste capítulo iniciamos estudando dois pontos básicos na dinâmica do modelo (3.1): a boa colocação local e global de NLS- $\delta$ em $H^{1}(\mathbb{R})$ e a fórmula explícita para o grupo determinado por $H_{q}$. Em seguida, utilizando as componentes pontuais e contínuas veremos a aplicabilidade das técnicas de Variedades Invariantes de equações diferenciais ordinárias (veja [9]) para a classe de equações diferenciais parciais do tipo de Schrödinger não-linear. Embora os métodos de Variedades Invariantes são usados, em geral, no estudo da evolução de tempo de EDP's de tipo dissipativa, veremos como é possível os aplicar ao caso de equação dispersivas não-lineares. Finalizamos, apresentando algumas estimativas dispersivas do grupo $\left\{e^{i t H_{q}}\right\}_{t \in \mathbb{R}}$.

\subsection{Boa colocação em $H^{1}(\mathbb{R})$}

Nesta seção mostraremos que o problema de boa colocação local para (3.1) é bem posto no espaço $H^{1}(\mathbb{R})$. Além disso, dependendo da potência $p$, podemos determinar resultados sobre o problema de existência global.

Iniciamos lembrando que a equação NLS- $\delta$ tem duas quantidades conservadas,

$$
\begin{aligned}
E(u) & =\frac{1}{2}\left\|u_{x}\right\|_{2}^{2}-\frac{2 \lambda}{p+2}\|u\|_{p+2}^{p+2}-q|u(0)|^{2} \\
Q(u) & =\int|u(x)|^{2} d x
\end{aligned}
$$

A saber, $E(u(t))=E(u(0))$ e $Q(u(t))=Q(u(0))$, para todo $t \in[0, T]$. Assim, podemos escrever formalmente a equação (3.1) em forma Hamiltoniana

$$
i u_{t}=E^{\prime}(u(t))
$$

onde $E^{\prime}$ representa a derivada de Frechet de $E$. Os funcionais $E$ e $Q$ estão bem definidos em 
$H^{1}(\mathbb{R})$. É, portanto, natural construir localmente o fluxo para (3.1) com uma condição inicial na classe $H^{1}(\mathbb{R})$ e assim deduzir a existência global para esses dados.

A seguir, apresentamos duas provas sobre o problema de boa colocação local em $H^{1}(\mathbb{R})$ para (3.1), a primeira é baseada no Teorema 3.7.1 em [11] e a segunda é baseada na Teoria de Operadores de Onda $([15])$.

Proposição 3.1. Sejam $q \neq 0$ em (3.1) e $\lambda \in \mathbb{R}, \operatorname{com} \lambda \neq 0$, o problema é localmente me posto; isto é, para $u_{0} \in H^{1}(\mathbb{R})$, existe $T=T\left(\left\|u_{0}\right\|_{H^{1}(\mathbb{R})}\right)>0$ e uma única solução $u \in C\left([0, T), H^{1}(\mathbb{R}, \mathbb{C})\right)$ tal que $u(0)=u_{0}$. Ainda mais, para todo $T^{\prime}<T$, existe $B\left(u_{0} ; \delta\right)$ tal que a aplicação dado-solução

$$
u_{0} \in B\left(u_{0} ; \delta\right) \mapsto u \in C\left(\left[0, T^{\prime}\right] ; H^{1}(\mathbb{R})\right)
$$

é contínua. Além disso, a energia $E$ e a carga $Q$ são conservadas.

Demonstração: De fato, pelo Teorema 2.1.4, temos que o operador $H_{q} \geqq-\beta$, onde $\beta=\frac{q^{2}}{2}$, se $q<0$ e $\beta=0$, se $q>0$. Assim, para o operador autoadjunto $\mathscr{A} \equiv-H_{q}-\beta$ sobre o espaço $X=L^{2}(\mathbb{R})$ com domínio $\mathscr{D}(\mathscr{A})=\mathscr{D}\left(H_{q}\right)$ temos que $\mathscr{A} \leqq 0$. Além disso, em nosso caso é possível considerar o espaço $X_{\mathscr{A}}=H^{1}(\mathbb{R})$ com norma

$$
\|u\|_{X \mathscr{A}}^{2}=\left\|u_{x}\right\|_{2}^{2}+(\beta+1)\|u\|_{2}^{2}+q|u(0)|^{2}
$$

Veja, para $q>0,\|u\|_{X_{\mathscr{A}}}^{2}=\left\|u_{x}\right\|_{2}^{2}+\|u\|_{2}^{2}+q|u(0)|^{2} \leq\left\|u_{x}\right\|_{2}^{2}+\|u\|_{2}^{2}+q C\|u\|_{H^{1}}^{2} \leq C(q)\|u\|_{H^{1}}^{2} \mathrm{e}$ $\|u\|_{H^{1}}^{2} \leq\left\|u_{x}\right\|_{2}^{2}+\|u\|_{2}^{2}+q|u(0)|^{2}=\|u\|_{X \mathscr{A}^{\prime}}^{2}$ para $q<0,\|u\|_{X_{\mathscr{A}}}^{2}=\left\|u_{x}\right\|_{2}^{2}+(\beta+1)\|u\|_{2}^{2}+q|u(0)|^{2} \leq$ $\left\|u_{x}\right\|_{2}^{2}+(\beta+1)\|u\|_{2}^{2} \leq(\beta+1)\|u\|_{H^{1}}^{2}$ e como $|u(0)| \leq C\|u\|_{H^{1}}$ e $\beta+q+1>0$, para todo $q$, $\|u\|_{H^{1}}^{2} \leq(\beta+q+1)\|u\|_{H^{1}}^{2} \leq(\beta+1)\|u\|_{H^{1}}^{2}+q\|u\|_{H^{1}}^{2} \leq C_{1}\left(\left\|u_{x}\right\|_{2}^{2}+(\beta+1)\|u\|_{2}^{2}+q|u(0)|^{2}\right)=$ $C_{1}\|u\|_{X \mathscr{A}}^{2}$ onde $C_{1}=\max \left\{\beta, \frac{1}{C}\right\}$. Logo a norma definida em $X_{\mathscr{A}}$ é equivalente à norma usual de $H^{1}(\mathbb{R})$. Temos que $X_{\mathscr{A}}=H^{1}(\mathbb{R}) \hookrightarrow L^{2}(\mathbb{R})$. Chame $g(u)=\lambda|u|^{p} u$. Assim $g \in C\left(X_{\mathscr{A}}, L^{2}(\mathbb{R})\right)$ e $\|g(v)-g(u)\|_{2} \leq C\left(\|v\|_{X \mathscr{A}}^{p}+\|v\|_{X \mathscr{A}}^{p}\right)\|v-u\|_{2}$. Além disso, $g=G^{\prime}$, onde $G(u)=\frac{\lambda}{p+2}|u|^{p+2} \mathrm{e}$ $G \in C^{1}\left(X_{\mathscr{A}}, \mathbb{R}\right)$. Mais ainda, para toda $u \in X_{\mathscr{A}}, \operatorname{Im}(g(u) \bar{u})=\operatorname{Im}\left(\lambda|u|^{p+1}\right)=0$ em $\mathbb{R}$. Logo, a unicidade de soluções e as condições (3.7.1), (3.7.3)-(3.7.6) em [11] são satisfeitas com $r=\rho^{\prime}=2$. Finalmente, a condição (3.7.2) em [11] é válida, pois $\mathscr{A}$ é um operador autoadjunto no espaço $L^{2}(\mathbb{R})$. Portanto, por [11, Teorema 3.7.1] o problema de valor inicial (3.1) é localmente bem posto em $H^{1}(\mathbb{R})$.

A seguir apresentamos outra demonstração do problema de boa colocação em $H^{1}(\mathbb{R})$ para (3.1) baseada na Teoria de Operadores de Onda (veja [15]). Para isto consideremos a equação de Duhamel associada ao problema (3.1)

$$
u(t)=U_{q}(t) u_{0}+i \lambda \int_{0}^{t} U_{q}(t-s)|u(s)|^{p} u(s) d s,
$$


onde $U_{q}(t)$ é o grupo unitário gerado por $H_{q}$ (Ver Lema 3.2.1 abaixo)

$$
U_{q}(t) \equiv e^{-i H_{q} t}
$$

Teorema 3.1.1. O problema (3.1) é localmente bem posto em $H^{1}(\mathbb{R})$.

Demonstração: Para mostrar a existência de uma solução para (3.5) em $H^{1}(\mathbb{R})$, devemos provar a existência de um ponto fixo da aplicação

$$
J[u](t)=U_{q}(t) u_{0}+i \lambda \int_{0}^{t} U_{q}(t-s)|u(s)|^{p} u(s) d s,
$$

onde $J: C\left([0, T], H^{1}(\mathbb{R})\right) \longrightarrow C\left([0, T], H^{1}(\mathbb{R})\right)$ será contínua, para algum $T>0$.

Para limitar $J[u]$ e sua derivada primeira em $L^{2}$, introduzimos o operador $\mathcal{A}=I+H_{q} P_{c}$, onde $P_{c}$ denota a projeção sobre a parte espectral contínua de $H_{q}$ (Ver a fórmula 3.30 abaixo).

Note que $\mathcal{A}$ é um operador não negativo, pois pelo Teorema 2.1.4 o espetro contínuo associado a $H_{q}$ é dado por $[0, \infty)$, logo existe $\mathcal{A}^{1 / 2}$. De fato, pelo Teorema espectral temos $L^{2}(\mathbb{R})=$ $\left[\psi_{0}\right] \oplus \mathcal{H}_{c}\left(H_{q}\right)$, onde $\mathcal{H}_{c}\left(H_{q}\right)=\mathscr{R}\left(P_{c} H_{q}\right)$, assim para $u \in \mathscr{D}\left(H_{q}\right)$, tal que $u=u_{p} \psi_{0}+u_{c}$, com $\left\langle H_{q} u_{c}, u_{c}\right\rangle \geq d\left\|u_{c}\right\|_{2}^{2}$, temos

$$
\langle\mathcal{A} u, u\rangle=\left\langle u+H_{q} P_{c} u, u\right\rangle=\langle u, u\rangle+\left\langle H_{q} u_{c}, u_{c}\right\rangle \geq\|u\|_{2}^{2}+d\left\|u_{c}\right\|_{2}^{2} \geq d_{0}\|u\|_{2}^{2}, \quad d_{0}>0 .
$$

Além disso, $\left.H_{q}\left(\mathcal{H}_{c}\left(H_{q}\right)\right) \cap \mathscr{D}\left(H_{q}\right)\right) \subseteq \mathcal{H}_{c}\left(H_{q}\right)$. De [15], temos $W_{ \pm} W_{ \pm}^{*}=P_{c}, W_{ \pm}^{*} W_{ \pm}=I, \mathcal{A}^{1 / 2}=$ $W_{ \pm}\left(I-\partial_{x}^{2}\right)^{\frac{1}{2}} W_{ \pm}^{*}$ e $W_{ \pm}^{*} \mathcal{A}^{1 / 2} W_{ \pm}=\left(I-\partial_{x}^{2}\right)^{\frac{1}{2}}$, onde $W_{ \pm} \equiv s-\lim _{t \rightarrow \pm \infty} e^{i t H_{q}} e^{-i t H_{0}}$ e $W_{ \pm}^{*} \equiv s-$ $\lim _{t \rightarrow \pm \infty} e^{i t H_{0}} e^{-i t H_{q}} P_{c}$. Mais ainda, $\left\|\mathcal{A}^{1 / 2} f\right\|_{2} \sim\|f\|_{H^{1}}=\left\|\left(I-\partial_{x}^{2}\right)^{1 / 2} f\right\|_{2}$. De fato, observe que

$$
\begin{aligned}
& f \in L^{2}(\mathbb{R}) \rightarrow\left(I-\partial_{x}^{2}\right)^{-1 / 2} f \in H^{1}(\mathbb{R}) \rightarrow \mathcal{A}^{1 / 2}\left(I-\partial_{x}^{2}\right)^{-1 / 2} f \in L^{2}(\mathbb{R}), \\
& f \in L^{2}(\mathbb{R}) \rightarrow\left(I-\partial_{x}^{2}\right)^{1 / 2} f \in H^{-1}(\mathbb{R}) \rightarrow \mathcal{A}^{-1 / 2}\left(I-\partial_{x}^{2}\right)^{1 / 2} f \in L^{2}(\mathbb{R}) .
\end{aligned}
$$

Assim,

$$
\begin{aligned}
\left\|\mathcal{A}^{1 / 2}\left(I-\partial_{x}^{2}\right)^{-1 / 2} f\right\|_{2} & =\left\|W_{ \pm}\left(I-\partial_{x}^{2}\right)^{\frac{1}{2}} W_{ \pm}^{*}\left(I-\partial_{x}^{2}\right)^{-1 / 2} f\right\|_{2} \\
& \leq C\left\|\left(I-\partial_{x}^{2}\right)^{\frac{1}{2}} W_{ \pm}^{*}\left(I-\partial_{x}^{2}\right)^{-1 / 2} f\right\|_{2}=C\left\|W_{ \pm}^{*}\left(I-\partial_{x}^{2}\right)^{-1 / 2} f\right\|_{H^{1}} \\
& \leq C_{1}\left\|\left(I-\partial_{x}^{2}\right)^{-1 / 2} f\right\|_{H^{1}}=C_{1}\|f\|_{2} .
\end{aligned}
$$

Logo os operadores $\mathcal{A}^{1 / 2}\left(I-\partial_{x}^{2}\right)^{-1 / 2}$ e $\mathcal{A}^{-1 / 2}\left(I-\partial_{x}^{2}\right)^{1 / 2}$ são limitados de $L^{2}(\mathbb{R})$ em $L^{2}(\mathbb{R})$. Então

$$
\begin{aligned}
\left\|\mathcal{A}^{1 / 2} f\right\|_{2} & =\left\|\mathcal{A}^{1 / 2}\left(I-\partial_{x}^{2}\right)^{-1 / 2}\left(I-\partial_{x}^{2}\right)^{1 / 2} f\right\|_{2} \\
& \leq\left\|\mathcal{A}^{1 / 2}\left(I-\partial_{x}^{2}\right)^{-1 / 2}\right\|\left\|\left(I-\partial_{x}^{2}\right)^{1 / 2} f\right\|_{2} \leq C_{2}\|f\|_{H^{1}}
\end{aligned}
$$


por outro lado,

$$
\begin{aligned}
\|f\|_{H^{1}} & =\left\|\left(I-\partial_{x}^{2}\right)^{1 / 2} f\right\|_{2}=\left\|\mathcal{A}^{-1 / 2}\left(I-\partial_{x}^{2}\right)^{1 / 2} \mathcal{A}^{1 / 2} f\right\|_{2} \\
& \leq\left\|\mathcal{A}^{-1 / 2}\left(I-\partial_{x}^{2}\right)^{1 / 2}\right\|\left\|\mathcal{A}^{1 / 2} f\right\|_{2} \leq C_{3}\left\|\mathcal{A}^{1 / 2} f\right\|_{2}
\end{aligned}
$$

Portanto, temos uma equivalência de normas

$$
C_{1}\|f\|_{H^{1}} \leq\left\|\mathcal{A}^{1 / 2} f\right\|_{2} \leq C_{2}\|f\|_{H^{1}} .
$$

Definimos o operador $\mathcal{A}$, pois $\mathcal{A}^{1 / 2}$ comuta com o propagador $e^{-i H_{q} t}$. De fato,

$$
\begin{aligned}
\mathcal{A}^{1 / 2} e^{-i H_{q} t}(g) & =W_{ \pm}\left(I-\partial_{x}^{2}\right)^{\frac{1}{2}} W_{ \pm}^{*} e^{-i H_{q} t}(g) \\
& =\lim _{\tau \rightarrow \pm \infty} \lim _{\mu \rightarrow \pm \infty} e^{i H_{q} \tau} e^{-i H_{0} \tau}\left(I-\partial_{x}^{2}\right)^{\frac{1}{2}} e^{i H_{0} \mu} e^{-i H_{q} \mu} P_{c} e^{-i H_{q} t}(g) \\
& =e^{-i H_{q} t} \lim _{\tau \rightarrow \pm \infty} \lim _{\mu \rightarrow \pm \infty} e^{i H_{q} \tau} e^{-i H_{0} \tau}\left(I-\partial_{x}^{2}\right)^{\frac{1}{2}} e^{i H_{0} \mu} e^{-i H_{q} \mu} P_{c}(g)=e^{-i H_{q} t} \mathcal{A}^{1 / 2}(g)
\end{aligned}
$$

Em geral, a notação $\mathcal{J}^{s}=\left(I-\partial_{x}^{2}\right)^{s / 2}$ é muito usada, que é o potencial de Bessel, logo $\mathcal{J}=\left(I-\partial_{x}^{2}\right)^{1 / 2}$. Então, da desigualdade de Kato-Ponce temos que

$$
\begin{aligned}
\|\mathcal{J}(f g)\|_{2} & \leq C_{1}\left(\|f\|_{\infty}\|g\|_{2}+\|f\|_{2}\|g\|_{\infty}\right) \\
& \leq C_{1}\left(\|f\|_{\infty}\|g\|_{H^{1}}+\|f\|_{H^{1}}\|g\|_{\infty}\right) \\
& =C_{1}\left(\|f\|_{\infty}\|\mathcal{J} g\|_{2}+\|\mathcal{J} f\|_{2}\|g\|_{\infty}\right) .
\end{aligned}
$$

Seja $X=C\left([0, T], H^{1}(\mathbb{R})\right)$. É fácil ver que para $u \in X$, temos $J[u] \in X$ e da teoria de grupos $t \mapsto J[u](t)$ é contínua. Agora, como $\mathcal{A}^{1 / 2}$ comuta com $U_{q}(t)$, temos

$$
\mathcal{A}^{1 / 2} J[u](t)=U_{q}(t) \mathcal{A}^{1 / 2} u_{0}+i \lambda \int_{0}^{t} U_{q}(t-s) \mathcal{A}^{1 / 2}\left(|u(s)|^{p} u(s)\right) d s .
$$

Assim, como $\left\|U_{q}(t)\right\|_{2}=1$,

$$
\begin{aligned}
\left\|\mathcal{A}^{1 / 2} J[u]\right\|_{2} & \leq\left\|\mathcal{A}^{1 / 2} u_{0}\right\|_{2}+|\lambda| \int_{0}^{t}\left\|\mathcal{A}^{1 / 2}\left(|u(s)|^{p} u(s)\right)\right\|_{2} d s \\
& =\left\|\mathcal{A}^{1 / 2} u_{0}\right\|_{2}+|\lambda| \int_{0}^{t}\left\|\mathcal{A}^{1 / 2} \mathcal{J}^{-1} \mathcal{J}\left(|u(s)|^{p} u(s)\right)\right\|_{2} d s
\end{aligned}
$$

De (3.8) e (3.10)

$$
\begin{aligned}
\|J[u](t)\|_{H^{1}} & \leq C\left\|\mathcal{A}^{1 / 2} J[u]\right\|_{2} \\
& \leq C\left\|u_{0}\right\|_{H^{1}}+C\left\|\mathcal{A}^{1 / 2} \mathcal{J}^{-1}\right\| \int_{0}^{t}\left\|\mathcal{J}\left(|u(s)|^{p} u(s)\right)\right\|_{2} d s
\end{aligned}
$$


mas, de (3.9), da imersão de Sobolev e $H^{s}(\mathbb{R}), s>\frac{1}{2}$, sendo uma álgebra de Banach, temos

$$
\begin{aligned}
\left\|\mathcal{J}\left(|u|^{p} u\right)\right\|_{2} & \leq C_{1}\left(\left\||u|^{p}\right\|_{\infty}\|\mathcal{J} u\|_{2}+\left\|\mathcal{J}\left(|u|^{p}\right)\right\|_{2}\|u\|_{\infty}\right) \\
& =C_{1}\left(\|u\|_{H^{1}}^{p}\|u\|_{H^{1}}+\|u\|_{H^{1}}^{p}\|u\|_{H^{1}}\right) \\
& =2 C_{1}\|u\|_{H^{1}}^{p+1}
\end{aligned}
$$

substituindo na desigualdade anterior

$$
\begin{aligned}
\|J[u](t)\|_{H^{1}} & \leq C\left\|u_{0}\right\|_{H^{1}}+C M \int_{0}^{t} 2 C_{1}\|u(s)\|_{H^{1}}^{p+1} d s \\
& \leq C\left\|u_{0}\right\|_{H^{1}}+C_{2} M T \sup _{s \in[0, T]}\|u(s)\|_{H^{1}}^{p+1} .
\end{aligned}
$$

Denotaremos, $\|u\|_{X}=\sup _{s \in[0, T]}\|u(s)\|_{H^{1}}$. Como $u \in X$, então $\|J[u]\|_{X} \leq C\left\|u_{0}\right\|_{H^{1}(\mathbb{R})}+C_{3} T\|u\|_{X}^{p+1}<$ $\infty, \log \sigma[u] \in X$.

Agora, afirmamos que para a bola $B\left(0 ; 2 C\left\|u_{0}\right\|_{H^{1}}\right)=\left\{u \in X:\|u\|_{X} \leq 2 C\left\|u_{0}\right\|_{H^{1}}\right\}$, $J\left(B\left(0 ; 2 C\left\|u_{0}\right\|_{H^{1}}\right)\right) \subseteq B\left(0 ; 2 C\left\|u_{0}\right\|_{H^{1}}\right)$ para um $T$ específico. Suponhamos que $u$ é tal que $\|u\|_{X} \leq$ $2 C\left\|u_{0}\right\|_{H^{1}}$. Então tomando $T$ suficientemente pequeno, tal que $2^{p+1} C_{3} T C^{p}\left\|u_{0}\right\|_{H^{1}}^{p}<1$, temos

$$
\|J[u](t)\|_{H^{1}} \leq C\left\|u_{0}\right\|_{H^{1}}+C_{3} T\left(2 C\left\|u_{0}\right\|_{H^{1}}\right)^{p+1} \leq C\left\|u_{0}\right\|_{H^{1}}+C\left\|u_{0}\right\|_{H^{1}}=2 C\left\|u_{0}\right\|_{H^{1}} .
$$

Logo, $\|J[u]\|_{X} \leq 2 C\left\|u_{0}\right\|_{H^{1}}$. Segue que para $T$ suficientemente pequeno, segue a afirmação. Veremos a seguir, que $J$ é uma contração. Sejam $u, v \in H^{1}(\mathbb{R})$ tais que $\|u\|_{X} \leq 2 C\left\|u_{0}\right\|_{H^{1}}$ e $\|v\|_{X} \leq 2 C\left\|u_{0}\right\|_{H^{1}}$. Então, pela equivalência (3.8),

$$
\begin{aligned}
\|J[u](t)-J[v](t)\|_{H^{1}} & \leq C\left\|\mathcal{A}^{1 / 2}(J[u]-J[v])\right\|_{2} \\
& \leq|\lambda| \int_{0}^{t} \|\left(\mathcal{A}^{1 / 2} \mathcal{J}^{-1} \mathcal{J}\left(|u(s)|^{p} u(s)-|v(s)|^{p} v(s)\right) \|_{2} d s\right. \\
& \leq K|\lambda| \int_{0}^{t}\left\||u(s)|^{p} u(s)-|v(s)|^{p} v(s)\right\|_{H^{1}} d s \\
& \leq K|\lambda| C_{0} \int_{0}^{t}\left(\|u(s)\|_{H^{1}}^{p}+\|v(s)\|_{H^{1}}^{p}\right)\|u(s)-v(s)\|_{H^{1}} d s \\
& \leq K|\lambda|\left(2 C\left\|u_{0}\right\|_{H^{1}}\right)^{p} \int_{0}^{t}\|u(s)-v(s)\|_{H^{1}} d s,
\end{aligned}
$$

e portanto,

$$
\|J[u]-J[v]\|_{X} \leq K|\lambda| T\left(2 C\left\|u_{0}\right\|_{H^{1}}\right)^{p}\|u-v\|_{X}
$$

Desta forma, fazendo outra escolha de $T$ tal que $K|\lambda| T\left(2 C\left\|u_{0}\right\|_{H^{1}}\right)^{p}<1$, temos que a transformação $J\left[\right.$.] é uma contração na bola $B\left(0 ; 2 C\left\|u_{0}\right\|\right)$ no espaço $X$ para $T$ pequeno. Logo, existe $u \in B\left(0 ; 2 C\left\|u_{0}\right\|\right) \subseteq C\left([0, T] ; H^{1}(\mathbb{R})\right)$, para $T$ pequeno, tal que $J(u)=u$. Assim, $u=u(t)$ satisfaz a equação integral (3.5).

Provemos agora a dependência contínua da solução com respeito ao dado inicial, ou seja, provaremos que para todo $T^{\prime}<T$, existe uma vizinhança $V$ de $u_{0}$ em $H^{1}(\mathbb{R})$ tal que a função 
$J: V \rightarrow C\left(\left[T^{\prime}, T^{\prime}\right], H^{1}(\mathbb{R})\right)$ é lipschitziana. Com efeito, sejam $u$, $v$ soluções da equação integral (3.5) com dados iniciais $u_{0}, v_{0}$, então, conforme já vimos

$$
\|u-v\|_{X} \leq C\left\|u_{0}-v_{0}\right\|_{H^{1}(\mathbb{R})}+K|\lambda| T\left(2 C\left\|u_{0}\right\|_{H^{1}}\right)^{p}\|u-v\|_{X}
$$

ou seja,

$$
\left(1-K|\lambda| T\left(2 C\left\|u_{0}\right\|_{H^{1}}\right)^{p}\right)\|u-v\|_{X} \leq C\left\|u_{0}-v_{0}\right\|_{H^{1}(\mathbb{R})} .
$$

Seja $T^{\prime}<T_{\dot{C}}$ Da escolha de $T$ segue que $\left(1-K|\lambda| T^{\prime}\left(2 C\left\|u_{0}\right\|_{H^{1}}\right)^{p}\right)>0$. Logo, $\|u-v\|_{X} \leq$ $\frac{C}{\left(1-K|\lambda| T^{\prime}\left(2 C\left\|u_{0}\right\|_{H^{1}}\right)^{p}\right)}\left\|u_{0}-v_{0}\right\|_{H^{1}(\mathbb{R})}=\widetilde{K}\left\|u_{0}-v_{0}\right\|_{H^{1}(\mathbb{R})}$, isto é, $J$ é lipschitziana.

Observação 3.1.1. Agora veremos formalmente que a energia $E$ e a carga $Q$ em (3.2) e (3.3) são conservadas:

$$
\begin{aligned}
& E(u(t))=E\left(u_{0}\right), \\
& Q(u(t))=Q(u(0)),
\end{aligned}
$$

onde $u(t)$ é a solução obtida no Teorema 3.1.1 para $t \in[0, T]$. De fato, suponhamos que $u_{0} \in H^{1}(\mathbb{R})$. Seja $\left(u_{0}^{k}\right)_{k \in \mathbb{N}} \subset \mathcal{S}(\mathbb{R})$ uma sequência de funções tais que $\left\|u_{0}^{k}-u_{0}\right\|_{H^{1}} \rightarrow 0$, quando $k \rightarrow \infty$. Pelo Teorema 3.1.1 existe $T>0$ tal que $u^{k} \in C\left([-T, T] ; H^{1}(\mathbb{R})\right), k=1,2, \ldots$ é solução da equação integral (3.5) e problema (3.1) com valor inicial $u_{0}^{k}$. Além disso, $\left\|u^{k}(t)\right\|_{2}=\left\|u_{0}^{k}\right\|_{2}$ e $E\left(u^{k}(t)\right)=$ $E\left(u_{0}^{k}\right)$ para todo $t \in[-T, T]$. Da dependência contínua da solução com respeito ao dado inicial tem-se $\sup _{t \in\left[T^{\prime}, T^{\prime}\right]}\left\|u^{k}(t)-u(t)\right\|_{H^{1}} \rightarrow 0$, quando $k \rightarrow \infty$, com $T^{\prime}<T$. Logo, $\|u(t)\|_{2}=\left\|u_{0}\right\|_{2} e$ $E(u(t))=E\left(u_{0}\right)$ para todo $t \in[-T, T]$, assim temos (3.15) e (3.14).

A seguir, usando as quantidades conservadas (3.2) e (3.3) veremos três teoremas os quais podem garantir a boa colocação global em $H^{1}(\mathbb{R})$ para (3.1).

Teorema 3.1.2. Se $\lambda>0$ e p $<4$, o problema (3.1) é globalmente bem posto em $H^{1}(\mathbb{R})$.

Demonstração: Queremos mostrar que a norma $H^{1}(\mathbb{R})$ da solução $u(t)$ é uniformemente limitada. Da equação (3.2), obtemos

$$
\left\|u_{x}\right\|_{2}^{2}-2 q|u(0)|^{2}=2 E(u)+\frac{4 \lambda}{p+2}\|u\|_{p+2}^{p+2} .
$$

Da desigualdade de Gagliardo-Nirenberg-Sobolev (Ver [14]), $\|u\|_{p+2}^{p+2} \leq C(p)\left\|u_{x}\right\|_{2}^{\frac{p}{2}}\|u\|_{2}^{\frac{p+4}{2}}$, então, sendo $\lambda>0$,

$$
\left\|u_{x}\right\|_{2}^{2}-2 q|u(0)|^{2} \leq 2 E(u)+4 \lambda C(p)\left\|u_{x}\right\|_{2}^{\frac{p}{2}}\|u\|_{2}^{\frac{p+4}{2}} .
$$

Pela desigualdade de Young com $\varepsilon>0$ (veja [16, Apêndice B.2]) obtemos para $\frac{p}{2}<2$

$$
\left\|u_{x}\right\|_{2}^{\frac{p}{2}}\|u\|_{2}^{\frac{p+4}{2}} \leq \varepsilon\left\|u_{x}\right\|_{2}^{2}+C_{1}(\varepsilon, p)\|u\|_{2}^{\frac{2(p+4)}{4-p}}
$$


onde $C_{1}(\varepsilon, p)=\left(\frac{p}{4 \varepsilon}\right)^{\frac{4-p}{p}}\left(\frac{4-p}{4}\right)$. Pela conservação de energia e de carga, temos então das equações (3.17) e (3.18),

$$
(1-4 \lambda C(p) \varepsilon)\left\|u_{x}\right\|_{2}^{2}-2 q|u(0)|^{2} \leq 2 E\left(u_{0}\right)+4 \lambda C_{1}^{\prime}(p, \varepsilon) Q\left(u_{0}\right)^{\frac{p+4}{4-p}},
$$

sendo $1-4 \lambda C(p) \varepsilon>0$, para $\varepsilon$ pequeno temos

$$
\left\|u_{x}\right\|_{2}^{2}-\frac{2 q|u(0)|^{2}}{1-4 \lambda C(p) \varepsilon} \leq \frac{2 E\left(u_{0}\right)+4 \lambda C_{1}^{\prime}(p, \varepsilon) Q\left(u_{0}\right)^{\frac{p+4}{4-p}}}{1-4 \lambda C(p) \varepsilon} .
$$

Portanto, temos uma limitação uniforme para $\left\|u_{x}\right\|_{2}^{2}$. Agora:

- para $q<0$, temos que $\left\|u_{x}\right\|_{2}^{2} \leq C\left(\left\|u_{0}\right\|_{H^{1}}\right)$. Assim, pela conservação de carga, temos $\|u(t)\|_{H^{1}} \leq C\left(\left\|u_{0}\right\|_{H^{1}}\right)$, para todo $t \in[0, T]$, logo a solução pode ser estendida globalmente;

- para $q>0$, temos similarmente que $\|u(t)\|_{H^{1}} \leq C\left(\left\|u_{0}\right\|_{H^{1}}\right)$.

Isto finaliza a demonstração.

Teorema 3.1.3. Sejam $\lambda>0$ e $p \geq 4$. Suponhamos que existe $\varepsilon>0$ tal que $\left\|u_{0}\right\|_{H^{1}} \ll \varepsilon$, então o problema (3.1) é globalmente bem posto em $H^{1}(\mathbb{R})$.

Demonstração: Da equação (3.17) e pela conservação de energia e carga

$$
\left\|u_{x}\right\|_{2}^{2}-2 q|u(0)|^{2} \leq 2 E\left(u_{0}\right)+4 \lambda C(p)\left\|u_{x}\right\|_{2}^{\frac{p}{2}}\left\|u_{0}\right\|_{2}^{\frac{p+4}{2}} .
$$

Para $p=4$, como $\left\|u_{0}\right\|_{H^{1}} \ll \varepsilon$, temos

$$
\left\|u_{x}\right\|_{2}^{2}-2 q|u(0)|^{2}<2 E\left(u_{0}\right)+4 \lambda C(p) \varepsilon^{4}\left\|u_{x}\right\|_{2}^{2} .
$$

Logo, sendo $1-4 \lambda C(p) \varepsilon^{4}>0$, para $\varepsilon$ pequeno temos

$$
\left\|u_{x}\right\|_{2}^{2}-\frac{2 q|u(0)|^{2}}{1-4 \lambda C(p) \varepsilon^{4}}<\frac{2 E\left(u_{0}\right)}{1-4 \lambda C(p) \varepsilon^{4}} .
$$

Então, temos uma limitação uniforme para $\left\|u_{x}\right\|_{2}^{2}$.

Agora, para $p>4$, temos

$$
\left\|u_{x}\right\|_{2}^{2}-2 q|u(0)|^{2} \leq 2 E\left(u_{0}\right)+4 \lambda C(p) \varepsilon^{\frac{p+4}{2}}\left\|u_{x}\right\|_{2}^{2+\nu},
$$

$\operatorname{com} \nu=\frac{p-4}{2}>0\left(2+\nu=2+\frac{p-4}{2}=\frac{p}{2}>2\right)$. Veja, se $q>0$,

$$
\left\|u_{x}\right\|_{2}^{2} \leq 2 E\left(u_{0}\right)+4 \lambda C(p) \varepsilon^{\frac{p+4}{2}}\left\|u_{x}\right\|_{2}^{2+\nu}+2 q \varepsilon^{2} ;
$$


se $q<0$,

$$
\left\|u_{x}\right\|_{2}^{2} \leq 2 E\left(u_{0}\right)+4 \lambda C(p) \varepsilon^{\frac{p+4}{2}}\left\|u_{x}\right\|_{2}^{2+\nu} .
$$

Logo, como $\left\|u_{0}\right\|_{H^{1}} \ll \varepsilon$, segue de (3.20) e (3.21) avaliado em $t=0$, que $E\left(u_{0}\right)>0$. Defina, $f(t)=\left\|u_{x}\right\|_{2}^{2}$, assim se $q>0, f(t) \leq 2 E\left(u_{0}\right)+4 \lambda C(p) \varepsilon^{\frac{p+4}{2}} f(t)^{\frac{2+\nu}{2}}+2 q \varepsilon^{2}$; se $q<0$, $\left\|u_{x}\right\|_{2}^{2} \leq 2 E\left(u_{0}\right)+4 \lambda C(p) \varepsilon^{\frac{p+4}{2}} f(t)^{\frac{2+\nu}{2}}$. Observe, existe $M_{1}>0$ tal que $f(t)^{\frac{2+\nu}{2}}<M_{1}$, para todo $t \in[0, T]$. Então, existe $M=M\left(\left\|u_{0}\right\|_{H^{1}}, \lambda, p\right)>0$ tal que $\sup _{t \in[0, T]}\left\|u_{x}(t)\right\|_{2}^{2}=\sup _{t \in[0, T]} f(t) \leq M$. Portanto, como $\|u(t)\|_{2}=\|u(0)\|_{2}$, isto permite-nos estender a solução globalmente.

Teorema 3.1.4. Seja $\lambda<0$. O problema (3.1) é globalmente bem posto em $H^{1}(\mathbb{R})$.

Demonstração: Da equação (3.16) e pela conservação de energia, como $\lambda<0$, temos

$$
\left\|u_{x}\right\|_{2}^{2}-2 q\left|u_{0}\right|^{2} \leq 2 E\left(u_{0}\right)
$$

Logo, para $q \neq 0$, temos que $\left\|u_{x}\right\|_{2}^{2}$ é limitada uniformemente, e portanto, $\|u(t)\|_{H^{1}} \leq C\left(\left\|u_{0}\right\|_{H^{1}}\right)$, finalizando a demonstração.

\subsection{Fórmula explícita para o grupo gerado por $H_{q}$ e a projeção espectral contínua}

Nesta seção apresentamos a fórmula explícita para o grupo unitário gerado por $H_{q}$ determinado pelo sistema linear associado com (3.1),

$$
\left\{\begin{array}{c}
i \frac{\partial u}{\partial t}=H_{q} u \\
u(0)=u_{0} .
\end{array}\right.
$$

Esses resultados podem ser encontrados nas referências [5, 12, 15]. Na equação (3.30) abaixo, apresentaremos uma fórmula para a projeção espectral contínua $P_{c}$.

O intuito dessa seção é obter as estimativas para a solução da equação linear associada a NLS- $\delta$.

Usaremos a representação do grupo gerado por $H_{q}$ em termos das autofunções (associadas aos autovalores discretos) e autofunções generalizadas (veja Iorio [28], Holmer et al. [26] e Duchêne et al. [15]). De fato, as famílias de autofunções generalizadas $\left\{\psi_{\lambda}\right\}_{\lambda \in \mathbb{R}}$ são tais que satisfazem

$$
\left\{\begin{array}{l}
H_{q} \psi_{\lambda}=\frac{\lambda^{2}}{2} \psi_{\lambda}, \quad \psi_{\lambda} \text { é contínua } \\
\psi_{\lambda}^{\prime}(0+)-\psi_{\lambda}^{\prime}(0-)=2 q \psi_{\lambda}(0) .
\end{array}\right.
$$

Assim, segundo Holmer et al. [26] e Duchêne et al. [15], obtemos a seguinte família de soluções 
especiais $e_{ \pm}(x, \lambda)$ para $(3.23)$, como a seguinte

$$
e_{ \pm}(x, \lambda)=t_{q}(\lambda) e^{ \pm i \lambda x} \chi_{ \pm}^{0}+\left(e^{ \pm i \lambda x}+r_{q}(\lambda) e^{\mp i \lambda x}\right) \chi_{\mp}^{0},
$$

onde $\chi_{+}^{0}$ é a função característica de $[0,+\infty), \chi_{-}^{0}$ é a função característica de $(-\infty, 0]$ e $t_{q}$ e $r_{q}$ são os coeficientes de transmissão e reflexão

$$
t_{q}(\lambda)=\frac{i \lambda}{i \lambda-q}, \quad r_{q}(\lambda)=\frac{q}{i \lambda-q}
$$

Tais coeficientes satisfazem as seguintes equações

$$
\left|t_{q}(\lambda)\right|^{2}+\left|r_{q}(\lambda)\right|^{2}=1 \quad \text { e } \quad t_{q}(\lambda)=1+r_{q}(\lambda)
$$

A seguir, definindo a família $\left\{\psi_{\lambda}\right\}_{\lambda \in \mathbb{R}}$ como

$$
\psi_{\lambda}(x)=\frac{1}{\sqrt{2 \pi}} \begin{cases}e_{+}(x, \lambda), & \text { para } \lambda \geq 0 \\ e_{-}(x, \lambda), & \text { para } \lambda<0\end{cases}
$$

obtemos do Teorema 2.1.4 as seguintes relações (veja [15], [28]);

1. $\int_{\mathbb{R}} \psi_{0}(x) \overline{\psi_{\lambda}(x)} d x=0$, para todo $\lambda \in \mathbb{R} ;$

2. $\int_{\mathbb{R}} \psi_{\mu}(x) \overline{\psi_{\lambda}(x)} d x=\delta(\lambda-\mu)$, para todo $\mu, \lambda \in \mathbb{R}$

3. $\psi_{0}(x) \psi_{0}(y)+\int_{\mathbb{R}} \psi_{\lambda}(x) \overline{\psi_{\lambda}(y)} d \lambda=\delta(x-y)$, para todo $\lambda \in \mathbb{R}$.

Relembramos que a relação 3. acima, chamada as relações de completamento, no caso $q>0$ é dada por $\int_{\mathbb{R}} \psi_{\lambda}(x) \overline{\psi_{\lambda}(y)} d \lambda=\delta(x-y)$ (a demonstração de 3. para a família $\left\{\psi_{\lambda}\right\}_{\lambda \in \mathbb{R}}$ pode ser provada seguindo as ideias na prova do Lema 3.2.1 abaixo). Mais ainda, a família $\left\{\psi_{\lambda}\right\}_{\lambda \in \mathbb{R}}$ permite-nos definir a transformada de Fourier generalizada

$$
\mathcal{F}(f)(\lambda)=\int_{\mathbb{R}} f(x) \overline{\psi_{\lambda}(x)} d x
$$

e seu adjunto formal $\mathcal{G}(g)(x)=\int_{\mathbb{R}} \psi_{\lambda}(x) g(\lambda) d x$. Assim, de 2 . obtemos imediatamente que $\mathcal{G}$ é a transformada de Fourier inversa, a saber,

$$
f(\lambda)=f * \delta(\lambda)=\int_{\mathbb{R}} \overline{\psi_{\lambda}(x)} \int_{\mathbb{R}} f(\mu) \psi_{\mu}(x) d \mu d x=\mathcal{F}(\mathcal{G g})(\lambda)
$$

Mais ainda, da relação de completamento 3. obtemos para toda $f \in L^{2}(\mathbb{R})$ a seguinte (ortogonal) expansão em autofunções de $H_{q}$,

$$
f=\left\langle f, \psi_{0}\right\rangle \psi_{0}+\int_{\mathbb{R}} \mathcal{F}(f)(\lambda) \psi_{\lambda}(x) d \lambda
$$

Então, para $u \in C\left(\mathbb{R} ; L^{2}(\mathbb{R})\right)$ sendo uma solução de (3.22), o método de separação de variáveis 
implica que para $q<0$

$$
u(x, t)=e^{-i t H_{q}} u_{0}(x)=e^{i \frac{q^{2}}{2} t}\left\langle u_{0}, \psi_{0}\right\rangle \psi_{0}(x)+\int_{\mathbb{R}} e^{i \frac{\lambda^{2}}{2} t} \mathcal{F}\left(u_{0}\right)(\lambda) \psi_{\lambda}(x) d \lambda .
$$

De (3.28), obtemos que a projeção espectral contínua, $P_{c}$, é dada como

$$
P_{c} f(x)=\frac{1}{2 \pi} \int_{\mathbb{R}} \int_{0}^{\infty}\left(e_{+}(x, \lambda) \overline{e_{+}(y, \lambda)}+e_{-}(x, \lambda) \overline{e_{-}(y, \lambda)}\right) f(y) d \lambda d y .
$$

O resultado abaixo descreve explicitamente o propagador $e^{-i t H_{q}}$ em termos do propagador da equação de Schrödinger $e^{i t \Delta}$ (veja Holmer et al. [26], Datchev-Holmer [12]).

Lema 3.2.1. Suponhamos que $\phi \in L^{1}(\mathbb{R})$ e que $\operatorname{supp} \phi \subset(-\infty, 0]$. Então

$$
\begin{aligned}
e^{-i t H_{q}} \phi(x)= & e^{i t \frac{q^{2}}{2}} P_{p} \phi(x)+e^{-i t H_{0}}\left(\phi * \tau_{q}\right)(x) \chi_{+}^{0}(x) \\
& +\left(e^{-i t H_{0}} \phi(x)+e^{-i t H_{0}}\left(\phi * \varrho_{q}\right)(-x)\right) \chi_{-}^{0}(x),
\end{aligned}
$$

onde $H_{0}=-\frac{1}{2} \frac{d^{2}}{d x^{2}}$

$$
\varrho_{q}(x)=q e^{q x} \chi_{+}^{0}(x) \quad e \quad \tau_{q}(x)=\delta(x)+\varrho_{q}(x) .
$$

Demonstração: Chamando $U_{q}(t)=e^{-i t H_{q}}-e^{i t \frac{q^{2}}{2}} P_{p}$, obtemos

$$
\begin{aligned}
U_{q}(t) \phi(x) & =\frac{1}{2 \pi} \int_{0}^{\infty} \int e^{-i t \frac{\lambda^{2}}{2}}\left(e_{+}(x, \lambda) \overline{e_{+}(y, \lambda)}+e_{-}(x, \lambda) \overline{e_{-}(y, \lambda)}\right) \phi(y) d y d \lambda \\
& =\frac{1}{2 \pi} \int_{0}^{\infty} e^{-i t \frac{\lambda^{2}}{2}}\left(e_{+}(x, \lambda) \int \overline{e_{+}(y, \lambda)} \phi(y) d y+e_{-}(x, \lambda) \int \overline{e_{-}(y, \lambda)} \phi(y) d y\right) d \lambda .
\end{aligned}
$$

Mas, como supp $\phi \subset(-\infty, 0]$,

$$
\begin{aligned}
\int \overline{e_{+}(y, \lambda)} \phi(y) d y & =\int_{-\infty}^{0} e^{-i \lambda y} \phi(y) d y+\overline{r_{q}(\lambda)} \int_{\infty}^{0} e^{i \lambda y} \phi(y) d y=\hat{\phi}(\lambda)+r_{q}(-\lambda) \hat{\phi}(-\lambda) \\
\int \overline{e_{-}(y, \lambda)} \phi(y) d y & =\overline{t_{q}(\lambda)} \int_{\infty}^{0} e^{-i \lambda y} \phi(y) d y=t_{q}(-\lambda) \hat{\phi}(-\lambda)
\end{aligned}
$$

Note que

$$
\begin{aligned}
t_{q}(\lambda) r_{q}(-\lambda)+r_{q}(\lambda) t_{q}(-\lambda) & =2 \operatorname{Re}\left(t_{q}(\lambda) \overline{r_{q}(\lambda)}\right)=0, \\
r_{q}(\lambda) r_{q}(-\lambda)+t_{q}(\lambda) t_{q}(-\lambda) & =\left|t_{q}(\lambda)\right|^{2}+\left|r_{q}(\lambda)\right|^{2}=1, \\
\hat{\varrho}_{q}(\lambda) & =q \int_{0}^{\infty} e^{x(q-i \lambda)} d \lambda=r_{q}(\lambda), \\
\hat{\tau}_{q}(\lambda) & =t_{q}(\lambda)
\end{aligned}
$$

Para $x>0$ e usando as equações (3.33) e (3.36), teremos

$$
U_{q}(t) \phi(x)=\frac{1}{2 \pi} \int_{-\infty}^{\infty} e^{-i t \frac{\lambda^{2}}{2}} t_{q}(\lambda) e^{i \lambda x} \hat{\phi}(\lambda) d \lambda=e^{-i t H_{0}}\left(\phi * \tau_{q}\right)(x) .
$$


Por outro lado, para $x<0$, usando as equações (3.34) e (3.35), obtemos

$$
\begin{aligned}
U_{q}(t) \phi(x) & =\frac{1}{2 \pi} \int_{0}^{\infty} e^{-i t \frac{\lambda^{2}}{2}}\left(\hat{\phi}(\lambda) e^{i \lambda x}+r_{q}(\lambda) e^{-i \lambda x} \hat{\phi}(\lambda)\right) d \lambda \\
& =e^{-i t H_{0}} \phi(x)+e^{-i t H_{0}}\left(\phi * \varrho_{q}\right)(-x) .
\end{aligned}
$$

Assim, obtemos (3.31).

Abaixo apresentamos o resultado quando $\phi \in L^{1}(\mathbb{R})$.

Proposição 3.2. Suponhamos que $\phi \in L^{1}(\mathbb{R})$. Então

$$
\begin{aligned}
e^{-i t H_{q}} \phi(x)= & e^{i t \frac{q^{2}}{2}} P_{p} \phi(x)+\left[e^{-i t H_{0}} \phi^{-}(x)+e^{-i t H_{0}}\left(\phi^{-} * \varrho_{q}\right)(-x)\right] \chi_{-}^{0}+e^{-i t H_{0}}\left(\phi^{-} * \tau_{q}\right) \chi_{+}^{0} \\
& +\left[e^{-i t H_{0}} R \phi^{+}(-x)+e^{-i t H_{0}}\left(\phi^{+} * \varrho_{q}\right)(x)\right] \chi_{+}^{0}+e^{-i t H_{0}}\left(R \phi^{+} * R \tau_{q}\right) \chi_{-}^{0}
\end{aligned}
$$

onde $H_{0}=-\frac{1}{2} \frac{d^{2}}{d x^{2}}, R \phi(x)=\phi(-x), \varrho_{q}(x)=q e^{q x} \chi_{[0, \infty)}(x)$ e $\tau_{q}(x)=\delta(x)+\varrho_{q}(x)$.

Demonstração: Seja $\phi \in L^{1}(\mathbb{R})$ e $R \phi(x)=\phi(-x)$. Então, para $\phi^{-}=\phi \chi_{-}^{0}$ e $\phi^{+}=\phi R \chi_{+}^{0}$, temos a decomposição

$$
\phi=\phi^{-}+R \phi^{+} .
$$

Assim, temos, para $x \neq 0$,

$$
H_{q} R \phi(x)=-\frac{1}{2} \phi_{x x}(-x)=R H_{q} \phi(x) .
$$

$\operatorname{supp} \phi^{+} \subset(-\infty, 0]$ e $R(f * R g)=(R f) * g$. Logo, do Lema 3.2.1,

$$
\begin{aligned}
e^{-i t H_{q}} \phi(x)= & e^{i t \frac{q^{2}}{2}} P_{p} \phi(x)+\left[e^{-i t H_{0}} \phi^{-}(x)+e^{-i t H_{0}}\left(\phi^{-} * \varrho_{q}\right)(-x)\right] \chi_{-}^{0}+e^{-i t H_{0}}\left(\phi^{-} * \tau_{q}\right) \chi_{+}^{0} \\
& +\left[e^{-i t H_{0}} R \phi^{+}(x)+e^{-i t H_{0}}\left(R \phi^{+} * \varrho_{q}\right)(-x)\right] \chi_{-}^{0}+e^{-i t H_{0}}\left(R \phi^{+} * \tau_{q}\right) \chi_{+}^{0} \\
= & e^{i t \frac{q^{2}}{2}} P_{p} \phi(x)+\left[e^{-i t H_{0}} \phi^{-}(x)+e^{-i t H_{0}}\left(\phi^{-} * \varrho_{q}\right)(-x)\right] \chi_{-}^{0}+e^{-i t H_{0}}\left(\phi^{-} * \tau_{q}\right) \chi_{+}^{0} \\
& +\left[e^{-i t H_{0}} R \phi^{+}(-x)+e^{-i t H_{0}}\left(\phi^{+} * \varrho_{q}\right)(x)\right] \chi_{+}^{0}+e^{-i t H_{0}}\left(R \phi^{+} * R \tau_{q}\right) \chi_{-}^{0} .
\end{aligned}
$$

Observação 3.2.1. Considere $U_{q}(t)=e^{-i t H_{q}}-e^{i t \frac{q^{2}}{2}} P_{p}$, da Proposição 3.2,

$$
\left\|U_{q} \phi\right\|_{2} \leq\|\phi\|_{2} .
$$




\subsection{Projeções e as componentes pontuais e contínuas associadas à equação (3.1)}

Sejam $P_{c}$ e $P_{p}$ as projeções ortogonais em $L^{2}(\mathbb{R})$ sobre o subespaço espectral contínuo e discreto de $H_{q}, q<0$, respectivamente. Dessa forma, temos que

$$
P_{p}(u)=\operatorname{proj}_{\psi_{0}}(u)=\frac{\left\langle u, \psi_{0}\right\rangle}{\left\langle\psi_{0}, \psi_{0}\right\rangle} \psi_{0}=\left\langle u, \psi_{0}\right\rangle \psi_{0} \equiv u_{p} \psi_{0}
$$

onde $u_{p}=\left\langle u, \psi_{0}\right\rangle \in \mathbb{C}$ e $\psi_{0}(x)=\sqrt{-q} e^{q|x|}$, com $\left\|\psi_{0}\right\|_{2}=1$; ou seja,

$$
P_{p} u(x)=\sqrt{-q} e^{q|x|} \int_{-\infty}^{\infty} \sqrt{-q} e^{q|y|} u(y) d y .
$$

Imediatamente $P_{p}: L^{2}(\mathbb{R}) \rightarrow L^{2}(\mathbb{R})$ e $P_{p}: L^{1}(\mathbb{R}) \rightarrow L^{\infty}(\mathbb{R})$ são operadores contínuos com

$$
\begin{aligned}
\left\|P_{p} u\right\|_{2} & \leq\|u\|_{2}, \quad u \in L^{2}(\mathbb{R}), \\
\left\|P_{p} u\right\|_{\infty} & \leq|q|\|u\|_{1}, \quad u \in L^{1}(\mathbb{R}) .
\end{aligned}
$$

Então pelo Teorema de Riesz-Thorim obtemos $P_{p}: L^{r} \rightarrow L^{r^{\prime}}$, com

$$
\left\|P_{p} u\right\|_{r^{\prime}} \leq(|q|)^{1-\theta}\|u\|_{r}=|q|^{\left(\frac{1}{r}-\frac{1}{r^{\prime}}\right)}\|u\|_{r}
$$

$\operatorname{com} \frac{1}{r}=\frac{\theta}{2}+\frac{1-\theta}{1}$ e $\frac{1}{r^{\prime}}=\frac{\theta}{2}+\frac{1-\theta}{\infty}=\frac{\theta}{2}, \theta \in[0,1] ; \log , \frac{1}{r}+\frac{1}{r^{\prime}}=1$ e $\frac{1}{r}-\frac{1}{r^{\prime}}=1-\theta=1-\frac{2}{r^{\prime}}$.

Assim, para $u=u(x, t)$ solução de (3.1), temos formalmente a decomposição $u(x, t)=$ $u_{p}(t) \psi_{0}(x)+u_{c}(x, t)$, onde $u_{c}(x, t)=P_{c}(u(x, t))$. Note que $\left\langle u_{c}(t), \psi_{0}\right\rangle=0$, para todo $t$, então, $\left\langle\frac{d}{d t} u_{c}(t), \psi_{0}\right\rangle=0$, para todo $t$; ou seja, $\partial_{t} u_{c}(x, t)$ pertence ao espaço contínuo. Aplicando formalmente $P_{p}$ em (3.1), temos

$$
\begin{aligned}
P_{p}\left(i\left(u_{p} \psi_{0}+u_{c}\right)_{t}\right) & =P_{p}\left(H_{q}\left(u_{p} \psi_{0}+u_{c}\right)\right)+P_{p}\left(-\lambda\left|u_{p} \psi_{0}+u_{c}\right|^{p}\left(u_{p} \psi_{0}+u_{c}\right)\right) \\
\Rightarrow\left(i \partial_{t} u_{p}\right) \psi_{0} & =P_{p}\left(H_{q}\left(u_{p} \psi_{0}+u_{c}\right)\right)-\lambda\left\langle\left|u_{p} \psi_{0}+u_{c}\right|^{p}\left(u_{p} \psi_{0}+u_{c}\right), \psi_{0}\right\rangle \psi_{0}
\end{aligned}
$$

mas como $H_{q} \psi_{0}=E_{0} \psi_{0}$, sendo $E_{0}=-\frac{q^{2}}{2}$, temos

$$
\begin{aligned}
P_{p}\left(H_{q}\left(u_{p} \psi_{0}+u_{c}\right)\right) & =\left\langle H_{q}\left(u_{p} \psi_{0}+u_{c}\right), \psi_{0}\right\rangle \psi_{0}=\left\langle H_{q}\left(u_{p} \psi_{0}\right), \psi_{0}\right\rangle \psi_{0}+\left\langle H_{q}\left(u_{c}\right), \psi_{0}\right\rangle \psi_{0} \\
& =E_{0} u_{p} \psi_{0}+\left\langle H_{q}\left(u_{c}\right), \psi_{0}\right\rangle \psi_{0} .
\end{aligned}
$$

Além disso, como $H_{q}$ deixa $\mathscr{R}\left(P_{c}\right)$ invariante, pois $\left\langle H_{q}\left(u_{c}\right), \psi_{0}\right\rangle=\left\langle u_{c}, H_{q}\left(\psi_{0}\right)\right\rangle=\left\langle u_{c}, E_{0} \psi_{0}\right\rangle=$ $E_{0}\left\langle u_{c}, \psi_{0}\right\rangle=0$, então obtemos

$$
\left(i \partial_{t} u_{p}\right) \psi_{0}=E_{0} u_{p} \psi_{0}-\lambda\left\langle\left|u_{p} \psi_{0}+u_{c}\right|^{p}\left(u_{p} \psi_{0}+u_{c}\right), \psi_{0}\right\rangle \psi_{0}
$$

$\operatorname{logo},\left(i \partial_{t} u_{p}-E_{0} u_{p}+\lambda\left\langle\left|u_{p} \psi_{0}+u_{c}\right|^{p}\left(u_{p} \psi_{0}+u_{c}\right), \psi_{0}\right\rangle\right) \psi_{0}=0$, como $\psi_{0} \neq 0$, teremos $i \partial_{t} u_{p}-E_{0} u_{p}+$ 
$\lambda\left\langle\left|u_{p} \psi_{0}+u_{c}\right|^{p}\left(u_{p} \psi_{0}+u_{c}\right), \psi_{0}\right\rangle=0$. Então, se aplicarmos as projeções $P_{c}$ e $P_{p}$ em (3.1) e usamos o fato que $\mathscr{R}\left(P_{p}\right)$ é unidimensional, podemos reescrever (3.1) como o sistema

$$
\left\{\begin{array}{l}
i \partial_{t} u_{p}=E_{0} u_{p}-\lambda f_{p}\left(u_{p}, u_{c}\right), \\
i \partial_{t} u_{c}=H_{q} u_{c}-\lambda f_{c}\left(u_{p}, u_{c}\right),
\end{array}\right.
$$

onde $u_{p}=u_{p}(t) \in \mathbb{C}, u_{c}=u_{c}(t) \in \mathscr{R}\left(P_{c}\right)$, e

$$
\begin{aligned}
& f_{p}\left(u_{p}, u_{c}\right) \equiv\left\langle\left|u_{p} \psi_{0}+u_{c}\right|^{p}\left(u_{p} \psi_{0}+u_{c}\right), \psi_{0}\right\rangle, \\
& f_{c}\left(u_{p}, u_{c}\right) \equiv P_{c}\left(\left|u_{p} \psi_{0}+u_{c}\right|^{p}\left(u_{p} \psi_{0}+u_{c}\right)\right)
\end{aligned}
$$

Se pensarmos em $E^{p}$ (Subespaço gerado pelas autofunções associadas a autovalores com parte real negativa; ou seja, em nosso caso, $E^{p}=\left[\psi_{0}\right]$ ) como o "subespaço central" no Teorema da Variedade Centro Ordinária (veja [23, Teorema 2.9]), podemos procurar uma função $h: E^{p} \rightarrow E^{c} \equiv \mathscr{R}\left(P_{c}\right)$, cujo gráfico é invariante sobre o fluxo gerado por (3.39). Posteriormente, mostraremos que tal função existe (Teorema 4.2.1) e que a "Variedade Centro" definida por seu gráfico é preenchida com órbitas periódicas (Seção 4.1).

\subsection{A equação linear associada a NLS- $\delta$ e estimativas dispersivas}

Considerando a equação linear de Schrödinger

$$
\left\{\begin{array}{l}
i \frac{\partial u}{\partial t}=H_{q} u, \\
u_{0}(x)=u(x, 0) \in H^{1}(\mathbb{R}) .
\end{array}\right.
$$

A partir do que foi discutido na seção anterior, temos o seguinte resultado para $U_{q}(t)=e^{-i t H_{q}}-$ $e^{i t \frac{q^{2}}{2}} P_{p}$.

No Lema 2.2.1 temos uma estimativa para a equação linear associada a NLS. No próximo lema, veremos que também temos a mesma estimativa para a equação linear associada a NLS- $\delta$.

Lema 3.4.1. Se $t \neq 0, \frac{1}{p}+\frac{1}{p^{\prime}}=1$ e $p \in[1,2]$, então $U_{q}(t): L^{p}(\mathbb{R}) \rightarrow L^{p^{\prime}}(\mathbb{R})$ é contínua e

$$
\left\|U_{q}(t) f\right\|_{p^{\prime}} \leq C|t|^{-\left(\frac{1}{p}-\frac{1}{2}\right)}\|f\|_{p}
$$

Mais ainda, se $\sigma>\frac{1}{p}-\frac{1}{2}$, ent $\tilde{a} O$

$$
\left\|U_{q}(t) f\right\|_{L_{-\sigma}^{2}} \leq C|t|^{-\left(\frac{1}{p}-\frac{1}{2}\right)}\|f\|_{p}
$$

Demonstração: Primeiramente, de (3.38), já temos $\left\|U_{q}(t) f\right\|_{2} \leq\|f\|_{2}$. Por outro lado, da Proposição 3.2 e pela desigualdade de Young,

$$
\left\|U_{q} f\right\|_{\infty} \leq \frac{c}{\sqrt{|t|}}\left[\left\|f^{-}\right\|_{1}+\left\|f^{-} * \varrho_{q}\right\|_{1}+\left\|f^{-} * \tau_{q}\right\|_{1}\right]
$$




$$
\begin{aligned}
& +\frac{c}{\sqrt{|t|}}\left[\left\|R f^{+}\right\|_{1}+\left\|f^{+} * \varrho_{q}\right\|_{1}+\left\|R f^{+} * R \tau_{q}\right\|_{1}\right] \\
\leq & \frac{c}{\sqrt{|t|}}\left[3+4\left\|\varrho_{q}\right\|_{1}\right]\|f\|_{1} \\
\leq & \frac{C}{\sqrt{|t|}}\|f\|_{1}
\end{aligned}
$$

Assim, temos $U_{q}(t): L^{2} \rightarrow L^{2}$ e $U_{q}(t): L^{1} \rightarrow L^{\infty}$, então pelo Teorema de Riesz-Thorim obtemos $U_{q}(t): L^{p} \rightarrow L^{p^{\prime}} \operatorname{com} \frac{1}{p}=\frac{\theta}{2}+\frac{1-\theta}{1}$ e $\frac{1}{p^{\prime}}=\frac{\theta}{2}+\frac{1-\theta}{\infty}=\frac{\theta}{2}, \theta \in[0,1], \log \frac{1}{p}+\frac{1}{p^{\prime}}=1 \mathrm{e}$ $\frac{1}{p}-\frac{1}{p^{\prime}}=1-\theta=1-\frac{2}{p^{\prime}}$. Portanto,

$$
\left\|U_{q}(t) f\right\|_{p^{\prime}} \leq\left(C|t|^{-\frac{1}{2}}\right)^{1-\theta}\|f\|_{p}=C|t|^{-\frac{1}{2}\left(\frac{1}{p}-\frac{1}{p^{\prime}}\right)}\|f\|_{p}=C|t|^{-\left(\frac{1}{p}-\frac{1}{2}\right)}\|f\|_{p}
$$

Agora, pela desigualdade de Hölder generalizada e pela desigualdade mostrada, obtemos

$$
\left\|U_{q}(t) f\right\|_{L_{-\sigma}^{2}}=\left\|\langle x\rangle^{-\sigma} U_{q}(t) f\right\|_{2} \leq\left\|\langle x\rangle^{-\sigma}\right\|_{p_{1}}\left\|U_{q}(t) f\right\|_{p} \leq C\left\|\langle x\rangle^{-\sigma}\right\|_{p_{1}}|t|^{-\left(\frac{1}{p}-\frac{1}{2}\right)}\|f\|_{p}
$$

onde $\frac{1}{p_{1}}+\frac{1}{p}=\frac{1}{2}$; isto é, $\frac{1}{p_{1}}=\frac{1}{p}-\frac{1}{2}$. Para que $\left\|\langle x\rangle^{-\sigma}\right\|_{p_{1}}<\infty$, é necessário que $\sigma p_{1}>1$; ou seja, $\sigma>\frac{1}{p_{1}}=\frac{1}{p}-\frac{1}{2}$.

Lema 3.4.2. Se $t \neq 0$, então $\left\|U_{q}(t) f\right\|_{L_{-\sigma}^{2}} \leq C|t|^{-\frac{1}{2}}\|f\|_{L_{\sigma}^{2}}$, para $\sigma>\frac{1}{2}$.

Demonstração: Pelo lema anterior, mostramos que

$$
\left\|U_{q}(t) f\right\|_{L_{-\sigma}^{2}} \leq C|t|^{-\left(\frac{1}{p}-\frac{1}{2}\right)}\|f\|_{p}
$$

$p \in[1,2]$. Em particular, tomando $p=1$ e usando a desigualdade de Cauchy-Schwarz, temos

$$
\begin{aligned}
\left\|U_{q}(t) f\right\|_{L_{-\sigma}^{2}} & \leq C|t|^{-\frac{1}{2}}\|f\|_{1}=C|t|^{-\frac{1}{2}}\left\|\langle x\rangle^{-\sigma}\langle x\rangle^{\sigma} f\right\|_{1} \\
& \leq C|t|^{-\frac{1}{2}}\left\|\langle x\rangle^{-\sigma}\right\|_{2}\left\|\langle x\rangle^{\sigma} f\right\|_{2} \\
& \leq C|t|^{-\frac{1}{2}}\|f\|_{L_{\sigma}^{2}}
\end{aligned}
$$

pois $\left\|\langle x\rangle^{-\sigma}\right\|_{2}<\infty$, para $\sigma>\frac{1}{2}$. 


\section{Caracterização da Variedade Centro}

local $\mathcal{W}_{\mu}^{p}$

Neste capítulo, provaremos que a equação não-linear (3.1) tem uma variedade invariante $W^{p}$ (a variedade centro) que esta próxima de $E^{p}=\left[\psi_{0}\right]$. Em $W^{p}$ todas as órbitas periódicas são da forma

$$
u(x, t)=e^{-i(E t-\theta)} \psi_{E}(x)
$$

onde $\psi_{E}$ é a solução positiva (módulo rotação) de

$$
\left(H_{q}-\lambda\left|\psi_{E}\right|^{p}\right) \psi_{E}=E \psi_{E}
$$

a qual será obtida no Teorema 4.1.1 via o Teorema de Crandall-Rabinowitz. Finalizamos mostrando a existência da variedade centro local no Teorema 4.2 .1 , a saber, $\mathcal{W}_{\mu}^{p}$.

\subsection{Existência de uma curva suave peak-standing waves}

Inicialmente, enfatizamos que $q<0$, pois $\sigma_{p}\left(H_{q}\right)=\left\{-\frac{q^{2}}{2}\right\}$.

Se em (3.1) consideramos $u(x, t)=e^{-i(E t-\theta)} \psi_{E}(x)$, com $\psi_{E} \in \mathscr{D}\left(H_{q}\right)$, temos que $\psi_{E}$ satisfaz formalmente

$$
\left(H_{q}-\lambda\left|\psi_{E}(x)\right|^{p}\right) \psi_{E}(x)=E \psi_{E}(x), \text { para } x \in \mathbb{R}-\{0\} .
$$

O seguinte teorema mostra a existência de uma curva $E \mapsto \psi_{E}$, a qual é solução de (4.1).

Teorema 4.1.1. Seja $E_{0}=-\frac{q^{2}}{2}$. Para $\lambda>0$, seja $E<E_{0}$ e para $\lambda<0$, seja $E \in\left(E_{0}, 0\right)$. Então, para $p>1$, existe uma solução positiva $\psi_{E}(x)$ da equação (4.1), tal que:

(a) $\psi_{E} \in \mathscr{D}\left(H_{q}\right)$;

(b) para $\Omega=\mathbb{R}-\{0\}$, a função $E \mapsto\left\|\psi_{E}\right\|_{H^{2}(\Omega)}$ é diferenciável para $E \neq E_{0}$, e

$$
\lim _{E \rightarrow E_{0}}\left\|\psi_{E}\right\|_{H^{2}(\Omega)}=0
$$


isto é, $\left(\psi_{E}, E\right)$ bifurca a partir da solução zero em $H^{2}(\Omega)$, assim em $L^{p}(\mathbb{R})$, com $p \geq 2^{*}$;

(c) Para todo $\sigma \in \mathbb{R}$, existe uma constante $C_{\sigma}$ tal que

$$
\left\|\langle x\rangle^{\sigma} \psi_{E}\right\|_{H^{2}(\Omega)} \leq C_{\sigma}\left\|\psi_{E}\right\|_{H^{2}(\Omega)}
$$

Demonstração: A ideia é aplicarmos o Teorema de Crandall-Rabinowitz (veja página 20). Consideremos a seguinte aplicação

$$
\begin{aligned}
\mathcal{M}: \mathscr{D}\left(H_{q}\right) \times \mathbb{R} & \longrightarrow L^{2}(\mathbb{R}) \\
(\psi, E) & \longmapsto \mathcal{M}(\psi, E)=H_{q} \psi-\lambda|\psi|^{p} \psi-E \psi
\end{aligned}
$$

Inicialmente note que a aplicação $\psi \mapsto|\psi|^{p} \psi$ é uma aplicação de classe $C^{2}$ para $p>1$. Agora, para $E \in \mathbb{R}$ e $\xi, \phi \in \mathscr{D}\left(H_{q}\right)$, temos

$$
\begin{array}{ll}
\frac{\partial \mathcal{M}}{\partial \psi}(\psi, E)[\xi]=\left(H_{q}-\lambda(p+1)|\psi|^{p}-E\right) \xi ; & \frac{\partial \mathcal{M}}{\partial E}(\psi, E)[1]=-\psi ; \\
\frac{\partial^{2} \mathcal{M}}{\partial E \partial \psi}(\psi, E)[\xi][1]=-\xi ; & \frac{\partial^{2} \mathcal{M}}{\partial \psi \partial E}(\psi, E)[1][\xi]=-\xi ; \\
\frac{\partial^{2} \mathcal{M}}{\partial \psi^{2}}(\psi, E)[\xi][\phi]=-\lambda p(p+1)|\psi|^{p-1} \phi \xi ; & \frac{\partial^{2} \mathcal{M}}{\partial E^{2}}(\psi, E)[1][1]=0 .
\end{array}
$$

Portanto, as derivadas até segunda ordem são contínuas, assim $\mathcal{M}$ é uma aplicação de classe $C^{2}$. Além disso, temos que

- $\mathcal{M}(0, E)=0, \forall E \in \mathbb{R}$

- $\frac{\partial \mathcal{M}}{\partial \psi}\left(0, E_{0}\right)[\xi]=\left(H_{q}-E_{0}\right) \xi$, para todo $\xi \in \mathscr{D}\left(H_{q}\right)$;

- $\mathscr{N}\left(\frac{\partial \mathcal{M}}{\partial \psi}\left(0, E_{0}\right)\right)=\left[\psi_{0}\right], \operatorname{com}\left\|\psi_{0}\right\|_{2}=1$;

onde $\psi_{0}>0$ é dada explicitamente pela equação $(2.24)$. Considere $\mathcal{L}_{0}=\frac{\partial \mathcal{M}}{\partial \psi}\left(0, E_{0}\right)$. Como $\mathcal{L}_{0}$ é autoadjunto, $\operatorname{dim}\left(\mathscr{N}\left(\mathcal{L}_{0}\right)\right)=\operatorname{codim}\left(\mathscr{R}\left(\mathcal{L}_{0}\right)\right)=1$.

Agora, suponhamos que

$$
\frac{\partial^{2} \mathcal{M}}{\partial E \partial \psi}\left(0, E_{0}\right) \psi_{0}=-\psi_{0} \in \mathscr{R}\left(\mathcal{L}_{0}\right)=\left[\mathscr{N}\left(\mathcal{L}_{0}\right)\right]^{\perp}
$$

logo, $\int\left|\psi_{0}(x)\right|^{2} d x=0$, um absurdo, pois $\left\|\psi_{0}\right\|_{2}=1$; então $-\psi_{0} \notin \mathscr{R}\left(\mathcal{L}_{0}\right)$. Portanto, pelo Teorema de Crandall-Rabinowitz, Teorema 2.3.2, $\left(0, E_{0}\right)$ é um ponto de bifurcação; ou seja, existe uma curva $\left(E, \psi_{E}\right)$, tal que $\mathcal{M}\left(E, \psi_{E}\right)=0$ para $E$ em uma vizinhança de $E_{0}$. Assim $\psi_{E} \in \mathscr{D}\left(H_{q}\right)$ e satisfaz

$$
\left(H_{q}-\lambda\left|\psi_{E}\right|^{p}\right) \psi_{E}=E \psi_{E} .
$$

\footnotetext{
${ }^{*}$ Imersão de Sobolev: $H^{2}(\Omega) \subset L^{p}(\mathbb{R})$
} 
Agora para $\psi_{0}$, temos $\left\langle\left(H_{q}-\lambda\left|\psi_{E}\right|^{p}\right) \psi_{E}, \psi_{0}\right\rangle=\left\langle E \psi_{E}, \psi_{0}\right\rangle$, como $H_{q}$ é autoadjunto e $H_{q} \psi_{0}=E_{0} \psi_{0}$, obtemos

$$
\left(E_{0}-E\right)\left\langle\psi_{E}, \psi_{0}\right\rangle=\lambda\left\langle\left|\psi_{E}\right|^{p} \psi_{E}, \psi_{0}\right\rangle
$$

Sendo assim, desde que $\psi_{E}>0$ (Pela unicidade de solução em [17, 18, 29]), então

- se $\lambda>0$, então $E<E_{0}$ (veja $\left.[17,18]\right)$;

- se $\lambda<0$, então $E>E_{0}$, ou seja, $E \in\left(E_{0}, 0\right)$ (veja [29]).

Para provarmos o item $(c)$ basta lembrar que pela unicidade de solução como comentamos na introdução desse trabalho, obtemos

$$
\psi_{E}(x, t)=\left[\sqrt{\frac{p+2}{4 \lambda}} \sqrt{-2 E} \operatorname{sech}\left(\frac{p}{2} \sqrt{-2 E}|x|+\tanh ^{-1}\left(-\frac{q}{\sqrt{-2 E}}\right)\right)\right]^{\frac{2}{p}},
$$

se $E<E_{0}$ e $\lambda>0$ e

$$
\psi_{E}(x, t)=\left[\sqrt{\frac{p+2}{4|\lambda|}} \sqrt{-2 E} \operatorname{cossech}\left(\frac{p}{2} \sqrt{-2 E}|x|+\tanh ^{-1}\left(\frac{\sqrt{-2 E}}{|q|}\right)\right)\right]^{\frac{2}{p}},
$$

se $E_{0}<E<0$ e $\lambda<0$.

\subsection{Caracterização de $\mathcal{W}_{\mu}^{p}$}

O objetivo principal dessa seção é mostrar a existência da variedade centro local e sua caracterização. A variedade invariante irá consistir de uma família de órbitas periódicas da forma $e^{-i(E t-\theta)} \psi_{E}(x)$, para $E \in I \subseteq \mathbb{R}$, assim definimos o conjunto

$$
\mathcal{W}_{\mu}^{p}=\left\{e^{i \theta} \psi_{E}(x):\left|E-E_{0}\right|<\mu \text { e } 0 \leq \theta \leq 2 \pi\right\}
$$

$\mathcal{W}_{\mu}^{p}$ será a "variedade centro local" em nossa interpretação. A ideia principal é escrever $\mathcal{W}_{\mu}^{p}$ como o gráfico de uma função do subespaço linear gerado por $\psi_{0}$, em seu complemento $\mathscr{R}\left(P_{c}\right)$. A saber, dado um ponto $e^{i \theta} \psi_{E} \in \mathcal{W}_{\mu}^{p}$, o escreveremos da seguinte forma

$$
e^{i \theta} \psi_{E}(x)=u_{p} \psi_{0}+h\left(u_{p}\right)
$$

onde $u_{p}=\left\langle e^{i \theta} \psi_{E}, \psi_{0}\right\rangle \in \mathbb{C}$ e $h\left(u_{p}\right) \in \mathscr{R}\left(P_{c}\right)$. Assim, vamos provar a seguinte teorema:

Teorema 4.2.1. Existem um intervalo ao redor de $E_{0},\left(E_{0}-\mu, E_{0}+\mu\right)$, para $\mu$ pequeno, um $\delta>0$ e uma função $h$ de classe $C^{1}$

$$
h:\left\{u_{p} \in \mathbb{C}:\left|u_{p}\right|<\delta\right\} \rightarrow L_{\sigma}^{2}(\mathbb{R}) \cap H^{1}(\mathbb{R}) \cap \mathscr{R}\left(P_{c}\right),
$$


tal que a variedade centro local $\mathcal{W}_{\mu}^{p}$ é dada por

$$
\mathcal{W}_{\mu}^{p}=\left\{\psi: \psi=u_{p} \psi_{0}+h\left(u_{p}\right) ;\left|u_{p}\right|<\delta\right\} .
$$

Notamos que $h(0)=0$. Além disso, $h$ e $E=E\left(\left|u_{p}\right|\right)$ satisfazem o sistema

$$
\left\{\begin{aligned}
h\left(u_{p}\right) & =\lambda\left(H_{q}-E\right)^{-1} f_{c}\left(u_{p}, h\left(u_{p}\right)\right), \\
E_{0}-E & =\lambda u_{p}^{-1} f_{p}\left(u_{p}, h\left(u_{p}\right)\right),
\end{aligned}\right.
$$

$e \operatorname{assim} h\left(u_{p}\right) \in \mathscr{R}\left(P_{c}\right)$.

Antes de iniciar a demonstração do Teorema 4.2.1, iremos demonstrar alguns resultados que nos serão úteis.

Lema 4.2.1. Seja $\alpha>0$. Então, o operador

$$
\langle x\rangle^{\alpha}\left(H_{0}-E\right)^{-1}\langle x\rangle^{-\alpha} \quad: \quad L^{2}(\mathbb{R}) \rightarrow L^{2}(\mathbb{R})
$$

é limitado para $E \in \rho\left(H_{0}\right)=\rho\left(-\Delta_{q}\right)=\mathbb{C}-[0, \infty)$ e $\operatorname{Im}(E)>0$.

Demonstração: Primeiramente, para $f \in L_{\alpha}^{2}(\mathbb{R})$ e $\alpha>0$,

$$
\|f\|_{2}^{2}=\int_{\mathbb{R}}|f(x)|^{2} d x \leq \int_{\mathbb{R}}\left|\langle x\rangle^{\alpha} f(x)\right|^{2} d x=\|f\|_{L_{\alpha}^{2}}^{2},
$$

ou seja, $L_{\alpha}^{2}(\mathbb{R}) \subset L^{2}(\mathbb{R})$.

Consideremos $k=\sqrt{-E} i$. Então $\operatorname{Im} k>0$. Seja $G_{k}(x)=\frac{i}{2 k} e^{i k|x|}$. Assim, pelo Teorema 2.1.2, para $h \in L^{2}(\mathbb{R})$,

$$
\left(H_{0}-E\right)^{-1} h=G_{k} * h .
$$

Seja $g \in L^{2}(\mathbb{R})$, então

$$
\begin{aligned}
\left\|\langle x\rangle^{\alpha}\left(H_{0}-E\right)^{-1}\langle x\rangle^{-\alpha} g\right\|_{2}^{2} & =\int_{\mathbb{R}}\left|\langle x\rangle^{\alpha}\left(G_{k} *\langle y\rangle^{-\alpha} g\right)(x)\right|^{2} d x \\
& =\int_{\mathbb{R}}\left|\int_{\mathbb{R}} G_{k}(x-y)\langle x\rangle^{\alpha}\langle y\rangle^{-\alpha} g(y) d y\right|^{2} d x,
\end{aligned}
$$

mas, como para todo $x, y \in \mathbb{R}$,

$$
\langle x\rangle^{\alpha}\langle y\rangle^{-\alpha} \leq C\left(1+\langle x-y\rangle^{\alpha}\right)
$$

e $G_{k},\langle x\rangle^{\alpha} G_{k} \in L^{1}(\mathbb{R})$, temos

$$
\begin{aligned}
& \left\|\langle x\rangle^{\alpha}\left(H_{0}-E\right)^{-1}\langle x\rangle^{-\alpha} g\right\|_{2}^{2} \\
\leq & 2 C \int_{\mathbb{R}}\left|\int_{\mathbb{R}} G_{k}(x-y) g(y) d y\right|^{2} d x+2 C \int_{\mathbb{R}}\left|\int_{\mathbb{R}} G_{k}(x-y)\langle x-y\rangle^{\alpha} g(y) d y\right|^{2} d x \\
= & 2 C\left(\left\|G_{k} * g\right\|_{2}^{2}+\left\|G_{k}\langle\cdot\rangle^{\alpha} * g\right\|_{2}^{2}\right) \leq 2 C\left(\left\|G_{k}\right\|_{1}^{2}\|g\|_{2}^{2}+\left\|G_{k}\langle\cdot\rangle^{\alpha}\right\|_{1}^{2}\|g\|_{2}^{2}\right)
\end{aligned}
$$




$$
=C_{1}\|g\|_{2}^{2}
$$

o qual finaliza a demonstração.

Agora, ao substituirmos formalmente $e^{i \theta} \psi_{E}=u_{p} \psi_{0}+h\left(u_{p}\right)$, com $u_{p} \in \mathbb{C}$ e $h\left(u_{p}\right) \in \mathscr{R}\left(P_{c}\right)$, em (4.1) teremos

$$
\left(H_{q}-\lambda\left|u_{p} \psi_{0}+h\left(u_{p}\right)\right|^{p}\right)\left(u_{p} \psi_{0}+h\left(u_{p}\right)\right)=E\left(u_{p} \psi_{0}+h\left(u_{p}\right)\right) .
$$

Aplicando $P_{c}$ e $P_{p}$ em (4.4), respectivamente, obtemos que a procurada $h$ tem que satisfazer o sistema $^{\dagger}$

$$
\left\{\begin{aligned}
h\left(u_{p}\right) & =\lambda\left(H_{q}-E\right)^{-1} f_{c}\left(u_{p}, h\left(u_{p}\right)\right), \\
E_{0}-E & =\lambda u_{p}^{-1} f_{p}\left(u_{p}, h\left(u_{p}\right)\right),
\end{aligned}\right.
$$

com $u_{p} \neq 0$, onde as funções $f_{c}$ e $f_{p}$ são dadas pela equação (3.40). Notamos que, obviamente $h\left(u_{p}\right) \in \mathscr{R}\left(P_{c}\right)$ e para todo $\theta \in \mathbb{R}, u_{p} \in \mathbb{C}$ e $u_{c} \in \mathscr{R}\left(P_{c}\right)$, temos as relações

$$
\begin{aligned}
& f_{c}\left(e^{i \theta} u_{p}, e^{i \theta} u_{c}\right)=e^{i \theta} f_{c}\left(u_{p}, u_{c}\right), \\
& f_{p}\left(e^{i \theta} u_{p}, e^{i \theta} u_{c}\right)=e^{i \theta} f_{p}\left(u_{p}, u_{c}\right) .
\end{aligned}
$$

Portanto, para $u_{p}=\left|u_{p}\right| e^{i \theta}$ (forma polar), obtemos a relação

$$
h\left(u_{p}\right)=h\left(e^{i \theta} u_{p} e^{-i \theta}\right)=e^{i \theta} h\left(u_{p} e^{-i \theta}\right)=\frac{u_{p}}{\left|u_{p}\right|} h\left(\left|u_{p}\right|\right) .
$$

Logo, da relação anterior, basta-nos considerarmos $h$ como uma função real de uma variável $r$, $h=h(r)$.

A seguir vamos determinar a função $h$. Iniciamos com o seguinte resultado de regularidade.

Lema 4.2.2. Consideremos $I_{1}=\left(E_{0}-\delta, E_{0}+\delta\right)$ e $I_{2}=(-\delta, \delta)$, sendo $\delta$ suficientemente pequeno. A aplicação

$$
\begin{aligned}
\mathscr{K}: I_{1} \times I_{2} \times\left(L_{\sigma}^{2} \cap H^{1}\right) & \rightarrow L_{\sigma}^{2} \cap H^{1} \\
(E, r, h) & \mapsto \mathscr{K}(E, r, h)=h-\lambda\left(H_{q}-E\right)^{-1} f_{c}(r, h)
\end{aligned}
$$

é de classe $C^{1}$, para $\sigma>\frac{1}{2}$.

Demonstração: Primeiramente, veremos que $\mathscr{K}$ está bem definida em $E_{0}$. Sabemos que $E_{0}$ é um ponto isolado de $\sigma\left(H_{q}\right)$ e $H_{q}$ é autoadjunto. Então, por [25, Teorema 6.7], $H_{q}-E_{0}$ restrito ao $\mathscr{D}\left(H_{q}\right) \cap\left[\mathscr{N}\left(H_{q}-E_{0}\right)\right]^{\perp}=\mathscr{D}\left(H_{q}\right) \cap \mathscr{R}\left(P_{c}\right)=\mathfrak{M}$ tem inverso limitado; isto é, $E_{0} \notin \sigma\left(\left.H_{q}\right|_{\mathfrak{M}}\right)$. Logo, $\mathscr{K}$ está bem definida em $E_{0}$, pois $f_{c}(r, h) \in \mathscr{R}\left(P_{c}\right)$.

\footnotetext{
${ }^{\dagger}$ Lembre que independente do sinal de $\lambda$ e da estrutura do espectro de $H_{q}$, o valor de $E$ sempre estará na resolvente do operador $H_{q}$
} 
A seguir, mostraremos que $\mathscr{K}(E, r, h) \in L_{\sigma}^{2} \cap H^{1}$. Observe

$$
\begin{aligned}
\|\mathscr{K}(E, r, h)\|_{L_{\sigma}^{2}}^{2} & =\int_{\mathbb{R}}\left|\left(h(x)-\lambda\left(H_{q}-E\right)^{-1} f_{c}(r, h(x))\right)\langle x\rangle^{\sigma}\right|^{2} d x \\
& \leq 2\|h\|_{L_{\sigma}^{2}}^{2}+2|\lambda| \int_{\mathbb{R}}\left|\langle x\rangle^{\sigma}\left(\left(H_{q}-E\right)^{-1} f_{c}(r, h(x))\right)\right|^{2} d x .
\end{aligned}
$$

Agora da equação (2.17), temos que

$$
\left(H_{q}-E\right)^{-1} f_{c}(r, h(x))=\left(H_{0}-E\right)^{-1} f_{c}(r, h(x))+\int_{\mathbb{R}} \frac{q}{2 k(i q+2 k)} e^{i k(|y|+|x|)} f_{c}(r, h(y)) d y,
$$

onde $k=\sqrt{-E} i$, então

$$
\begin{aligned}
& \left|\langle x\rangle^{\sigma}\left(\left(H_{q}-E\right)^{-1} f_{c}(r, h(x))\right)\right|^{2} \\
\leq & 2\left|\langle x\rangle^{\sigma}\left(H_{0}-E\right)^{-1} f_{c}(r, h(x))\right|^{2}+\frac{C_{q, E} e^{-2 \sqrt{-E}|x|}\langle x\rangle^{2 \sigma}}{\sqrt{-E}}\left(\int_{\mathbb{R}} e^{-\sqrt{-E}|y|}\left|f_{c}(r, h(y))\right| d y\right)^{2},
\end{aligned}
$$

onde $C_{q, E}=\left|\frac{q}{i q+2 k}\right|^{2}$ e lembrando que $f_{c}(r, h(x))=P_{c}\left(\left|r \psi_{0}(x)+h(x)\right|^{p}\left(r \psi_{0}(x)+h(x)\right)\right)$. A seguir, note que para $f \in L^{2}$, podemos escrever, $f=P_{c} f+P_{p} f$, logo $\langle x\rangle^{\sigma} P_{c} f=\langle x\rangle^{\sigma} f-\langle x\rangle^{\sigma} P_{p} f$. Então,

$$
\left\|\langle x\rangle^{\sigma} P_{c} f\right\|_{2} \leq\|f\|_{L_{\sigma}^{2}}+\left|\left\langle f, \psi_{0}\right\rangle\right|\left\|\langle x\rangle^{\sigma} \psi_{0}\right\|_{2} \leq\|f\|_{L_{\sigma}^{2}}+c\|f\|_{2}
$$

Chamando $f:=\left|r \psi_{0}+h\right|^{p}\left(r \psi_{0}+h\right)$, como $H^{1} \hookrightarrow L^{\infty}$, temos

$$
\|f\|_{2}^{2}=\int_{\mathbb{R}}\left|r \psi_{0}(x)+h(x)\right|^{2 p}\left|r \psi_{0}(x)+h(x)\right|^{2} d x \leq\left\|r \psi_{0}+h\right\|_{\infty}^{2 p}\left\|r \psi_{0}+h\right\|_{2}^{2} \leq c_{1}\left\|r \psi_{0}+h\right\|_{H^{1}}^{2 p+2}
$$

e

$$
\|f\|_{L_{\sigma}^{2}}=\left\|\langle x\rangle^{\sigma}\left|r \psi_{0}+h\right|^{p}\left(r \psi_{0}+h\right)\right\|_{2}^{2} \leq\left\|r \psi_{0}+h\right\|_{\infty}^{2 p}\left\|\langle x\rangle^{\sigma}\left(r \psi_{0}+h\right)\right\|_{2}^{2} \leq c_{1}\left\|r \psi_{0}+h\right\|_{H^{1} \cap L_{\sigma}^{2}}^{2 p+2} .
$$

Agora, para a primeira parcela de (4.8), temos Lema 4.2.1, de (4.9) e das duas estimativas acima, obtemos

$$
\left\|\langle x\rangle^{\sigma}\left(H_{0}-E\right)^{-1} f_{c}(r, h)\right\|_{2}^{2} \leq c\left\|r \psi_{0}+h\right\|_{H^{1} \cap L_{\sigma}^{2}}^{2 p+2}
$$

por outro lado,

$$
\begin{aligned}
& \int_{\mathbb{R}} e^{-2 \sqrt{-E}|x|}\langle x\rangle^{2 \sigma}\left(\int_{\mathbb{R}} e^{-\sqrt{-E}|y|}\left|f_{c}(r, h(y))\right| d y\right)^{2} d x \\
= & \int_{\mathbb{R}} e^{-2 \sqrt{-E}|x|}\langle x\rangle^{2 \sigma} d x\left(\int_{\mathbb{R}} e^{-\sqrt{-E}|y|}\left|f_{c}(r, h(y))\right| d y\right)^{2} \\
\leq & C_{2}\left(\int_{\mathbb{R}} e^{-2 \sqrt{-E}|y|} d y\right)\left(\int_{\mathbb{R}}\left|f_{c}(r, h(y))\right|^{2} d y\right)=C_{2} C_{3}\left\|P_{c}\left(\left|r \psi_{0}+h\right|^{p}\left(r \psi_{0}+h\right)\right)\right\|_{2}^{2} \\
\leq & C_{2} C_{3}\left\|\left|r \psi_{0}+h\right|^{p}\left(r \psi_{0}+h\right)\right\|_{2}^{2} \leq C_{1} C_{2} C_{3}\left\|r \psi_{0}+h\right\|_{H^{1}}^{2 p}\left\|r \psi_{0}+h\right\|_{2}^{2} \\
\leq & C_{1} C_{2} C_{3}\left\|r \psi_{0}+h\right\|_{H^{1}}^{2 p+2}
\end{aligned}
$$


com, $C_{2}=\int_{\mathbb{R}} e^{-2 \sqrt{-E}|x|}\langle x\rangle^{2 \sigma} d x<\infty$ e $C_{3}=\int_{\mathbb{R}} e^{-2 \sqrt{-E}|y|} d y<\infty$. Logo,

$$
\|\mathscr{K}(E, r, h)\|_{\sigma}^{2} \leq 2\|h\|_{L_{\sigma}^{2}}^{2}+2 c\left\|r \psi_{0}+h\right\|_{H^{1} \cap L_{\sigma}^{2}}^{2 p+2}+2 C_{4}\left\|r \psi_{0}+h\right\|_{H^{1}}^{2 p+2} \leq C\|h\|_{L_{\sigma}^{2} \cap H^{1}}^{2 p+2}<\infty .
$$

Então, $\mathscr{K}(E, r, h) \in L_{\sigma}^{2}(\mathbb{R})$. Observe que $\mathscr{K}(E, r, h) \in H^{1}(\mathbb{R})$, pois do Teorema 2.1.2, temos que $\left(H_{q}-E\right)^{-1} f_{c}(r, h(x)) \in \mathscr{D}\left(H_{q}\right) \subset H^{1}(\mathbb{R})$ e $h \in H^{1}$. Portanto, $\mathscr{K}(E, r, h) \in L_{\sigma}^{2}(\mathbb{R}) \cap H^{1}(\mathbb{R})$.

Agora mostraremos que $\mathscr{K}$ é de classe $C^{1}$. Note que $f_{c}(r, h)=P_{c} G\left(r \psi_{0}+h\right)$, onde a aplicação $G$ é dada por

$$
\begin{aligned}
G: H^{1}(\mathbb{R}) & \longrightarrow H^{1}(\mathbb{R}) \\
\varphi & \longmapsto G(\varphi)=|\varphi|^{p} \varphi .
\end{aligned}
$$

Como, $G^{\prime}(\varphi)=(p+1)|\varphi|^{p}$. Claramente, $G \in C^{1}\left(H^{1}(\mathbb{R}), H^{1}(\mathbb{R})\right)$. Mais ainda, facilmente, mostra-se que $G \in C^{1}\left(H^{1}(\mathbb{R}) \cap L_{\sigma}^{2}(\mathbb{R}), L_{\sigma}^{2}(\mathbb{R})\right)$.

A seguir provaremos $\left\|\langle x\rangle^{\sigma}\left(H_{q}-E\right)^{-1} P_{c} G(\varphi)\right\|_{2}^{2} \leq c\|\varphi\|_{H^{1}}^{2 p}\|\varphi\|_{L_{\sigma}^{2}}^{2}$, para $\varphi \in H^{1} \cap L_{\sigma}^{2}$. De fato, da equação (2.17), temos

$$
\left(H_{q}-E\right)^{-1} P_{c} G(\varphi)(x)=\left(H_{0}-E\right)^{-1} P_{c} G(\varphi)(x)+\int_{\mathbb{R}} \frac{q}{2 k(i q+2 k)} e^{i k(|y|+|x|)} P_{c} G(\varphi)(y) d y,
$$

onde $k=\sqrt{-E} i$. Lembramos que

$$
\left(H_{0}-E\right)^{-1} P_{c} G(\varphi)(x)=\left(G_{k} * P_{c} G(\varphi)\right)(x)=\int_{\mathbb{R}} \frac{i}{2 k} e^{i k|x-y|} P_{c} G(\varphi)(y) d y
$$

e, como $P_{c} f=f-P_{p} f=f-\left\langle f, \psi_{0}\right\rangle \psi_{0}$, temos

$$
e^{i k|x|} \int_{\mathbb{R}} e^{i k|y|} P_{c}\left(|\varphi|^{p} \varphi\right)(y) d y=e^{i k|x|} \int_{\mathbb{R}} e^{i k|y|}|\varphi(y)|^{p} \varphi(y) d y-e^{i k|x|} \int_{\mathbb{R}} e^{i k|y|} P_{p}\left(|\varphi|^{p} \varphi\right)(y) d y,
$$

então

$$
\begin{aligned}
\left(H_{q}-E\right)^{-1} P_{c} G(\varphi)(x)= & \left(G_{k} * P_{c} G(\varphi)\right)(x)+\frac{q}{2 k(i q+2 k)} e^{i k|x|} \int_{\mathbb{R}} e^{i k|y|}|\varphi(y)|^{p} \varphi(y) d y \\
& -\frac{q}{2 k(i q+2 k)} e^{i k|x|} \int_{\mathbb{R}} e^{i k|y|}\left\langle|\varphi|^{p} \varphi, \psi_{0}\right\rangle \psi_{0}(y) d y .
\end{aligned}
$$

Agora, usando o Lema 4.2.1, obtemos

$$
\begin{aligned}
& \left\|\langle x\rangle^{\sigma}\left(H_{0}-E\right)^{-1} P_{c}\left(|\varphi|^{p} \varphi\right)\right\|_{2}^{2}=\left\|\langle x\rangle^{\sigma}\left(H_{0}-E\right)^{-1}\langle x\rangle^{-\sigma}\langle x\rangle^{\sigma} P_{c}\left(|\varphi|^{p} \varphi\right)\right\|_{2}^{2} \\
\leq & C\left\|\langle x\rangle^{\sigma} P_{c}\left(|\varphi|^{p} \varphi\right)\right\|_{2}^{2} \leq C\left\|\langle x\rangle^{\sigma}|\varphi|^{p} \varphi\right\|_{2}^{2}+C\left\|\langle x\rangle^{\sigma}\left\langle|\varphi|^{p} \varphi, \psi_{0}\right\rangle \psi_{0}\right\|_{2}^{2} \\
\leq & C\|\varphi\|_{H^{1}}^{2 p}\|\varphi\|_{L_{\sigma}^{2}}^{2}+C\left|\left\langle|\varphi|^{p} \varphi, \psi_{0}\right\rangle\right|^{2}\left\|\psi_{0}\right\|_{L_{\sigma}^{2}}^{2} \leq C\|\varphi\|_{H^{1}}^{2 p}\|\varphi\|_{L_{\sigma}^{2}}^{2}+C\|\varphi\|_{H^{1}}^{2 p}\|\varphi\|_{2}^{2}\left\|\psi_{0}\right\|_{L_{\sigma}^{2}}^{2} \\
\leq & C\|\varphi\|_{H^{1}}^{2 p}\|\varphi\|_{L_{\sigma}^{2}}^{2}
\end{aligned}
$$

e lembrando que $i k=-\sqrt{-E}$ concluímos as seguintes estimativas,

$$
\left.\left.\int_{\mathbb{R}}\left|\langle x\rangle^{\sigma} e^{i k|x|} \int_{\mathbb{R}} e^{i k|y|}\right| \varphi(y)\right|^{p} \varphi(y) d y\right|^{2} d x=\left.\left.\int_{\mathbb{R}}\left|\langle x\rangle^{\sigma} e^{i k|x|}\right|^{2} d x\left|\int_{\mathbb{R}} e^{i k|y|}\right| \varphi(y)\right|^{p} \varphi(y) d y\right|^{2}
$$




$$
\begin{aligned}
& \leq C\|\varphi\|_{H^{1}}^{2 p}\left(\int_{\mathbb{R}}\left|\langle x\rangle^{\sigma} e^{i k|x|}\right|^{2} d x\right)\left|\int_{\mathbb{R}} e^{i k|y|} \varphi(y) d y\right|^{2} \leq C\|\varphi\|_{H^{1}}^{2 p}\|\varphi\|_{L_{\sigma}^{2}}^{2}, \\
& \int_{\mathbb{R}}\left|\langle x\rangle^{\sigma} e^{i k|x|} \int_{\mathbb{R}} e^{i k|y|}\left\langle|\varphi|^{p} \varphi, \psi_{0}\right\rangle \psi_{0}(y) d y\right|^{2} d x=C\left|\left\langle|\varphi|^{p} \varphi, \psi_{0}\right\rangle\right|^{2}\left|\int_{\mathbb{R}} e^{i k|y|} \psi_{0}(y) d y\right|^{2} \\
& \leq C\|\varphi\|_{H^{1}}^{2 p}\|\varphi\|_{L_{\sigma}^{2}}^{2},
\end{aligned}
$$

sendo $C$ é uma constante positiva. Logo,

$$
\left\|\langle x\rangle^{\sigma}\left(H_{q}-E\right)^{-1} P_{c} G(\varphi)\right\|_{2}^{2} \leq c\|\varphi\|_{H^{1}}^{2 p}\|\varphi\|_{L_{\sigma}^{2}}^{2} .
$$

Então,

$$
\left\|\langle x\rangle^{\sigma}\left(H_{q}-E\right)^{-1} P_{c} G(\varphi)\right\|_{2} \leq c\|\varphi\|_{H^{1} \cap L_{\sigma}^{2}}^{p+1} .
$$

Mostraremos agora que $\left(H_{q}-E\right)^{-1} P_{c} G(\varphi) \in C^{1}\left(I_{1} \times H^{1}(\mathbb{R}), H^{1}(\mathbb{R})\right)$. Claramente,

$$
\left(H_{q}-E\right)^{-1} P_{c} G(\varphi) \in H^{1}(\mathbb{R}) .
$$

Pelo Teorema 2.1.2, temos

$$
\left(H_{q}-E\right)^{-1}=\left(H_{0}-E\right)^{-1}-\frac{2 q k}{i q+2 k}\left\langle\cdot, \overline{G_{k}(\cdot)}\right\rangle G_{k}(\cdot) .
$$

Logo, chamando $\mathcal{A}=\left(H_{q}-E\right)^{-1}$ e recordando que $\left(H_{0}-E\right)^{-1} P_{c} G(\varphi)=\left(G_{k} * P_{c} G(\varphi)\right)$ e $k=\sqrt{-E} i$, das relações

$$
\begin{aligned}
\mathcal{A} P_{c} G(\varphi)= & \left(H_{0}-E\right)^{-1} P_{c} G(\varphi)-\frac{2 q k}{i q+2 k}\left\langle P_{c} G(\varphi), \overline{G_{k}(\cdot)}\right\rangle G_{k}(\cdot) \\
\frac{\partial \mathcal{A} P_{c} G(\varphi)}{\partial \varphi}= & \left(H_{0}-E\right)^{-1} P_{c} G^{\prime}(\varphi)-\frac{2 q k}{i q+2 k}\left\langle P_{c} G^{\prime}(\varphi), \overline{G_{k}(\cdot)}\right\rangle G_{k}(\cdot), \\
\frac{\partial \mathcal{A} P_{c} G(\varphi)}{\partial E}= & \left(\frac{\partial G_{k}}{\partial E} * P_{c} G(\varphi)\right)-\frac{\partial}{\partial E}\left(\frac{2 q k}{i q+2 k}\right)\left\langle P_{c} G(\varphi), \overline{G_{k}(\cdot)}\right\rangle G_{k}(\cdot) \\
& -\frac{2 q k}{i q+2 k}\left(\left\langle P_{c} G(\varphi), \frac{\partial \overline{G_{k}(\cdot)}}{\partial E}\right\rangle G_{k}(\cdot)+\left\langle P_{c} G(\varphi), \overline{G_{k}(\cdot)}\right\rangle \frac{\partial G_{k}(\cdot)}{\partial E}\right),
\end{aligned}
$$

vemos imediatamente que são elas contínuas em $I_{1} \times H^{1}(\mathbb{R})$. Então, das relações (4.11), (4.12), (4.13) e (4.14), temos $\left(H_{q}-E\right)^{-1} P_{c} G(\varphi) \in C^{1}\left(I_{1} \times H^{1}(\mathbb{R}) \cap L_{\sigma}^{2}(\mathbb{R}), H^{1}(\mathbb{R}) \cap L_{\sigma}^{2}(\mathbb{R})\right)$. Logo, $\mathscr{K}(E, r, h) \in C^{1}\left(I_{1} \times H^{1}(\mathbb{R}) \cap L_{\sigma}^{2}(\mathbb{R}), H^{1}(\mathbb{R}) \cap L_{\sigma}^{2}(\mathbb{R})\right)$.

A seguir, vamos demonstrar o Teorema 4.2.1.

Demonstração: No Lema 4.2.2, provamos que

$$
\mathscr{K}(E, r, h) \in C^{1}\left(I_{1} \times I_{2} \times\left(H^{1}(\mathbb{R}) \cap L_{\sigma}^{2}(\mathbb{R})\right), H^{1}(\mathbb{R}) \cap L_{\sigma}^{2}(\mathbb{R})\right) .
$$

Além disso, temos que 
- $\mathscr{H}\left(E_{0}, 0,0\right)=0-\lambda\left(H_{q}-E_{0}\right)^{-1} f_{c}(0,0)=0$

- $D_{h} \mathscr{K}\left(E_{0}, 0,0\right)=\left.D_{h} \mathscr{K}(E, r, h)\right|_{(E, r, h)=\left(E_{0}, 0,0\right)}=I$.

Logo, do Teorema da Função Implícita, temos

(i) existem abertos $U=B(0, \epsilon)=\left\{h \in L_{\sigma}^{2} \cap H^{1}:\|h\|_{L_{\sigma}^{2} \cap H^{1}}<\epsilon\right\}, V=\left(E_{0}-\mu, E_{0}+\mu\right) \times(-\mu, \mu) \subset$ $I_{1} \times I_{2}$ e uma única aplicação $\tilde{h}: V \rightarrow U$ tais que para todo $(E, r) \in V$

$$
\mathscr{K}(E, r, \tilde{h}(E, r))=0 .
$$

(ii) a aplicação $\tilde{h}$ é de classe $C^{1}$ em $V, \tilde{h}(E, 0)=0$, para todo $E \in I_{1} \mathrm{e}$

$$
\begin{aligned}
& D \tilde{h}(E, 0)=\left[\partial_{E} \tilde{h}(E, 0) \quad \partial_{r} \tilde{h}\left(E_{0}, 0\right)\right]=-\left(D_{h} \mathscr{K}(E, 0,0)\right)^{-1} D_{(E, r)} \mathscr{K}(E, 0,0)=-D_{(E, r)} \mathscr{K}(E, 0,0), \\
& \text { onde }-D_{(E, r)} \mathscr{K}(E, 0,0)=-\left[\partial_{E} \mathscr{K}(E, 0,0) \quad \partial_{r} \mathscr{K}(E, 0,0)\right] \text {. }
\end{aligned}
$$

Agora, substituindo $\tilde{h}(E, r)$ na segunda equação de (4.5), obtemos

$$
E_{0}-E=\lambda r^{-1} f_{p}(r, \tilde{h}(E, r))=\lambda \frac{\left\langle\left|r \psi_{0}+\tilde{h}(E, r)\right|^{2}\left(r \psi_{0}+\tilde{h}(E, r)\right), \psi_{0}\right\rangle}{r} .
$$

Logo, podemos considerar

$$
\begin{aligned}
\mathscr{J}: V \subseteq I_{1} \times I_{2} & \rightarrow I_{\delta}=(-\delta, \delta) \\
(E, r) & \mapsto \mathscr{J}(E, r)=E_{0}-E-\lambda \frac{\left\langle\left|r \psi_{0}+\tilde{h}(E, r)\right|^{p}\left(r \psi_{0}+\tilde{h}(E, r)\right), \psi_{0}\right\rangle}{r} .
\end{aligned}
$$

Veja que claramente, exceto para $r \neq 0, \mathscr{J}$ é uma função de classe $C^{1}\left(V, I_{\delta}\right)$. Veremos que $\mathscr{J}$ é diferenciável em $r=0$. Provamos anteriormente que $\partial_{r} \tilde{h}(E, 0)=-\partial_{r} \mathscr{K}(E, 0,0)=0$, assim como $\tilde{h}(E, 0)=0$, para todo $E \in I_{1}, \tilde{h}(E, r)=o(r)$, quando $r \rightarrow 0$ (Para a definição de $o$, veja [16, pag. 704]). Note que

$$
|| r \psi_{0}+\left.\left.\tilde{h}(E, r)\right|^{p}\left(\psi_{0}+\frac{\tilde{h}(E, r)}{r}\right)|=| r\right|^{p}\left|\psi_{0}+\frac{\tilde{h}(E, r)}{r}\right|^{p+1},
$$

mas, para $r$ suficientemente próximo de $0,\left|\psi_{0}+\frac{\tilde{h}(E, r)}{r}\right|^{p+1} \leq C$, então

$$
\mathscr{J}(E, 0)=\lim _{r \rightarrow 0} \mathscr{J}(E, r)=E_{0}-E .
$$

Além disso, temos

$$
\begin{gathered}
\partial_{E} \mathscr{J}(E, r)=-1-\lambda \frac{\left\langle(p+1)\left|r \psi_{0}+\tilde{h}(E, r)\right|^{p}\left(\partial_{E} \tilde{h}(E, r)\right), \psi_{0}\right\rangle}{r}, \\
\partial_{r} \mathscr{J}(E, r)=-\lambda\left\langle\frac{(p+1)\left|r \psi_{0}+\tilde{h}(E, r)\right|^{p}\left(\partial_{r} \tilde{h}(E, r)\right)-\left|r \psi_{0}+\tilde{h}(E, r)\right|^{p}\left(r \psi_{0}+\tilde{h}(E, r)\right)}{r^{2}}, \psi_{0}\right\rangle,
\end{gathered}
$$


assim

$$
\begin{gathered}
\partial_{E} \mathscr{J}(E, 0)=\lim _{r \rightarrow 0} \partial_{E} \mathscr{J}(E, r)=-1, \\
\partial_{r} \mathscr{J}(E, 0)=\lim _{r \rightarrow 0} \partial_{r} \mathscr{J}(E, r)=0 .
\end{gathered}
$$

Logo, $\mathscr{J}$ é uma função de classe $C^{1}\left(V, I_{\delta}\right)$. Observe que

- $\mathscr{J}\left(E_{0}, 0\right)=0$

- $\partial_{E} \mathscr{J}\left(E_{0}, 0\right)=\left.\partial_{E} \mathscr{J}(E, 0)\right|_{(E, r)=\left(E_{0}, 0\right)}=-1$.

Desta forma do Teorema da Função Implícita temos

(i) existem abertos $W=\left(E_{0}-\epsilon_{0}, E_{0}+\epsilon_{0}\right) \subseteq I_{1}, U_{1}=\left(-\epsilon_{1}, \epsilon_{1}\right) \subseteq I_{2}$ e uma única função $E: U_{1} \rightarrow W$ tais que para todo $r \in U_{1}$

$$
\mathscr{J}(E(r), r)=0 .
$$

(ii) a aplicação $E$ é de classe $C^{1}$ em $U_{1}$ e

- $E(0)=E_{0}$

- $E^{\prime}(0)=-\left(\partial_{E} \mathscr{J}\left(E_{0}, 0\right)\right)^{-1} \partial_{r} \mathscr{J}\left(E_{0}, 0\right)=\partial_{r} \mathscr{J}\left(E_{0}, 0\right)=0$.

Finalmente, de (4.15), obtemos nossa desejada relação

$$
E_{0}-E(r)=\lambda \frac{\left\langle\left|r \psi_{0}+\tilde{h}(E(r), r)\right|^{p}\left(r \psi_{0}+\tilde{h}(E(r), r)\right), \psi_{0}\right\rangle}{r}, \forall r \in U_{1} .
$$

Sendo assim, definimos para $u_{p} \in B(0, \delta) \subseteq \mathbb{C}, u_{p} \neq 0, \delta$ pequeno,

$$
h\left(u_{p}\right)=\frac{u_{p}}{\left|u_{p}\right|} \tilde{h}\left(E\left(\left|u_{p}\right|\right),\left|u_{p}\right|\right), \text { com }\left|u_{p}\right|<\delta,
$$

então $h\left(u_{p}\right)$ e $E=E\left(\left|u_{p}\right|\right)$ satisfazem o sistema (4.5). Note que provamos que $E(r)$ é de classe $C^{1}$ assim como $\tilde{h}$; desta forma, segue-se que $h$ também é de classe $C^{1}$. 


\title{
A NLS- $\delta$ em espaço de Sobolev com
}

\author{
peso
}

Neste capítulo, temos como objetivo estudar a parte dispersiva do grupo associado ao operador $H_{q}$ e a solução sobre a parte espectral de (3.1), ambos sobre espaços de Sobolev com peso. Todo esse estudo será necessário para provar o Teorema 6.2.1 sobre a aproximação à variedade invariante centro do próximo capítulo.

Inicialmente faremos algumas considerações e, em seguida, apresentaremos algumas estimativas dispersivas do grupo $\left\{e^{i t H_{q}}\right\}_{t \in \mathbb{R}}$ em espaço de Sobolev com peso, a saber, $H_{m}^{1}(\mathbb{R})$ e $H_{m}^{2}(\Omega)$, $m \geq 1$ inteiro. Tais estimativas serão utilizadas na última seção, onde veremos propriedades para as soluções no espectro contínuo do problema de valor inicial (3.1).

Neste capítulo chamamos uma atenção especial às notações $p$ e p. O índice p (romano) será utilizado para denotar o índice do espaço de Lebesgue e o $p$ (itálico) para denotar a potência da não linearidade do problema (3.1).

\subsection{O espaço $L^{\mathrm{p}}\left(I, L^{r}\right)$}

Para p, $r \in[1, \infty)$, definimos $L^{\mathrm{p}}\left(I, L^{r}\right)$ o espaço de todas as funções mensuráveis $f: I \rightarrow L^{r}$ tal que $\int_{I}\|f(t)\|_{r}^{\mathrm{p}} d t<\infty$, onde $I \subseteq \mathbb{R}$. Com a norma

$$
\|f\|_{L_{t}^{\mathrm{p}}(I) L_{x}^{r}}=\left(\int_{I}\|f(t)\|_{r}^{\mathrm{p}} d t\right)^{\frac{1}{\mathrm{p}}}
$$

o espaço $L^{\mathrm{p}}\left(I, L^{r}\right)$ é um espaço de Banach. Definimos $L^{\infty}\left(I, L^{r}\right)$ o espaço de todas as funções mensuráveis $f: I \rightarrow X$ tal que $t \mapsto\|f(t)\|_{r}$ é essencialmente limitada em $I \subseteq \mathbb{R}$. O espaço $L^{\infty}\left(I, L^{r}\right)$ com a norma

$$
\|f\|_{L_{t}^{\infty}(I) L_{x}^{r}}=\operatorname{esssup}_{t \in I}\|f(t)\|_{r} .
$$

é um espaço de Banach. Quando $I=[0, T]$, diremos que $f \in L^{\mathrm{p}}\left(0, T ; L^{r}\right)$ e sua norma será denotada por $\|f\|_{L_{T}^{\mathrm{p}} L_{x}^{r}}$. Nessa definição podemos trocar $L^{r}$ por um espaço de Banach qualquer. 


\subsection{Espaços de Sobolev com peso}

Sejam $k$ e $m$ números inteiros não negativos. Denotamos por $W_{m}^{k, \mathrm{p}}=W_{m}^{k, \mathrm{p}}(\mathbb{R})$ o espaço de Banach complexo com a norma

$$
\|\phi\|_{W_{m}^{k, \mathrm{p}}}=\left(\sum_{\alpha=0}^{k}\left\|\partial^{\alpha} \phi\right\|_{\mathrm{p}}^{\mathrm{p}}+\sum_{\alpha=0}^{m}\left\|x^{\alpha} \phi\right\|_{\mathrm{p}}^{\mathrm{p}}\right)^{\frac{1}{\mathrm{p}}} .
$$

Quando $\mathrm{p}=2$, denotamos $H_{m}^{k}=H_{m}^{k}(\mathbb{R})=W_{m}^{k, 2}(\mathbb{R})$.

Consideremos $U_{q}(t)=e^{-i t H_{q}}-e^{-i t \frac{q^{2}}{2}} P_{p}$.

Proposição 5.1 (Estimativa de Strichartz). Se $2 \leq \mathrm{p}^{\prime}, r \leq \infty e \frac{2}{r}=\frac{1}{2}-\frac{1}{\mathrm{p}^{\prime}}$, então

$$
\left\|U_{0}(\cdot) \phi\right\|_{L_{t}^{r}(\mathbb{R}) L_{x}^{\mathrm{p}^{\prime}}} \leq c\|\phi\|_{2}
$$

para toda $\phi \in L^{2}$.

Demonstração: Veja [11, Teorema 2.3.3].

Proposição 5.2. Se $2 \leq \mathrm{p}^{\prime}, r \leq \infty e \frac{2}{r}=\frac{1}{2}-\frac{1}{\mathrm{p}^{\prime}}$, então

$$
\left\|U_{q}(\cdot) \phi\right\|_{L_{t}^{r}(\mathbb{R}) L_{x}^{\mathrm{p}^{\prime}}} \leq c\|\phi\|_{2}
$$

para toda $\phi \in L^{2}$.

Demonstração: Seja $\phi \in L^{2}(\mathbb{R})$, do Lema 3.2.1,

$$
\begin{aligned}
\left\|U_{q}(\cdot) \phi\right\|_{L_{t}^{r}(\mathbb{R}) L_{x}^{\mathrm{p}^{\prime}}} \leq & \left\|U_{0}(\cdot)\left(\phi * \tau_{q}\right) \chi_{[0, \infty)}\right\|_{L_{t}^{r}(\mathbb{R}) L_{x}^{\mathrm{p}^{\prime}}}+\left\|U_{0}(\cdot) \phi \chi_{(-\infty, 0]}\right\|_{L_{t}^{r}(\mathbb{R}) L_{x}^{\mathrm{p}^{\prime}}} \\
& +\left\|U_{0}(\cdot)\left(\phi * \varrho_{q}\right)(-\bullet) \chi_{(-\infty, 0]}\right\|_{L_{t}^{r}(\mathbb{R}) L_{x}^{\mathrm{p}^{\prime}}},
\end{aligned}
$$

onde $\tau_{q}$ e $\varrho_{q}$ são dadas na equação (3.32). Note que

$$
\phi * \tau_{q}=\phi *\left(\delta_{0}(x)+\varrho_{q}\right)=\phi * \delta_{0}(x)+\phi * \varrho_{q}=\phi+\phi * \varrho_{q},
$$

assim, da Proposição 5.1,

$$
\left\|U_{q}(\cdot) \phi\right\|_{L_{t}^{r}(\mathbb{R}) L_{x}^{\mathrm{p}^{\prime}}} \leq c\left\|\phi+\phi * \varrho_{q}\right\|_{2}+c\|\phi\|_{2}+c\left\|\left(\phi * \varrho_{q}\right)(-\bullet)\right\|_{2},
$$

pela desigualdade de Young,

$$
\begin{aligned}
\left\|U_{q}(\cdot) \phi\right\|_{L_{t}^{r}(\mathbb{R}) L_{x}^{\mathrm{p}^{\prime}}} & \leq c\|\phi\|_{2}+c\|\phi\|_{2}\left\|\varrho_{q}\right\|_{1}+c\|\phi\|_{2}+c\|\phi\|_{2}\left\|\varrho_{q}(-\bullet)\right\|_{1}, \\
& \leq C\|\phi\|_{2} .
\end{aligned}
$$


Observação 5.2.1. Na demonstração anterior, vimos que

$$
\phi * \tau_{q}=\phi+\phi * \varrho_{q}
$$

Por outro lado, pelo Lema 3.2.1, sendo $\phi \in L^{1}(\mathbb{R})$, com supp $\phi \subset(-\infty, 0]$, então

$$
\begin{aligned}
e^{-i t H_{q}} \phi(x)= & e^{i t \frac{q^{2}}{2}} P_{p} \phi(x)+e^{-i t H_{0}}\left(\phi * \tau_{q}\right)(x) \chi_{[0, \infty)}(x) \\
& +e^{-i t H_{0}} \phi(x) \chi_{(-\infty, 0]}(x)+e^{-i t H_{0}}\left(\phi * \varrho_{q}\right)(-x) \chi_{(-\infty, 0]}(x) .
\end{aligned}
$$

$\log$,

$$
\begin{aligned}
e^{-i t H_{0}}\left(\phi * \tau_{q}\right)(x) \chi_{[0, \infty)}(x) & =e^{-i t H_{0}}\left(\phi+\phi * \varrho_{q}\right)(x) \chi_{[0, \infty)}(x) \\
& =e^{-i t H_{0}} \phi(x) \chi_{[0, \infty)}(x)+e^{-i t H_{0}}\left(\phi * \varrho_{q}\right)(x) \chi_{[0, \infty)}(x) .
\end{aligned}
$$

Mas

$$
e^{-i t H_{0}} \phi(x) \chi_{(-\infty, 0]}(x)+e^{-i t H_{0}} \phi(x) \chi_{[0, \infty)}(x)=e^{-i t H_{0}} \phi(x)
$$

$e$

$$
\begin{aligned}
& e^{-i t H_{0}}\left(\phi * \varrho_{q}\right)(-x) \chi_{(-\infty, 0]}(x)+e^{-i t H_{0}}\left(\phi * \tau_{q}\right)(x) \chi_{[0, \infty)}(x) \\
= & e^{-i t H_{0}}\left(\phi * \varrho_{q}\right)(|x|) \chi_{(-\infty, 0]}(x)+e^{-i t H_{0}}\left(\phi * \tau_{q}\right)(|x|) \chi_{[0, \infty)}(x) \\
= & e^{-i t H_{0}}\left(\phi * \varrho_{q}\right)(|x|) .
\end{aligned}
$$

Portanto,

$$
e^{-i t H_{q}} \phi(x)=e^{i t \frac{q^{2}}{2}} P_{p} \phi(x)+e^{-i t H_{0}} \phi(x)+e^{-i t H_{0}}\left(\phi * \varrho_{q}\right)(|x|),
$$

ou seja, o Lema 3.2.1 nos fornece como podemos escrever $U_{q}$ em termos de $U_{0}$ e das estimativas para $U_{0}$ são obtidas de [24].

Lema 5.2.1. Sejam $2 \leq \mathrm{p}^{\prime}, r \leq \infty \operatorname{com} \frac{2}{r}=\frac{1}{2}-\frac{1}{\mathrm{p}^{\prime}}$ e $T>0$. Então, para

$$
I(t, f) \equiv \int_{0}^{t} U_{q}(t-s) f(s) d s,
$$

temos

$$
\|I(\cdot, f)\|_{L_{T}^{r} L_{x}^{\mathrm{p}^{\prime}}} \leq c\|f\|_{L_{T}^{r^{\prime}} L_{x}^{\mathrm{p}}}
$$

para toda $f \in L^{r^{\prime}}\left(0, T ; L^{\mathrm{p}}\right)$, onde $\frac{1}{r}+\frac{1}{r^{\prime}}=1 e \frac{1}{\mathrm{p}}+\frac{1}{\mathrm{p}^{\prime}}=1, e$

$$
\|I(t, f)\|_{2} \leq c\|f\|_{L_{T}^{r^{\prime}} L_{x}^{\mathrm{p}}},
$$


para toda $f \in L^{r^{\prime}}\left(0, T ; L^{\mathrm{p}}\right)$ e todo $t \in[0, T]$. Além disso, para cada $f \in L^{r^{\prime}}\left(0, T ; L^{\mathrm{p}}\right), I(\cdot, f) \in$ $C\left([0, T] ; L^{2}\right)$.

Demonstração: Se $\mathrm{p}^{\prime}=2$, basta ver que $\left\|U_{q}(t) \phi\right\|_{2} \leq\|\phi\|_{2}$. Logo, consideremos $2<\mathrm{p}^{\prime} \leq \infty$, assim

$$
\begin{aligned}
\|I(\cdot, f)\|_{L_{T}^{r} L_{x}^{\mathrm{p}^{\prime}}} & \leq\left(\int_{0}^{T}\left[\int_{0}^{t}\left\|U_{q}(t-s) f(s)\right\|_{\mathrm{p}^{\prime}} d s\right]^{r} d t\right)^{\frac{1}{r}} \\
& \leq\left(\int_{0}^{T}\left[\int_{0}^{t} C|t-s|^{-\left(\frac{1}{2}-\frac{1}{\mathrm{p}^{\prime}}\right)}\|f(s)\|_{\mathrm{p}} d s\right]^{r} d t\right)^{\frac{1}{r}}
\end{aligned}
$$

mas, pela desigualdade de Hardy-Littlewood-Sobolev,

$$
\left(\int_{0}^{T}\left[\int_{0}^{t} C|t-s|^{-\left(\frac{1}{2}-\frac{1}{\mathrm{p}^{\prime}}\right)}\|f(s)\|_{\mathrm{p}} d s\right]^{r} d t\right)^{\frac{1}{r}} \leq c\left(\int_{0}^{T}\|f(s)\|_{\mathrm{p}}^{r^{\prime}} d s\right)^{\frac{1}{r^{\prime}}}=c\|f\|_{L_{T}^{r^{\prime}} L_{x}^{\mathrm{p}}},
$$

pois

- $\frac{1}{2}-\frac{1}{\mathrm{p}^{\prime}}=1-\left(\frac{1}{2}+\frac{1}{\mathrm{p}^{\prime}}\right)$ e $\frac{1}{2}+\frac{1}{\mathrm{p}^{\prime}}<1$

- $\frac{2}{r}=\frac{1}{2}-\frac{1}{\mathrm{p}^{\prime}} \Rightarrow \frac{1}{r}=-1+\frac{1}{r^{\prime}}+\frac{1}{2}-\frac{1}{\mathrm{p}^{\prime}}=\frac{1}{r^{\prime}}-\left(\frac{1}{2}+\frac{1}{\mathrm{p}^{\prime}}\right)$.

Logo,

$$
\|I(\cdot, f)\|_{L_{T}^{r} L_{x}^{\mathrm{p}^{\prime}}} \leq c\|f\|_{L_{T}^{r^{\prime}} L_{x}^{\mathrm{p}}}
$$

Para mostrarmos (5.5), por densidade e dualidade precisamos somente mostrar que, para toda $\phi \in \mathscr{S}(\mathbb{R})$ e para todo $t \in[0, T]$

$$
\langle\phi, I(t, f)\rangle \leq c\|\phi\|_{2}\|f\|_{L_{T}^{r^{\prime}} L_{x}^{\mathrm{p}}}
$$

$\langle\cdot, \cdot\rangle$ representa a dualidade com $\langle\psi, \varphi\rangle=\int \psi(x) \varphi(x) d x$. A partir da demonstração acima, temos $I(t, f) \in L^{\mathrm{p}^{\prime}}$, para quase todo $t \in[0, T]$. Então,

$$
\begin{aligned}
|\langle\phi, I(t, f)\rangle| & =\left|\left\langle\phi, \int_{0}^{t} U_{q}(t-s) f(s) d s\right\rangle\right|=\left|\int_{0}^{t}\left\langle U_{q}(t-s) \phi, f(s)\right\rangle d s\right| \\
& \leq \int_{0}^{t}\left|\left\langle U_{q}(t-s) \phi, f(s)\right\rangle\right| d s \leq \int_{0}^{t}\left\|U_{q}(t-s) \phi\right\|_{\mathrm{p}^{\prime}}\|f(s)\|_{\mathrm{p}} d s \\
& \leq\left(\int_{0}^{t}\left\|U_{q}(t-s) \phi\right\|_{\mathrm{p}^{\prime}}^{r} d s\right)^{\frac{1}{r}}\left(\int_{0}^{t}\|f(s)\|_{p}^{r^{\prime}} d s\right)^{\frac{1}{r^{\prime}}} \\
& \leq\left\|U_{q}(\cdot) \phi\right\|_{L_{t}^{r}(\mathbb{R}) L_{x}^{\mathrm{p}^{\prime}}}\|f\|_{L_{T}^{r^{\prime}} L_{x}^{\mathrm{p}}}
\end{aligned}
$$

pela Proposição 5.2,

$$
|\langle\phi, I(t, f)\rangle| \leq c\|\phi\|_{2}\|f\|_{L_{T}^{r^{\prime}} L_{x}^{\mathrm{p}}}
$$


obtendo (5.5). Por fim, resta-nos provar que

$$
I(\cdot, f) \in C\left([0, T] ; L^{2}\right)
$$

Sejam $f_{j} \in C_{0}([0, T] \times \mathbb{R})($ espaço das funções contínuas com suporte compacto), com $j=1,2, \ldots$, tais que

$$
\left\|f_{j}-f\right\|_{L_{T}^{r^{\prime}} L_{x}^{\mathrm{p}}} \rightarrow 0, \text { quando } j \rightarrow \infty .
$$

Então, $I\left(\cdot, f_{j}\right) \in C\left([0, T] ; L^{2}\right)$, pois $U_{q}(t)$ é unitário sobre $L^{2}$. Mais ainda, da equação $(5.5)$

$$
\left\|I\left(\cdot, f_{j}\right)-I(\cdot, f)\right\|_{L_{T}^{\infty} L_{x}^{2}} \leq c\left\|f_{j}-f\right\|_{L_{T}^{r^{\prime} L_{x}^{\mathrm{p}}}} \rightarrow 0, \text { quando } j \rightarrow \infty
$$

Portanto, $I(\cdot, f) \in C\left([0, T] ; L^{2}\right)$.

Para todo inteiro não negativo $m$ e para todo $\mathrm{p}$, com $1<\mathrm{p}<\infty$,

$$
\sum_{\alpha+\beta \leq m}\left\|x^{\alpha} \partial^{\beta} \phi\right\|_{\mathrm{p}} \leq c\|\phi\|_{W_{m}^{m, \mathrm{p}}}
$$

para toda $\phi \in W_{m}^{m, \mathrm{p}}$ (veja [44, Teorema 4]). Em particular,

$$
\left\|(x-i t \partial)^{\alpha} \phi\right\|_{\mathrm{p}} \leq C(1+|t|)^{m}\|\phi\|_{W_{m}^{m, \mathrm{p}}},
$$

sempre que $\alpha \leq m, t \in \mathbb{R}$ e $\phi \in W_{m}^{m, \mathrm{p}}$.

Proposição 5.3. Se $\phi \in H_{1}^{1}(\mathbb{R})$, então

$$
\phi * \tau_{q} \in H_{1}^{1}(\mathbb{R}) \text { e } \phi * \varrho_{q} \in H_{1}^{1}(\mathbb{R}),
$$

onde $\tau_{q}$ e $\varrho_{q}$ são dadas no Lema 3.2.1.

Demonstração: Seja $\phi \in H_{1}^{1}(\mathbb{R})$. Já sabemos que

$$
\phi * \tau_{q}=\phi+\phi * \varrho_{q},
$$

então

$$
\begin{aligned}
\left\|\phi * \tau_{q}\right\|_{H_{1}^{1}}^{2} & =\left\|\phi+\phi * \varrho_{q}\right\|_{H_{1}^{1}}^{2} \\
& \leq 2\left(\|\phi\|_{H_{1}^{1}}^{2}+\left\|\phi * \varrho_{q}\right\|_{H_{1}^{1}}^{2}\right) \\
& =2\left(\|\phi\|_{H_{1}^{1}}^{2}+2\left\|\phi * \varrho_{q}\right\|_{2}^{2}+\left\|x\left(\phi * \varrho_{q}\right)\right\|_{2}^{2}+\left\|\frac{d}{d x}\left(\phi * \varrho_{q}\right)\right\|_{2}^{2}\right) \\
& =2\left(\|\phi\|_{H_{1}^{1}}^{2}+2\left\|\phi * \varrho_{q}\right\|_{2}^{2}+\left\|x\left(\phi * \varrho_{q}\right)\right\|_{2}^{2}+\left\|\left(\frac{d \phi}{d x} * \varrho_{q}\right)\right\|_{2}^{2}\right)
\end{aligned}
$$


mas, para todo $\alpha$ inteiro não negativo,

$$
\begin{aligned}
x^{\alpha}\left(\phi * \varrho_{q}\right)(x) & =\int_{-\infty}^{\infty} x^{\alpha} \phi(x-y) \varrho_{q}(y) d y=\int_{-\infty}^{\infty}((x-y)+y)^{\alpha} \phi(x-y) \varrho_{q}(y) d y \\
& \leq C(\alpha) \int_{-\infty}^{\infty}(x-y)^{\alpha} \phi(x-y) \varrho_{q}(y) d y+C(\alpha) \int_{-\infty}^{\infty} \phi(x-y) y^{\alpha} \varrho_{q}(y) d y \\
& =C(\alpha)\left[\left(\left(x^{\alpha} \phi\right) * \varrho_{q}\right)(x)+\left(\phi * x^{\alpha} \varrho_{q}\right)(x)\right],
\end{aligned}
$$

então, usando a desigualdade de Young, com $\alpha=0$ e $\alpha=1$,

$$
\begin{aligned}
\left\|x^{\alpha}\left(\phi * \varrho_{q}\right)\right\|_{2}^{2} & \leq C(\alpha)\left[\left\|\left(x^{\alpha} \phi\right) * \varrho_{q}\right\|_{2}^{2}+\left\|\phi * x^{\alpha} \varrho_{q}\right\|_{2}^{2}\right] \\
& \leq C(\alpha)\left[\left\|x^{\alpha} \phi\right\|_{2}^{2}\left\|\varrho_{q}\right\|_{1}^{2}+\|\phi\|_{2}^{2}\left\|x^{\alpha} \varrho_{q}\right\|_{1}^{2}\right] \\
& \leq C(\alpha)\|\phi\|_{H_{1}^{1}}^{2}
\end{aligned}
$$

e

$$
\left\|\left(\frac{d \phi}{d x} * \varrho_{q}\right)\right\|_{2}^{2} \leq\left\|\frac{d \phi}{d x}\right\|_{2}^{2}\left\|\varrho_{q}\right\|_{1}^{2} \leq C\|\phi\|_{H_{1}^{1}}^{2}
$$

$\log$,

$$
\left\|\phi * \tau_{q}\right\|_{H_{1}^{1}}^{2} \leq C(\alpha)\|\phi\|_{H_{1}^{1}}^{2}
$$

ou seja,

$$
\phi * \tau_{q} \in H_{1}^{1}(\mathbb{R}) .
$$

Da demonstração dada, obtemos imediatamente $\phi * \varrho_{q} \in H_{1}^{1}(\mathbb{R})$.

Observação 5.2.2. Na Proposição anterior, mostramos que para $\mathrm{p}=2$ e $k=1$, se $\phi \in W_{k}^{1, \mathrm{p}}(\mathbb{R})$, então $\phi * \tau_{q} \in W_{k}^{1, \mathrm{p}}(\mathbb{R})$ e $\phi * \varrho_{q} \in W_{k}^{1, \mathrm{p}}(\mathbb{R})$, mas, note que se consideramos $\mathrm{p} \geq 1$ e $k$ um inteiro não negativo fixo, se $\phi \in W_{k}^{1, \mathrm{p}}(\mathbb{R})$, então $\phi * \tau_{q} \in W_{k}^{1, \mathrm{p}}(\mathbb{R})$ e $\phi * \varrho_{q} \in W_{k}^{1, \mathrm{p}}(\mathbb{R})$.

Lema 5.2.2. Seja $2 \leq \mathrm{p}^{\prime} \leq \infty$. Para todo $t \in \mathbb{R}, U_{q}(t): W_{1}^{1, \mathrm{p}} \rightarrow W_{1}^{1, \mathrm{p}^{\prime}}$ é um operador limitado, com $\frac{1}{\mathrm{p}}+\frac{1}{\mathrm{p}^{\prime}}=1$. Além disso, para qualquer $t, s \in \mathbb{R}$ com $t \neq s$ e para toda $\phi \in W_{1}^{1, \mathrm{p}}$, temos

$$
(x+i t \partial) U_{q}(t-s) \phi(x)=U_{0}(t-s)(x+i s \partial) \phi(x)+\operatorname{sign}(x) U_{0}(t-s)(x+i s \partial)\left(\phi * \varrho_{q}\right)(|x|) .
$$

Para $\mathrm{p}^{\prime}=2, U_{q}(t)$ é limitado para todo $t \in \mathbb{R}$ e a equação (5.8) vale para todo $t, s \in \mathbb{R}$.

Demonstração: Se $q=0,[24]$ mostra que

$$
\begin{aligned}
(x+i t \partial) U_{0}(t-s) \psi(x) & =U_{0}(t-s)(x+i s \partial) \psi(x) \\
(x+i t \partial) U_{0}(t-s) \psi(-x) & =-U_{0}(t-s)(x+i s \partial) \psi(-x),
\end{aligned}
$$

para toda $\psi \in W_{1}^{1, \mathrm{p}}$ e para todo $t \in \mathbb{R}, U_{0}(t): W_{1}^{1, \mathrm{p}} \rightarrow W_{1}^{1, \mathrm{p}^{\prime}}$ é um operador limitado, com 
$\frac{1}{\mathrm{p}}+\frac{1}{\mathrm{p}^{\prime}}=1$. Desta maneira, de (5.3), para toda $\phi \in W_{1}^{1, \mathrm{p}}$,

$$
\begin{aligned}
\left\|U_{q}(t) \phi\right\|_{W_{1}^{1, \mathrm{p}^{\prime}}} & \leq\left\|U_{0}(t) \phi\right\|_{W_{1}^{1, \mathrm{p}^{\prime}}}+\left\|U_{0}(t)\left(\phi * \varrho_{q}\right)(|\bullet|)\right\|_{W_{1}^{1, \mathrm{p}^{\prime}}} \\
& \leq c\left[\|\phi\|_{W_{1}^{1, \mathrm{p}}}+\left\|\left(\phi * \varrho_{q}\right)(|\bullet|)\right\|_{W_{1}^{1, \mathrm{p}}}\right] \\
& \leq C\|\phi\|_{W_{1}^{1, \mathrm{p}}}
\end{aligned}
$$

pois $\frac{d}{d x}\left[\left(\phi * \varrho_{q}\right)(|x|)\right]=\operatorname{sign}(x)\left(\frac{d}{d x} \phi * \varrho_{q}\right)(|x|)$.

Para $q<0$, pelo Lema 3.2.1, se consideramos $x<0$, obtemos

$$
(x+i t \partial) U_{q}(t-s) \phi(x)=(x+i t \partial) U_{0}(t-s) \phi(x)+(x+i t \partial) U_{0}(t-s)\left(\phi * \varrho_{q}\right)(-x),
$$

então pela Proposição 5.3 e (5.9), temos

$$
(x+i t \partial) U_{q}(t-s) \phi(x)=U_{0}(t-s)(x+i s \partial) \phi(x)-U_{0}(t-s)(x+2 i s \partial)\left(\phi * \varrho_{q}\right)(-x)
$$

por outro lado, para $x>0$, temos, por um procedimento similar ao feito em (5.10),

$$
\begin{aligned}
(x+i t \partial) U_{q}(t-s) \phi & =(x+i t \partial) U_{0}(t-s)\left(\phi * \tau_{q}\right)(x) \\
& =(x+i t \partial) U_{0}(t-s)\left(\phi+\phi * \varrho_{q}\right)(x) \\
& =(x+i t \partial) U_{0}(t-s) \phi(x)+(x+i t \partial) U_{0}(t-s)\left(\phi * \varrho_{q}\right)(x) \\
& =U_{0}(t-s)(x+i s \partial) \phi(x)+U_{0}(t-s)(x+i s \partial)\left(\phi * \varrho_{q}\right)(x),
\end{aligned}
$$

portanto,

$$
(x+i t \partial) U_{q}(t-s) \phi=U_{0}(t-s)(x+i s \partial) \phi+\operatorname{sign}(x) U_{0}(t-s)(x+i s \partial)\left(\phi * \varrho_{q}\right)(|x|) .
$$

Vamos estender agora o Lema anterior para o espaço $W_{m}^{1, \mathrm{p}^{\prime}}(\mathbb{R})$.

Lema 5.2.3. Seja $2 \leq \mathrm{p}^{\prime} \leq \infty$. Para todo $t \in \mathbb{R}, U_{q}(t): W_{m}^{1, \mathrm{p}} \rightarrow W_{m}^{1, \mathrm{p}^{\prime}}$ é um operador limitado, com $\frac{1}{\mathrm{p}}+\frac{1}{\mathrm{p}^{\prime}}=1$. Além disso, para qualquer $t, s \in \mathbb{R}$ com $t \neq s$ e para toda $\phi \in W_{1}^{1, \mathrm{p}}$, temos

$$
(x+i t \partial) U_{q}(t-s) \phi(x)=U_{0}(t-s)(x+i s \partial) \phi(x)+\operatorname{sign}(x) U_{0}(t-s)(x+i s \partial)\left(\phi * \varrho_{q}\right)(|x|) .
$$

Para $\mathrm{p}^{\prime}=2, U_{q}(t)$ é limitado para todo $t \in \mathbb{R}$ e a equação (5.11) vale para todo $t, s \in \mathbb{R}$.

Demonstração: Se $q=0,[24]$ mostra que

$$
\begin{aligned}
(x+i t \partial) U_{0}(t-s) \psi(x) & =U_{0}(t-s)(x+i s \partial) \psi(x) \\
(x+i t \partial) U_{0}(t-s) \psi(-x) & =-U_{0}(t-s)(x+i s \partial) \psi(-x),
\end{aligned}
$$


para toda $\psi \in W_{m}^{1, \mathrm{p}}$ e para todo $t \in \mathbb{R}, U_{0}(t): W_{m}^{1, \mathrm{p}} \rightarrow W_{m}^{1, \mathrm{p}^{\prime}}$ é um operador limitado, com $\frac{1}{\mathrm{p}}+\frac{1}{\mathrm{p}^{\prime}}=1$. Desta maneira, de (5.3) e da Observação 5.2.2, para toda $\phi \in W_{m}^{1, \mathrm{p}}$,

$$
\left\|U_{q}(t) \phi\right\|_{W_{m}^{1, \mathrm{p}^{\prime}}} \leq C\|\phi\|_{W_{m}^{1, \mathrm{p}}}
$$

A demonstração de (5.11) é análoga à feita no Lema 5.2.2.

Vejamos mais algumas propriedades de $U_{q}(t)$ no espaço $W_{1}^{1, \mathrm{p}^{\prime}}$.

Lema 5.2.4. Para qualquer $\mathrm{p}^{\prime}$ com $2 \leq \mathrm{p}^{\prime} \leq \infty$ e para qualquer $t \in \mathbb{R} \backslash\{0\}, U_{q}(t): W_{1}^{1, \mathrm{p}} \rightarrow W_{1}^{1, \mathrm{p}^{\prime}}$ é um um operador limitado com

$$
\left\|U_{q}(t) \phi\right\|_{W_{1}^{1, \mathrm{p}^{\prime}}} \leq c(1+|t|)|t|^{-\left(\frac{1}{\mathrm{p}}-\frac{1}{2}\right)}\|\phi\|_{W_{1}^{1, \mathrm{p}}}
$$

para toda $\phi \in W_{1}^{1, \mathrm{p}}$ e a aplicação $t \mapsto U_{q}(t)$ é fortemente contínua. Se $\mathrm{p}^{\prime}=2$, as afirmações vale para todo $t \in \mathbb{R}$. Mais ainda, para quaisquer $\mathrm{p}^{\prime}$ e $r$ satisfazendo $2 \leq \mathrm{p}^{\prime}<\infty, 2 \leq r \leq \infty e$ $\frac{2}{r}=\frac{1}{2}-\frac{1}{\mathrm{p}^{\prime}}=\frac{1}{\mathrm{p}}-\frac{1}{2}$, com

$$
I(t, u) \equiv \int_{0}^{t} U_{q}(t-s) u(s) d s,
$$

obtemos as seguintes desigualdades

$$
\begin{aligned}
& \left\|U_{q}(\cdot) \phi\right\|_{L_{T}^{r} W_{1}^{1, \mathrm{p}^{\prime}}} \leq c(1+T)\|\phi\|_{W_{1}^{1, \mathrm{p}}}, \quad \forall \phi \in W_{1}^{1, \mathrm{p}} \\
& \|I(\cdot, u)\|_{L_{T}^{r} W_{1}^{1, \mathrm{p}^{\prime}}} \leq c(1+T)\|u\|_{L_{T}^{r^{\prime}} W_{1}^{1, \mathrm{p}}}, \quad \forall u \in L^{r^{\prime}}\left(0, T ; W_{1}^{1, \mathrm{p}}\right) \\
& \|I(t, u)\|_{H_{1}^{1}} \leq c(1+T)\|u\|_{L_{T}^{r^{\prime}} W_{1}^{1, \mathrm{p}}}, \quad \forall u \in L^{r^{\prime}}\left(0, T ; W_{1}^{1, \mathrm{p}}\right),
\end{aligned}
$$

para todo $t \in[0, T] e \frac{1}{r}+\frac{1}{r^{\prime}}=1$. Mais ainda, para cada $u \in L^{r^{\prime}}\left(0, T ; W^{1, \mathrm{p}}\right)$, temos $I(\cdot, u) \in$ $C\left([0, T] ; H_{1}^{1}\right)$.

Demonstração: Usando as equações (5.21) e (5.7), temos

$$
\begin{aligned}
(x+i t \partial) U_{q}(t-s) \phi(x) & =U_{0}(t-s)(x+i s \partial) \phi(x)+\operatorname{sign}(x) U_{0}(t-s)(x+i s \partial)\left(\phi * \varrho_{q}\right)(|x|) \\
\|(x-i t \partial) \phi\|_{\mathrm{p}} & \leq C(1+|t|)\|\phi\|_{W_{1}^{1, \mathrm{p}}}
\end{aligned}
$$

para todo $t \in \mathbb{R}$ e para toda $\phi \in W_{1}^{1, \mathrm{p}}$. Se $t=0$, da primeira equação acima teremos

$$
x U_{q}(-s) \phi(x)=U_{0}(-s)(x+i s \partial) \phi(x)+\operatorname{sign}(x) U_{0}(-s)(x+i s \partial)\left(\phi * \varrho_{q}\right)(|x|),
$$

agora, chamando $s=-t$ na última relação, obtemos

$$
x U_{q}(t) \phi(x)=U_{0}(t)(x-i t \partial) \phi(x)+\operatorname{sign}(x) U_{0}(t)(x-i t \partial)\left(\phi * \varrho_{q}\right)(|x|) .
$$


Sejam $\phi \in W_{1}^{1, \mathrm{p}}, 2 \leq \mathrm{p}^{\prime} \leq \infty$ e $t \in \mathbb{R}$. Se $p^{\prime} \neq 2$, suponhamos que $t \neq 0$. Em $L^{\mathrm{p}^{\prime}}$, de (5.3),

$$
\begin{aligned}
\partial U_{q}(t) \phi(x) & =e^{-i t H_{0}} \partial \phi(x)+\partial e^{-i t H_{0}}\left(\phi * \varrho_{q}\right)(|x|) \\
& =U_{0}(t) \partial \phi(x)+\operatorname{sign}(x) U_{0}(t)\left(\frac{d}{d x} \phi * \varrho_{q}\right)(|x|) .
\end{aligned}
$$

Então,

$$
\begin{aligned}
\left\|U_{q}(t) \phi\right\|_{W_{1}^{1, \mathrm{p}^{\prime}}}^{\mathrm{p}^{\prime}} & =\sum_{\alpha=0}^{1}\left[\left\|\partial^{\alpha} U_{q}(t) \phi\right\|_{\mathrm{p}^{\prime}}^{\mathrm{p}^{\prime}}+\left\|x^{\alpha} U_{q}(t) \phi\right\|_{\mathrm{p}^{\prime}}^{\mathrm{p}^{\prime}}\right] \\
& =2\left\|U_{q}(t) \phi\right\|_{\mathrm{p}^{\prime}}^{\mathrm{p}^{\prime}}+\left\|\partial U_{q}(t) \phi\right\|_{\mathrm{p}^{\prime}}^{\mathrm{p}^{\prime}}+\left\|x U_{q}(t) \phi\right\|_{\mathrm{p}^{\prime}}^{\mathrm{p}^{\prime}}
\end{aligned}
$$

Mas,

$$
\begin{aligned}
\left\|U_{q}(t) \phi\right\|_{\mathrm{p}^{\prime}}^{\mathrm{p}^{\prime}} & \leq c|t|^{-\mathrm{p}^{\prime}\left(\frac{1}{\mathrm{p}}-\frac{1}{2}\right)}\|\phi\|_{\mathrm{p}}^{\mathrm{p}^{\prime}} \leq c|t|^{-\mathrm{p}^{\prime}\left(\frac{1}{\mathrm{p}}-\frac{1}{2}\right)}\|\phi\|_{W_{1}^{1, \mathrm{p}}}^{\mathrm{p}^{\prime}} \\
\left\|\partial U_{q}(t) \phi\right\|_{\mathrm{p}^{\prime}}^{\mathrm{p}^{\prime}} & =\left\|U_{0}(t) \partial \phi+\operatorname{sign}(x) U_{0}(t)\left(\partial \phi * \varrho_{q}\right)(|\bullet|)\right\|_{\mathrm{p}^{\prime}}^{\mathrm{p}^{\prime}} \\
& \leq c\left(\left\|U_{0}(t) \partial \phi\right\|_{\mathrm{p}^{\prime}}^{\mathrm{p}^{\prime}}+\left\|\operatorname{sign}(x) U_{0}(t)\left(\partial \phi * \varrho_{q}\right)(|\bullet|)\right\|_{\mathrm{p}^{\prime}}^{\mathrm{p}^{\prime}}\right) \\
& =c\left(\left\|U_{0}(t) \partial \phi\right\|_{\mathrm{p}^{\prime}}^{\mathrm{p}^{\prime}}+\left\|U_{0}(t)\left(\partial \phi * \varrho_{q}\right)\right\|_{\mathrm{p}^{\prime}}^{\mathrm{p}^{\prime}}\right) \\
& \leq c|t|^{-\mathrm{p}^{\prime}\left(\frac{1}{\mathrm{p}}-\frac{1}{2}\right)}\left(\|\partial \phi\|_{\mathrm{p}}^{\mathrm{p}^{\prime}}+\left\|\partial \phi * \varrho_{q}\right\|_{\mathrm{p}}^{\mathrm{p}^{\prime}}\right) \\
& \leq c|t|^{-\mathrm{p}^{\prime}\left(\frac{1}{\mathrm{p}}-\frac{1}{2}\right)}\left(\|\partial \phi\|_{\mathrm{p}}^{\mathrm{p}^{\prime}}+\|\partial \phi\|_{\mathrm{p}}^{\mathrm{p}^{\prime}}\right) \\
& \leq c|t|^{-\mathrm{p}^{\prime}\left(\frac{1}{\mathrm{p}}-\frac{1}{2}\right)}\|\phi\|_{W_{1}^{1, \mathrm{p}}}^{\mathrm{p}^{\prime}}
\end{aligned}
$$

$\mathrm{e}$

$$
\begin{aligned}
\left\|x U_{q}(t) \phi\right\|_{\mathrm{p}^{\prime}}^{\mathrm{p}^{\prime}} & =\left\|U_{0}(t)(x-i t \partial) \phi+\operatorname{sign}(x) U_{0}(t)(x-i t \partial)\left(\phi * \varrho_{q}\right)(|\bullet|)\right\|_{\mathrm{p}^{\prime}}^{\mathrm{p}^{\prime}} \\
& \leq c\left\|U_{0}(t)(x-i t \partial) \phi\right\|_{\mathrm{p}^{\prime}}^{\mathrm{p}^{\prime}}+\left\|U_{0}(t)(x-i t \partial)\left(\phi * \varrho_{q}\right)\right\|_{\mathrm{p}^{\prime}}^{\mathrm{p}^{\prime}} \\
& \leq|t|^{-\mathrm{p}^{\prime}\left(\frac{1}{\mathrm{p}}-\frac{1}{2}\right)}\|(x-i t \partial) \phi\|_{\mathrm{p}}^{\mathrm{p}^{\prime}}+c|t|^{-\mathrm{p}^{\prime}\left(\frac{1}{\mathrm{p}}-\frac{1}{2}\right)}\left\|(x-i t \partial)\left(\phi * \varrho_{q}\right)\right\|_{\mathrm{p}}^{\mathrm{p}^{\prime}} \\
& \leq c(1+|t|)^{\mathrm{p}^{\prime}}|t|^{-\mathrm{p}^{\prime}\left(\frac{1}{\mathrm{p}}-\frac{1}{2}\right)}\|\phi\|_{W_{1}^{1, \mathrm{p}}}^{\mathrm{p}^{\prime}}+c(1+|t|)^{\mathrm{p}^{\prime}}|t|^{-\mathrm{p}^{\prime}\left(\frac{1}{\mathrm{p}}-\frac{1}{2}\right)}\left\|\phi * \varrho_{q}\right\|_{W_{1}^{1, \mathrm{p}}}^{\mathrm{p}^{\prime}} \\
& \leq \bar{c}(1+|t|)^{\mathrm{p}^{\prime}}|t|^{-\mathrm{p}^{\prime}\left(\frac{1}{\mathrm{p}}-\frac{1}{2}\right)}\|\phi\|_{W_{1}^{1, \mathrm{p}}}^{\mathrm{p}^{\prime}}
\end{aligned}
$$

$\log \mathrm{e}$

$$
\left\|U_{q}(t) \phi\right\|_{W_{1}^{1, \mathrm{p}^{\prime}}} \leq C(1+|t|)|t|^{-\left(\frac{1}{\mathrm{p}}-\frac{1}{2}\right)}\|\phi\|_{W_{1}^{1, \mathrm{p}}}
$$

Das equações (5.2), (5.4) e (5.5), obtemos (5.18), (5.19) e (5.20), respectivamente.

Para verificar que $t \mapsto U_{q}(t)$ é fortemente contínua, basta usar o Lema 3.4.1 e notar que

$$
x U_{q}(s) \phi-x U_{q}(t) \phi=U_{0}(s)(x-2 i s \partial) \phi-U_{0}(t)(x-2 i t \partial) \phi
$$




$$
\begin{aligned}
& +\operatorname{sign}(x)\left(U_{0}(s)(x-i s \partial)\left(\phi * \varrho_{q}\right)(|x|)-U_{0}(t)(x-i t \partial)\left(\phi * \varrho_{q}\right)(|x|)\right) \\
= & {\left[U_{0}(s)-U_{0}(t)\right](x-2 i s \partial) \phi(x)+U_{0}(t)[(x-2 i s \partial) \phi-(x-2 i t \partial) \phi(x)] } \\
& +\operatorname{sign}(x)\left[U_{0}(s)-U_{0}(t)\right](x-i s \partial)\left(\phi * \varrho_{q}\right)(|x|) \\
& -\operatorname{sign}(x) U_{0}(t)\left[(x-2 i s \partial)\left(\phi * \varrho_{q}\right)(|x|)-(x-2 i t \partial)\left(\phi * \varrho_{q}\right)(|x|)\right]
\end{aligned}
$$

$\operatorname{com} t, s \in \mathbb{R}-\{0\}\left(t, s \in \mathbb{R}\right.$, se $\left.^{\prime}=2\right)$.

Sejam $u_{j} \in C_{0}\left([0, T] ; H_{1}^{1}\right), j=1,2, \ldots$, onde $C_{0}$ é o espaço das funções contínuas de suporte compacto, tal que

$$
\left\|u_{j}-u\right\|_{L_{T}^{r^{\prime}} L_{x}^{\mathrm{p}}} \rightarrow 0, \text { quando } j \rightarrow \infty
$$

então, por (5.20),

$$
\left\|I\left(\cdot, u_{j}\right)-I(\cdot, u)\right\|_{L_{T}^{\infty} H_{1}^{1}} \rightarrow 0, \text { quando } j \rightarrow \infty
$$

e, pela propriedade de $C_{0}$-grupo de $U_{0}(t)$ sobre $H_{1}^{1}, I\left(\cdot, u_{j}\right) \in C\left([0, T] ; H_{1}^{1}\right)$. Portanto, $I(\cdot, u) \in$ $C\left([0, T] ; H_{1}^{1}\right)$.

Vamos estender também o Lema anterior para o espaço $W_{m}^{1, \mathrm{p}^{\prime}}(\mathbb{R})$.

Lema 5.2.5. Para qualquer $\mathrm{p}^{\prime}$ com $2 \leq \mathrm{p}^{\prime} \leq \infty$ e para qualquer $t \in \mathbb{R} \backslash\{0\}, U_{q}(t): W_{m}^{1, \mathrm{p}} \rightarrow W_{m}^{1, \mathrm{p}^{\prime}}$ é um um operador limitado com

$$
\left\|U_{q}(t) \phi\right\|_{W_{m}^{1, \mathrm{p}^{\prime}}} \leq c(1+|t|)|t|^{-\left(\frac{1}{\mathrm{p}}-\frac{1}{2}\right)}\|\phi\|_{W_{m}^{1, \mathrm{p}}}
$$

para toda $\phi \in W_{m}^{1, \mathrm{p}}$ e a aplicação $t \mapsto U_{q}(t)$ é fortemente contínua. Se $\mathrm{p}^{\prime}=2$, as afirmações vale para todo $t \in \mathbb{R}$. Mais ainda, para qualquer $\mathrm{p}^{\prime}$ e $r$ satisfazendo $2 \leq \mathrm{p}^{\prime}<\infty, 2 \leq r \leq \infty$ e $\frac{2}{r}=\frac{1}{2}-\frac{1}{\mathrm{p}^{\prime}}=\frac{1}{\mathrm{p}}-\frac{1}{2}, \mathrm{com}$

$$
I(t, u) \equiv \int_{0}^{t} U_{q}(t-s) u(s) d s,
$$

obtemos as seguintes desigualdades

$$
\begin{aligned}
& \left\|U_{q}(\cdot) \phi\right\|_{L_{T}^{r} W_{m}^{1, \mathrm{p}^{\prime}}} \leq c(1+T)\|\phi\|_{W_{m}^{1, \mathrm{p}}}, \quad \forall \phi \in W_{m}^{1, \mathrm{p}} \\
& \|I(\cdot, u)\|_{L_{T}^{r} W_{m}^{1, \mathrm{p}^{\prime}}} \leq c(1+T)\|u\|_{L_{T}^{r^{\prime} W_{m}^{1, \mathrm{p}}}}, \quad \forall u \in L^{r^{\prime}}\left(0, T ; W_{m}^{1, \mathrm{p}}\right) \\
& \|I(t, u)\|_{H_{m}^{1}} \leq c(1+T)\|u\|_{L_{T}^{r^{\prime} W_{m}^{1, \mathrm{p}}}}, \quad \forall u \in L^{r^{\prime}}\left(0, T ; W_{m}^{1, \mathrm{p}}\right),
\end{aligned}
$$

para todo $t \in[0, T] e \frac{1}{r}+\frac{1}{r^{\prime}}=1$. Mais ainda, para cada $u \in L^{r^{\prime}}\left(0, T ; W^{1, \mathrm{p}}\right)$, temos $I(\cdot, u) \in$ $C\left([0, T] ; H_{m}^{1}\right)$.

Demonstração: Análoga ao Lema 5.2.4.

Seguindo as demonstrações dos Lemas 5.2.3 e 5.2.5, respectivamente, percebemos que podemos obter propriedades para $U_{q}$ em $W_{m}^{2, \mathrm{p}}(\Omega)$ a seguir. 
Lema 5.2.6. Seja $2 \leq \mathrm{p}^{\prime} \leq \infty$. Para todo $t \in \mathbb{R}, U_{q}(t): W_{m}^{2, \mathrm{p}}(\Omega) \rightarrow W_{m}^{2, \mathrm{p}^{\prime}}(\Omega)$ é um operador limitado, com $\frac{1}{\mathrm{p}}+\frac{1}{\mathrm{p}^{\prime}}=1$. Além disso, para qualquer $t, s \in \mathbb{R}$ com $t \neq s$ e para toda $\phi \in W_{m}^{2, \mathrm{p}}(\Omega)$, temos

$$
(x+i t \partial)^{2} U_{q}(t-s) \phi(x)=U_{q}(t-s)(x+i s \partial)^{2} \phi(x) .
$$

Para $\mathrm{p}^{\prime}=2, U_{q}(t)$ é limitado para todo $t \in \mathbb{R}$ e a equação (5.21) vale para todo $t, s \in \mathbb{R}$.

Lema 5.2.7. Para qualquer $p^{\prime}$ com $2 \leq \mathrm{p}^{\prime} \leq \infty$ e para qualquer $t \in \mathbb{R} \backslash\{0\}, U_{q}(t): W_{m}^{2, \mathrm{p}}(\Omega) \rightarrow$ $W_{m}^{2, \mathrm{p}^{\prime}}(\Omega)$ é um um operador limitado com

$$
\left\|U_{q}(t) \phi\right\|_{W_{m}^{2, \mathrm{p}^{\prime}}(\Omega)} \leq c(1+|t|)^{2}|t|^{-\left(\frac{1}{\mathrm{p}}-\frac{1}{2}\right)}\|\phi\|_{W_{m}^{2, \mathrm{p}}(\Omega)},
$$

para toda $\phi \in W_{m}^{2, p}(\Omega)$ e a aplicação $t \mapsto U_{q}(t)$ é fortemente contínua. Se $\mathrm{p}^{\prime}=2$, as afirmações vale para todo $t \in \mathbb{R}$. Mais ainda, para qualquer $\mathrm{p}^{\prime}$ e $r$ satisfazendo $2 \leq \mathrm{p}^{\prime}<\infty, 2 \leq r \leq \infty$ e $\frac{2}{r}=\frac{1}{2}-\frac{1}{\mathrm{p}^{\prime}}=\frac{1}{\mathrm{p}}-\frac{1}{2}, \mathrm{com}$

$$
I(t, u) \equiv \int_{0}^{t} U_{q}(t-s) u(s) d s,
$$

obtemos as seguintes desigualdades

$$
\begin{aligned}
\left\|U_{q}(\cdot) \phi\right\|_{L_{T}^{r} W_{m}^{2, \mathrm{p}^{\prime}}(\Omega)} & \leq c(1+T)^{2}\|\phi\|_{W_{m}^{2, \mathrm{p}}(\Omega)}, \quad \forall \phi \in W_{m}^{2, \mathrm{p}}(\Omega) \\
\|I(\cdot, u)\|_{L_{T}^{r} W_{m}^{2, \mathrm{p}^{\prime}}(\Omega)} & \leq c(1+T)^{2}\|u\|_{L_{T}^{r^{\prime}} W_{m}^{2, \mathrm{p}}(\Omega)}, \quad \forall u \in L^{r^{\prime}}\left(0, T ; W_{m}^{2, \mathrm{p}}(\Omega)\right) \\
\|I(t, u)\|_{H_{2}^{2}(\Omega)} & \leq c(1+T)^{2}\|u\|_{L_{T}^{r^{\prime}} W_{2}^{2, \mathrm{p}}(\Omega)}, \quad \forall u \in L^{r^{\prime}}\left(0, T ; W_{m}^{2, \mathrm{p}}(\Omega)\right),
\end{aligned}
$$

para todo $t \in[0, T] e \frac{1}{r}+\frac{1}{r^{\prime}}=1$. Mais ainda, para cada $u \in L^{r^{\prime}}\left(0, T ; W_{m}^{2, \mathrm{p}}(\Omega)\right)$, temos $I(\cdot, u) \in$ $C\left([0, T] ; H_{m}^{2}(\Omega)\right)$.

\subsection{Propriedades das soluções no espectro contínuo}

Consideremos o seguinte problema de Cauchy

$$
\left\{\begin{array}{l}
i \frac{\partial u}{\partial t}=H_{q} u-\lambda|u|^{p} u, \quad(x, t) \in \mathbb{R} \times \mathbb{R}, \\
u(0)=u_{0} \in H_{m}^{1}(\mathbb{R}) .
\end{array}\right.
$$

Para $u=u(x, t)$ solução de (5.26), usando o Teorema Espectral (veja página 26), temos formalmente a decomposição $u(x, t)=u_{p}(t) \psi_{0}(x)+u_{c}(x, t)$. Recordemos que $\psi_{0}$ é a autofunção associada ao autovalor negativo $E_{0}$. Se aplicarmos as projeções $P_{c}$ e $P_{p}$ em (5.26), podemos reescrevê-lo como o sistema

$$
\left\{\begin{array}{l}
i \frac{\partial u_{p}}{\partial t}=E_{0} u_{p}-\lambda\left\langle\left|u_{p} \psi_{0}+u_{c}\right|^{p}\left(u_{p} \psi_{0}+u_{c}\right), \psi_{0}\right\rangle \\
i \frac{\partial u_{c}}{\partial t}=H_{q} u_{c}-\lambda P_{c}\left(\left|u_{p} \psi_{0}+u_{c}\right|^{p}\left(u_{p} \psi_{0}+u_{c}\right)\right) \\
u(0)=u_{p}(0) \psi_{0}+u_{c}(0) \equiv u_{0} \in H_{m}^{1}(\mathbb{R})
\end{array}\right.
$$


onde $u_{p}=u_{p}(t) \in \mathbb{C}, u_{c}=u_{c}(t) \in \mathscr{R}\left(P_{c}\right)$. Note que se $u$ é solução de (5.26) é tal que $u \in \mathscr{R}\left(P_{c}\right)$, então $u_{p}(t)=0$, para todo $t$. Logo podemos escrever (5.26)-(5.27) na forma da equação integral

$$
u_{c}(t)=U_{q}(t) u_{c}(0)+i \lambda \int_{0}^{t} U_{q}(t-s) P_{c}\left(\left|u_{c}(s)\right|^{p}\left(u_{c}(s)\right)\right) d s
$$

onde $U_{q}(t)=e^{-i t H_{q}}-e^{-i t \frac{q^{2}}{2}} P_{p} \equiv e^{-i t H_{q}}$ e $u_{c}(0)=u_{0} \in \mathscr{R}\left(P_{c}\right)$.

Observação 5.3.1. Usando (5.28), obtemos imediatamente que $u_{c} \in \mathscr{R}\left(P_{c}\right)$. De fato, como $U_{q}(t) f \in \mathscr{R}\left(P_{c}\right)$, para $f \in \mathscr{R}\left(P_{c}\right)$, obtemos da continuidade do projetor $P_{c}$ que $P_{c} u(t)=U_{q}(t) u(0)+$ $i \lambda \int_{0}^{t} U_{q}(t-s) P_{c}\left(\left|u_{c}(s)\right|^{p}\left(u_{c}(s)\right)\right) \equiv u(t)$

Teorema 5.3.1. Para qualquer $u_{0} \in H_{1}^{1} \cap \mathscr{R}\left(P_{c}\right)$, existe $T=T\left(\left\|u_{0}\right\|_{H_{1}^{1}}, p, \lambda\right)>0$ e uma única solução $u_{c}$ de (5.28) em $[0, T]$ com

$$
u_{c} \in C\left([0, T] ; H_{1}^{1} \cap \mathscr{R}\left(P_{c}\right)\right) .
$$

Além disso, $T=\infty$ ou $T<\infty$ e $\lim _{t \rightarrow T^{-}}\left\|u_{c}(t)\right\|_{H_{1}^{1}}=\infty$.

Demonstração: Seja $T>0$. Denotamos $X=C\left([0, T] ; H_{1}^{1}\right)$ com norma \|\|$_{L_{T}^{\infty} H_{1}^{1}}$.

Seja $u_{0} \in H_{1}^{1} \cap \mathscr{R}\left(P_{c}\right)$ e defina para $u_{c} \in X$, pela fórmula de Duhamel,

$$
G u_{c}(t) \equiv U_{q}(t) u_{0}-i \lambda \int_{0}^{t} U_{q}(t-s) P_{c}\left(\left|u_{c}(s)\right|^{p} u_{c}(s)\right) d s .
$$

Primeiramente, note que para toda $w \in H_{1}^{1}$,

$$
\left\|P_{c}(w)\right\|_{H_{1}^{1}}=\left\|w-P_{p}(w)\right\|_{H_{1}^{1}} \leq\|w\|_{H_{1}^{1}}+\left|\left\langle w, \psi_{0}\right\rangle\right|\left\|\psi_{0}\right\|_{H_{1}^{1}} \leq C\|w\|_{H_{1}^{1}} .
$$

Além disso,

$$
\begin{aligned}
\int_{0}^{T}\left\|P_{c}\left(\left|u_{c}(s)\right|^{p} u_{c}(s)\right)\right\|_{H_{1}^{1}} d s & \leq \int_{0}^{T}\left\|\left|u_{c}(s)\right|^{p} u_{c}(s)\right\|_{H_{1}^{1}} d s+\int_{0}^{T}\left|\left\langle\left|u_{c}(s)\right|^{p} u_{c}(s), \psi_{0}\right\rangle\right|\left\|\psi_{0}\right\|_{H_{1}^{1}} d s \\
& \leq \int_{0}^{T}\left\|\left|u_{c}(s)\right|^{p} u_{c}(s)\right\|_{H_{1}^{1}} d s+c \int_{0}^{T}\left\|\left|u_{c}(s)\right|^{p} u_{c}(s)\right\|_{2}, d s \\
& \leq C\left\|\left|u_{c}\right|^{p} u_{c}\right\|_{L_{T}^{1} H_{1}^{1}},
\end{aligned}
$$

ou seja, $P_{c}\left(\left|u_{c}\right|^{p} u_{c}\right) \in L^{1}\left(0, T ; H_{1}^{1}\right)$. Chame $w=P_{c}\left(\left|u_{c}\right|^{p} u_{c}\right)$. Escreveremos

$$
I(\cdot, w)=\int_{0}^{t} U_{q}(t-s) w(s) d s .
$$

Pelo Lema 5.2.4, obtemos do fato que $w \in L^{1}\left(0, T ; H_{1}^{1}\right)$

$$
\|I(\cdot, w)\|_{X} \leq c(1+T)\|w\|_{L_{T}^{1} H_{1}^{1}} \leq C(1+T)\left\|\left|u_{c}\right|^{p} u_{c}\right\|_{L_{T}^{1} H_{1}^{1}}
$$

Construiremos uma solução $u_{c}(t)$ de (5.28) em $X$ utilizando o método de contração para 
$\operatorname{algum} T>0$.

Sejam $u_{c}, v_{c} \in X$, então

$$
\begin{aligned}
\left\|G u_{c}-G v_{c}\right\|_{X} & =|\lambda|\left\|\int_{0}^{t} U_{q}(t-s) P_{c}\left(\left|u_{c}(s)\right|^{p} u_{c}(s)-\left|v_{c}(s)\right|^{p} v_{c}(s)\right) d s\right\|_{X} \\
& =|\lambda| \| I\left(\cdot, P_{c}\left(\left|u_{c}\right|^{p} u_{c}-\left|v_{c}\right|^{p} v_{c}\right) \|_{X}\right. \\
& \leq c(1+T)|\lambda|\left\|\left|u_{c}\right|^{p} u_{c}-\left|v_{c}\right|^{p} v_{c}\right\|_{L_{T}^{1} H_{1}^{1}} \\
& =c(1+T) T|\lambda|\left\|\left|u_{c}\right|^{p} u_{c}-\left|v_{c}\right|^{p} v_{c}\right\|_{L_{T}^{\infty} H_{1}^{1}} \\
& =c(1+T) T|\lambda|\left\|\left|\left\|\left|u_{c}\right|^{p} u_{c}-\left|v_{c}\right|^{p} v_{c}\right\|_{H_{1}^{1}} \|_{L_{T}^{\infty}} .\right.\right.
\end{aligned}
$$

Note que

$$
\begin{aligned}
\left\|\left|u_{c}(t)\right|^{p} u_{c}(t)-\left|v_{c}(t)\right|^{p} v_{c}(t)\right\|_{H_{1}^{1}}^{2}= & \left\|\left|u_{c}(t)\right|^{p} u_{c}(t)-\left|v_{c}(t)\right|^{p} v_{c}(t)\right\|_{H_{1}}^{2} \\
& +\sum_{\alpha=0}^{1}\left\|x^{\alpha}\left(\left|u_{c}(t)\right|^{p} u_{c}(t)-\left|v_{c}(t)\right|^{p} v_{c}(t)\right)\right\|_{2}^{2} .
\end{aligned}
$$

Da desigualdade do valor médio, segue que

$$
\left.|| u_{c}\right|^{p} u_{c}-\left|v_{c}\right|^{p} v_{c}\left|\leq C\left(\left|u_{c}\right|^{p}+\left|v_{c}\right|^{p}\right)\right| u_{c}-v_{c} \mid
$$

e

$$
\begin{aligned}
\left.|| u_{c}\right|^{p} \frac{\partial}{\partial x} u_{c}-\left|v_{c}\right|^{p} \frac{\partial}{\partial x} v_{c} \mid & \leq\left.|| u_{c}\right|^{p} \frac{\partial}{\partial x} u_{c}-\left|v_{c}\right|^{p} \frac{\partial}{\partial x} u_{c}|+|\left|v_{c}\right|^{p} \frac{\partial}{\partial x} u_{c}-\left|v_{c}\right|^{p} \frac{\partial}{\partial x} v_{c} \mid \\
& \leq\left.|| u_{c}\right|^{p-1} u_{c}-\left.\left|v_{c}\right|^{p-1} v_{c}|| \frac{\partial}{\partial x} u_{c}|+| v_{c}\right|^{p}\left|\frac{\partial}{\partial x} u_{c}-\frac{\partial}{\partial x} v_{c}\right| \\
& \leq C\left(\left|u_{c}\right|^{p-1}+\left|v_{c}\right|^{p-1}\right)\left|u_{c}-v_{c}\right|\left|\frac{\partial}{\partial x} u_{c}\right|+C\left|v_{c}\right|^{p}\left|\frac{\partial}{\partial x}\left(u_{c}-v_{c}\right)\right|
\end{aligned}
$$

logo, da primeira parcela, obtemos

$$
\left\|\left|u_{c}(t)\right|^{p} u_{c}(t)-\left|v_{c}(t)\right|^{p} v_{c}(t)\right\|_{H_{1}}^{2} \leq C\left(\left\|u_{c}(t)\right\|_{H_{1}}^{p}+\left\|v_{c}(t)\right\|_{H_{1}}^{p}\right)^{2}\left\|u_{c}(t)-v_{c}(t)\right\|_{H_{1}}^{2},
$$

e da segunda,

$$
\begin{aligned}
\left\|x^{\alpha}\left(\left|u_{c}(t)\right|^{p} u_{c}(t)-\left|v_{c}(t)\right|^{p} v_{c}(t)\right)\right\|_{2}^{2} & \leq C\left\|\left(\left|u_{c}(t)\right|^{p}+\left|v_{c}(t)\right|^{p}\right)\left|x^{\alpha}\left(u_{c}(t)-v_{c}(t)\right)\right|\right\|_{2}^{2} \\
& \leq C\left(\left\|u_{c}(t)\right\|_{H_{1}}^{p}+\left\|v_{c}(t)\right\|_{H_{1}}^{p}\right)^{2}\left\|x^{\alpha}\left(u_{c}(t)-v_{c}(t)\right)\right\|_{2}^{2},
\end{aligned}
$$

então

$$
\left\|\left|u_{c}(t)\right|^{p} u_{c}(t)-\left|v_{c}(t)\right|^{p} v_{c}(t)\right\|_{H_{1}^{1}}^{2} \leq C\left(\left\|u_{c}(t)\right\|_{H_{1}}^{p}+\left\|v_{c}(t)\right\|_{H_{1}}^{p}\right)^{2}\left\|u_{c}(t)-v_{c}(t)\right\|_{H_{1}^{1}}^{2}
$$


mas $H_{1}^{1} \subset H^{1}$, assim

$$
\left\|\left|u_{c}(t)\right|^{p} u_{c}(t)-\left|v_{c}(t)\right|^{p} v_{c}(t)\right\|_{H_{1}^{1}} \leq C\left(\left\|u_{c}(t)\right\|_{H_{1}^{1}}^{p}+\left\|v_{c}(t)\right\|_{H_{1}^{1}}^{p}\right)\left\|u_{c}(t)-v_{c}(t)\right\|_{H_{1}^{1}},
$$

$\log 0$

$$
\begin{aligned}
\left\|G u_{c}-G v_{c}\right\|_{X} & \leq c(1+T) T|\lambda|\left\|\left(\left\|u_{c}\right\|_{H_{1}^{1}}^{p}+\left\|v_{c}\right\|_{H_{1}^{1}}^{p}\right)\right\| u_{c}-v_{c}\left\|_{H_{1}^{1}}\right\|_{L^{\infty}(0, T)} \\
& \leq c(1+T) T|\lambda|\left(\left\|u_{c}\right\|_{X}^{p}+\left\|v_{c}\right\|_{X}^{p}\right)\left\|u_{c}-v_{c}\right\|_{X},
\end{aligned}
$$

portanto, obtemos

$$
\left\|G u_{c}-G v_{c}\right\|_{X} \leq c(1+T) T|\lambda|\left(\left\|u_{c}\right\|_{X}^{p}+\left\|v_{c}\right\|_{X}^{p}\right)\left\|u_{c}-v_{c}\right\|_{X} \cdot
$$

Agora estimaremos $G u_{c}$. Da equação (5.21), com $\mathrm{p}=2$ ( $\mathrm{p}$ do espaço de Sobolev), para algum $\bar{c}>0$

$$
\left\|U_{q}(t) u_{0}\right\|_{H_{1}^{1}} \leq \bar{c}(1+|t|)\left\|u_{0}\right\|_{H_{1}^{1}}, \quad \text { para } t \in[0, T]
$$

então, para $T$ pequeno,

$$
\left\|U_{q}(\cdot) u_{0}\right\|_{X} \leq \bar{c}(1+T)\left\|u_{0}\right\|_{H_{1}^{1}}
$$

e, da equação (5.30), $\left\|I\left(\cdot, P_{c}\left(\left|u_{c}\right|^{p} u_{c}\right)\right)\right\|_{X} \leq c(1+T)\left\|\left|u_{c}\right|^{p} u_{c}\right\|_{L_{T}^{1} H_{1}^{1}}$. Veja que $\left\|\left|u_{c}\right|^{p} u_{c}\right\|_{H_{1}^{1}}^{2}<\infty$.

De fato,

$$
\left\|\left|u_{c}\right|^{p} u_{c}\right\|_{H_{1}^{1}}^{2} \leq c\left(\left\|\left|u_{c}\right|^{p} u_{c}\right\|_{H_{1}}^{2}+\left\|x\left|u_{c}\right|^{p} u_{c}\right\|_{2}^{2}\right) \leq c_{1}\left(\left\|u_{c}\right\|_{H_{1}}^{2(p+1)}+\left\|x\left|u_{c}\right|^{p} u_{c}\right\|_{2}^{2}\right),
$$

$\mathrm{e}$

$$
\left\|x\left|u_{c}\right|^{p} u_{c}\right\|_{2}^{2} \leq\left\|u_{c}\right\|_{\infty}^{2 p}\left\|x u_{c}\right\|_{2}^{2} \leq\left\|u_{c}\right\|_{H_{1}}^{2 p}\left\|u_{c}\right\|_{H_{1}^{1}}^{2} \leq\left\|u_{c}\right\|_{H_{1}^{1}}^{2(p+1)}
$$

$\operatorname{assim}\left\|\left|u_{c}\right|^{p} u_{c}\right\|_{H_{1}^{1}} \leq\left\|u_{c}\right\|_{H_{1}^{1}}^{p+1}$. Logo, de (5.30),

$$
\left\|I\left(\cdot, P_{c}\left(\left|u_{c}\right|^{p} u_{c}\right)\right)\right\|_{X} \leq c(1+T) T\left\|u_{c}\right\|_{L_{T}^{\infty} H_{1}^{1}}^{p}\left\|u_{c}\right\|_{L_{T}^{\infty} H_{1}^{1}} \leq c(1+T) T\left\|u_{c}\right\|_{X}^{p}\left\|u_{c}\right\|_{X} .
$$

Então, para $T$ pequeno,

$$
\left\|G u_{c}\right\|_{X} \leq c_{1}(1+T)\left(\left\|u_{0}\right\|_{H_{1}^{1}}+|\lambda| T\left\|u_{c}\right\|_{X}^{p}\left\|u_{c}\right\|_{X}\right)
$$

onde $c_{1}=\max \{c, \bar{c}\}$.

Seja $K$ qualquer número tal que $\left\|u_{0}\right\|_{H_{1}^{1}} \leq K$. Com $b>0$ fixo, consideremos

$$
B_{K, T}=\left\{u_{c} \in X ;\left\|u_{c}\right\|_{X} \leq\left(c_{1}+b\right) K+b\right\}
$$

Veremos que $G: B_{K, T} \rightarrow B_{K, T}$ é uma contração para um $T$ pequeno. Seja $u_{c} \in B_{K, T}$. Então,

$$
\left\|G u_{c}\right\|_{X} \leq c_{1}(1+T)\left[K+|\lambda| T\left(\left(c_{1}+b\right) K+b\right)^{p}\left(\left(c_{1}+b\right) K+b\right)\right]
$$




$$
\leq(1+T)\left[\frac{c_{1} K}{\left(c_{1}+b\right) K+b}+c_{1}|\lambda| T\left(\left(c_{1}+b\right) K+b\right)^{p}\right]\left(\left(c_{1}+b\right) K+b\right) .
$$

Queremos que

$$
\frac{c_{1} K}{\left(c_{1}+b\right) K+b}+c_{1}|\lambda| T\left(\left(c_{1}+b\right) K+b\right)^{p}<\frac{1}{1+T} .
$$

Seja $b>0$ (a ser escolhido) tal que

$$
\frac{c_{1} K}{\left(c_{1}+b\right) K+b}<\frac{1}{2} \frac{1}{1+T} \Leftrightarrow T<\frac{b(K+1)-c_{1} K}{2 c_{1} K}
$$

e $T$ tal que $T(1+T)<\frac{1}{2 c_{1}|\lambda|\left(\left(c_{1}+b\right) K+b\right)^{p}}$. Logo escolhamos $b$ tal que $b(K+1)>c_{1} K$. Assim, com essa escolha de $T$

$$
\left\|G u_{c}\right\|_{X} \leq\left(c_{1}+b\right) K+b
$$

isto é, $G$ é uma aplicação de $B_{K, T}$ em $B_{K, T}$; mais ainda, da equação (5.31),

$$
\begin{aligned}
\left\|G u_{c}-G v_{c}\right\|_{B_{K, T}} & \leq c(1+T) T|\lambda|\left(\left\|u_{c}\right\|_{X}^{p}+\left\|v_{c}\right\|_{X}^{p}\right)\left\|u_{c}-v_{c}\right\|_{X} \\
& \leq c(1+T) T|\lambda|\left(\left(\left(c_{1}+b\right) K+b\right)^{p}+\left(\left(c_{1}+b\right) K+b\right)^{p}\right)\left\|u_{c}-v_{c}\right\|_{B_{K, T}} \\
& \leq 2 c_{1} T(1+T)\left[\left(\left(c_{1}+b\right) K+b\right)^{p}\right]\left\|u_{c}-v_{c}\right\|_{B_{K, T}} \\
& <a\left\|u_{c}-v_{c}\right\|_{B_{K, T}}
\end{aligned}
$$

onde $a \in(0,1)$, pela escolha de $T$. Logo, $G$ é uma contração de $B_{K, T}$ em $B_{K, T}$, para $T=$ $T_{K}\left(\left\|u_{0}\right\|_{H_{1}^{1}}\right)>0$ suficientemente pequeno. Então, existe uma única $u \in B_{K, T}$ tal que $G u_{c}=u_{c}$. Logo, $u_{c}$ satisfaz a equação de Duhamel (5.29), em $\left[0, T_{K}\right]$. Como para cada $K>0, T_{K}$ pode ser escolhido uniformemente para $u_{0}$ na bola em $H_{1}^{1}$ de raio $K$ e centro 0 , concluímos que a solução $u_{c}$ se estende unicamente para algum intervalo grande $\left[0, T_{\max }\right)$ tal que

$$
u_{c} \in C\left(\left[0, T_{\max }\right) ; H_{1}^{1}\right)
$$

A seguir veremos que $T_{\max }=\infty$ ou $T_{\max }<\infty$ e $\lim _{t \rightarrow T_{\max }^{-}}\left\|u_{c}\right\|_{H_{1}^{1}}=\infty$.

Inicialmente, pelas imersões de Sobolev temos

- $H_{m}^{k} \subset H^{k} \subset L^{2}$,

- $H_{m}^{k} \subset H^{k} \subset L^{\infty}$, para $k>\frac{1}{2}$,

logo, $H_{m}^{k} \subset L^{2} \cap L^{\infty}$, para $k>\frac{1}{2}$. Assim pela desigualdade de Hölder generalizada $H_{m}^{k} \subset L^{\tilde{p}}$, para todo $2 \leq \widetilde{p} \leq \infty$. Em particular, $H_{1}^{1} \subset L^{\widetilde{p}}$, para todo $2 \leq \widetilde{p} \leq \infty$.

Suponhamos que $T_{\max }<\infty, \lim _{t \rightarrow T_{\max }^{-}}\left\|u_{c}\right\|_{H_{1}^{1}}<\infty$ e $\left\|u_{c}(t)\right\|_{\infty}<d_{0}$, para $0<t<T_{\max }$. Queremos mostrar que $\left\|u_{c}(t)\right\|_{H_{1}^{1}} \leq d_{1}$ em [0,T $\left.T_{\max }\right)$, para alguma constante $d_{1}$, o que contradiz a definição de $T_{\max }$. Seja $0<t<T_{\max }$. Das equações (5.22) e (5.18), temos

$$
\left\|u_{c}(t)\right\|_{H_{1}^{1}} \leq\left\|U_{q}(t) u_{0}\right\|_{H_{1}^{1}}+|\lambda| \int_{0}^{t}\left\|U_{q}(t-s) P_{c}\left(\left|u_{c}(s)\right|^{p} u_{c}(s)\right)\right\|_{H_{1}^{1}} d s
$$




$$
\leq c(1+t)\left\|u_{0}\right\|_{H_{1}^{1}}+c|\lambda|(1+t) \int_{0}^{t}\left\|\left|u_{c}(s)\right|^{p} u_{c}(s)\right\|_{H_{1}^{1}} d s
$$

mas, sendo $\left\|u_{c}(t)\right\|_{\infty}<d_{0}$, da desigualdade de Kato-Ponce, temos

$$
\begin{aligned}
\left\|\left|u_{c}(s)\right|^{p} u_{c}(s)\right\|_{H_{1}^{1}} & \equiv\left\|\left|u_{c}(s)\right|^{p} u_{c}(s)\right\|_{H^{1}}+\left\|x\left|u_{c}(s)\right|^{p} u_{c}(s)\right\|_{2} \\
& =\left\|\left(1-\partial_{x}^{2}\right)^{1 / 2}\left(\left|u_{c}(s)\right|^{p} u_{c}(s)\right)\right\|_{2}+\left\|x\left|u_{c}(s)\right|^{p} u_{c}(s)\right\|_{2} \\
& \leq 2\left\|u_{c}(s)\right\|_{\infty}^{p}\left\|u_{c}(s)\right\|_{H^{1}}+\left\|u_{c}(s)\right\|_{\infty}^{p}\left\|x u_{c}(s)\right\|_{2} \\
& \leq c d_{0}^{p}\left\|u_{c}(s)\right\|_{H_{1}^{1}}
\end{aligned}
$$

$\log 0$

$$
\begin{aligned}
\left\|u_{c}(t)\right\|_{H_{1}^{1}} & \leq c(1+t)\left\|u_{0}\right\|_{H_{1}^{1}}+c d_{0}^{p}|\lambda|(1+t) \int_{0}^{t}\left\|u_{c}(s)\right\|_{H_{1}^{1}} d s \\
& \leq c\left(1+T_{\max }\right)\left\|u_{0}\right\|_{H_{1}^{1}}+c d_{0}^{p}|\lambda|\left(1+T_{\max }\right) \int_{0}^{t}\left\|u_{c}(s)\right\|_{H_{1}^{1}} d s
\end{aligned}
$$

pela desigualdade de Gronwall

$$
\begin{aligned}
\left\|u_{c}(t)\right\|_{H_{1}^{1}} & \leq c\left(1+T_{\max }\right)\left\|u_{0}\right\|_{H_{1}^{1}} \exp \left(\int_{0}^{t} c d_{0}^{p}|\lambda|\left(1+T_{\max }\right) d s\right) \\
& \leq K c\left(1+T_{\max }\right) \exp \left(c d_{0}^{p}|\lambda|\left(1+T_{\max }\right) T_{\max }\right)
\end{aligned}
$$

assim basta considerar $d_{1}=K c\left(1+T_{\max }\right) \exp \left(c d^{p}|\lambda|\left(1+T_{\max }\right) T_{\max }\right)$. Isto finaliza a demonstração do teorema.

Observação 5.3.2. Pela demonstração acima, concluímos: se $\left\|u_{0}\right\|_{H_{1}^{1}} \ll \varepsilon$, então $\left\|u_{c}\right\|_{H_{1}^{1}} \ll C \varepsilon$, $C>0$, para todo $t \in[0, T]$.

Corolário 5.3.1.1. Seja $m \geq 1$. Então, para qualquer $u_{0} \in H_{m}^{1} \cap \mathscr{R}\left(P_{c}\right)$, existe $T=T\left(\left\|u_{0}\right\|_{H_{m}^{1}}\right)>0$ e uma única solução u de (5.28) em $[0, T]$ com

$$
u \in C\left([0, T] ; H_{m}^{1} \cap \mathscr{R}\left(P_{c}\right)\right)
$$

Além disso, $T=\infty$ ou $T<\infty$ e $\lim _{t \rightarrow T^{-}}\|u(t)\|_{H_{m}^{1}}=\infty$.

Demonstração: Seja $u_{0} \in H_{m}^{1} \cap \mathscr{R}\left(P_{c}\right)$. Então dos Lemas 5.2.3 e 5.2.5 e da demonstração do Teorema 5.3.1, vemos que $T$ pode ser escolhido como no caso de $m=1$ e assim o teorema do ponto fixo implica o desejado.

De maneira similar podemos mostrar:

Teorema 5.3.2. Seja $m \leq 2$. Então, para qualquer $u_{0} \in H_{1}^{1}(\mathbb{R}) \cap H_{m}^{2}(\Omega) \cap \mathscr{R}\left(P_{c}\right)$, existe $T=$ $T\left(\left\|u_{0}\right\|_{H_{1}^{1}(\mathbb{R}) \cap H_{m}^{2}(\Omega)}\right)>0$ e uma única solução $u_{c}$ de (5.28) em $[0, T)$ com

$$
u_{c} \in C\left([0, T] ; H_{1}^{1}(\mathbb{R}) \cap H_{m}^{2}(\Omega) \cap \mathscr{R}\left(P_{c}\right)\right)
$$


Além disso, $T=\infty$ ou $T<\infty$ e $\lim _{t \rightarrow T^{-}}\left\|u_{c}(t)\right\|_{H_{1}^{1}(\mathbb{R}) \cap H_{m}^{2}(\Omega)}=\infty$.

Observação 5.3.3. Novamente, se $\left\|u_{o}\right\|_{H_{1}^{1}(\mathbb{R}) \cap H_{m}^{2}(\Omega)} \ll \varepsilon$, então $\left\|u_{c}\right\|_{H_{1}^{1}(\mathbb{R}) \cap H_{m}^{2}(\Omega)} \ll C \varepsilon, C>0$, para todo $t \in[0, T]$. 


\section{Aproximação à Variedade Centro e convergência para uma órbita}

Nesse capítulo, iniciamos na Seção 6.1 mostrando uma estimativa dispersiva para $e^{-i t H_{q}} P_{c} f$ em $L_{-s-2 \beta}^{2}$, com $s>1$ e $0 \leq \beta \leq 1$. Tal resultado será aplicado tanto no Teorema 6.2.1 como também no principal resultado do Capítulo 7 onde mudaremos nossa não linearidade para ser não polinomial como forma de obtermos uma aplicação.

Em seguida, usando o que apresentamos no Capítulo 5, demonstraremos que soluções começando próximas da variedade centro $\mathcal{W}_{\mu}^{p}$ vão se aproximar de $\mathcal{W}_{\mu}^{p}$ ( veja Figura 6.2). A partir disso, finalizamos com a Seção 6.3 mostrando que toda solução de (3.1) se aproxima de uma órbita particular na variedade centro local $\mathcal{W}_{\mu}^{p}$.

\subsection{A Estimativa $L_{s+2 \beta}^{2}-L_{-s-2 \beta}^{2}$}

Antes de mostrarmos o principal resultado desse capítulo, vamos obter uma estimativa para o grupo $e^{-i t H_{q}}$ entre os espaços $L_{s+2 \beta}^{2}$ e $L_{-s-2 \beta}^{2}$. De fato, na Seção 3.2 vimos que para $q<0$

$$
e^{-i t H_{q}}=\frac{1}{2 \pi} \int_{0}^{\infty} e^{-i t \frac{\lambda^{2}}{2}}\left(e_{+}(x, \lambda) \overline{e_{+}(y, \lambda)}+e_{-}(x, \lambda) \overline{e_{-}(y, \lambda)}\right) d \lambda+e^{\frac{1}{2} i t \frac{q^{2}}{2}} P_{p}
$$

com $P_{p} f=\left\langle f, \psi_{0}\right\rangle f$. No caso $q \geq 0$, o termo $e^{\frac{1}{2} i t \frac{q^{2}}{2}} P_{p}$ na fórmula (6.1) não aparece. Além disso, a projeção espectral contínua, $P_{c}$, é dada então como

$$
P_{c} f(x)=\frac{1}{2 \pi} \int_{\mathbb{R}} \int_{0}^{\infty}\left(e_{+}(x, \lambda) \overline{e_{+}(y, \lambda)}+e_{-}(x, \lambda) \overline{e_{-}(y, \lambda)}\right) f(y) d \lambda d y
$$

Podemos representar a expressão $e^{-i t H_{q}} P_{c}$ como

$$
e^{-i t H_{q}} P_{c} f(x)=\frac{1}{2 \pi} \int_{\mathbb{R}} \int_{0}^{\infty} e^{-i t \frac{\lambda^{2}}{2}}\left(e_{+}(x, \lambda) \overline{e_{+}(y, \lambda)}+e_{-}(x, \lambda) \overline{e_{-}(y, \lambda)}\right) f(y) d \lambda d y .
$$


Definindo $\Psi_{+}(y, \lambda)=\frac{1}{\sqrt{2 \pi}}\left\{\begin{array}{ll}e_{+}(y, \lambda), & \lambda \geq 0 \\ e_{-}(y,-\lambda), & \lambda<0\end{array}\right.$, obtemos

$$
\begin{aligned}
\frac{1}{2 \pi} \int_{\mathbb{R}} \int_{0}^{\infty} e_{-}(x, \lambda) \overline{e_{-}(y, \lambda)} f(y) d \lambda d y & =\frac{1}{2 \pi} \int_{\mathbb{R}} \int_{-\infty}^{0} e_{-}(x,-\lambda) \overline{e_{-}(y,-\lambda)} f(y) d \lambda d y \\
& =\int_{\mathbb{R}} \int_{-\infty}^{0} \Psi_{+}(x, \lambda) \overline{\Psi_{+}(y, \lambda)} f(y) d \lambda d y
\end{aligned}
$$

então

$$
P_{c} f(x)=\int_{\mathbb{R}} \int_{\mathbb{R}} \Psi_{+}(x, \lambda) \overline{\Psi_{+}(y, \lambda)} f(y) d \lambda d y,
$$

portanto,

$$
e^{-i t H_{q}} P_{c} f(x)=\int_{\mathbb{R}} \int_{\mathbb{R}} e^{-i t \frac{\lambda^{2}}{2}} \Psi_{+}(x, \lambda) \overline{\Psi_{+}(y, \lambda)} f(y) d \lambda d y .
$$

Definimos $\Psi_{-}(y,-k)=\overline{\Psi_{+}(y,-k)}$.

Teorema 6.1.1. Para todo $s>1$ e $0 \leq \beta \leq 1$, existe uma constante $C$ independente de $f$ e $t$ tal que

$$
\left\|e^{-i t H_{q}} P_{c} f\right\|_{L_{-s-2 \beta}^{2}} \leq C(1+|t|)^{-\frac{1}{2}-\beta}\|f\|_{L_{s+2 \beta}^{2}}, \quad \forall t \in \mathbb{R},
$$

para toda $f \in L_{s+2 \beta}^{2}$.

Demonstração: Para qualquer $\phi \in L^{1}(\mathbb{R}) \cap L^{2}(\mathbb{R})$,

$$
e^{-i t H_{q}} P_{c} \phi(x)=\int_{\mathbb{R}} \Phi_{t}(x, y) \phi(y) d y,
$$

onde

$$
\Phi_{t}(x, y)=\int_{\mathbb{R}} e^{-i \frac{k^{2}}{2} t} \Psi_{+}(x, k) \overline{\Psi_{+}(y, k)} d k .
$$

Sabemos do Lema 3.4.1 que

$$
\begin{array}{ll}
\left|\Phi_{t}(x, y)\right| \leq C \frac{1}{\sqrt{|t|}}, \quad & \text { para } \phi \in L^{1}, \forall t \neq 0, x, y \in \mathbb{R} \\
\left|\Phi_{t}(x, y)\right| \leq C, \quad \text { para } \phi \in L^{2}, \forall t, x, y \in \mathbb{R}
\end{array}
$$

então

$$
(1+|t|)^{\frac{1}{2}}\left|\Phi_{t}(x, y)\right| \leq c\left(1+|t|^{\frac{1}{2}}\right)\left|\Phi_{t}(x, y)\right| \leq C,
$$

ou seja,

$$
\left|\Phi_{t}(x, y)\right| \leq \frac{C}{(1+|t|)^{\frac{1}{2}}}
$$

Mostraremos a seguir que

$$
(1+|x|)^{-2}\left|\Phi_{t}(x, y)\right|(1+|y|)^{-2} \leq C \frac{1}{|t|^{3 / 2}}, \quad \forall t \neq 0 .
$$


Seja $\chi_{1} \in C_{0}^{\infty}(\mathbb{R})$ tal que, $\chi_{1}\left(\frac{k^{2}}{2}\right)=1$ para $|k| \leq \sqrt{2}$ e $\chi_{1}\left(\frac{k^{2}}{2}\right)=0$ para $|k| \geq 2$ e denotamos $\chi_{2}:=1-\chi_{1}$. Fazendo uma mudança de variável na integral (6.7) para $\lambda=\frac{k^{2}}{2}$ obtemos

$$
\Phi_{t}=\Phi_{1, t}+\Phi_{2, t}
$$

onde

$$
\Phi_{j, t}(x, y):=\int_{0}^{\infty} \frac{1}{\sqrt{2 \lambda}} \chi_{j}(\lambda) e^{-i \lambda t} \operatorname{Re}\left(\Psi_{+}(x, \sqrt{2 \lambda}) \overline{\Psi_{+}(y, \sqrt{2 \lambda})}\right) d \lambda, j=1,2,
$$

e $\Psi_{+}(x,-k)=\overline{\Psi_{+}(x, k)}$. Denotemos,

$$
h_{j}(\lambda, x, y)=\frac{1}{\sqrt{2 \lambda}} \chi_{j}(\lambda) \operatorname{Re}\left(\Psi_{+}(x, \sqrt{2 \lambda}) \overline{\Psi_{+}(y, \sqrt{2 \lambda})}\right), j=1,2
$$

Das relações

$$
\Psi_{+}(x, \sqrt{2 \lambda}) \overline{\Psi_{+}(y, \sqrt{2 \lambda})}=\frac{1}{2 \pi} \begin{cases}\frac{2 \lambda}{2 \lambda+q^{2}} e^{i \sqrt{2 \lambda}(x-y)}, & x \geq 0 \text { e } y \geq 0 ; \\ \frac{(2 \lambda-q \sqrt{2 \lambda} i) e^{i \sqrt{2 \lambda}(x-y)}+2 \lambda e^{i \sqrt{2 \lambda}(x+y)}}{2 \lambda+q^{2}}, & x \geq 0 \text { e } y \leq 0 ; \\ e^{i \sqrt{2 \lambda}(x-y)}+\frac{q(\sqrt{2 \lambda} i-q)}{2 \lambda+q^{2}} e^{i \sqrt{2 \lambda}(x+y)} & x \leq 0 \text { e } y \geq 0 ; \\ +\frac{q(-\sqrt{2 \lambda} i-q)}{2 \lambda+q^{2}} e^{i \sqrt{2 \lambda}(-(x+y))}+\frac{q^{2}}{2 \lambda+q^{2}} e^{i \sqrt{2 \lambda}(-x+y)} & ,\end{cases}
$$


temos, para $j=1,2$,

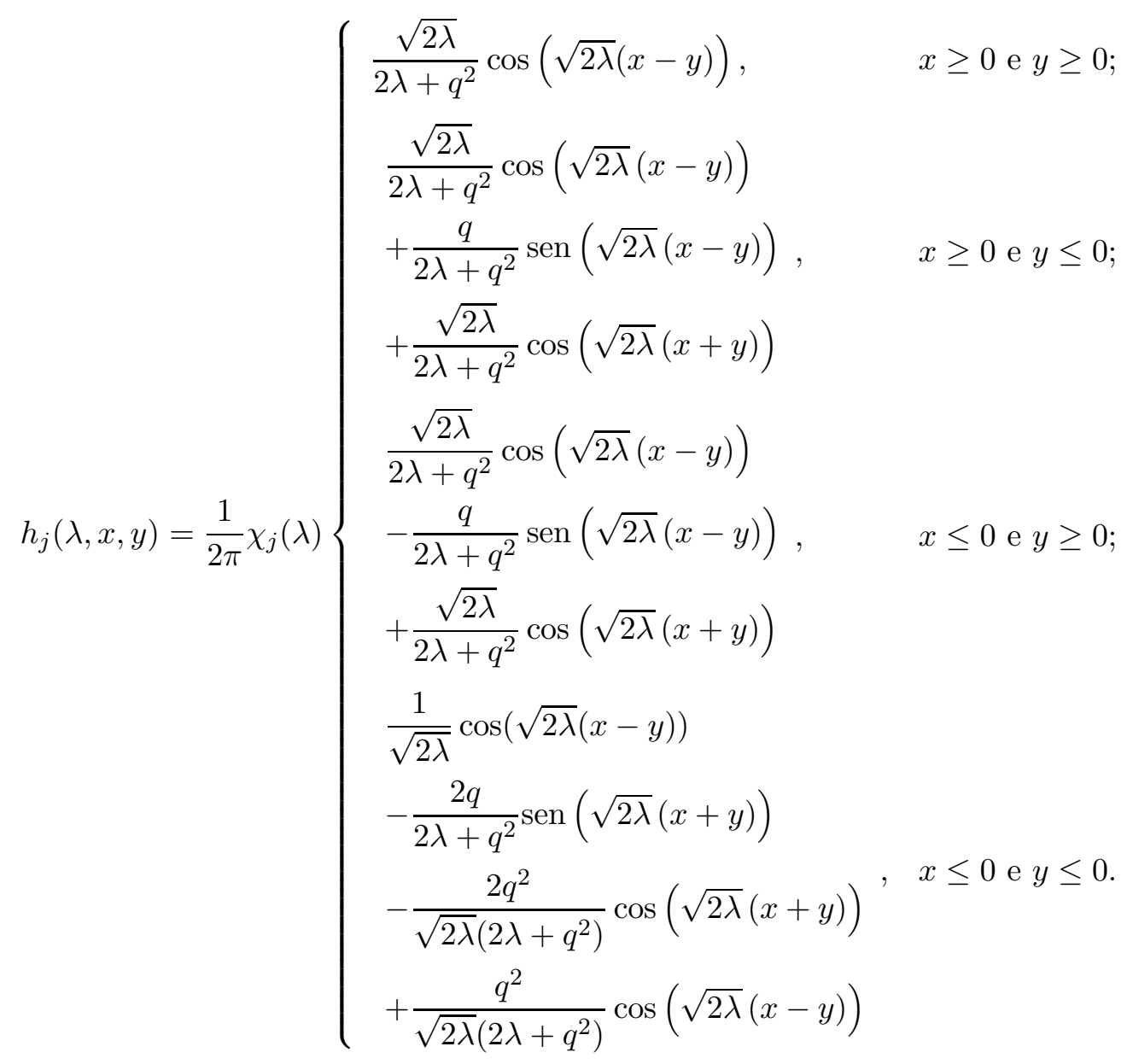

Como nossa $h_{j}$ está definida por partes, dividiremos nosso estudo em quatro casos. Ao longo dessa demonstração será necessário obter $\frac{d h_{j}}{d \lambda}(\lambda, x, y)$ e $\frac{d^{2} h_{j}}{d \lambda^{2}}(\lambda, x, y)$, sendo assim tais derivadas poderão ser consultadas no Apêndice B.1.

$1^{\mathbf{o}}$ Caso : $x \geq 0$ e $y \geq 0$ : Inicialmente estimamos $\Phi_{1, t}(x, y)$. Note que

$$
\left|h_{1}(\lambda, x, y)\right| \leq C \frac{\sqrt{\lambda}}{1+\sqrt{\lambda}} \quad \text { e } \quad\left|\frac{d h_{1}}{d \lambda}(\lambda, x, y)\right| \leq C \frac{1}{\sqrt{\lambda}}(1+|x|)(1+|y|)
$$

Agora estendemos $h_{1}$ para uma função definida para $\lambda \in \mathbb{R}$ definindo $h_{1}(\lambda, x, y)=0$ para $\lambda \leq 0$. Consideramos $t>0$. Como $e^{i \pi}=-1$, teremos imediatamente

$$
\begin{aligned}
\Phi_{1, t}(x, y)= & \frac{1}{2}\left[\int_{0}^{\infty} e^{-i t \lambda} h_{1}(\lambda, x, y) d \lambda-\int_{0}^{\infty} e^{-i t\left(\lambda-\frac{\pi}{t}\right)} h_{1}(\lambda, x, y) d \lambda\right] \\
= & \frac{1}{2}\left[\int_{0}^{\infty} e^{-i t \lambda} h_{1}(\lambda, x, y) d \lambda-\int_{-\frac{\pi}{t}}^{\infty} e^{-i t \lambda} h_{1}\left(\lambda+\frac{\pi}{t}, x, y\right) d \lambda\right] \\
= & \frac{1}{2}\left[\int_{0}^{\infty} e^{-i t \lambda}\left(h_{1}(\lambda, x, y)-h_{1}\left(\lambda+\frac{\pi}{t}, x, y\right)\right) d \lambda\right] \\
& -\frac{1}{2}\left[\int_{-\frac{\pi}{t}}^{0} e^{-i t \lambda} h_{1}\left(\lambda+\frac{\pi}{t}, x, y\right) d \lambda\right] .
\end{aligned}
$$


Logo,

$$
\begin{aligned}
\left|\Phi_{1, t}(x, y)\right| & \leq C \int_{0}^{\infty}\left|h_{1}(\lambda, x, y)-h_{1}\left(\lambda+\frac{\pi}{t}, x, y\right)\right| d \lambda+C \int_{0}^{\frac{\pi}{t}}\left|h_{1}(\lambda, x, y)\right| d \lambda \\
& \leq 2 C \int_{0}^{\frac{2 \pi}{t}}\left|h_{1}(\lambda, x, y)\right| d \lambda+C \int_{\frac{\pi}{t}}^{\infty} \int_{\frac{\pi}{t}}^{\infty}\left|h_{1}(\lambda, x, y)-h_{1}\left(\lambda+\frac{\pi}{t}, x, y\right)\right| d \lambda .
\end{aligned}
$$

Se $t<0$, a mudança de variável que faremos é $\lambda^{\prime}=\lambda+\frac{\pi}{t}$. Inicialmente temos que

$$
\begin{aligned}
\int_{0}^{\frac{2 \pi}{t}}\left|h_{1}(\lambda, x, y)\right| d \lambda & \leq C\left(\frac{2 \pi}{t}-2 \sqrt{\frac{2 \pi}{t}}+2 \ln \left(1+\sqrt{\frac{2 \pi}{t}}\right)\right) \\
& =\frac{C}{t^{3 / 2}}\left(2 \pi \sqrt{t}-2 \sqrt{2 \pi} t+2 t^{3 / 2} \ln \left(1+\sqrt{\frac{2 \pi}{t}}\right)\right) \leq \frac{C_{1}}{t^{3 / 2}}(1+|x|)(1+|y|),
\end{aligned}
$$

onde $C_{1}=\frac{4 \sqrt{2} C}{3} \pi^{\frac{3}{2}}$ (veja Apêndice B.2). Agora estimaremos a segunda parcela da desigualdade acima. Para isso, dividiremos em dois casos:

I) $\frac{\pi}{t} \geq 1$ : Então $\frac{2 \pi}{t} \geq 2$ e assim $\chi_{1}(\lambda)=0$ para $\lambda \geq \frac{2 \pi}{t} \geq 2$. Então,

$$
\begin{aligned}
& \int_{\frac{\pi}{t}}^{\infty}\left|h_{1}(\lambda, x, y)-h_{1}\left(\lambda+\frac{\pi}{t}, x, y\right)\right| d \lambda=\int_{\frac{\pi}{t}}^{\frac{2 \pi}{t}}\left|h_{1}(\lambda, x, y)-h_{1}\left(\lambda+\frac{\pi}{t}, x, y\right)\right| d \lambda \\
= & \int_{\frac{\pi}{t}}^{\frac{2 \pi}{t}} \int_{\lambda}^{\lambda+\frac{\pi}{t}}\left|\frac{d h_{1}}{d \rho}(\rho, x, y)\right| d \rho d \lambda \leq C \int_{\frac{\pi}{t}}^{\frac{2 \pi}{t}} \int_{\lambda}^{\lambda+\frac{\pi}{t}} \frac{1}{\sqrt{\rho}} d \rho d \lambda(1+|x|)(1+|y|) \\
\leq & C \frac{\pi}{t} \int_{\frac{\pi}{t}}^{\frac{2 \pi}{t}} \frac{1}{\sqrt{\lambda}} d \lambda(1+|x|)(1+|y|) .
\end{aligned}
$$

Observe que $\frac{\pi}{t}<\lambda$, então $\frac{1}{\sqrt{\lambda}}<\frac{\sqrt{t}}{\sqrt{\pi}}$, $\log 0$

$$
\begin{aligned}
\int_{\frac{\pi}{t}}^{\infty}\left|h_{1}(\lambda, x, y)-h_{1}\left(\lambda+\frac{\pi}{t}, x, y\right)\right| d \lambda & \leq C \frac{\pi}{t} \frac{\sqrt{t}}{\sqrt{\pi}} \int_{\frac{\pi}{t}}^{\frac{2 \pi}{t}} d \lambda(1+|x|)(1+|y|) \\
& \leq C \frac{\pi^{\frac{3}{2}}}{t^{\frac{3}{2}}}(1+|x|)(1+|y|) .
\end{aligned}
$$

II) $\frac{\pi}{t} \leq 1$ : Aqui precisamos analisar dois sub-casos: $\frac{1}{2} \leq \frac{\pi}{t}$ e $\frac{1}{2} \geq \frac{\pi}{t}$.

i) $\frac{1}{2} \leq \frac{\pi}{t}$ : Nesse caso $1-\frac{\pi}{t} \leq \frac{\pi}{t}$ e como $\frac{\pi}{t} \leq 1$, teremos $2-\frac{\pi}{t} \geq 1$. Vejamos uma ilustração na Figura 6.1.

Então temos

$$
\begin{aligned}
& \int_{\frac{\pi}{t}}^{\infty}\left|h_{1}(\lambda, x, y)-h_{1}\left(\lambda+\frac{\pi}{t}, x, y\right)\right| d \lambda=\int_{\frac{\pi}{t}}^{2}\left|h_{1}(\lambda, x, y)-h_{1}\left(\lambda+\frac{\pi}{t}, x, y\right)\right| d \lambda \\
= & \int_{\frac{\pi}{t}}^{1}\left|h_{1}(\lambda, x, y)-h_{1}\left(\lambda+\frac{\pi}{t}, x, y\right)\right| d \lambda+\int_{1}^{2-\frac{\pi}{t}}\left|h_{1}(\lambda, x, y)-h_{1}\left(\lambda+\frac{\pi}{t}, x, y\right)\right| d \lambda \\
& +\int_{2-\frac{\pi}{t}}^{2}\left|h_{1}(\lambda, x, y)-h_{1}\left(\lambda+\frac{\pi}{t}, x, y\right)\right| d \lambda .
\end{aligned}
$$




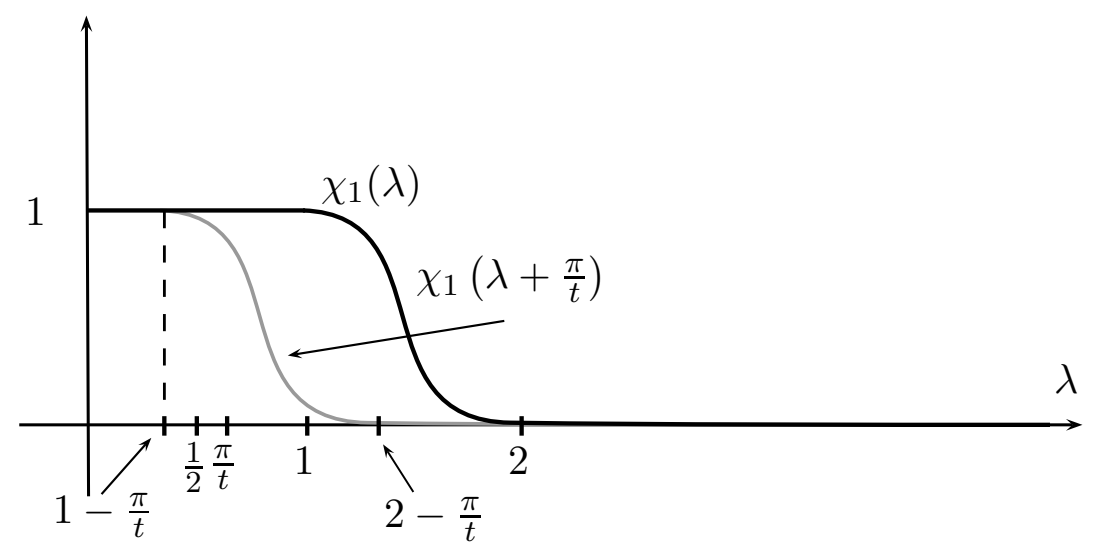

Figura 6.1: $\chi_{1}(\lambda)$ e $\chi_{1}\left(\lambda+\frac{\pi}{t}\right)$

Como $1-\frac{\pi}{t} \leq \frac{\pi}{t}$

$$
\begin{aligned}
& \int_{\frac{\pi}{t}}^{1}\left|h_{1}(\lambda, x, y)-h_{1}\left(\lambda+\frac{\pi}{t}, x, y\right)\right| d \lambda \leq \int_{\frac{\pi}{t}}^{1} \int_{\lambda}^{\lambda+\frac{\pi}{t}}\left|\frac{d h_{1}}{d \rho}(\rho, x, y)\right| d \rho d \lambda \\
& \leq C \int_{\frac{\pi}{t}}^{1} \int_{\lambda}^{\lambda+\frac{\pi}{t}} \frac{1}{\sqrt{\rho}} d \rho d \lambda(1+|x|)(1+|y|) \leq C \frac{\sqrt{t}}{\sqrt{\pi}} \frac{\pi}{t}\left(1+\frac{\pi}{t}\right) \\
& \leq C_{1} \frac{\pi^{\frac{3}{2}}}{t^{\frac{3}{2}}}(1+|x|)(1+|y|),
\end{aligned}
$$

e

$$
\begin{aligned}
& \int_{1}^{2-\frac{\pi}{t}}\left|h_{1}(\lambda, x, y)-h_{1}\left(\lambda+\frac{\pi}{t}, x, y\right)\right| d \lambda \leq \int_{1}^{2-\frac{\pi}{t}} \int_{\lambda}^{\lambda+\frac{\pi}{t}}\left|\frac{d h_{1}}{d \rho}(\rho, x, y)\right| d \rho d \lambda \\
& \leq C \int_{1}^{2-\frac{\pi}{t}} \int_{\lambda}^{\lambda+\frac{\pi}{t}} \frac{1}{\sqrt{\rho}} d \rho d \lambda(1+|x|)(1+|y|) \\
& \stackrel{1<\lambda<\rho}{\leq} \quad C \int_{1}^{2-\frac{\pi}{t}} \int_{\lambda}^{\lambda+\frac{\pi}{t}} 1 d \rho d \lambda(1+|x|)(1+|y|) \\
& \leq \quad C \int_{1}^{2-\frac{\pi}{t}} \frac{\pi}{t} d \lambda(1+|x|)(1+|y|) \leq C \frac{\pi}{t}\left(1-\frac{\pi}{t}\right)(1+|x|)(1+|y|) \\
& \leq \quad C \frac{\pi^{2}}{t^{2}}(1+|x|)(1+|y|) \stackrel{\pi \leq t}{\leq} C \frac{\pi^{\frac{3}{2}}}{t^{\frac{3}{2}}}(1+|x|)(1+|y|)
\end{aligned}
$$

e como $2-\frac{\pi}{t} \geq 1$,

$$
\begin{aligned}
& \int_{2-\frac{\pi}{t}}^{2}\left|h_{1}(\lambda, x, y)-h_{1}\left(\lambda+\frac{\pi}{t}, x, y\right)\right| d \lambda \leq \int_{2-\frac{\pi}{t}}^{2} \int_{\lambda}^{\lambda+\frac{\pi}{t}}\left|\frac{d h_{1}}{d \rho}(\rho, x, y)\right| d \rho d \lambda \\
\leq & C \int_{2-\frac{\pi}{t}}^{2} \int_{\lambda}^{\lambda+\frac{\pi}{t}} \frac{1}{\sqrt{\rho}} d \rho d \lambda(1+|x|)(1+|y|)
\end{aligned}
$$




$$
\begin{aligned}
& \stackrel{2-\frac{\pi}{t}<\lambda<\rho}{\leq} C \frac{\pi}{t} \int_{1}^{2-\frac{\pi}{t}} \frac{1}{\sqrt{2-\frac{\pi}{t}}} d \lambda(1+|x|)(1+|y|) \leq C \frac{\pi^{2}}{t^{2}} \frac{1}{\sqrt{2-\frac{\pi}{t}}}(1+|x|)(1+|y|) \\
& \quad \leq \quad C \frac{\pi^{2}}{t^{2}}(1+|x|)(1+|y|) \stackrel{\pi \leq t}{\leq} C \frac{\pi^{\frac{3}{2}}}{t^{\frac{3}{2}}}(1+|x|)(1+|y|) .
\end{aligned}
$$

Assim, nesse caso onde $\pi \leq t \leq 2 \pi$, temos

$$
\int_{\frac{\pi}{t}}^{\infty}\left|h_{1}(\lambda, x, y)-h_{1}\left(\lambda+\frac{\pi}{t}, x, y\right)\right| d \lambda \leq \frac{C}{t^{\frac{3}{2}}}(1+|x|)(1+|y|) .
$$

ii) $\frac{\pi}{t} \leq \frac{1}{2}$ : Iniciamos considerando $2 \pi \leq t \leq 3 \pi$, dessa forma, $t=2 \pi+\tau$, onde $0 \leq \tau \leq$ $\pi$. Então $1 \leq \frac{\pi}{\tau}$. Logo,

$$
\Phi_{1, t}(x, y)=\int_{0}^{\infty} e^{-i t \lambda} h_{1}(\lambda, x, y) d \lambda=\int_{0}^{\infty} e^{-i \lambda \tau} e^{-2 \pi i \lambda} h_{1}(\lambda, x, y) d \lambda .
$$

Chamando $f(\lambda, x, y)=e^{-2 \pi i \lambda} h_{1}(\lambda, x, y)$, temos

$$
\begin{aligned}
\left|\Phi_{1, t}(x, y)\right| & \leq C \int_{0}^{\infty}\left|f(\lambda, x, y)-f\left(\lambda+\frac{\pi}{\tau}, x, y\right)\right| d \lambda+C \int_{0}^{\frac{\pi}{\tau}}|f(\lambda, x, y)| d \lambda \\
& \leq 2 C \int_{0}^{\frac{2 \pi}{\tau}}|f(\lambda, x, y)| d \lambda+C \int_{\frac{\pi}{\tau}}^{\infty}\left|f(\lambda, x, y)-f\left(\lambda+\frac{\pi}{\tau}, x, y\right)\right| d \lambda \\
& =2 C \int_{0}^{\frac{2 \pi}{\tau}}\left|h_{1}(\lambda, x, y)\right| d \lambda+C \int_{\frac{\pi}{\tau}}^{\infty}\left|f(\lambda, x, y)-f\left(\lambda+\frac{\pi}{\tau}, x, y\right)\right| d \lambda .
\end{aligned}
$$

Agora

$$
\begin{aligned}
& \int_{\frac{\pi}{\tau}}^{\infty}\left|f(\lambda, x, y)-f\left(\lambda+\frac{\pi}{\tau}, x, y\right)\right| d \lambda \\
= & \int_{\frac{\pi}{\tau}}^{\infty}\left|e^{-2 \pi i \lambda} h_{1}(\lambda, x, y)-e^{-2 \pi i \lambda} e^{-i \frac{2 \pi^{2}}{\tau}} h_{1}\left(\lambda+\frac{\pi}{\tau}, x, y\right)\right| d \lambda \\
= & \int_{\frac{\pi}{\tau}}^{\infty}\left|h_{1}(\lambda, x, y)-e^{-i \frac{2 \pi^{2}}{\tau}} h_{1}\left(\lambda+\frac{\pi}{\tau}, x, y\right)\right| d \lambda \\
\leq & \int_{\frac{\pi}{\tau}}^{\infty}\left|h_{1}(\lambda, x, y)-h_{1}\left(\lambda+\frac{\pi}{\tau}, x, y\right)\right| d \lambda+\int_{\frac{\pi}{\tau}}^{\infty}\left|\left(1-e^{-i \frac{2 \pi^{2}}{\tau}}\right) h_{1}\left(\lambda+\frac{\pi}{\tau}, x, y\right)\right| d \lambda,
\end{aligned}
$$

mas, como $\frac{2 \pi}{\tau} \geq 2$,

$$
\begin{aligned}
\int_{\frac{\pi}{\tau}}^{\infty}\left|\left(1-e^{-i \frac{2 \pi^{2}}{\tau}}\right) h_{1}\left(\lambda+\frac{\pi}{\tau}, x, y\right)\right| d \lambda & \leq 2 \int_{\frac{\pi}{\tau}}^{\infty}\left|h_{1}\left(\lambda+\frac{\pi}{\tau}, x, y\right)\right| d \lambda \\
& =2 \int_{\frac{2 \pi}{\tau}}^{\infty}\left|h_{1}(\lambda, x, y)\right| d \lambda=0 .
\end{aligned}
$$

Logo,

$\left|\Phi_{1, t}(x, y)\right| \leq 2 C \int_{0}^{\frac{2 \pi}{\tau}}\left|h_{1}(\lambda, x, y)\right| d \lambda+C \int_{\frac{\pi}{\tau}}^{\infty}\left|h_{1}(\lambda, x, y)-h_{1}\left(\lambda+\frac{\pi}{\tau}, x, y\right)\right| d \lambda$, 
e como $1 \leq \frac{\pi}{\tau}$, obtemos imediatamente da análise acima que

$$
\left|\Phi_{1, t}(x, y)\right| \leq \frac{C}{t^{\frac{3}{2}}}(1+|x|)(1+|y|) .
$$

De maneira similar mostramos uma desigualdade como essa de (6.15) para $\Phi_{1, t}$, para $n \pi \leq t \leq(n+1) \pi$, com $n \geq 3$ natural.

Agora estimaremos $\Phi_{2, t}(x, y)$. Temos

$$
\Phi_{2, t}(x, y)=\int_{0}^{\infty} e^{-i \lambda t} h_{2}(\lambda, x, y) d \lambda
$$

Integrando por partes (chamando $u=h_{2}(\lambda, x, y)$ e $d v=e^{-i \lambda t} d \lambda$ ), temos

$$
\int e^{-i \lambda t} h_{2}(\lambda, x, y) d \lambda=\frac{i}{t} e^{-i \lambda t} h_{2}(\lambda, x, y)-\int \frac{i}{t} e^{-i \lambda t} \frac{d h_{2}}{d \lambda}(\lambda, x, y) d \lambda .
$$

Integrando mais uma vez por partes (chamando $u=\frac{d h_{2}}{d \lambda}(\lambda, x, y)$ e $d v=\frac{i}{t} e^{-i \lambda t} d \lambda$ ),

$$
\int \frac{i}{t} e^{-i \lambda t} \frac{d}{d \lambda} h_{2}(\lambda, x, y) d \lambda=-\frac{1}{t^{2}} e^{-i \lambda t} \frac{d h_{2}}{d \lambda}(\lambda, x, y)+\int \frac{1}{t^{2}} e^{-i \lambda t} \frac{d^{2} h_{2}}{d \lambda^{2}}(\lambda, x, y) d \lambda,
$$

ou seja,

$$
\begin{aligned}
\int_{0}^{\infty} e^{-i \lambda t} h_{2}(\lambda, x, y) d \lambda= & \left.\frac{i}{t} e^{-i \lambda t} h_{2}(\lambda, x, y)\right|_{0} ^{\infty}+\left.\frac{1}{t^{2}} e^{-i \lambda t} \frac{d h_{2}}{d \lambda}(\lambda, x, y)\right|_{0} ^{\infty} \\
& -\frac{1}{t^{2}} \int_{0}^{\infty} e^{-i \lambda t} \frac{d^{2} h_{2}}{d \lambda^{2}}(\lambda, x, y) d \lambda
\end{aligned}
$$

Todavia, $\lim _{\lambda \rightarrow 0^{+}} h_{2}(\lambda, x, y)=\lim _{\lambda \rightarrow 0^{+}} \frac{d h_{2}}{d \lambda}(\lambda, x, y)=\lim _{\lambda \rightarrow \infty}\left|h_{2}(\lambda, x, y)\right|=\lim _{\lambda \rightarrow \infty}\left|\frac{d h_{2}}{d \lambda}(\lambda, x, y)\right|=0$, então

$$
\left|\int_{0}^{\infty} e^{-i \lambda t} h_{2}(\lambda, x, y) d \lambda\right| \leq \frac{1}{t^{2}} \int_{0}^{\infty}\left|\frac{d^{2} h_{2}}{d \lambda^{2}}(\lambda, x, y)\right| d \lambda
$$

Agora

$$
\begin{aligned}
& 2 \pi\left|\frac{d^{2} h_{2}}{d \lambda^{2}}(\lambda, x, y)\right| \leq\left|\chi_{2}^{\prime \prime}(\lambda)\right| \frac{\sqrt{2 \lambda}}{2 \lambda+q^{2}}+2\left|\chi_{2}^{\prime}(\lambda)\right| \frac{1}{\sqrt{2 \lambda}\left(2 \lambda+q^{2}\right)} \\
& +4\left|\chi_{2}^{\prime}(\lambda)\right| \frac{\sqrt{2 \lambda}}{\left(2 \lambda+q^{2}\right)^{2}}+2\left|\chi_{2}^{\prime}(\lambda)\right| \frac{1}{2 \lambda+q^{2}}|x-y|+\left|\chi_{2}(\lambda)\right| \frac{1}{2 \sqrt{2}\left(2 \lambda+q^{2}\right) \lambda^{3 / 2}} \\
& +4\left|\chi_{2}(\lambda)\right| \frac{1}{\sqrt{2 \lambda}\left(2 \lambda+q^{2}\right)^{2}}+\left|\chi_{2}(\lambda)\right| \frac{1}{2 \lambda\left(2 \lambda+q^{2}\right)}|x-y|+8\left|\chi_{2}(\lambda)\right| \frac{\sqrt{2 \lambda}}{\left(2 \lambda+q^{2}\right)^{3}} \\
& +4\left|\chi_{2}(\lambda)\right| \frac{1}{\left(2 \lambda+q^{2}\right)^{2}}|x-y|+\left|\chi_{2}(\lambda)\right| \frac{1}{\sqrt{2 \lambda}\left(2 \lambda+q^{2}\right)}|x-y|^{2} .
\end{aligned}
$$

Lembramos que $\chi_{1}(\lambda)=1$, se $\lambda \leq 1$ e $\chi_{1}(\lambda)=0$, se $\lambda \geq 2$. Logo $\chi_{2}(\lambda)=0$, se $\lambda \leq 1$, e 
$\chi_{2}(\lambda)=1$, se $\lambda \geq 2$. Então, olhando para a integral do lado direito de (6.16) vemos que todas são finitas (veja Seção B.3), então

$$
\int_{0}^{\infty}\left|\frac{d^{2} h_{2}}{d \lambda^{2}}(\lambda, x, y)\right| d \lambda \leq C(1+|x|)^{2}(1+|y|)^{2} .
$$

Então, de (6.16),

$$
\left|\Phi_{2, t}(x, y)\right| \leq \frac{C}{t^{2}}(1+|x|)^{2}(1+|y|)^{2} .
$$

Claramente que para $t \geq 1$, temos $\frac{1}{t^{2}} \leq \frac{1}{t^{3 / 2}}$, ou seja, para $t \geq 1$,

$$
\left|\Phi_{2, t}(x, y)\right| \leq \frac{C}{t^{3 / 2}}(1+|x|)^{2}(1+|y|)^{2},
$$

consequentemente, para $t \geq 1$,

$$
\left|\Phi_{t}(x, y)\right| \leq \frac{C}{t^{3 / 2}}(1+|x|)^{2}(1+|y|)^{2},
$$

mas, de (6.9), $\left|\Phi_{t}(x, y)\right| \leq C$, para todo $t \in \mathbb{R}$. Assim, para todo $t$,

$$
\left|\Phi_{t}(x, y)\right| \leq \frac{C}{(1+|t|)^{\frac{3}{2}}}(1+|x|)^{2}(1+|y|)^{2} .
$$

Portanto, de (6.15) e (6.17), para qualquer $s>1$ e $0 \leq \beta \leq 1$,

$$
(1+|x|)^{-s-2 \beta}\left|\Phi_{t}(x, y)\right|(1+|y|)^{-s-2 \beta} \leq \frac{C}{(1+|t|)^{\frac{1}{2}+\beta}} .
$$

Para obtermos (6.18), basta ver o seguinte: Para $s>1, f_{s}(\beta)=\alpha^{-s-2 \beta} \tau^{\frac{1}{2}+\beta}\left|\Phi_{t}(x, y)\right|$ é convexa $(\alpha>1, \tau>1)$, pois $f_{s}^{\prime \prime}(\beta) \geq 0$, logo, para $\beta \in[0,1], f_{s}(\beta) \leq(1-\beta) f_{s}(0)+\beta f_{s}(1)$. Escolha, $\alpha=(1+|x|)(1+|y|)$ e $\tau=(1+|t|)$. Assim, $f_{s}(0)=[(1+|x|)(1+|y|)]^{-s}(1+$ $|t|)^{\frac{1}{2}}\left|\Phi_{t}(x, y)\right|$ e $f_{s}(1)=[(1+|x|)(1+|y|)]^{-s-2}(1+|t|)^{\frac{3}{2}}\left|\Phi_{t}(x, y)\right|$, então, por (6.10) e (6.17), respectivamente, $f_{s}(0) \leq C$ e $f_{s}(1) \leq C$. Portanto, $f_{s}(\beta) \leq C$.

Finalmente, com as estimativas acima temos:

$$
\begin{aligned}
& \left\|e^{-i t H_{q}} P_{c} f\right\|_{L_{-s-2 \beta}^{2}}^{2} \\
= & \int_{\mathbb{R}}\left|\int_{\mathbb{R}}(1+|x|)^{\frac{-s-2 \beta}{2}} \Phi_{t}(x, y) f(y) d y\right|^{2} d x \\
= & \int_{\mathbb{R}}\left|\int_{\mathbb{R}}(1+|x|)^{\frac{-s-2 \beta}{2}} \Phi_{t}(x, y)(1+|y|)^{-s-2 \beta} f(y)(1+|y|)^{s+2 \beta} d y\right|^{2} d x \\
= & \int_{\mathbb{R}}(1+|x|)^{s+2 \beta}\left|\int_{\mathbb{R}}(1+|x|)^{-s-2 \beta} \Phi_{t}(x, y)(1+|y|)^{-s-2 \beta} f(y)(1+|y|)^{s+2 \beta} d y\right|^{2} d x .
\end{aligned}
$$

Definindo $K(x, y)=(1+|x|)^{-s-2 \beta} \Phi_{t}(x, y)(1+|y|)^{-s-2 \beta}, d \mu(x)=(1+|x|)^{s+2 \beta} d x$ e $d \nu(y)=$ 
$(1+|y|)^{s+2 \beta} d y$, temos

$$
\left\|e^{-i t H_{q}} P_{c} f\right\|_{L_{-s-2 \beta}^{2}}^{2}=\int_{\mathbb{R}}\left|\int_{\mathbb{R}} K(x, y) f(y) d \nu(y)\right|^{2} d \mu(x)
$$

Logo, pelo Lema de Schur (Lema B.4.1),

$$
\left\|e^{-i t H_{q}} P_{c} f\right\|_{L_{-s-2 \beta}^{2}}^{2} \leq c\|f\|_{L_{s+2 \beta}^{2}}^{2}
$$

De fato, por (6.18), temos para todo $x \in \mathbb{R}$

$$
\begin{aligned}
\int_{\mathbb{R}}(1+|x|)^{-s-2 \beta}\left|\Phi_{t}(x, y)\right|(1+|y|)^{-s-2 \beta} d \mu(y) & =\int_{\mathbb{R}}(1+|x|)^{-s-2 \beta}\left|\Phi_{t}(x, y)\right| d y \\
& \leq C \int_{\mathbb{R}} \frac{1}{(1+|t|)^{\frac{1}{2}+\beta}} \frac{1}{(1+|y|)^{s+2 \beta}} d y \\
& \leq \frac{C}{(1+|t|)^{\frac{1}{2}+\beta}}=A
\end{aligned}
$$

e para todo $y \in \mathbb{R}$

$$
\begin{aligned}
\int_{\mathbb{R}}(1+|x|)^{-s-2 \beta}\left|\Phi_{t}(x, y)\right|(1+|y|)^{-s-2 \beta} d \mu(x) & =\int_{\mathbb{R}}(1+|y|)^{-s-2 \beta}\left|\Phi_{t}(x, y)\right| d x \\
& \leq C \int_{\mathbb{R}} \frac{1}{(1+|t|)^{\frac{1}{2}+\beta}} \frac{1}{(1+|x|)^{s+2 \beta}} d x \\
& \leq \frac{C}{(1+|t|)^{\frac{1}{2}+\beta}}=B
\end{aligned}
$$

então, pelo Lema de Schur, $c=A^{1-\frac{1}{2}} B^{\frac{1}{2}}=\frac{C}{(1+|t|)^{\frac{1}{2}+\beta}}$ e obtemos (6.19). Isto finaliza o caso $x \geq 0$ e $y \geq 0$, com

$$
\left\|e^{-i t H_{q}} P_{c} f\right\|_{L_{-s-2 \beta}^{2}}^{2} \leq \frac{C}{(1+|t|)^{\frac{1}{2}+\beta}}\|f\|_{L_{s+2 \beta}^{2}}^{2}
$$

para todo $t \in \mathbb{R}$.

$2^{\mathbf{o}}$ Caso : $x \geq 0$ e $y \leq 0:$ Note que $\operatorname{sen}(\sqrt{2 \lambda}(x-y)) \leq C \sqrt{2 \lambda}(1+|x|)(1+|y|)$, então

$$
\left|h_{1}(\lambda, x, y)\right| \leq C \frac{\sqrt{\lambda}}{1+\sqrt{\lambda}}(1+|x|)(1+|y|) \quad \text { e } \quad\left|\frac{d h_{1}}{d \lambda}(\lambda, x, y)\right| \leq C \frac{1}{\sqrt{\lambda}}(1+|x|)^{2}(1+|y|)^{2} .
$$

Também temos $\lim _{\lambda \rightarrow 0^{+}} h_{2}(\lambda, x, y)=\lim _{\lambda \rightarrow 0^{+}} \frac{d h_{2}}{d \lambda}(\lambda, x, y)=\lim _{\lambda \rightarrow \infty}\left|h_{2}(\lambda, x, y)\right|=\lim _{\lambda \rightarrow \infty}\left|\frac{d h_{2}}{d \lambda}(\lambda, x, y)\right|=$ 0 e as parcelas da integral $\int_{0}^{\infty}\left|\frac{d^{2} h_{2}}{d \lambda^{2}}(\lambda, x, y)\right| d \lambda$ são todas finitas. Portanto, segue como o $1^{\circ}$ Caso.

$3^{\mathbf{o}}$ Caso : $x \leq 0$ e $y \geq 0$ : A menos de um sinal, segue como o $\mathbf{2}^{\mathbf{o}}$ Caso. 
$4^{\mathbf{o}}$ Caso : $x \leq 0$ e $y \leq 0$ : Podemos escrever $h_{1}$ da seguinte forma:

$$
\begin{aligned}
2 \pi h_{1}(\lambda, x, y)= & \chi_{1}(\lambda) \frac{2 \lambda \cos (\sqrt{2 \lambda}(x-y))-2 q \sqrt{2 \lambda} \operatorname{sen}(\sqrt{2 \lambda}(x+y))}{\sqrt{2 \lambda}\left(2 \lambda+q^{2}\right)} \\
& +\chi_{1}(\lambda) \frac{2 q^{2}(\cos (\sqrt{2 \lambda}(x-y))-\cos (\sqrt{2 \lambda}(x+y)))}{\sqrt{2 \lambda}\left(2 \lambda+q^{2}\right)} \\
= & \chi_{1}(\lambda) \frac{2 \lambda \cos (\sqrt{2 \lambda}(x-y))-2 q \sqrt{2 \lambda} \operatorname{sen}(\sqrt{2 \lambda}(x+y))}{\sqrt{2 \lambda}\left(2 \lambda+q^{2}\right)} \\
& +\chi_{1}(\lambda) \frac{4 q^{2} \operatorname{sen}(\sqrt{2 \lambda} x) \operatorname{sen}(\sqrt{2 \lambda} y)}{\sqrt{2 \lambda}\left(2 \lambda+q^{2}\right)}
\end{aligned}
$$

Quando $\lambda=0$, teremos em $h_{1}$ uma indeterminação do tipo zero sobre zero. Usando L'Hôpital teremos $\lim _{\lambda \rightarrow 0^{+}} h_{1}(\lambda, x, y)=0$. Como $\operatorname{sen}(\sqrt{2 \lambda} z) \leq C \sqrt{2 \lambda}(1+|z|)$, obtemos

$$
\left|h_{1}(\lambda, x, y)\right| \leq C \frac{\sqrt{\lambda}}{1+\sqrt{\lambda}}(1+|x|)(1+|y|)
$$

Agora vamos obter uma limitação para $\left|\frac{d h_{1}}{d \lambda}(\lambda, x, y)\right|$. Olhando para $\frac{d h_{1}}{d \lambda}(\lambda, x, y)$ no Apêndice B.1, exceto para os termos $J_{1}, J_{2}$ e $J_{3}$ nesse caso, podemos perceber que o restante pode ser limitado por $\frac{1}{\sqrt{\lambda}}(1+|x|)^{2}(1+|y|)^{2}$. Por outro lado, chamando $J=J_{1}+J_{2}+J_{3}$, temos

$$
\begin{aligned}
J & =-\chi_{i}(\lambda) \frac{\cos (\sqrt{2 \lambda}(x-y))}{(2 \lambda)^{3 / 2}}+\chi_{i}(\lambda) \frac{2 q^{2} \cos (\sqrt{2 \lambda}(x+y))}{(2 \lambda)^{3 / 2}\left(2 \lambda+q^{2}\right)}-\chi_{i}(\lambda) \frac{q^{2} \cos (\sqrt{2 \lambda}(x-y))}{(2 \lambda)^{3 / 2}\left(2 \lambda+q^{2}\right)} \\
& =\chi_{i}(\lambda)\left[-\frac{2 \lambda \cos (\sqrt{2 \lambda}(x-y))+2 q^{2}(\cos (\sqrt{2 \lambda}(x+y))-\cos (\sqrt{2 \lambda}(x-y)))}{(2 \lambda)^{3 / 2}\left(2 \lambda+q^{2}\right)}\right] \\
& =\chi_{i}(\lambda)\left[-\frac{2 \lambda \cos (\sqrt{2 \lambda}(x-y))}{(2 \lambda)^{3 / 2}\left(2 \lambda+q^{2}\right)}+\frac{4 q^{2} \operatorname{sen}(\sqrt{2 \lambda} x) \operatorname{sen}(\sqrt{2 \lambda} y)}{(2 \lambda)^{3 / 2}\left(2 \lambda+q^{2}\right)}\right] \\
& \leq \chi_{i}(\lambda)\left[-\frac{1}{\sqrt{2 \lambda}} \frac{\cos (\sqrt{2 \lambda}(x-y))}{2 \lambda+q^{2}}+4 C q^{2} \frac{2 \lambda(1+|x|)(1+|y|)}{(2 \lambda)^{3 / 2}\left(2 \lambda+q^{2}\right)}\right],
\end{aligned}
$$

então

$$
|J| \leq C \frac{1}{\sqrt{\lambda}}(1+|x|)(1+|y|) .
$$

Logo,

$$
\left|\frac{d h_{1}}{d \lambda}(\lambda, x, y)\right| \leq C \frac{1}{\sqrt{\lambda}}(1+|x|)^{2}(1+|y|)^{2} .
$$

Também temos $\lim _{\lambda \rightarrow 0^{+}} h_{2}(\lambda, x, y)=\lim _{\lambda \rightarrow 0^{+}} \frac{d h_{2}}{d \lambda}(\lambda, x, y)=\lim _{\lambda \rightarrow \infty}\left|h_{2}(\lambda, x, y)\right|=\lim _{\lambda \rightarrow \infty}\left|\frac{d h_{2}}{d \lambda}(\lambda, x, y)\right|=$ 0 e as parcelas da integral $\int_{0}^{\infty}\left|\frac{d^{2} h_{2}}{d \lambda^{2}}(\lambda, x, y)\right| d \lambda$ são todas finitas. 
Desta forma, vemos que tal caso segue do $\mathbf{1}^{\mathbf{0}}$ Caso. Isto finaliza a demonstração do Teorema.

\subsection{Aproximação à Variedade Centro}

Nessa seção demonstraremos que soluções começando próximas da variedade centro aproximarão dessa variedade. Recordemos que escrevemos a solução $u(t)$ da equação de Schrödinger (3.1) como $u(t)=u_{p}(t) \psi_{0}+u_{c}(t)$, onde $u_{c}(t) \in \mathscr{R}\left(P_{c}\right)$, enquanto que a variedade centro pode ser escrita como $\psi(t)=u_{p}(t) \psi_{0}+h\left(u_{p}(t)\right)$ em uma vizinhança da origem de $L_{\sigma}^{2} \cap H^{1}$.

Defina

$$
\left\{\begin{aligned}
\varepsilon & =\|u(0)\|_{L_{\sigma}^{2} \cap H^{1}}, \\
z(t) & =u(t)-\psi(t)=u_{c}(t)-h\left(u_{p}(t)\right) \in \mathscr{R}\left(P_{c}\right) .
\end{aligned}\right.
$$

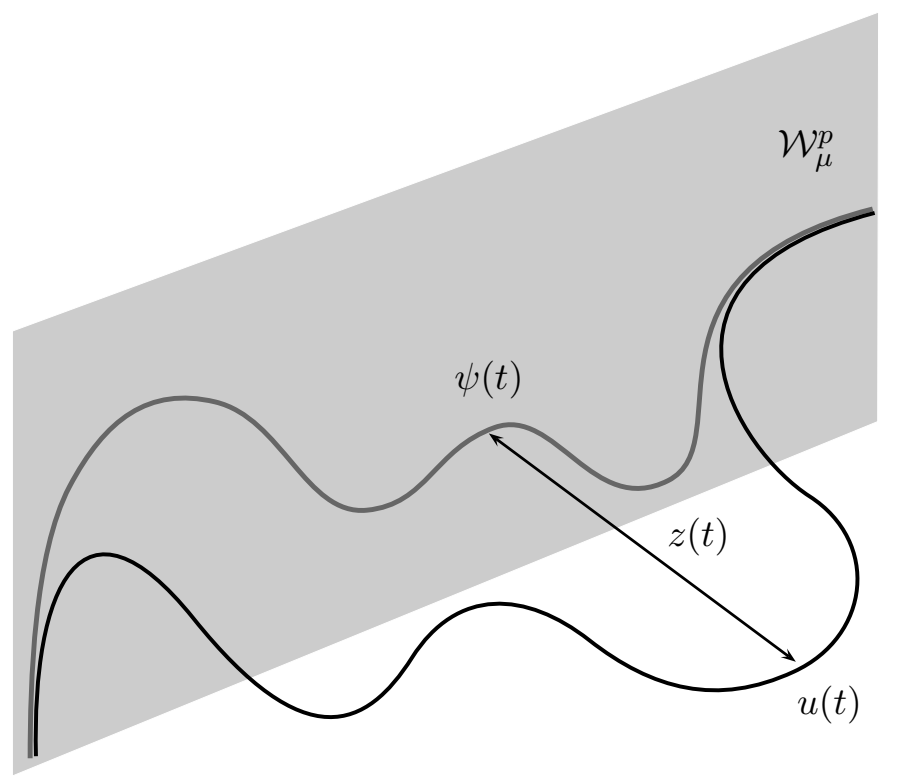

Figura 6.2: Aproximação à variedade centro

Provaremos que para qualquer solução de (3.1) para os quais $\varepsilon$ é suficientemente pequeno e também $u(0) \in H_{2}^{2}(\Omega)$, então

$$
z(t) \rightarrow 0, \text { quando } t \rightarrow \infty
$$

em $L_{-\sigma}^{2}(\mathbb{R})$, e portanto a solução aproxima-se da variedade invariante nessa norma. (Figura 6.2)

Iniciamos com os seguintes lemas:

Lema 6.2.1. Sejam $G(z)=|z|^{p} z, z \in \mathbb{C}, a, b \in \mathbb{C}$ e $p>1$, então

$$
|G(a+b)-G(a)| \leq C\left(|b|^{p}+|a|^{p}\right)|b|
$$

onde $C$ é uma constante real. 
Lema 6.2.2. Se $u_{c}(0)=u(0) \in H_{1}^{1}(\mathbb{R}) \cap H_{2}^{2}(\Omega) \cap \mathscr{R}\left(P_{c}\right)$, então existe $T=T\left(\|u(0)\|_{H_{1}^{1}(\mathbb{R}) \cap H_{2}^{2}(\Omega)}\right)>$ 0 e uma única solução $u_{c}$ em $[0, T]$ do problema (3.1), tal que

$$
\langle x\rangle^{\frac{2 \sigma}{p}} u_{c} \in C\left([0, T] ; H^{1}(\mathbb{R})\right),
$$

desde que $2 \sigma \leq p$.

Demonstração: Se $u_{c}(0) \in H_{1}^{1}(\mathbb{R}) \cap H_{2}^{2}(\Omega) \cap \mathscr{R}\left(P_{c}\right)$, então pelo Teorema 5.3.2, existe $T>0$ e uma única solução $u_{c}$ de $(3.1)$ com $u_{c} \in C\left([0, T] ; H_{1}^{1}(\mathbb{R}) \cap H_{2}^{2}(\Omega) \cap \mathscr{R}\left(P_{c}\right)\right)$.

Para $\phi \in H^{1}(\mathbb{R}) \cap H^{2}(\Omega)$, temos $\|\phi\|_{H^{1}(\mathbb{R}) \cap H^{2}(\Omega)}=\int_{\Omega}|\phi|^{2} d x+\int_{\Omega}\left|\phi^{\prime}\right|^{2} d x+\int_{\Omega}\left|\phi^{\prime \prime}\right|^{2} d x<$ $\infty$; consequentemente, $\|\phi\|_{H^{1}(\mathbb{R})} \leq\|\phi\|_{H^{2}(\Omega)}$. Então, para $t \in[0, T]$, como $\frac{2 \sigma}{p} \leq 1$,

$$
\begin{aligned}
\left\|\langle x\rangle^{\frac{2 \sigma}{p}} u_{c}(t)\right\|_{H^{1}}^{2} & =\left\|\langle x\rangle^{\frac{2 \sigma}{p}} u_{c}(t)\right\|_{2}^{2}+\left\|\frac{d}{d x}\left(\langle x\rangle^{\frac{2 \sigma}{p}} u_{c}(t)\right)\right\|_{2}^{2} \\
& \leq\left\|\langle x\rangle^{\frac{2 \sigma}{p}} u_{c}(t)\right\|_{2}^{2}+\left\|\frac{2 \sigma}{p} \frac{x}{\langle x\rangle^{2-\frac{2 \sigma}{p}}} u_{c}(t)\right\|_{2}^{2}+\left\|\langle x\rangle^{\frac{2 \sigma}{p}} \frac{d u_{c}(t)}{d x}\right\|_{2}^{2} \\
& \leq\left\|\langle x\rangle u_{c}(t)\right\|_{2}^{2}+\left\|x u_{c}(t)\right\|_{2}^{2}+\left\|\langle x\rangle \frac{d u_{c}(t)}{d x}\right\|_{2}^{2} \\
& \leq C\left(\left\|u_{c}(t)\right\|_{2}^{2}+\left\|x u_{c}(t)\right\|_{2}^{2}+\left\|\frac{d u_{c}(t)}{d x}\right\|_{2}^{2}+\left\|x \frac{d u_{c}(t)}{d x}\right\|_{2}^{2}\right) \\
& \leq C\left(\left\|u_{c}(t)\right\|_{H_{1}^{1}(\mathbb{R})}+\left\|x \frac{d u_{c}(t)}{d x}\right\|_{2}^{2}\right)
\end{aligned}
$$

Como $u_{c} \in H_{1}^{1}(\mathbb{R}) \cap H_{2}^{2}(\Omega)$, pela equação (5.6), (a qual também é certa para $W_{m}^{m, p}(\Omega)$ ),

$$
\left\|x \frac{d u_{c}(t)}{d x}\right\|_{2}^{2} \leq c\left\|u_{c}(t)\right\|_{H_{2}^{2}(\Omega)}^{2}
$$

portanto, para $t \in[0, T]$,

$$
\sup _{t \in[0, T]}\left\|\langle x\rangle^{\frac{2 \sigma}{p}} u_{c}(t)\right\|_{H^{1}} \leq C \sup _{t \in[0, T]}\left\|u_{c}(t)\right\|_{H_{1}^{1}(\mathbb{R}) \cap H_{2}^{2}(\Omega)}<\infty .
$$

Teorema 6.2.1. Considere o problema (3.1). Seja $s>1$ e $\frac{1}{2}<\beta \leq 1$. Suponhamos que $\|x u(t)\|_{H^{1}}$ é suficientemente pequena para todo $t \in \mathbb{R}$. Suponha $u(0) \in L_{s+2 \beta}^{2} \cap H_{1}^{1}(\mathbb{R}) \cap H_{2}^{2}(\Omega), 2(s+2 \beta) \leq p$ such that $\|u(0)\|_{L_{s+2 \beta}^{2} \cap H^{1}}$ é suficientemente pequena. Então $z(t)$ definido em (6.20) satisfaz a seguinte desigualdade

$$
\|z(t)\|_{L_{-s-2 \beta}^{2}} \leq C_{s+2 \beta}\langle t\rangle^{-\frac{1}{2}-\beta}\left\|P_{c} u(0)-h\left(\left\langle u(0), \psi_{0}\right\rangle\right)\right\|_{L_{\sigma}^{2} \cap H^{1}}, \quad \text { para }|t| \leq T .
$$

Mais ainda, como $\|u(0)\|_{H^{1}} \ll \varepsilon$, então $z(t)$ está definida para todo $t$ (Teorema 3.1.2 e e Teorema 
3.1.4).

Observação 6.2.1. 1) Notamos que do Theorem 6.2.1, p em (3.1), satisfaz $p>4$. De fato, sempre existem $s>1$ e $\frac{1}{2}<\beta \leq 1$ tais que $p \geq 2(s+2 \beta)$.

2) A condição $\|u(0)\|_{L_{s+2 \beta}^{2} \cap H^{1}} \ll \varepsilon$ implica imediatamente que $\|z(0)\|_{L_{s+2 \beta}^{2} \cap H^{1}} \ll \varepsilon$ pela conservação da $L^{2}$-norma (ver (6.32) abaixo).

3) A hipótese $\|x u(t)\|_{H^{1}} \ll \varepsilon$, para todo $t$, é localmente certa pelos resultados no Capítulo 5. (Ver Corolário 5.3.1.1). Acreditamos que esta é válida para todo $t$.

Demonstração: Chame $\sigma=s+2 \beta$. Pelo sistema (3.39), observe que $z(t) \in \mathscr{R}\left(P_{c}\right)$ satisfaz

$$
i \dot{z}(t)=H_{q} z(t)+\lambda N\left(u_{p}(t), z(t)\right)
$$

onde

$$
\begin{aligned}
N\left(u_{p}, z\right)= & \left(f_{c}\left(u_{p}, h\left(u_{p}\right)\right)-f_{c}\left(u_{p}, h\left(u_{p}\right)-z\right)\right) \\
& -D h\left(u_{p}\right)\left[f_{p}\left(u_{p}, h\left(u_{p}\right)\right)-f_{p}\left(u_{p}, h\left(u_{p}\right)-z\right)\right] .
\end{aligned}
$$

Do Teorema 4.2.1 temos que a derivada $D h\left(u_{p}\right)$,

$$
\operatorname{Dh}\left(u_{p}\right): \mathbb{C} \rightarrow L_{\sigma}^{2} \cap H^{1}
$$

é limitada desde que $\left|u_{p}\right|<\delta$; isto é,

$$
\left\|D h\left(u_{p}\right)\right\|_{\mathcal{L}\left(\mathbb{C}, L_{\sigma}^{2} \cap H^{1}\right)}=\sup _{\substack{v \in \mathbb{C} \\\|v\|=1}}\left\|D h\left(u_{p}\right) v\right\|_{L_{\sigma}^{2} \cap H^{1}} \leq M
$$

desde que $\left|u_{p}\right|<\delta$.

Agora, queremos limitar a solução de (6.21) em $L_{-\sigma}^{2}$. Lembramos inicialmente que,

$$
\begin{aligned}
& f_{p}\left(u_{p}, u_{c}\right)=\left\langle G\left(u_{p} \psi_{0}+u_{c}\right), \psi_{0}\right\rangle, \\
& f_{c}\left(u_{p}, u_{c}\right)=P_{c} G\left(u_{p} \psi_{0}+u_{c}\right),
\end{aligned}
$$

onde $G(z)=|z|^{p} z$. Mais ainda, para $\psi_{0} \in L^{1} \cap L^{\infty}$, as projeções $P_{p}: L^{p_{0}} \rightarrow L^{p_{0}}$ e $P_{c}=I-P_{p}$ : $L^{p_{0}} \rightarrow L^{p_{0}}, p_{0} \geq 1$, são operadores limitados; além disso, $P_{p}: L_{\sigma}^{2} \rightarrow L_{\sigma}^{2}$ e $P_{c}=I-P_{p}: L_{\sigma}^{2} \rightarrow L_{\sigma}^{2}$, $\sigma \in \mathbb{R}$, são também operadores limitados. Logo, do Lema 6.2.1, temos que a primeira parcela da equação (6.22) pode ser estimada como

$$
\begin{aligned}
\left\|f_{c}\left(u_{p}, h\left(u_{p}\right)\right)-f_{c}\left(u_{p}, h\left(u_{p}\right)-z\right)\right\|_{L_{\sigma}^{2}} & \leq C\left\|\langle x\rangle^{\sigma}\left(G\left(u_{p} \psi_{0}+h\left(u_{p}\right)\right)-G\left(u_{p} \psi_{0}+h\left(u_{p}\right)-z\right)\right)\right\|_{2} \\
& \leq C\left\|\langle x\rangle^{\sigma}\left(\left|u_{p} \psi_{0}+h\left(u_{p}\right)\right|^{p}+|z|^{p}\right)|z|\right\|_{2} \\
& =C\left\|\langle x\rangle^{\sigma}\left|u_{p} \psi_{0}+h\left(u_{p}\right)\right|^{p}|z|+\langle x\rangle^{\sigma}|z|^{p+1}\right\|_{2}
\end{aligned}
$$

para alguma constante $C$; da mesma forma, olhando para uma parte da segunda parcela da equação 
(6.22) temos da desigualdade de Cauchy-Schwarz e da estimativa $\left\|\langle x\rangle^{-\sigma} \psi_{0}\right\|_{2} \leq C$

$$
\begin{aligned}
& \left|f_{p}\left(u_{p}, h\left(u_{p}\right)\right)-f_{p}\left(u_{p}, h\left(u_{p}\right)-z\right)\right| \\
= & \left|\left\langle\langle x\rangle^{\sigma}\left(G\left(u_{p} \psi_{0}+h\left(u_{p}\right)\right)-G\left(u_{p} \psi_{0}+h\left(u_{p}\right)-z\right)\right),\langle x\rangle^{-\sigma} \psi_{0}\right\rangle\right| \\
\leq & C\left\|\langle x\rangle^{\sigma}\left(G\left(u_{p} \psi_{0}+h\left(u_{p}\right)\right)-G\left(u_{p} \psi_{0}+h\left(u_{p}\right)-z\right)\right)\right\|_{2} \\
\leq & C\left\|\langle x\rangle^{\sigma}\left|u_{p} \psi_{0}+h\left(u_{p}\right)\right|^{p}|z|+\langle x\rangle^{\sigma}|z|^{p+1}\right\|_{2} .
\end{aligned}
$$

Notemos ainda que

$$
\begin{aligned}
& \left\|D h\left(u_{p}\right)\left(f_{p}\left(u_{p}, h\left(u_{p}\right)\right)-f_{p}\left(u_{p}, h\left(u_{p}\right)-z\right)\right)\right\|_{L_{\sigma}^{2}} \\
\leq & \left\|D h\left(u_{p}\right)\left(f_{p}\left(u_{p}, h\left(u_{p}\right)\right)-f_{p}\left(u_{p}, h\left(u_{p}\right)-z\right)\right)\right\|_{L_{\sigma}^{2} \cap H^{1}} \\
\leq & M\left|f_{p}\left(u_{p}, h\left(u_{p}\right)\right)-f_{p}\left(u_{p}, h\left(u_{p}\right)-z\right)\right| \\
\leq & M C\left\|\langle x\rangle^{\sigma}\left|u_{p} \psi_{0}+h\left(u_{p}\right)\right|^{p}|z|+\langle x\rangle^{\sigma}|z|^{p+1}\right\|_{2} .
\end{aligned}
$$

Substituindo (6.24) e (6.26) na equação (6.22), obtemos

$$
\left\|N\left(u_{p}, z\right)\right\|_{L_{\sigma}^{2}} \leq C\left\|\langle x\rangle^{\sigma}\left|u_{p} \psi_{0}+h\left(u_{p}\right)\right|^{p}|z|+\langle x\rangle^{\sigma}|z|^{p+1}\right\|_{2} .
$$

Agora, usando a equação de Duhamel associada a (6.21) temos que $z(t)$ pode ser escrita para $0<t<T$ como:

$$
z(t)=e^{-i H_{q} t} z(0)-i \lambda \int_{0}^{t} e^{-i H_{q}(t-s)} N\left(u_{p}(s), z(s)\right) d s .
$$

Comecemos estimando a primeira parcela da equação (6.28). Como $z(0) \in \mathscr{R}\left(P_{c}\right)$, temos que $U_{q}(t) z(0)=e^{-i t H_{q}} z(0)$, logo pelo Lema 3.4.1 $(p=2)$, temos para todo $t \neq 0$,

$$
\left\|e^{-i H_{q} t} z(0)\right\|_{L_{-\sigma}^{2}} \leq C\|z(0)\|_{2},
$$

e pelo Teorema 6.1 .1

$$
\left\|e^{-i H_{q} t} z(0)\right\|_{L_{-\sigma}^{2}} \leq C|t|^{-\frac{1}{2}-\beta}\|z(0)\|_{L_{\sigma}^{2}} .
$$

Como

- $L_{\alpha}^{2} \subset L^{1} \cap L^{2}$, desde que $\alpha>1 / 2$;

- $H^{1} \subset L^{2} \cap L^{\infty}$

temos, $L_{\alpha}^{2} \cap H^{1} \subset L^{1} \cap L^{\infty} \subset L^{p_{0}}, p_{0} \geq 1$, assim como $(1+|t|)^{\frac{1}{2}+\beta} \leq c\left(1+|t|^{\frac{1}{2}+\beta}\right)$ temos de (6.30) e (6.29)

$$
\left\|e^{-i H_{q} t} z(0)\right\|_{L_{-\sigma}^{2}} \leq C\langle t\rangle^{-\frac{1}{2}-\beta}\|z(0)\|_{L_{\sigma}^{2} \cap H^{1}}
$$


Agora estimaremos a integral da equação (6.28), e por isto chamaremos

$$
J(t)=\int_{0}^{t} e^{-i H_{q}(t-s)} N\left(u_{p}(s), z(s)\right) d s .
$$

Note que de (6.21), $N\left(u_{p}(s), z(s)\right) \in \mathscr{R}\left(P_{c}\right)$. Então, do Teorema 6.1.1 e da equação (6.27), temos da desigualdade de Minkowski que

$$
\begin{aligned}
\|J(t)\|_{L_{-\sigma}^{2}} \leq & \int_{0}^{t}\left\|e^{-i H_{q}(t-s)} N\left(u_{p}(s), z(s)\right)\right\|_{L_{-\sigma}^{2}} d s \\
\leq & C \int_{0}^{t}\langle t-s\rangle^{-\frac{1}{2}-\beta}\left\|N\left(u_{p}(s), z(s)\right)\right\|_{L_{\sigma}^{2}} d s \\
\leq & C \int_{0}^{t}\langle t-s\rangle^{-\frac{1}{2}-\beta}\left\|\langle x\rangle^{\sigma}\left|u_{p}(s) \psi_{0}+h\left(u_{p}(s)\right)\right|^{p}|z(s)|+\langle x\rangle^{\sigma}|z(s)|^{p+1}\right\|_{2} d s \\
\leq & C \int_{0}^{t}\langle t-s\rangle^{-\frac{1}{2}-\beta}\left\|\langle x\rangle^{\sigma}\left|u_{p}(s) \psi_{0}+h\left(u_{p}(s)\right)\right|^{p}|z(s)|\right\|_{2} d s \\
& +C \int_{0}^{t}\langle t-s\rangle^{-\frac{1}{2}-\beta}\left\|\langle x\rangle^{\sigma}|z(s)|^{p+1}\right\|_{2} d s,
\end{aligned}
$$

porém,

$$
\begin{aligned}
\left\|\langle x\rangle^{\sigma}\left|u_{p} \psi_{0}+h\left(u_{p}\right)\right|^{p}|z|\right\|_{2} & \leq\left\|\langle x\rangle^{2 \sigma}\left|u_{p} \psi_{0}+h\left(u_{p}\right)\right|^{p}\right\|_{\infty}\left\|\langle x\rangle^{-\sigma}|z|\right\|_{2} \\
& =\left\|\langle x\rangle^{\frac{2 \sigma}{p}}\left|u_{p} \psi_{0}+h\left(u_{p}\right)\right|\right\|_{\infty}^{p}\|z\|_{L_{-\sigma}^{2}}
\end{aligned}
$$

Agora, se recordarmos que $\psi(t)=u_{p}(t) \psi_{0}+h\left(u_{p}(t)\right)$ pertence a $\mathcal{W}_{\mu}^{p}$, então vemos da equação (4.3) que

$$
u_{p}(t) \psi_{0}+h\left(u_{p}(t)\right)=e^{i \theta(t)} \psi_{e(t)},
$$

onde $\theta(t)=\operatorname{Arg}\left(u_{p}(t)\right)$ e $e(t)=E\left(\left|u_{p}(t)\right|\right), \operatorname{com} E(r)$ dada pela equação (4.16). Desde que $\|u(t)\|_{2}=$ $\|u(0)\|_{2} \leq \varepsilon$, vemos que como $\psi_{0} \perp u_{c}(t)$ e $\left\|\psi_{0}\right\|_{2}=1$,

$$
\left|\left\langle\psi_{0}, u(t)\right\rangle\right|=\left|\left\langle\psi_{0}, u_{p}(t) \psi_{0}+u_{c}(t)\right\rangle\right|=\left|\left\langle\psi_{0}, u_{p}(t) \psi_{0}\right\rangle+\left\langle\psi_{0}, u_{c}(t)\right\rangle\right|=\left|u_{p}(t)\right|,
$$

$\log 0$

$$
\left|u_{p}(t)\right| \leq\left\|\psi_{0}\right\|_{2}\|u(t)\|_{2}=\|u(t)\|_{2}=\|u(0)\|_{2} \leq \varepsilon,
$$

para todo $t$ onde exista a solução $u(t)$. Pelos Teorema 3.1.2 e Teorema 3.1.4, temos que se $\left\|u_{0}\right\|_{H^{1}} \ll$ $\varepsilon$, então $\|u(t)\|_{H^{1}} \ll c \varepsilon$, para todo $t \in \mathbb{R}$. Mais ainda, como $h$ é de classe $C^{1}$ e $h(0)=0$, $\left\|h\left(u_{p}(t)\right)\right\|_{H^{1}} \leq C\left|u_{p}(t)\right| \leq C \varepsilon, \log 0$

$$
\|z(t)\|_{H^{1}}=\left\|h\left(u_{p}(t)\right)+u_{p}(t) \psi_{0}-u(t)\right\|_{H^{1}} \leq c_{1} \varepsilon
$$

Então, se $\varepsilon<\delta$, pelo Teorema 4.1.1, com $e(s)=E\left(\left|u_{p}(s)\right|\right)=e\left(\left|u_{p}(s)\right|\right),\left|u_{p}(s)\right|<\varepsilon$,

$$
\left\|\langle x\rangle^{\frac{2 \sigma}{p}}\left|u_{p}(s) \psi_{0}+h\left(u_{p}(s)\right)\right|\right\|_{\infty}^{p} \leq C\left\|\langle x\rangle^{\frac{2 \sigma}{p}} \psi_{e(s)}\right\|_{H^{2}(\Omega)}^{p}
$$




$$
\begin{aligned}
& \leq C_{\sigma}\left\|\psi_{e(s)}\right\|_{H^{2}(\Omega)}^{p} \\
& \leq C \nu(\varepsilon)^{p},
\end{aligned}
$$

onde

$$
\nu(\varepsilon)=\sup _{|r| \leq \varepsilon}\left\|\psi_{e(r)}\right\|_{H^{2}(\Omega)} .
$$

Note o sentido de $|r| \leq \varepsilon$ : $e(r)=E(r)$, por abuso de notação, $e(t)=e\left(\left|u_{p}(t)\right|\right)$, sendo $r=\left|u_{p}(t)\right|$, então $|r| \leq \varepsilon$. Sendo assim,

$$
\|J(t)\|_{L_{-\sigma}^{2}} \leq C \int_{0}^{t}\langle t-s\rangle^{-\frac{1}{2}-\beta}\left(\nu(\varepsilon)^{p}\|z(s)\|_{L_{-\sigma}^{2}}+\left\|\langle x\rangle^{\sigma}|z(s)|^{p+1}\right\|_{2}\right) d s .
$$

Agora,

$$
\left\|\langle x\rangle^{\sigma}|z|^{p+1}\right\|_{2} \leq\left\|\langle x\rangle^{2 \sigma}|z|^{p}\right\|_{\infty}\left\|\langle x\rangle^{-\sigma}|z|\right\|_{2} \leq\|\langle x\rangle|z|\|_{\infty}^{p}\|z\|_{L_{-\sigma}^{2}}
$$

Ainda mais, da definição de $z(t)$, das hipotéses no enunciado do Teorema, de $\|u(t)\|_{H^{1}} \leqq C \varepsilon$, e de (6.34) obtemos

$$
\begin{aligned}
\|\langle x\rangle|z|\|_{\infty}^{p} & \leq C\left(\|u(t)\|_{H^{1}}^{p}+\|x u(t)\|_{H^{1}}^{p}\right)+C\left\|\langle x\rangle^{\frac{2 \sigma}{p}} \psi(t)\right\|_{\infty}^{p} \\
& \leq C \varepsilon+C \nu(\varepsilon)^{p} .
\end{aligned}
$$

Então

$$
\left\|\langle x\rangle^{\sigma}|z|^{p+1}\right\|_{2} \leq C\left(\varepsilon+\nu(\varepsilon)^{p}\right)\|z\|_{L_{-\sigma}^{2}} .
$$

Logo,

$$
\|J(t)\|_{L_{-\sigma}^{2}} \leq C \int_{0}^{t}\langle t-s\rangle^{-\frac{1}{2}-\beta}\left(\nu(\varepsilon)^{p}+\varepsilon\right)\|z(s)\|_{L_{-\sigma}^{2}} d s .
$$

Então, das equações (6.31) e (6.36),

$$
\|z(t)\|_{L_{-\sigma}^{2}} \leq C\langle t\rangle^{-\frac{1}{2}-\beta}\|z(0)\|_{L_{\sigma}^{2} \cap H^{1}}+C\left(\nu(\varepsilon)^{p}+\varepsilon\right)|\lambda| \int_{0}^{t}\langle t-s\rangle^{-\frac{1}{2}-\beta}\|z(s)\|_{L_{-\sigma}^{2}} d s .
$$

Definimos para $T>0$,

$$
z_{T}=\sup _{|t| \leq T}\langle t\rangle^{\frac{1}{2}+\beta}\|z(t)\|_{L_{-\sigma}^{2}}
$$

$\log \mathrm{O}$

$$
\begin{aligned}
\|z(t)\|_{L_{-\sigma}^{2}} & \leq C\langle t\rangle^{-\frac{1}{2}-\beta}\|z(0)\|_{L_{\sigma}^{2} \cap H^{1}}+C\left(\nu(\varepsilon)^{p}+\varepsilon\right)|\lambda| \int_{0}^{t}\langle t-s\rangle^{-\frac{1}{2}-\beta}\langle s\rangle^{-\frac{1}{2}-\beta} z_{T} d s \\
& \leq C\langle t\rangle^{-\frac{1}{2}-\beta}\|z(0)\|_{L_{\sigma}^{2} \cap H^{1}}+C\left(\nu(\varepsilon)^{p}+\varepsilon\right)|\lambda|\langle t\rangle^{-\frac{1}{2}-\beta} z_{T},
\end{aligned}
$$


assim teremos

$$
\langle t\rangle^{\frac{1}{2}+\beta}\|z(t)\|_{L_{-\sigma}^{2}} \leq C\left(\|z(0)\|_{L_{\sigma}^{2} \cap H^{1}}+\left(\nu(\varepsilon)^{p}+\varepsilon\right)|\lambda| z_{T}\right)
$$

então

$$
z_{T}\left(1-C\left(\nu(\varepsilon)^{p}+\varepsilon\right)|\lambda|\right) \leq C\|z(0)\|_{L_{\sigma}^{2} \cap H^{1}}
$$

Como para $\varepsilon$ pequeno $\nu(\varepsilon) \rightarrow 0$ (ver Teorema 4.1), temos

$$
z_{T} \leq \frac{C}{1-\left(\nu(\varepsilon)^{p}+\varepsilon\right)|\lambda|}\|z(0)\|_{L_{\sigma}^{2} \cap H^{1}},
$$

portanto, para $t \in[0, T]$,

$$
\langle t\rangle^{\frac{1}{2}+\beta}\|z(t)\|_{L_{-\sigma}^{2}} \leq z_{T} \leq C\|z(0)\|_{L_{\sigma}^{2} \cap H^{1}},
$$

e finalmente como $T$ foi arbitário, temos para $t \geq 0$,

$$
\|z(t)\|_{L_{-\sigma}^{2}} \leq C\langle t\rangle^{-\frac{1}{2}-\beta}\|z(0)\|_{L_{\sigma}^{2} \cap H^{1}}
$$

Uma estimativa similar é obtida para $0>t>-T$. Isto finaliza a prova do teorema.

\subsection{Convergência para uma órbita periódica}

Nessa seção, estabeleceremos que não somente toda solução de (3.1) se aproxima da variedade centro $\mathcal{W}_{\mu}^{p}$, como demonstrado no Teorema 6.2.1, mas também que toda solução se aproxima de uma órbita particular em $\mathcal{W}_{\mu}^{p}$.

Dessa maneira, provaremos nosso principal teorema:

Teorema 6.3.1. Considere o problema (3.1). Suponhamos que $\|x u(t)\|_{H^{1}}$ é suficientemente pequena para todo $t \in \mathbb{R}$. Seja $s>1$ e $\frac{1}{2}<\beta \leq 1$. Se $u(0) \in L_{s+2 \beta}^{2} \cap H_{1}^{1}(\mathbb{R}) \cap H_{2}^{2}(\Omega), 2(s+2 \beta) \leq p$ e $\|u(0)\|_{L_{s+2 \beta}^{2} \cap H^{1}}$ é suficientemente pequena, existem funções diferenciáveis $E(t)=E(r(t)), \theta(t)$ tal que os limites

$$
\begin{aligned}
& E_{ \pm}=\lim _{t \rightarrow \pm \infty} E(t), \\
& \theta_{ \pm}=\lim _{t \rightarrow \pm \infty} \theta(t),
\end{aligned}
$$

existem $e$

$$
\lim _{t \rightarrow \pm \infty}\left\|u(t)-e^{-i\left(\int_{0}^{t} E(s) d s-\theta(t)\right)} \psi_{E(t)}\right\|_{L_{-s-2 \beta}^{2}}=0,
$$

onde $u(t)$ é a solução de (3.1) com condição inicial $u(0)$.

Demonstração: Chame $\sigma=s+2 \beta$. Da seção anterior sabemos que se escrevemos a solução da 
equação $(3.1)$ como $u(t)=u_{p}(t) \psi_{0}+u_{c}(t)$, com $u_{p}(t) \in \mathbb{C}$ e $u_{c}(t) \in \mathscr{R}\left(P_{c}\right)$, então

$$
u(t)=u_{p}(t) \psi_{0}+h\left(u_{p}(t)\right)-z(t)
$$

com $z(t)=h\left(u_{p}(t)\right)-u_{c}(t) \rightarrow 0$ em uma taxa na norma $L_{-\sigma}^{2}$ dada pelo Teorema 6.2.1. Por outro lado, pela equação (3.39) a parte "centro" da solução satisfaz a equação

$$
i \dot{u}_{p}=E_{0} u_{p}-\lambda f_{p}\left(u_{p}, h\left(u_{p}\right)\right)-\lambda Q\left(u_{p}, z\right)
$$

onde $Q\left(u_{p}, z\right)=f_{p}\left(u_{p}, h\left(u_{p}\right)-z\right)-f_{p}\left(u_{p}, h\left(u_{p}\right)\right)$. Consideremos as coordenadas polares $u_{p}(t)=$ $r(t) e^{i \varphi(t)}, \log \mathrm{O}$

$$
\dot{u}_{p}(t)=\dot{r}(t) e^{i \varphi(t)}+i r(t) e^{i \varphi(t)} \dot{\varphi}(t) .
$$

Usando as equações (4.3) e (4.5), obtemos, respectivamente,

$$
\begin{aligned}
u(t) & =\left(u_{p}(t) \psi_{0}+h\left(u_{p}(t)\right)\right)-z(t)=e^{i \varphi(t)} \psi_{E(r(t))}-z(t), \\
\left(E_{0}-E(r(t))\right) r(t) & =\lambda e^{-i \varphi(t)} f_{p}\left(r(t) e^{i \varphi(t)}, h\left(r(t) e^{i \varphi(t)}\right)\right) .
\end{aligned}
$$

Multiplicando (6.40) por $i$ e usando (6.39), temos

$$
E_{0} r(t) e^{i \varphi(t)}-\lambda f_{p}\left(r(t) e^{i \varphi(t)}, h\left(r(t) e^{i \varphi(t)}\right)\right)-\lambda Q\left(r(t) e^{i \varphi(t)}, z(t)\right)=i e^{i \varphi(t)} \dot{r}(t)-e^{i \varphi(t)} r(t) \dot{\varphi}(t)
$$

ou melhor,

$$
E_{0} r(t)-\lambda e^{-i \varphi(t)} f_{p}\left(r(t) e^{i \varphi(t)}, h\left(r(t) e^{i \varphi(t)}\right)\right)-\lambda e^{-i \varphi(t)} Q\left(r(t) e^{i \varphi(t)}, z(t)\right)=i \dot{r}(t)-r(t) \dot{\varphi}(t)
$$

e usando a equação (6.42) obtemos

$$
[E(r(t))] r(t)-\lambda e^{-i \varphi(t)} Q\left(r(t) e^{i \varphi(t)}, z(t)\right)=i \dot{r}(t)-r(t) \dot{\varphi}(t)
$$

assim, tomando a parte imaginária e a parte real, vamos obter o seguinte conjunto de equações

$$
\left\{\begin{array}{l}
\dot{r}=\operatorname{Im}\left(-\lambda e^{-i \varphi} Q\left(r e^{i \varphi}, z\right)\right) \\
\dot{\varphi}=-E(r(t))-\operatorname{Re}\left(-\lambda e^{-i \varphi} r^{-1} Q\left(r e^{i \varphi}, z\right)\right) .
\end{array}\right.
$$

Note que, da equação (6.25),

$$
\begin{aligned}
\left|Q\left(u_{p}(t), z(t)\right)\right| & =\left|f_{p}\left(u_{p}(t), h\left(u_{p}(t)\right)-z(t)\right)-f_{p}\left(u_{p}(t), h\left(u_{p}(t)\right)\right)\right| \\
& \leq C\left\|\langle x\rangle^{\sigma}\left|u_{p} \psi_{0}+h\left(u_{p}\right)\right|^{p}|z|+\langle x\rangle^{\sigma}|z|^{p+1}\right\|_{2},
\end{aligned}
$$

e pelas equações $(6.33)$ e (6.35)

$$
\left|Q\left(u_{p}(t), z(t)\right)\right| \leq C\left(\nu(\varepsilon)^{p}+\varepsilon\right)\|z\|_{L_{-\sigma}^{2}} .
$$


Como $\varepsilon$ é pequeno, pelo Teorema 6.2.1 temos a estimativa

$$
\left|Q\left(u_{p}(t), z(t)\right)\right| \leq C\|z\|_{L_{-\sigma}^{2}} \leq C\langle t\rangle^{-\frac{1}{2}-\beta}\|z(0)\|_{L_{-\sigma}^{2}} .
$$

Integrando a primeira equação em (6.43) temos $r(t)=\int_{0}^{t} \operatorname{Im}\left(-\lambda e^{-i \varphi(s)} Q\left(r(s) e^{i \varphi(s)}, z(s)\right)\right) d s$, pois $r(0)=0$, e $\operatorname{assim} r(t)$ satisfaz

$$
r(t)-r_{ \pm}=O\left(\langle t\rangle^{-\frac{1}{2}-\beta}\right)
$$

quando $t \rightarrow \pm \infty$, com $r_{ \pm}=\lim _{t \rightarrow \pm \infty} r(t)$ (veja Observação 6.3.1). Da mesma maneira, considerando a segunda equação de (6.43), temos

$$
\varphi(t)=-\int_{0}^{t} E(r(s)) d s+\theta(t)
$$

pois $\varphi(0)=0, \operatorname{com} \theta(t)=-\int_{0}^{t} \operatorname{Re}\left(-\lambda e^{-i \varphi(s)}(r(s))^{-1} Q\left(r(s) e^{i \varphi(s)}, z(s)\right)\right) d s$. Assim, usando (6.44), temos que

$$
\theta(t)-\theta_{ \pm}=O\left(\langle t\rangle^{-\frac{1}{2}-\beta}\right)
$$

quando $t \rightarrow \pm \infty$, sendo $\theta_{ \pm}=\lim _{t \rightarrow \pm \infty} \theta(t)$. Combinando (6.41), (6.44), (6.45) e aplicando o Teorema 6.2 .1 , obtemos

$\left\|u(t)-e^{-i\left(\int_{0}^{t} E(s) d s-\theta(t)\right)} \psi_{E(r(t))}\right\|_{L_{-\sigma}^{2}}=\left\|u(t)-e^{i \varphi(t)} \psi_{E(r(t))}\right\|_{L_{-\sigma}^{2}}=\|z(t)\|_{L_{-\sigma}^{2}} \leq C\langle t\rangle^{-\frac{1}{2}-\beta}\|z(0)\|_{L_{-\sigma}^{2}}$.

Então, temos a equação (6.38).

Observação 6.3.1. Para $t>0$,

$$
|r(t)| \leq \int_{0}^{t} \frac{1}{\langle s\rangle^{\frac{1}{2}+\beta}} d s \leq \int_{0}^{t} \frac{1}{(1+s)^{\frac{1}{2}+\beta}} d s,
$$

então $\int_{0}^{\infty} \frac{1}{(1+s)^{\frac{1}{2}+\beta}} d s<\infty$, pois $\frac{1}{2}+\beta>1$, logo $\lim _{t \rightarrow+\infty} r(t)$ existe. 


\section{Aplicação à equação de Schrödinger com um ponto de interação}

Vimos na Seção 6.2, que como a parte não-linear da equação(3.1) é polinomial, tivemos que estudar propriedades da parte dispersiva do grupo $\left\{e^{-i t H_{q}}\right\}_{t \in \mathbb{R}}$ em espaço de Sobolev com peso para assim obter a solução local de (3.1) sobre a parte espectral contínua e portanto deduzir a aproximação à variedade invariante centro $\mathcal{W}_{\mu}^{p}$.

Nesse capítulo, vamos estudar equação de Schrödinger não-linear com potencial singular e com não-linearidade mais gerais, tal que sob certas condições naturais não será necessário trabalhar em espaço de Sobolev com peso. A ideia de mudar o tipo de não-linearidade, surgiu pelo estudo de um artigo publicado por Weder em [45]. Em geral, as soluções para essas equações têm uma componente localizada e uma dispersiva. O bound-state não-linear, que bifurca da solução nula na energia do autovalor de $H_{q}$, define uma variedade invariante centro que consiste de órbitas de soluções localizadas no tempo. Provaremos que todas as soluções com dado inicial pequeno, aproximam-se de uma órbita periódica particular na variedade centro $\mathcal{W}_{\mu}^{p}$, quando $t \rightarrow \pm \infty$. Em geral, as órbitas periódicas são diferentes para $t \rightarrow \pm \infty$. Esses resultados implicam também, que o bound-state não-linear são assintoticamente estável no sentido que, cada solução com dado inicial próximo de um bound-state é assintótico, quando $t \rightarrow \pm \infty$, à uma órbita periódica próxima de um bound-state que são, em geral, diferentes para $t \rightarrow \pm \infty$.

\subsection{NLS- $\delta$ com não linearidades gerais}

Estudaremos a equação de Schrödinger não linear

$$
\left\{\begin{array}{l}
i \frac{\partial u}{\partial t}=H_{q} u+f(x,|u|) \frac{u}{|u|}, \quad(x, t) \in \mathbb{R} \times \mathbb{R}, \\
u(0)=u_{0}
\end{array}\right.
$$

onde $H_{q}=-\frac{1}{2} \Delta+q \delta(x), q<0, \operatorname{com} \delta$ denotando a distribuição Delta de Dirac.

Para cada $x \in \mathbb{R}$ fixo, $f(x, \cdot) \in C^{1}(\mathbb{R}, \mathbb{R}), \frac{\partial}{\partial x} f(x, \cdot) \in C(\mathbb{R}, \mathbb{R}), f(x, 0)=0 \mathrm{e}$

$$
\left|\frac{\partial}{\partial u} f(x, u)\right| \leq C|u|^{p-1}
$$




$$
\left|\frac{\partial}{\partial x} f(x, u)\right| \leq C|u|^{p}, \quad \text { para } \operatorname{algum} p>2
$$

Recordemos que pelo Teorema 2.1.4 para $q<0$, o operador $H_{q}$ tem um único autovalor negativo, $\sigma_{p}\left(H_{q}\right)=\left\{-\frac{q^{2}}{2}\right\}$ com autofunção normalizada $\sqrt{-q} e^{q|x|}$ e seu espectro absolutamente contínuo é $[0, \infty)$.

Inicialmente, quando consideramos a equação de Schrödinger linear; isto é, com $f \equiv 0$,

$$
\left\{\begin{array}{l}
i \frac{\partial u}{\partial t}=H_{q} u, \quad(x, t) \in \mathbb{R} \times \mathbb{R}, \\
u(0)=u_{0}
\end{array}\right.
$$

a equação (7.4) tem uma variedade invariante centro dada por

$$
E_{p}:=\left\{r e^{i \theta} \psi_{0}: r \geq 0,0 \leq \theta<2 \pi\right\}
$$

A variedade invariante, $E_{p}$, consiste de órbitas de soluções periódicas para (7.4) da forma $e^{-i t E_{0}} r e^{i \theta} \psi_{0}$ tais que $r \geq 0$ e $0 \leq \theta<2 \pi$.

Toda solução para (7.4), $u(t)=e^{-i t H} u_{0}$, com $u_{0} \in L^{2}$, pode ser decomposta como segue

$$
e^{-i t H_{q}} u_{0}=e^{-i t E_{0}} P_{p} u_{0}+e^{-i t H_{q}} P_{c} u_{0}
$$

onde $P_{p} u_{0}=\left\langle u_{0}, \psi_{0}\right\rangle \psi_{0}$ e $P_{c}=I-P_{p}$ são a projeção ortogonal sobre o subespaço $\left[\psi_{0}\right]$ e a projeção sobre o espaço de continuidade de $H_{q}, \mathscr{R}\left(H_{q}\right)=P_{c} L^{2}$, respectivamente. Por (7.6) e Lema 3.4.2 toda solução $u$ para (7.4) aproxima-se de uma órbita periódica na variedade invariante centro, pois para qualquer $\sigma>\frac{1}{2}$,

$$
\lim _{t \rightarrow \pm \infty}\left\|u(t)-e^{-i t E_{0}} P_{p} u_{0}\right\|_{L_{-\sigma}^{2}}=\lim _{t \rightarrow \pm \infty}\left\|e^{-i t H_{q}} P_{c} u_{0}\right\|_{L_{-\sigma}^{2}}=0 .
$$

A equação (7.7) nos diz que se o dado inicial $u_{0}=r e^{i \theta} \psi_{0}+u_{c}$ em $L^{2}$, onde $u_{c} \in \mathscr{R}\left(P_{c}\right)$, então a solução para (7.4) é a soma de uma órbita periódica, $e^{-i t E_{0}} r e^{i \theta} \psi_{0}$, e uma solução dispersiva, $e^{-i t H} u_{c}$, cuja energia local converge para zero quanto $t \rightarrow \pm \infty$.

O objetivo deste capítulo é ver que para o caso não-linear vamos obter a mesma situação. A saber, existe uma variedade invariante centro cujas órbitas são de soluções periódicas no tempo, tais que toda solução com dado inicial pequeno para (7.1) aproxima-se de uma órbita particular na variedade centro quando $t \rightarrow \pm \infty$.

Uma solução standing wave para (7.1) é uma solução do tipo $u(x, t)=e^{-i t E} \psi_{E}$, onde $\psi_{E}$ é uma solução do seguinte problema

$$
H_{q} \psi_{E}+f\left(x,\left|\psi_{E}\right|\right) \frac{\psi_{E}}{\left|\psi_{E}\right|}=E \psi_{E}, \quad \psi_{E} \in \mathscr{D}\left(H_{q}\right)
$$

É uma consequência do Teorema de Crandall-Rabinowitz (Teorema 2.3.2) que (7.8) tem exatamente 
uma curva contínua próximas da solução trivial $\psi_{E_{0}}=0$ tal que $\left|E-E_{0}\right|<\mu$, para algum $\mu>0$, e

$$
\lim _{E \rightarrow E_{0}}\left\|\psi_{E}\right\|_{H_{2}(\Omega)}=0
$$

Ainda mais, é possível ver que para alguma constante $C$, temos o decaimento

$$
\left|\psi_{E}(x)\right| \leq C e^{-\sqrt{|E|}|x|}, \quad \text { para }\left|E-E_{0}\right|<\mu .
$$

(veja [8, Teorema 3.2]). Segue de (7.10) que para qualquer $\sigma>0$ existe uma constante $C_{\sigma}$ tal que,

$$
\left\|\psi_{E}\right\|_{L_{\sigma}^{2}} \leq C_{\sigma}, \quad \text { para }\left|E-E_{0}\right|<\mu \text {. }
$$

Agora, a variedade invariante centro para a equação de Schrödinger não linear (7.1) é dada por

$$
\mathcal{W}_{\mu}^{p}=\left\{e^{i \theta} \psi_{E}:\left|E-E_{0}\right|<\mu, 0 \leq \theta<2 \pi\right\} .
$$

As órbitas de $\mathcal{W}_{\mu}^{p}$ são soluções periódicas nos tempo para (7.1) da forma $e^{-i t E} e^{i \theta} \psi_{E}$. Com feito anteriormente, escreveremos $\mathcal{W}_{\mu}^{p}$ como o gráfico de uma função do subespaço $\left[\psi_{0}\right]$ em seu complemento ortogonal $\mathscr{R}\left(P_{c}\right)$. Pelo Teorema 4.2.1, segue que existe $\delta>0$ e uma função de classe $C^{1}, h$, de $\left\{u_{p} \in \mathbb{C}:\left|u_{p}\right|<\delta\right\}$ em $\mathscr{R}\left(P_{c}\right) \cap H_{1} \cap L_{\sigma}^{2}, \sigma>\frac{1}{2}$, tal que,

$$
\mathcal{W}_{\mu}^{p}=\left\{\psi: \psi=u_{p} \psi_{0}+h\left(u_{p}\right) ;\left|u_{p}\right|<\delta\right\}
$$

Além disso, $h(0)=0$ e $h\left(e^{i \theta} u_{p}\right)=e^{i \theta} h\left(u_{p}\right)$.

Seja $F: \mathbb{R} \times \mathbb{R} \rightarrow \mathbb{R}$ dada por $F(x, u)=\int_{0}^{u} f(x, v) d v$. Já temos que $f(x, 0)=0$. Segue de (7.3) que para todo $K>0$ existe $L(K)>0$ tal que

$$
|f(x, u)-f(x, v)| \leq L(K)|u-v|
$$

para todo $x \in \mathbb{R}$ e para todo $|u|,|v| \leq K$ e $L \in C([0, \infty)) . \quad f$ estende-se para o plano complexo definida por

$$
f(x, u)=\frac{u}{|u|} f(x,|u|), \quad \text { para todo } u \in \mathbb{C}, u \neq 0 .
$$

Logo, seja

$$
g(u)(x)=f(x, u(x)), x \in \mathbb{R}
$$

para toda $u: \mathbb{R} \rightarrow \mathbb{C}$ e

$$
G(u)=\int_{\mathbb{R}} F(x,|u(x)|) d x
$$

para toda $u: \mathbb{R} \rightarrow \mathbb{C}$ tal que $F(\cdot, u(\cdot)) \in L^{1}(\mathbb{R})$.

Observação 7.1.1. Por [11, Proposição 3.2.5] temos as seguintes propriedades

- $G \in C^{1}\left(H^{1}(\mathbb{R}), \mathbb{R}\right), g \in C\left(H^{1}(\mathbb{R}), H^{-1}(\mathbb{R})\right)$ e $G^{\prime}=g$

- $g \in C\left(L^{2}(\mathbb{R}), L^{2}(\mathbb{R})\right.$; 
- para todo $M>0$, existe $C(M)<\infty$ tal que $\|g(u)-g(v)\|_{2} \leq C(M)\|u-v\|_{2}$ para todo $u, v \in H^{1}(\mathbb{R}) \operatorname{com}\|u\|_{H^{1}},\|v\|_{H^{1}} \leq M$;

- $\operatorname{Im} g(u) \bar{u}=0$ para todo $u \in H^{1}$

Segue de (7.3) e $f(x, 0)=0$, que $|f(x, u)| \leq C|u|^{p}$ e

$$
|F(x,|u|)| \leq C|u|^{p+1}
$$

para alguma constante $C$. De [11, Teorema 3.5.1, Corolário 3.5.3] temos que existe um $\rho>0$ tal que o problema de valor inicial (7.1) tem uma única solução em $C\left(\mathbb{R}, H^{1}\right)$ para todo $u_{0} \in H^{1}$ tal que $\left\|u_{0}\right\|_{H^{1}}<\rho$. Se mais ainda,

$$
F(x,|u|) \leq C\left(1+|u|^{\delta-1}\right)|u|^{2}, \text { para algum } 1<\delta<5,
$$

então, por [11, Colorário 3.5.2] o problema de valor inicial (7.1) tem uma única solução em $C\left(\mathbb{R}, H^{1}\right)$ para todo $u_{0} \in H^{1}$. Em ambos os casos (onde $F$ satisfaz (7.18) ou (7.19)), a norma em $L^{2}$ e a energia são quantidades conservadas

$$
\begin{aligned}
\|u(t)\|_{2} & =\left\|u_{0}\right\|_{2} \\
\frac{1}{2}\left\|u_{x}(x, t)\right\|_{2}^{2}-q|u(0, t)|^{2}+\int_{\mathbb{R}} F(x,|u(x, t)|) d x & =\frac{1}{2}\left\|\left(u_{0}\right)_{x}\right\|_{2}^{2}-q\left|u_{0}(0)\right|^{2}+\int_{\mathbb{R}} F\left(x,\left|u_{0}\right|\right) d x .
\end{aligned}
$$

Mais ainda, é fácil ver que, para todo $\epsilon>0$ existe um $\nu>0$ tal que se $\left\|u_{0}\right\|_{H^{1}}<\nu$, então

$$
\|u(t)\|_{H^{1}}<\epsilon, \quad t \in \mathbb{R}
$$

Observação 7.1.2. Se em (7.22) $\delta=5$, temos a existência global desde que $\left\|u_{0}\right\|_{2}$ é suficientemente pequeno. (veja Seção 3.1)

\subsection{Estabilidade assintótica para bound-states não-lineares asso- ciados a (7.8)}

Teorema 7.2.1. Suponhamos que para cada $x \in \mathbb{R}, f(x, \cdot) \in C^{1}(\mathbb{R}, \mathbb{R}), \frac{\partial}{\partial x} f(x, \cdot) \in C(\mathbb{R}, \mathbb{R}), f(x, 0)=$ 0 e, para algum $p>2$,

$$
\left|\frac{\partial}{\partial u} f(x, u)\right| \leq q(x)|u|^{p-1}
$$

onde $(1+|x|)^{2 s+4 \beta} q(x) \in L^{\infty}(\mathbb{R})$, para algum $s>1$ e $1 / 2<\beta \leq 1$. Mais ainda,

$$
\left|\frac{\partial}{\partial x} f(x, u)\right| \leq C|u|^{p}
$$


Então, existe um $\eta>0$, tal que para todo $u_{0} \in H^{1}(\mathbb{R}) \cap L_{s+2 \beta}^{2}(\mathbb{R})$ com $\left\|u_{0}\right\|_{H^{1}}<\eta$, existem funções, $E(t)$ e $\theta(t)$, em $C^{1}(\mathbb{R}, \mathbb{R})$, tal que para alguma constante $C$ (independe do tempo),

$$
\left\|u(t)-e^{-i \int_{0}^{t} E(\rho) d \rho} e^{i \theta(t)} \psi_{E(t)}\right\|_{L_{-s-2 \beta}^{2}} \leq C\langle t\rangle^{-1 / 2-\beta}\left\|P_{c} u_{0}-h\left(\left\langle u_{0}, \psi_{0}\right\rangle\right)\right\|_{L_{s+2 \beta}^{2}},
$$

onde u(t) é a solução para (7.1) com dado inicial $u_{0}$. Mais ainda, os seguintes limites existem,

$$
\lim _{t \rightarrow \pm \infty} E(t)=E_{ \pm} ; \lim _{t \rightarrow \pm \infty} \theta(t)=\theta_{ \pm}
$$

Observação 7.2.1. A equação (7.25) nos diz que u converge para a órbita periódica de $e^{i \theta_{ \pm}} \psi_{E_{ \pm}}$. Note que a parte dispersiva, $u(t)-e^{-i \int_{0}^{t} E(\rho) d \rho} e^{i \theta(t)} \psi_{E(t)}$, converge para zero em $L_{-s-2 \beta}^{2}$, quando $t \rightarrow \pm \infty$, com a mesma taxa que a solução dispersiva da equação de Schrödinger linear (7.4) (veja o Teorema 6.1.1).

Demonstração: Usando as projeções ortogonais $P_{p}$ e $P_{c}$ (veja Seção 2.28), então, (7.1) é equivalente ao seguinte sistema,

$$
\left\{\begin{aligned}
i \frac{d}{d t} u_{p} & =E_{0} u_{p}+g_{p}\left(u_{p}, u_{c}\right) ; \\
i \frac{\partial}{\partial t} u_{c} & =H_{q} u_{c}+g_{c}\left(u_{p}, u_{c}\right),
\end{aligned}\right.
$$

onde, denotando $g(x, u)=f(x,|u|) \frac{u}{|u|}$, temos

$$
\begin{aligned}
& g_{p}\left(u_{p}, u_{c}\right)=P_{p} g\left(x, u_{p} \psi_{0}+u_{c}\right)=\left\langle g\left(x, u_{p} \psi_{0}+u_{c}\right), \psi_{0}\right\rangle \psi_{0} ; \\
& g_{c}\left(u_{p}, u_{c}\right)=P_{c} g\left(x, u_{p} \psi_{0}+u_{c}\right) .
\end{aligned}
$$

Qualquer ponto na variedade centro $\mathcal{W}_{\mu}^{p}$ é escrita como $e^{i \theta} \psi_{E}=u_{p} \psi_{0}+h\left(u_{p}\right), h\left(u_{p}\right) \in \mathscr{R}\left(P_{c}\right)$, onde $u_{p}, h\left(u_{p}\right)$ são as soluções do seguinte sistema

$$
\left\{\begin{array}{l}
E_{0}-E=-\frac{g_{p}\left(u_{p}, h\left(u_{p}\right)\right)}{u_{p}} ; \\
h\left(u_{p}\right)=-\left(H_{q}-E\right)^{-1} g_{c}\left(u_{p}, h\left(u_{p}\right)\right) .
\end{array}\right.
$$

Vamos considerar $\psi(t)=u_{p}(t) \psi_{0}+h\left(u_{p}(t)\right) \in \mathcal{W}_{\mu}^{p}$. Provaremos que a diferença $z(t)=$ $u(t)-\psi(t)=u_{c}(t)-h\left(u_{p}(t)\right)$ satisfaz a estimativa

$$
\|z(t)\|_{L_{-s_{1}}^{2}} \leq C\langle t\rangle^{-1 / 2-\beta}\|z(0)\|_{L_{s_{1}}^{2}},
$$

onde $s_{1}=s+2 \beta$. Por $(7.27), z(t)$ é uma solução da seguinte equação

$$
i \frac{\partial}{\partial t} z(t)=H_{q} z(t)+N\left(u_{p}(t), z(t)\right),
$$


onde

$$
N\left(u_{p}, u_{c}\right)=g_{c}\left(u_{p}, h\left(u_{p}\right)+z\right)-g_{c}\left(u_{p}, h\left(u_{p}\right)\right)-(D h)\left(u_{p}\right)\left[g_{p}\left(u_{p}, h\left(u_{p}\right)+z\right)-g_{p}\left(u_{p}, h\left(u_{p}\right)\right)\right]
$$

onde $(D h)$ é a derivada de Frechét de $h$. Para verificar (7.32) devemos mostrar que

$$
(D h)\left(u_{p}\right)\left[E_{0} u_{p}+g_{p}\left(u_{p}, h\left(u_{p}\right)\right)\right]=H_{q} h\left(u_{p}\right)+g_{c}\left(u_{p}, h\left(u_{p}\right)\right)
$$

De fato, seja $t_{0} \in \mathbb{R}$. Denotamos $E=E\left(u_{p}\left(t_{0}\right)\right)$. Note que por (7.29), $\left[e^{-i t E} u_{p}\left(t_{0}\right), h\left(e^{-i t E} u_{p}\left(t_{0}\right)\right)\right]$ é uma solução para (7.27) (já vimos que $h\left(e^{-i t E} u_{p}\right)=e^{-i t E} h\left(u_{p}\right)$ ). Então, usando a equação de $u_{p}$ em (7.27),

$$
i \frac{\partial}{\partial t} h\left(e^{-i t E} u_{p}\left(t_{0}\right)\right)=(D h)\left(e^{-i t E} u_{p}\left(t_{0}\right)\right)\left[E_{0} e^{-i t E} u_{p}\left(t_{0}\right)+e^{-i t E} g_{p}\left(u_{p}\left(t_{0}\right), h\left(u_{p}\left(t_{0}\right)\right)\right)\right] .
$$

Mais ainda, pela equação de $u_{c}$ em (7.27),

$$
i \frac{\partial}{\partial t} h\left(e^{-i t E} u_{p}\left(t_{0}\right)\right)=H_{q} h\left(e^{-i t E} u_{p}\left(t_{0}\right)\right)+e^{-i t E} g_{c}\left(u_{p}\left(t_{0}\right), h\left(u_{p}\left(t_{0}\right)\right)\right)
$$

A equação (7.33) segue-se fazendo $t=0 \mathrm{em}$ (7.34) e (7.35). Por (7.23), $|g(x, u+z)-g(x, u)| \leq$ $C q(x)\left(|u|^{(p-1)}+|z|^{(p-1)}\right)|z|$, e temos, pelo Teorema de Sobolev [1],

$$
\begin{aligned}
& \left\|g\left(x, u_{p} \psi_{0}+h\left(u_{p}\right)\right)-g\left(x, u_{p} \psi_{0}+h\left(u_{p}\right)+z\right)\right\|_{L_{s_{1}}^{2}} \\
\leq & C\left\|(1+|x|)^{2 s_{1}} q(x)\right\|_{\infty}\left(\left\|\left(u_{p} \psi_{0}+h\left(u_{p}\right)\left\|_{H_{1}}^{(p-1)}+\right\| z \|_{H_{1}}^{(p-1)}\right)\right\| z \|_{L_{-s_{1}}^{2}} .\right.
\end{aligned}
$$

Por (7.10), $P_{p}$ e $P_{c}=I-P_{p}$ são operadores limitados em $L_{s}^{2}, s \in \mathbb{R}$, e segue de (7.36) that

$$
\begin{gathered}
\left\|g_{p}\left(u_{p}, h\left(u_{p}\right)\right)-g_{p}\left(u_{p}, h\left(u_{p}\right)+z\right)\right\|_{L_{s_{1}}^{2}} \leq C\left(\left\|u_{p} \psi_{0}+h\left(u_{p}\right)\right\|_{H^{1}}^{(p-1)}+\|z\|_{H^{1}}^{(p-1)}\right)\|z\|_{L_{-s_{1}}^{2}} \\
\left\|g_{c}\left(u_{p}, h\left(u_{p}\right)\right)-g_{c}\left(u_{p}, h\left(u_{p}\right)+z\right)\right\|_{L_{s_{1}}^{2}} \leq C\left(\left\|u_{p} \psi_{0}+h\left(u_{p}\right)\right\|_{H^{1}}^{(p-1)}+\|z\|_{H^{1}}^{(p-1)}\right)\|z\|_{L_{-s_{1}}^{2}}
\end{gathered}
$$

Por (7.22) dado qualquer any $\epsilon_{1}>0$ podemos escolher $\eta$ suficientemente pequeno que se $\left\|u_{0}\right\|_{H^{1}}<\eta$, temos $\left|u_{p}(t)\right|=\left|\left\langle u(t), \psi_{0}\right\rangle\right| \leq\|u(t)\|_{H^{1}}<\epsilon_{1}$. Além disso, como $h$ é de classe $C^{1} \mathrm{e}$ $h(0)=0$,

$$
\left\|h\left(u_{p}(t)\right)\right\|_{H^{1}} \leq C\left|u_{p}\right| \leq C \epsilon_{1}
$$

e concluímos

$$
\|z(t)\|_{H^{1}} \leq C \epsilon_{1}
$$

Por $(7.32),(7.36),(7.37)$ e (7.38),

$$
\left\|N\left(u_{p}(t), z(t)\right)\right\|_{L_{s_{1}}^{2}} \leq C \epsilon_{1}\|z(t)\|_{L_{-s_{1}}^{2}}, \text { se }\left\|u_{0}\right\|_{H_{1}}<\eta
$$


Escrevemos (7.31) como uma equação integral

$$
z(t)=e^{-i t H_{q}} z(0)-i \int_{0}^{t} e^{-i(t-s) H_{q}} N\left(u_{p}(s), z(s)\right) d s .
$$

Denotaremos $z_{T}=\max _{|t| \leq T}\langle t\rangle^{1 / 2+\beta}\|z(t)\|_{L_{-s_{1}}^{2}}$. Pelo Teorema 6.1.1 e (7.41), para $|t| \leq T$,

$$
\begin{aligned}
\|z(t)\|_{L_{-1 / 2-\beta}^{2}} & \leq C\langle t\rangle^{-1 / 2-\beta}\|z(0)\|_{L_{s_{1}}^{2}}+C \epsilon_{1} \int_{0}^{t}\langle t-s\rangle^{-1 / 2-\beta}\langle s\rangle^{-1 / 2-\beta} z_{T} d s \\
& \leq C\langle t\rangle^{-1 / 2-\beta}\left[\|z(0)\|_{L_{s_{1}}^{2}}+C \epsilon_{1} z_{T}\right] .
\end{aligned}
$$

Fazendo $\eta$ suficientemente pequeno tal que que $C \epsilon_{1}<\frac{1}{2}$, obtemos

$$
z_{T} \leq C\|z(0)\|_{L_{s_{1}}^{2}}
$$

e como a constante $C$ é independente de $T$ a equação (7.30) segue. Seguindo os passos da Seção 6.3, e usando a equação (7.30), obtemos (7.25).

\subsection{Considerações}

1. Note que da condição $(7.23)$ e $(1+|x|)^{2 s+4 \beta} q(x) \in L^{\infty}(\mathbb{R})$, obtemos a condição (7.2). Assim, a solução $u(t) \in C\left(\mathbb{R} ; H^{1}(\mathbb{R})\right)$ e, portanto, $z(t) \in C\left(\mathbb{R} ; H^{1}(\mathbb{R})\right)$.

2. O modelo com não-linearidade não-homogênea

$$
i \frac{\partial u}{\partial t}=H_{q} u+K(x)|u|^{p-1} u, \quad p>1
$$

com $K(x)$ satisfazendo $(1+|x|)^{2 s+4 \beta} K(x) \in L^{\infty}(\mathbb{R})$, encaixa-se no modelo geral (7.1).

3. O modelo (7.45) surge, no sentido físico, no artigo "Optical guiding of laser beam in nonuniform plasma" publicado por Gill em [19]. 


\section{Futuros Trabalhos}

Em todo nosso trabalho, estudamos a equação de Schrödinger não-linear quando o potencial é a distribuição delta de Dirac.

Já existem na literatura estudos quando no operador $H_{q}$ substituímos o potencial $\delta$ por $\delta^{\prime}$ (derivada de $\delta$ ) ou soma de duas $\delta$-interação. Sendo assim, denotaremos os operadores

$$
\begin{aligned}
& H_{\alpha}=-\frac{1}{2} \frac{d^{2}}{d x^{2}}+\alpha \delta^{\prime}(x), \\
& H_{\beta}=-\frac{1}{2} \frac{d^{2}}{d x^{2}}+\beta(\delta(x+a)+\delta(x-a)),
\end{aligned}
$$

onde $a \in \mathbb{R}, a>0$, que determinam, respectivamente, os grupos unitários,

$$
\begin{aligned}
& U_{\alpha}(t)=e^{-i t H_{\alpha}}, \\
& U_{\beta}(t)=e^{-i t H_{\beta}} .
\end{aligned}
$$

Apresentamos algumas propriedades já conhecidos em ambas situações. Consideremos $A=-\frac{d^{2}}{d x^{2}}$ sobre $L^{2}(\mathbb{R})$ com o domínio $\mathscr{D}(A)=H^{2}(\mathbb{R})$.

\subsection{Operador $H_{\alpha}$}

$$
\begin{aligned}
& \text { Seja }-\Delta_{\alpha}=-\frac{d^{2}}{d x^{2}}+\alpha \delta^{\prime}(x) \text { O operador de restrição } \\
&\left\{\begin{aligned}
A_{1} & \equiv A \mathscr{D}_{\left(A_{1}\right)}, \\
\mathscr{D}\left(A_{1}\right) & =\left\{g \in \mathscr{D}(A) \mid g(0)=g^{\prime}(0)=0\right\} ;
\end{aligned}\right.
\end{aligned}
$$

o adjunto de $A_{1}$ é dado por (Ver [3, Capítulo I.4])

$$
\left\{\begin{aligned}
A_{1}^{*} & =-\frac{d^{2}}{d x^{2}} \\
\mathscr{D}\left(A_{1}^{*}\right) & =H^{2}(\mathbb{R}-\{0\}) .
\end{aligned}\right.
$$

Além disso, $A_{1}$ tem índice de deficiência $(2,2)$.

Por Albeverio et al. [3], temos a seguinte família a um parâmetro de extensões autoadjuntas de $A_{1}$ e propriedades espectrais para $-\Delta_{\alpha}$, como seguem 
Teorema 8.1.1. Todas as extensões autoadjuntas $-\Delta_{\alpha}$ de $A_{1}$, onde $-\infty<\alpha \leq \infty$ são dadas por

$$
\left\{\begin{array}{l}
-\Delta_{\alpha}=-\frac{d^{2}}{d x^{2}}, \\
\mathscr{D}\left(-\Delta_{\alpha}\right)=\left\{g \in H^{2}(\mathbb{R}-\{0\}) \mid g^{\prime}(0+)=g^{\prime}(0-), g(0+)-g(0-)=\alpha g^{\prime}(0)\right\} .
\end{array}\right.
$$

Se $\alpha=0$, obtemos o operador de Laplace no espaço $L^{2}(\mathbb{R})$, ou seja,

$$
-\Delta=-\frac{d^{2}}{d x^{2}}, \quad \mathscr{D}(-\Delta)=H^{2}(\mathbb{R})
$$

enquanto se $\alpha=\infty$, a reta real é dividida em dois intervalos $(-\infty, 0)$ e $(0, \infty)$, isto acontece devido a aparição da condição de fronteira do tipo Neumann no ponto 0, isto é

$$
\left\{\begin{aligned}
\mathscr{D}\left(-\Delta_{\infty}\right) & =\left\{g \in H^{2}(\mathbb{R}-\{0\}) \mid g^{\prime}(0+)=g^{\prime}(0-)=0\right\}=\mathscr{D}\left(-\Delta_{D_{-}}\right) \oplus \mathscr{D}\left(-\Delta_{D_{+}}\right), \\
-\Delta_{\infty} & =\left(-\Delta_{D_{-}}\right) \oplus\left(-\Delta_{D_{+}}\right),
\end{aligned}\right.
$$

onde $\left(-\Delta_{D_{ \pm}}\right)$denota o Laplaciano de Neumann sobre $(-\infty, 0),(0, \infty)$, respectivamente,com $\mathscr{D}\left(-\Delta_{D_{-}}\right)=\left\{H_{0}^{2}((-\infty, 0)): g^{\prime}(0-)=0\right\}$ e $\mathscr{D}\left(-\Delta_{D_{+}}\right)=\left\{H_{0}^{2}((0, \infty)): g^{\prime}(0+)=0\right\}$.

Teorema 8.1.2. Seja $-\infty<\alpha \leq \infty$. O espectro essencial de $-\Delta_{\alpha}=-\frac{d^{2}}{d x^{2}}+\alpha \delta^{\prime}$ é o eixo real não negativo, $\sigma_{\text {ess }}\left(-\Delta_{\alpha}\right)=[0, \infty)$. Se $-\infty<\alpha<0,-\Delta_{\alpha}$ tem precisamente um autovalor simples e negativo, isto é, $\sigma_{p}\left(-\Delta_{\alpha}\right)=\left\{-\frac{4}{\alpha^{2}}\right\}$, com $\psi_{\alpha}(x)=\operatorname{sign}(x) \sqrt{-\frac{\alpha}{8}} e^{\frac{2}{\alpha}}|x|$ sendo sua autofunção normalizada. Se $\alpha \geq 0$ ou $\alpha=\infty,-\Delta_{\alpha}$ não tem autovalores, $\sigma_{p}\left(-\Delta_{\alpha}\right)=\emptyset$.

Logo, temos o seguinte resumo para $\alpha<0$ :

$$
H_{\alpha} \equiv-\frac{1}{2} \Delta+\alpha \delta^{\prime}(x)=\frac{1}{2}\left(-\Delta+2 \alpha \delta^{\prime}(x)\right),
$$

tem um único autovalor negativo, $\sigma_{p}\left(H_{\alpha}\right)=\left\{-\frac{2}{\alpha^{2}}\right\}$ com autofunção normalizada $\frac{\sqrt{-\alpha}}{2} e^{\frac{1}{q}|x|}$; além disso, $\mathscr{D}\left(H_{\alpha}\right)=\left\{u \in \cap H^{2}(\mathbb{R}-\{0\}) \mid u^{\prime}(0+)=u^{\prime}(0-), u^{\prime}(0+)-u^{\prime}(0-)=2 \alpha u^{\prime}(0)\right\}$.

\subsection{Operador $H_{\beta}$}

$$
\begin{aligned}
\text { Seja }-\Delta_{\beta}=-\frac{d^{2}}{d x^{2}}+\beta(\delta(x-a)+\delta(x+a)) \text {. O operador de restrição } \\
\qquad \begin{aligned}
A_{2} & \left.\equiv A\right|_{\mathscr{D}\left(A_{2}\right)} \\
\mathscr{D}\left(A_{2}\right) & =\{g \in \mathscr{D}(A) \mid g( \pm a)=0\} ;
\end{aligned}
\end{aligned}
$$

o adjunto de $A_{2}$ é dado por (Ver [3, Seção II.2.1])

$$
\left\{\begin{aligned}
A_{2}^{*} & =-\frac{d^{2}}{d x^{2}} \\
\mathscr{D}\left(A_{2}^{*}\right) & =H^{1}(\mathbb{R}) \cap H^{2}(\mathbb{R}-\{ \pm a\})
\end{aligned}\right.
$$


Além disso, $A_{2}$ tem índice de deficiência $(2,2)$.

Por Albeverio et al. [3], temos a seguinte família a um parâmetro de extensões autoadjuntas de $A_{2}$ e propriedades espectrais para $-\Delta_{\beta}$, como seguem

Teorema 8.2.1. Todas as extensões autoadjuntas $-\Delta_{\beta}$ de $A_{2}$, onde $-\infty<\beta \leq \infty$ são dadas por

$$
\left\{\begin{array}{l}
-\Delta_{\beta}=-\frac{d^{2}}{d x^{2}} \\
\mathscr{D}\left(-\Delta_{\beta}\right)=\left\{g \in H^{1}(\mathbb{R}) \cap H^{2}(\mathbb{R}-\{ \pm a\}) \mid g^{\prime}( \pm a+)-g^{\prime}( \pm a-)=\beta g( \pm a)\right\} .
\end{array}\right.
$$

Se $\beta=0$, obtemos o operador de Laplace no espaço $L^{2}(\mathbb{R})$, ou seja,

$$
-\Delta=-\frac{d^{2}}{d x^{2}}, \quad \mathscr{D}(-\Delta)=H^{2}(\mathbb{R}),
$$

enquanto se $\beta=\infty$, a reta real é dividida em dois intervalos $(-\infty, 0)$ e $(0, \infty)$, isto acontece devido a aparição da condição de fronteira do tipo Dirichelt no ponto \pm a, isto é

$$
\left.\mathscr{D}\left(-\Delta_{\infty}\right)=\left\{g \in H^{1}(\mathbb{R}) \cap H^{2}(\mathbb{R}-\{ \pm a\})\right) \mid g( \pm a+)=g( \pm a-)=0\right\}
$$

Teorema 8.2.2. Seja $-\infty<\beta \leq \infty$. O espectro essencial de $-\Delta_{\beta}=-\frac{d^{2}}{d x^{2}}+\beta \delta^{\prime}$ é o eixo real não negativo, $\sigma_{\text {ess }}\left(-\Delta_{\beta}\right)=[0, \infty)$. Se $-\infty<\beta<0$, então o espectro discreto de $-\Delta_{\beta}, \sigma_{p}\left(-\Delta_{\beta}\right)$, consiste de autovalores negativos $\gamma$ dados pela equação implícita

$$
(-2 i \eta+\beta)^{2}=\beta^{2} e^{4 i \eta \beta}, \quad \eta=\sqrt{\gamma}, \quad \operatorname{Im} \eta>0 .
$$

Se $\beta \geq 0$ ou $\beta=\infty,-\Delta_{\beta}$ não tem autovalores, $\sigma_{p}\left(-\Delta_{\beta}\right)=\emptyset$.

\subsection{Planos}

Tanto para $U_{\alpha}$ quanto para $U_{\beta}$, Angulo \& Ferreira em [5, Proposição 4.4] mostram estimativas dispersivas para os grupos.

Observamos que se $\alpha<0$, obtemos para o operador $H_{\alpha}$ um autovalor. Desta forma, será possível decompor o espaço $L^{2}$ como uma soma direta como fizemos no nosso estudo. Além disso, o núcleo de $H_{\alpha}+\frac{2}{\alpha^{2}}$ é unidimensional. Logo, trabalhando com a equação de Schrödinger não-linear para esse caso, acreditamos que na possibilidade de obter os mesmos resultados apresentados nessa tese.

Mais ainda, se $\beta<0$, o operador $H_{\beta}$ possui um ou dois autovalores (veja [5, Teorema 3.2]). Mais uma vez, sobre o problema não-linear, acreditamos que podemos obtermos também os resultados apresentados nessa tese. 


\section{Soluções da NLS- $\delta$}

\section{A.1 Equação solução de (1.3)}

\section{A.1.1 $q=0$}

Queremos obter uma solução para a equação

$$
i u_{t}+\frac{1}{2} u_{x x}+\lambda|u|^{p} u=0
$$

da forma

$$
u_{s}(x, t)=e^{i \omega t} \phi(x-v t),
$$

onde $\lambda>0$ e $v$ é a velocidade da onda e $\phi: \mathbb{R} \rightarrow \mathbb{C}$. Assim teremos

$$
-\omega \phi(x-v t)-i \phi^{\prime}(x-v t) v+\frac{1}{2} \phi^{\prime \prime}(x-v t)+\lambda|\phi(x-v t)|^{p} \phi(x-v t)=0 .
$$

Afim de cancelar $\phi^{\prime}$, vamos escrever $\phi(\xi)=e^{a \xi i} \varphi(\xi)$, onde $\xi=x-v t$ e $\varphi: \mathbb{R} \rightarrow \mathbb{R}$, com $\varphi(\xi) \rightarrow 0$, quando $|\xi| \rightarrow \pm \infty$. Logo,

$$
\left(-\omega+v a-\frac{a^{2}}{2}\right) \varphi(\xi)+(-i v+i a) \varphi^{\prime}(\xi)+\frac{1}{2} \varphi^{\prime \prime}(\xi)+\lambda(\varphi(\xi))^{p+1}=0 .
$$

Então, considerando $a=v$ e chamando $\alpha=\omega-\frac{v^{2}}{2}$, obteremos

$$
-\alpha \varphi(\xi)+\frac{1}{2} \varphi^{\prime \prime}(\xi)+\lambda(\varphi(\xi))^{p+1}=0 .
$$

Sabemos que pode existir um perfil $\varphi$ satisfazendo (A.1) e $\varphi(\xi) \rightarrow 0$, quando $|\xi| \rightarrow \pm \infty$, com $\alpha>0$. Se multiplicarmos a equação (A.1) por $\varphi^{\prime}(\xi)$, teremos

$$
\frac{d}{d \xi}\left(-\frac{\alpha}{2}[\varphi(\xi)]^{2}+\frac{1}{4}\left[\varphi^{\prime}(\xi)\right]^{2}+\frac{\lambda}{p+2}[\varphi(\xi)]^{p+2}\right)=0
$$

ou seja,

$$
(p+2)\left[\varphi^{\prime}\right]^{2}=2 \alpha(p+2)[\varphi]^{2}-4 \lambda[\varphi]^{p+2}
$$


Chamando $\varphi=\frac{1}{g^{\frac{2}{p}}}$, como $\varphi^{\prime}=-\frac{2}{p} \frac{1}{g^{\frac{p+2}{p}}} g^{\prime}$, temos

$$
\frac{4(p+2)}{p^{2}} \frac{1}{g^{\frac{2(p+2)}{p}}}\left[g^{\prime}\right]^{2}=2 \alpha(p+2) \frac{1}{g^{\frac{4}{p}}}-4 \lambda \frac{1}{g^{\frac{2(p+2)}{p}}} \Leftrightarrow\left[\sqrt{\frac{\alpha}{2}} \sqrt{\frac{p+2}{\lambda}} g\right]^{2}-\left[\frac{1}{p} \sqrt{\frac{p+2}{\lambda}} g^{\prime}\right]^{2}=1 .
$$

Recordemos que $\cosh ^{2}(\theta)-\sinh ^{2}(\theta)=1$ e que $\frac{d}{d \theta} \cosh (\theta)=\sinh (\theta)$. Logo,

$$
g(\xi)=\sqrt{\frac{2}{\alpha}} \sqrt{\frac{\lambda}{p+2}} \cosh \left[p \sqrt{\frac{\alpha}{2}} \xi\right] .
$$

Então,

$$
\varphi(\xi)=\left(\frac{\alpha}{2}\right)^{\frac{1}{p}}\left[\frac{p+2}{\lambda} \operatorname{sech}^{2}\left(p \sqrt{\frac{\alpha}{2}} \xi\right)\right]^{\frac{1}{p}}
$$

onde $\alpha=\omega-\frac{v^{2}}{2}$.

Portanto,

$$
\begin{aligned}
u(x, t) & =e^{i \omega t} e^{i v(x-v t)}\left(\frac{\alpha}{2}\right)^{\frac{1}{p}}\left[\frac{p+2}{\lambda} \operatorname{sech}^{2}\left(p \sqrt{\frac{\alpha}{2}} \xi\right)\right]^{\frac{1}{p}} \\
& =\left(\frac{\alpha}{2}\right)^{\frac{1}{p}} e^{i(v x-\varpi t)}\left[\frac{p+2}{\lambda} \operatorname{sech}^{2}\left(p \sqrt{\frac{\alpha}{2}}(x-v t)\right)\right]^{\frac{1}{p}}
\end{aligned}
$$

onde $\varpi=-\left(v^{2}-\omega\right)$ é a frequência temporal e $\alpha=\omega-\frac{v^{2}}{2}$.

Observação A.1.1. Suponhamos que $\lambda<0$, então seguindo uma parte dos passos anteriores vamos obter a equação

$$
\left[\frac{1}{p} \sqrt{\frac{p+2}{|\lambda|}} g^{\prime}\right]^{2}-\left[\sqrt{\frac{\alpha}{2}} \sqrt{\frac{p+2}{|\lambda|}} g\right]^{2}=1
$$

Logo,

$$
g(\xi)=\sqrt{\frac{2}{\alpha}} \sqrt{\frac{|\lambda|}{p+2}} \sinh \left[p \sqrt{\frac{\alpha}{2}} \xi\right]
$$

mas $g(0)=0$, portanto, teremos uma singularidade em $\xi=0$ para $\varphi=\frac{1}{g^{\frac{2}{p}}}$.

\section{A.1.2 $q \neq 0$ e $\lambda>0$}

Queremos obter uma solução tipo standing-wave para a equação

$$
i u_{t}+\frac{1}{2} u_{x x}-q \delta(x) u+\lambda|u|^{p} u=0,
$$

$\operatorname{com} \lambda>0$, da forma

$$
u(x, t)=e^{i \omega t} \phi(x),
$$


onde $\omega>0$ e $\phi: \mathbb{R} \rightarrow \mathbb{R}$ satisfazendo

$$
\left\{\begin{array}{l}
-\omega \phi(x)+\frac{1}{2} \phi^{\prime \prime}(x)+\lambda|\phi(x)|^{p} \phi(x)=0, \text { para } x \neq 0 \\
\phi \in \mathscr{D}\left(H_{q}\right)
\end{array}\right.
$$

Logo, $\phi \in \mathscr{D}\left(H_{q}\right)$ é contínua em $x=0$ e $\phi^{\prime}(0+)-\phi^{\prime}(0-)=2 q \phi(0)$. Assim, para $x \neq 0$ e considerando $\phi>0$, temos

$$
-\omega \phi(x)+\frac{1}{2} \phi^{\prime \prime}(x)+\lambda \phi^{p+1}(x)=0 .
$$

Se multiplicarmos a equação (A.4) por $\phi^{\prime}(x)$, pois $x \neq 0$, teremos

$$
\frac{d}{d x}\left[-\frac{\omega}{2} \phi^{2}(x)+\frac{1}{4}\left(\phi^{\prime}(x)\right)^{2}+\frac{\lambda}{p+2} \phi^{p+2}(x)\right]=0,
$$

$\log 0$

$$
-\frac{\omega}{2} \phi^{2}(x)+\frac{1}{4}\left(\phi^{\prime}(x)\right)^{2}+\frac{\lambda}{p+2} \phi^{p+2}(x)=0
$$

ou seja,

$$
\left(\phi^{\prime}(x)\right)^{2}=2 \omega \phi^{2}(x)-\frac{4 \lambda}{p+2} \phi^{p+2}(x)=\phi^{2}(x)\left(2 \omega-\frac{4 \lambda}{p+2} \phi^{p}(x)\right) .
$$

Como $\phi>0$,

$$
\frac{d \phi}{ \pm \phi \sqrt{2 \omega-\frac{4 \lambda}{p+2} \phi^{p}}}=d x
$$

e, pela equação (A.5), $\phi \leq\left(\omega \frac{p+2}{2 \lambda}\right)^{\frac{1}{p}}$; isto é, $\phi$ é limitada. Integrando (A.6), obtemos

$$
\int \frac{d \phi}{ \pm \phi \sqrt{2 \omega-\frac{4 \lambda}{p+2} \phi^{p}}}=x+c ; \quad c \text { constante. }
$$

Fazendo a mudança de variável $\phi=\left(\omega \frac{p+2}{2 \lambda}\right)^{\frac{1}{p}} \operatorname{sech}^{\frac{2}{p}}(\theta)$, assim

$$
d \phi=-\left(\omega \frac{p+2}{2 \lambda}\right)^{\frac{1}{p}} \frac{2}{p} \operatorname{sech}^{\frac{2}{p}}(\theta) \tanh (\theta) d \theta
$$

$\mathrm{e}$

$$
\phi \sqrt{2 \omega-\frac{4 \lambda}{p+2} \phi^{p}}=\sqrt{2 \omega}\left(\omega \frac{p+2}{2 \lambda}\right)^{\frac{1}{p}} \operatorname{sech}^{\frac{2}{p}}(\theta) \tanh (\theta) .
$$

Substituindo em (A.7), concluímos que

$$
\pm \theta=\frac{p \sqrt{2 \omega}}{2} x+d ; \quad d \text { constante }
$$


Como sech é uma função par, então

$$
\phi(x)=\left(\omega \frac{p+2}{2 \lambda}\right)^{\frac{1}{p}} \operatorname{sech}^{\frac{2}{p}}\left(\frac{p \sqrt{2 \omega}}{2} x+d\right) .
$$

Para que $\phi$ satisfaça a condição de salto $\phi^{\prime}(0+)-\phi^{\prime}(0-)=2 q \phi(0)$, então

$$
\phi(x)=\left(\omega \frac{p+2}{2 \lambda}\right)^{\frac{1}{p}} \operatorname{sech}^{\frac{2}{p}}\left(\frac{p \sqrt{2 \omega}}{2}|x|+d\right) .
$$

A seguir determinaremos $d$. Veja,

$$
\phi^{\prime}(x)= \begin{cases}-\sqrt{2 \omega}\left(\omega \frac{p+2}{2 \lambda}\right)^{\frac{1}{p}} \operatorname{sech}^{\frac{2}{p}}\left(\frac{p \sqrt{2 \omega}}{2}|x|+d\right) \tanh \left(\frac{p \sqrt{2 \omega}}{2}|x|+d\right), & x>0 \\ \sqrt{2 \omega}\left(\omega \frac{p+2}{2 \lambda}\right)^{\frac{1}{p}} \operatorname{sech}^{\frac{2}{p}}\left(\frac{p \sqrt{2 \omega}}{2}|x|+d\right) \tanh \left(\frac{p \sqrt{2 \omega}}{2}|x|+d\right), & x<0\end{cases}
$$

logo, desde que $\omega>\frac{q^{2}}{2}$ e do fato que $\psi^{\prime}(0+)-\psi^{\prime}(0-)=2 q \phi(0)$,

$$
-2 \sqrt{2 \omega} \tanh (d)=2 q \Rightarrow d=\tanh ^{-1}\left(-\frac{q}{\sqrt{2 \omega}}\right) .
$$

Portanto,

$$
u(x, t)=e^{i \omega t}\left[\sqrt{\omega \frac{p+2}{2 \lambda}} \operatorname{sech}\left(\frac{p \sqrt{2 \omega}}{2}|x|+\tanh ^{-1}\left(-\frac{q}{\sqrt{2 \omega}}\right)\right)\right]^{\frac{2}{p}},
$$

desde que $\omega>\frac{q^{2}}{2}$.

\section{A.1.3 $q \neq 0$ e $\lambda<0$}

Com $\lambda<0$, de forma análoga ao feito anteriormente,

$$
\phi(x)=\left(\omega \frac{p+2}{2|\lambda|}\right)^{\frac{1}{p}} \operatorname{cossech}^{\frac{2}{p}}\left(\frac{p \sqrt{2 \omega}}{2}|x|+d\right) .
$$

resolve a equação (A.2), desde que $d>0$, pois cossech tem uma singularidade em $x=0$. Então, do fato que $\phi^{\prime}(0+)-\phi^{\prime}(0-)=2 q \phi(0)$,

$$
-2 \sqrt{2 \omega} \operatorname{cotanh}(d)=2 q \Rightarrow \tanh (d)=-\frac{\sqrt{2 \omega}}{q} \Rightarrow d=\tanh ^{-1}\left(-\frac{\sqrt{2 \omega}}{q}\right) \stackrel{d>0}{\Rightarrow} d=\tanh ^{-1}\left(\frac{\sqrt{2 \omega}}{|q|}\right) .
$$

Portanto, para $\lambda<0$,

$$
u(x, t)=e^{i \omega t}\left[\sqrt{\omega \frac{p+2}{2|\lambda|}} \operatorname{cossech}\left(\frac{p \sqrt{2 \omega}}{2}|x|+\tanh ^{-1}\left(\frac{\sqrt{2 \omega}}{|q|}\right)\right)\right]^{\frac{2}{p}}
$$


desde que $\omega<\frac{q^{2}}{2}$.

\section{A.1.4 Casos particulares}

Afim de deixarmos claro como é o perfil da solução da NLS- $\delta$, vamos considerar valores para $p$ e $q$ nos perfis obtidos nas seções A.1.2 e A.1.3 desse apêndice, como uma breve ilustração. Consideremos $p=2$. Então, se

- $\lambda=4$ e $q=2$, consideraremos $\omega=8$, então

$$
\phi(x)=2 \operatorname{sech}\left(4|x|+\tanh ^{-1}\left(-\frac{1}{2}\right)\right),
$$

cujo gráfico é

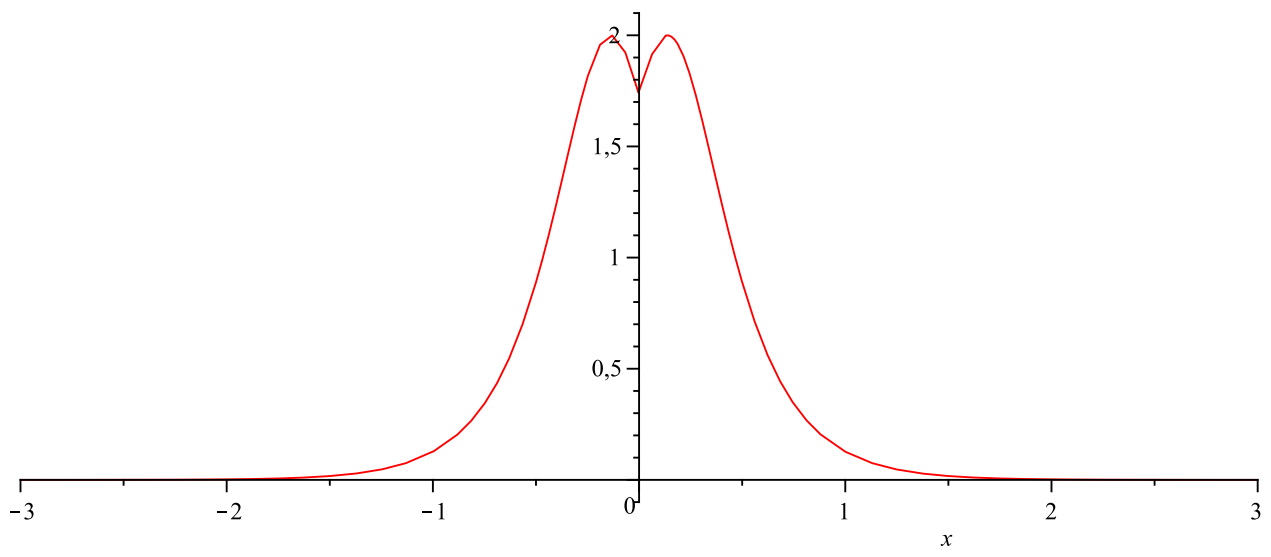

Figura A.1: Perfil $\operatorname{com} \lambda>0$ e $q>0$.

- $\lambda=4$ e $q=-2$, também consideraremos $\omega=8$, então

$$
\phi(x)=2 \operatorname{sech}\left(4|x|+\tanh ^{-1}\left(\frac{1}{2}\right)\right),
$$

cujo gráfico é

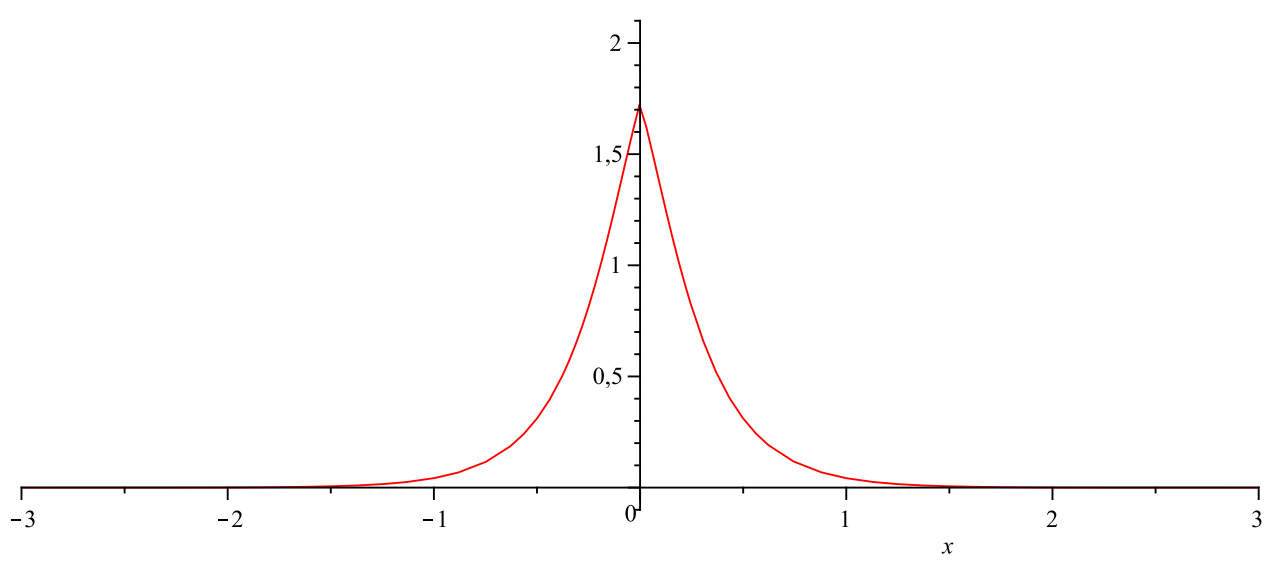

Figura A.2: Perfil $\operatorname{com} \lambda>0$ e $q<0$. 
- $\lambda=-4$ e $q=2$ ou $q=-2$, consideraremos $\omega=1$, então

$$
\phi(x)=\frac{\sqrt{2}}{2} \operatorname{cossech}\left(\sqrt{2}|x|+\tanh ^{-1}\left(\frac{\sqrt{2}}{2}\right)\right),
$$

cujo gráfico é

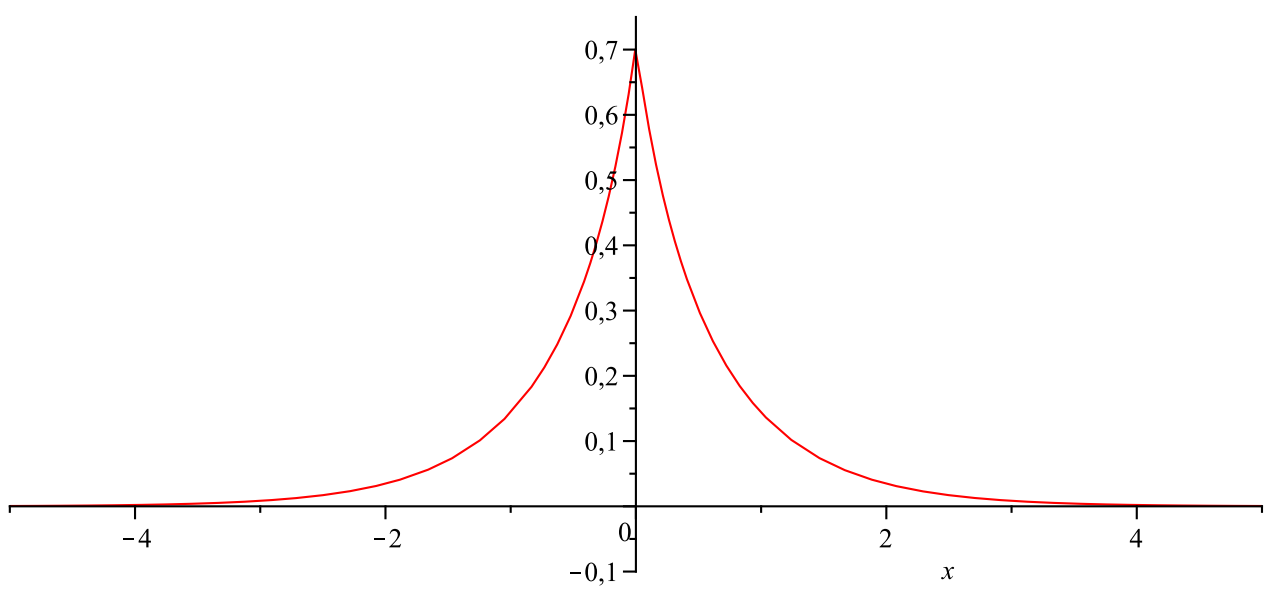

Figura A.3: Perfil com $\lambda>0$ e $q>0$ ou $q<0$. 


\section{Contas do Teorema 6.1.1}

Nesse Apêndice apresentamos cálculos que foram omitido da demostração do Teorema 6.1.1.

\section{B.1 Derivadas de $h_{j}$}

Nessa seção apresentamos as derivadas de $h_{j}$ com relação à $\lambda$ de ordem um e dois nas quatro partes onde $h_{j}$ está definida.

$1^{\circ}$ Caso: $x \geq 0$ e $y \geq 0$ :

$$
\begin{aligned}
2 \pi \frac{d h_{j}}{d \lambda}(\lambda, x, y)= & \chi_{j}^{\prime}(\lambda) \frac{\sqrt{2 \lambda} \cos (\sqrt{2 \lambda}(x-y))}{2 \lambda+q^{2}}+\chi_{j}(\lambda) \frac{\cos (\sqrt{2 \lambda}(x-y))}{\sqrt{2 \lambda}\left(2 \lambda+q^{2}\right)} \\
& -2 \chi_{j}(\lambda) \frac{\sqrt{2 \lambda} \cos (\sqrt{2 \lambda}(x-y))}{\left(2 \lambda+q^{2}\right)^{2}}-\chi_{j}(\lambda) \frac{\operatorname{sen}(\sqrt{2 \lambda}(x-y))}{2 \lambda+q^{2}}(x-y)
\end{aligned}
$$

e

$$
\begin{aligned}
2 \pi \frac{d^{2} h_{j}}{d \lambda^{2}}(\lambda, x, y)= & \chi_{j}^{\prime \prime}(\lambda) \frac{\sqrt{2 \lambda} \cos (\sqrt{2 \lambda}(x-y))}{2 \lambda+q^{2}}+2 \chi_{j}^{\prime}(\lambda) \frac{\cos (\sqrt{2 \lambda}(x-y))}{\sqrt{2 \lambda}\left(2 \lambda+q^{2}\right)} \\
& -4 \chi_{j}^{\prime}(\lambda) \frac{\sqrt{2 \lambda} \cos (\sqrt{2 \lambda}(x-y))}{\left(2 \lambda+q^{2}\right)^{2}}-2 \chi_{j}^{\prime}(\lambda) \frac{\operatorname{sen}(\sqrt{2 \lambda}(x-y))}{2 \lambda+q^{2}}(x-y) \\
& -\chi_{j}(\lambda) \frac{\cos (\sqrt{2 \lambda}(x-y))}{2 \sqrt{2}\left(2 \lambda+q^{2}\right) \lambda^{3 / 2}}-4 \chi_{j}(\lambda) \frac{\cos (\sqrt{2 \lambda}(x-y))}{\sqrt{2 \lambda}\left(2 \lambda+q^{2}\right)^{2}} \\
& -\chi_{j}(\lambda) \frac{\operatorname{sen}(\sqrt{2 \lambda}(x-y))}{2 \lambda\left(2 \lambda+q^{2}\right)}(x-y)+8 \chi_{j}(\lambda) \frac{\sqrt{2 \lambda} \cos (\sqrt{2 \lambda}(x-y))}{\left(2 \lambda+q^{2}\right)^{3}} \\
& +4 \chi_{j}(\lambda) \frac{\operatorname{sen}(\sqrt{2 \lambda}(x-y))}{\left(2 \lambda+q^{2}\right)^{2}}(x-y)-\chi_{j}(\lambda) \frac{\cos (\sqrt{2 \lambda}(x-y))}{\sqrt{2 \lambda}\left(2 \lambda+q^{2}\right)}(x-y)^{2} .
\end{aligned}
$$

2o Caso: $x \geq 0$ e $y \leq 0$ :

$$
2 \pi \frac{d h_{i}}{d \lambda}(\lambda, x, y)=\frac{\chi_{i}^{\prime}(\lambda) \sqrt{2 \lambda} \cos (\sqrt{2 \lambda}(x-y))}{2 \lambda+q^{2}}+\frac{\chi_{i}(\lambda) \cos (\sqrt{2 \lambda}(x-y))}{\sqrt{2 \lambda}\left(2 \lambda+q^{2}\right)}
$$




$$
\begin{aligned}
& -\frac{\chi_{i}(\lambda) \operatorname{sen}(\sqrt{2 \lambda}(x-y))}{2 \lambda+q^{2}}(x-y)-2 \frac{\chi_{i}(\lambda) \sqrt{2 \lambda} \cos (\sqrt{2 \lambda}(x-y))}{\left(2 \lambda+q^{2}\right)^{2}} \\
& +q \frac{\chi_{i}^{\prime}(\lambda) \operatorname{sen}(\sqrt{2 \lambda}(x-y))}{2 \lambda+q^{2}}+q \frac{\chi_{i}(\lambda) \cos (\sqrt{2 \lambda}(x-y))}{\sqrt{2 \lambda}\left(2 \lambda+q^{2}\right)}(x-y) \\
& -2 q \frac{\chi_{i}(\lambda) \operatorname{sen}(\sqrt{2 \lambda}(x-y))}{\left(2 \lambda+q^{2}\right)^{2}}+\frac{\chi_{i}^{\prime}(\lambda) \sqrt{2 \lambda} \cos (\sqrt{2 \lambda}(x+y))}{2 \lambda+q^{2}} \\
& +\frac{\chi_{i}(\lambda) \cos (\sqrt{2 \lambda}(x+y))}{\sqrt{2 \lambda}\left(2 \lambda+q^{2}\right)}-2 \frac{\chi_{i}(\lambda) \sqrt{2 \lambda} \cos (\sqrt{2 \lambda}(x+y))}{\left(2 \lambda+q^{2}\right)^{2}}
\end{aligned}
$$

e

$$
\begin{aligned}
& 2 \pi \frac{d^{2} h_{i}}{d \lambda^{2}}(\lambda, x, y)=2 \frac{\chi_{i}^{\prime}(\lambda) \cos (\sqrt{2 \lambda}(x-y))}{\sqrt{2 \lambda}\left(2 \lambda+q^{2}\right)}-\frac{\chi_{i}(\lambda) \cos (\sqrt{2 \lambda}(x-y))}{(2 \lambda)^{3 / 2}\left(2 \lambda+q^{2}\right)} \\
& -\frac{\chi_{i}(\lambda) \cos (\sqrt{2 \lambda}(x-y))}{\sqrt{2 \lambda}\left(2 \lambda+q^{2}\right)}(x-y)^{2}-q \frac{\chi_{i}(\lambda) \operatorname{sen}(\sqrt{2 \lambda}(x-y))}{2 \lambda\left(2 \lambda+q^{2}\right)}(x-y)^{2} \\
& +2 \frac{\chi_{i}^{\prime}(\lambda) \cos (\sqrt{2 \lambda}(x+y))}{\sqrt{2 \lambda}\left(2 \lambda+q^{2}\right)}-\frac{\chi_{i}(\lambda) \cos (\sqrt{2 \lambda}(x+y))}{(2 \lambda)^{3 / 2}\left(2 \lambda+q^{2}\right)} \\
& -2 \frac{\chi_{i}^{\prime}(\lambda) \operatorname{sen}(\sqrt{2 \lambda}(x-y))}{2 \lambda+q^{2}}(x-y)+4 \frac{\chi_{i}(\lambda) \operatorname{sen}(\sqrt{2 \lambda}(x-y))}{\left(2 \lambda+q^{2}\right)^{2}}(x-y) \\
& +q \frac{\chi_{i}^{\prime \prime}(\lambda) \operatorname{sen}(\sqrt{2 \lambda}(x-y))}{2 \lambda+q^{2}}-4 q \frac{\chi_{i}^{\prime}(\lambda) \operatorname{sen}(\sqrt{2 \lambda}(x-y))}{\left(2 \lambda+q^{2}\right)^{2}} \\
& +8 q \frac{\chi_{i}(\lambda) \operatorname{sen}(\sqrt{2 \lambda}(x-y))}{\left(2 \lambda+q^{2}\right)^{3}}+2 q \frac{\chi_{i}^{\prime}(\lambda) \cos (\sqrt{2 \lambda}(x-y))}{\sqrt{2 \lambda}\left(2 \lambda+q^{2}\right)}(x-y) \\
& -4 q \frac{\chi_{i}(\lambda) \cos (\sqrt{2 \lambda}(x-y))}{\sqrt{2 \lambda}\left(2 \lambda+q^{2}\right)^{2}}(x-y)+\frac{\chi_{i}^{\prime \prime}(\lambda) \sqrt{2 \lambda} \cos (\sqrt{2 \lambda}(x-y))}{2 \lambda+q^{2}} \\
& -4 \frac{\chi_{i}^{\prime}(\lambda) \sqrt{2 \lambda} \cos (\sqrt{2 \lambda}(x-y))}{\left(2 \lambda+q^{2}\right)^{2}}-\frac{\chi_{i}(\lambda) \operatorname{sen}(\sqrt{2 \lambda}(x-y))}{2 \lambda\left(2 \lambda+q^{2}\right)}(x-y) \\
& -4 \frac{\chi_{i}(\lambda) \cos (\sqrt{2 \lambda}(x-y))}{\sqrt{2 \lambda}\left(2 \lambda+q^{2}\right)^{2}}+8 \frac{\chi_{i}(\lambda) \sqrt{2 \lambda} \cos (\sqrt{2 \lambda}(x-y))}{\left(2 \lambda+q^{2}\right)^{3}} \\
& +\frac{\chi_{i}^{\prime \prime}(\lambda) \sqrt{2 \lambda} \cos (\sqrt{2 \lambda}(x+y))}{2 \lambda+q^{2}}-4 \frac{\chi_{i}^{\prime}(\lambda) \sqrt{2 \lambda} \cos (\sqrt{2 \lambda}(x+y))}{\left(2 \lambda+q^{2}\right)^{2}} \\
& -4 \frac{\chi_{i}(\lambda) \cos (\sqrt{2 \lambda}(x+y))}{\sqrt{2 \lambda}\left(2 \lambda+q^{2}\right)^{2}}+8 \frac{\chi_{i}(\lambda) \sqrt{2 \lambda} \cos (\sqrt{2 \lambda}(x+y))}{\left(2 \lambda+q^{2}\right)^{3}} \\
& -q \frac{\chi_{i}(\lambda) \cos (\sqrt{2 \lambda}(x-y))}{(2 \lambda)^{3 / 2}\left(2 \lambda+q^{2}\right)}(x-y) \text {. }
\end{aligned}
$$

$3^{\circ}$ Caso: $x \leq 0$ e $y \geq 0$ : A menos de sinal é como do caso anterior. 
4. Caso: $x \leq 0$ e $y \leq 0$ :

$$
\begin{aligned}
2 \pi \frac{d}{d \lambda} h_{i}(\lambda, x, y)= & \chi_{i}^{\prime}(\lambda) \frac{\cos (\sqrt{2 \lambda}(x-y))}{\sqrt{2 \lambda}} \underbrace{-\chi_{i}(\lambda) \frac{\cos (\sqrt{2 \lambda}(x-y))}{(2 \lambda)^{3 / 2}}}_{J_{1}} \\
& -\chi_{i}(\lambda) \frac{\operatorname{sen}(\sqrt{2 \lambda}(x-y))}{2 \lambda}(x-y)-\frac{\chi_{i}^{\prime}(\lambda)}{2 \lambda+q^{2}}[2 q \operatorname{sen}(\sqrt{2 \lambda}(x+y))] \\
& +4 q \chi_{i}(\lambda) \frac{\operatorname{sen}(\sqrt{2 \lambda}(x+y))}{\left(2 \lambda+q^{2}\right)^{2}}-2 q \chi_{i}(\lambda) \frac{\cos (\sqrt{2 \lambda}(x+y))}{\sqrt{2 \lambda}\left(2 \lambda+q^{2}\right)}(x+y) \\
& -\frac{\chi_{i}^{\prime}(\lambda)}{\sqrt{2 \lambda}\left(2 \lambda+q^{2}\right)}\left[2 q^{2} \cos (\sqrt{2 \lambda}(x+y))\right]+\underbrace{\chi_{i}(\lambda) \frac{2 q^{2} \cos (\sqrt{2 \lambda}(x+y))}{(2 \lambda)^{3 / 2}\left(2 \lambda+q^{2}\right)}}_{J_{2}} \\
& +\chi_{i}(\lambda) \frac{4 q^{2} \cos (\sqrt{2 \lambda}(x+y))}{\sqrt{2 \lambda}\left(2 \lambda+q^{2}\right)^{2}}+\chi_{i}(\lambda) \frac{2 q^{2} \operatorname{sen}(\sqrt{2 \lambda}(x+y))}{2 \lambda\left(2 \lambda+q^{2}\right)^{2}}(x+y) \\
& +\chi_{i}^{\prime}(\lambda) \frac{q^{2}}{\sqrt{2 \lambda}\left(2 \lambda+q^{2}\right)} \cos (\sqrt{2 \lambda}(x-y))-\underbrace{\chi_{J_{3}}}_{J_{i}(\lambda) \frac{q^{2} \cos (\sqrt{2 \lambda}(x-y))}{(2 \lambda)^{3 / 2}\left(2 \lambda+q^{2}\right)}} \\
& -2 \chi_{i}(\lambda) \frac{q^{2} \cos (\sqrt{2 \lambda}(x-y))}{\sqrt{2 \lambda}\left(2 \lambda+q^{2}\right)^{2}}-\chi_{i}(\lambda) \frac{q^{2} \operatorname{sen}(\sqrt{2 \lambda}(x-y))}{2 \lambda\left(2 \lambda+q^{2}\right)^{2}}(x-y)
\end{aligned}
$$

e

$$
\begin{aligned}
2 \pi \frac{d^{2}}{d^{2} \lambda} h_{i}(\lambda, x, y)= & \chi_{i}^{\prime \prime}(\lambda) \frac{\cos (\sqrt{2 \lambda}(x-y))}{\sqrt{2 \lambda}}-2 \chi_{i}^{\prime}(\lambda) \frac{\cos (\sqrt{2 \lambda}(x-y))}{(2 \lambda)^{3 / 2}} \\
& -2 \chi_{i}^{\prime}(\lambda) \frac{\operatorname{sen}(\sqrt{2 \lambda}(x-y))}{2 \lambda}(x-y)+3 \chi_{i}(\lambda) \frac{\cos (\sqrt{2 \lambda}(x-y))}{(2 \lambda)^{5 / 2}} \\
& +3 \chi_{i}(\lambda) \frac{\operatorname{sen}(\sqrt{2 \lambda}(x-y))}{(2 \lambda)^{2}}(x-y)-\chi_{i}(\lambda) \frac{\cos (\sqrt{2 \lambda}(x-y))}{(2 \lambda)^{3 / 2}}(x-y)^{2} \\
& -\frac{\chi_{i}^{\prime \prime}(\lambda)}{2 \lambda+q^{2}}[2 q \operatorname{sen}(\sqrt{2 \lambda}(x+y))]+8 q \chi_{i}^{\prime}(\lambda) \frac{\operatorname{sen}(\sqrt{2 \lambda}(x+y))}{\left(2 \lambda+q^{2}\right)^{2}} \\
& -4 q \chi_{i}^{\prime}(\lambda) \frac{\cos (\sqrt{2 \lambda}(x+y))}{\sqrt{2 \lambda}\left(2 \lambda+q^{2}\right)}(x+y) \\
& -16 q \chi_{i}(\lambda) \frac{\operatorname{sen}(\sqrt{2 \lambda}(x+y))}{\left(2 \lambda+q^{2}\right)^{3}}+8 q \chi_{i}(\lambda) \frac{\cos (\sqrt{2 \lambda}(x+y))}{\sqrt{2 \lambda}\left(2 \lambda+q^{2}\right)^{2}}(x+y) \\
& +2 q \chi_{i}(\lambda) \frac{\cos (\sqrt{2 \lambda}(x+y))}{(2 \lambda)^{3 / 2}\left(2 \lambda+q^{2}\right)}(x+y)+2 q \chi_{i}(\lambda) \frac{\operatorname{sen}(\sqrt{2 \lambda}(x+y))}{2 \lambda\left(2 \lambda+q^{2}\right)}(x+y)^{2} \\
& -\frac{\chi_{i}^{\prime \prime}(\lambda)}{\sqrt{2 \lambda}\left(2 \lambda+q^{2}\right)}\left[2 q^{2} \cos (\sqrt{2 \lambda}(x+y))\right]+\chi_{i}^{\prime}(\lambda) \frac{4 q^{2} \cos (\sqrt{2 \lambda}(x+y))}{(2 \lambda)^{3 / 2}\left(2 \lambda+q^{2}\right)}
\end{aligned}
$$




$$
\begin{aligned}
& +\chi_{i}^{\prime}(\lambda) \frac{8 q^{2} \cos (\sqrt{2 \lambda}(x+y))}{\sqrt{2 \lambda}\left(2 \lambda+q^{2}\right)^{2}}+\chi_{i}^{\prime}(\lambda) \frac{4 q^{2} \operatorname{sen}(\sqrt{2 \lambda}(x+y))}{2 \lambda\left(2 \lambda+q^{2}\right)}(x+y) \\
& -6 q^{2} \frac{\cos (\sqrt{2 \lambda}(x+y))}{(2 \lambda)^{5 / 2}\left(2 \lambda+q^{2}\right)}-8 q^{2} \chi_{i}(\lambda) \frac{\cos (\sqrt{2 \lambda}(x+y))}{(2 \lambda)^{3 / 2}\left(2 \lambda+q^{2}\right)^{2}} \\
& -6 q^{2} \chi_{i}(\lambda) \frac{\operatorname{sen}(\sqrt{2 \lambda}(x+y))}{(2 \lambda)^{2}\left(2 \lambda+q^{2}\right)}(x+y)-16 q^{2} \chi_{i}(\lambda) \frac{\cos (\sqrt{2 \lambda}(x+y))}{\sqrt{2 \lambda}\left(2 \lambda+q^{2}\right)^{3}} \\
& -8 q^{2} \chi_{i}(\lambda) \frac{\operatorname{sen}(\sqrt{2 \lambda}(x+y))}{2 \lambda\left(2 \lambda+q^{2}\right)^{2}}(x+y)+2 q^{2} \chi_{i}(\lambda) \frac{\cos (\sqrt{2 \lambda}(x+y))}{(2 \lambda)^{3 / 2}\left(2 \lambda+q^{2}\right)}(x+y)^{2} \\
& +\chi_{i}^{\prime \prime}(\lambda) \frac{q^{2}}{\sqrt{2 \lambda}\left(2 \lambda+q^{2}\right)} \cos (\sqrt{2 \lambda}(x-y))-2 \chi_{i}^{\prime}(\lambda) \frac{q^{2} \cos (\sqrt{2 \lambda}(x-y))}{(2 \lambda)^{3 / 2}\left(2 \lambda+q^{2}\right)} \\
& -4 \chi_{i}^{\prime}(\lambda) \frac{q^{2} \cos (\sqrt{2 \lambda}(x-y))}{\sqrt{2 \lambda}\left(2 \lambda+q^{2}\right)^{2}}-2 \chi_{i}^{\prime}(\lambda) \frac{q^{2} \operatorname{sen}(\sqrt{2 \lambda}(x-y))}{2 \lambda\left(2 \lambda+q^{2}\right)}(x-y) \\
& +3 q^{2} \chi_{i}(\lambda) \frac{\cos (\sqrt{2 \lambda}(x-y))}{(2 \lambda)^{5 / 2}\left(2 \lambda+q^{2}\right)}+4 q^{2} \chi_{i}(\lambda) \frac{\cos (\sqrt{2 \lambda}(x-y))}{(2 \lambda)^{3 / 2}\left(2 \lambda+q^{2}\right)^{2}} \\
& +3 q^{2} \chi_{i}(\lambda) \frac{\operatorname{sen}(\sqrt{2 \lambda}(x-y))}{(2 \lambda)^{2}\left(2 \lambda+q^{2}\right)}(x-y)+8 q^{2} \chi_{i}(\lambda) \frac{\cos (\sqrt{2 \lambda}(x-y))}{\sqrt{2 \lambda}\left(2 \lambda+q^{2}\right)^{3}} \\
& +4 \chi_{i}(\lambda) \frac{q^{2} \operatorname{sen}(\sqrt{2 \lambda}(x-y))}{2 \lambda\left(2 \lambda+q^{2}\right)^{2}}(x-y)-q^{2} \chi_{i}(\lambda) \frac{\cos (\sqrt{2 \lambda}(x-y))}{(2 \lambda)^{3 / 2}\left(2 \lambda+q^{2}\right)}(x-y)^{2} .
\end{aligned}
$$

\section{B.2 Limitação em $t$ no $1^{\circ}$ Caso}

Considere $a(t)=2 \pi \sqrt{t}-2 \sqrt{2 \pi} t+2 t^{3 / 2} \ln \left(1+\sqrt{\frac{2 \pi}{t}}\right)$. Note que, chamando $s=\frac{2 \pi}{t}$, então

$$
\lim _{t \rightarrow 0} t^{3 / 2} \ln \left(1+\sqrt{\frac{2 \pi}{t}}\right)=\lim _{s \rightarrow+\infty}(2 \pi)^{3 / 2} \frac{\ln (1+\sqrt{s})}{s^{3 / 2}}
$$

usando L'Hopital teremos

$$
\lim _{t \rightarrow 0} t^{3 / 2} \ln \left(1+\sqrt{\frac{2 \pi}{t}}\right)=\lim _{s \rightarrow+\infty}(2 \pi)^{3 / 2} \frac{1}{3 s(1+\sqrt{s})}=0
$$

$\operatorname{assim} a(0)=0, \operatorname{logo} a$ é contínua para todo $t \geq 0$. Vejamos quanto $t \rightarrow \infty$. Assim

$$
\lim _{t \rightarrow \infty} a(t)=\lim _{s \rightarrow 0}(2 \pi)^{3 / 2} \frac{s-2 \sqrt{s}+2 \ln (1+\sqrt{s})}{s^{3 / 2}},
$$

usando L'Hopital teremos

$$
\lim _{t \rightarrow \infty} a(t)=\lim _{s \rightarrow 0} \frac{2(2 \pi)^{3 / 2}}{3} \frac{1}{1+\sqrt{s}}=\frac{2(2 \pi)^{3 / 2}}{3}=\frac{4 \sqrt{2}}{3}(\pi)^{3 / 2}
$$


Como $a$ é crescente para $t \geq 0$, então

$$
|a(t)| \leq \frac{4 \sqrt{2}}{3}(\pi)^{3 / 2}
$$

\section{B.3 Integrais finitas do primeiro caso}

Nessa seção, apresentamos que as integrais do $\mathbf{1}^{\circ}$ Caso são limitadas na demonstração do Teorema 6.1.1.

$$
\begin{gathered}
\int_{0}^{\infty}\left|\chi_{2}^{\prime \prime}(\lambda)\right| \frac{\sqrt{2 \lambda}}{2 \lambda+q^{2}} d \lambda=\int_{1}^{2}\left|\chi_{2}^{\prime \prime}(\lambda)\right| \frac{\sqrt{2 \lambda}}{2 \lambda+q^{2}} d \lambda<\infty \\
\int_{0}^{\infty}\left|\chi_{2}^{\prime}(\lambda)\right| \frac{1}{\sqrt{2 \lambda}\left(2 \lambda+q^{2}\right)} d \lambda=\int_{1}^{2}\left|\chi_{2}^{\prime}(\lambda)\right| \frac{1}{\sqrt{2 \lambda}\left(2 \lambda+q^{2}\right)} d \lambda<\infty \\
\int_{0}^{\infty}\left|\chi_{2}^{\prime}(\lambda)\right| \frac{\sqrt{2 \lambda}}{\left(2 \lambda+q^{2}\right)^{2}} d \lambda=\int_{1}^{2}\left|\chi_{2}^{\prime}(\lambda)\right| \frac{\sqrt{2 \lambda}}{\left(2 \lambda+q^{2}\right)^{2}} d \lambda<\infty \\
\int_{0}^{\infty}\left|\chi_{2}^{\prime}(\lambda)\right| \frac{1}{2 \lambda+q^{2}} d \lambda=\int_{1}^{2}\left|\chi_{2}^{\prime}(\lambda)\right| \frac{1}{2 \lambda+q^{2}} d \lambda<\infty \\
\int_{0}^{\infty}\left|\chi_{2}(\lambda)\right| \frac{1}{2 \sqrt{2}\left(2 \lambda+q^{2}\right) \lambda^{3 / 2}} d \lambda \leq c \int_{1}^{\infty} \frac{1}{\lambda^{5 / 2}} d \lambda<\infty \\
\int_{0}^{\infty}\left|\chi_{2}(\lambda)\right| \frac{1}{\sqrt{2 \lambda}\left(2 \lambda+q^{2}\right)^{2}} d \lambda \leq c \int_{1}^{\infty} \frac{1}{\lambda^{5 / 2}} d \lambda<\infty \\
\int_{0}^{\infty}\left|\chi_{2}(\lambda)\right| \frac{1}{2 \lambda\left(2 \lambda+q^{2}\right)} d \lambda \leq c \int_{1}^{\infty} \frac{1}{(2 \lambda)^{2}} d \lambda<\infty \\
\int_{0}^{\infty}\left|\chi_{2}(\lambda)\right| \frac{1}{\left(2 \lambda+q^{2}\right)^{2}} d \lambda \leq c \int_{1}^{\infty} \frac{1}{(2 \lambda)^{2}} d \lambda<\infty \\
\int_{0}^{\infty}\left|\chi_{2}(\lambda)\right| \frac{\sqrt{2 \lambda}}{\left(2 \lambda+q^{2}\right)^{3}} d \lambda \leq c \int_{1}^{\infty} \frac{1}{(2 \lambda)^{5 / 2}} d \lambda<\infty \\
\int_{0}^{\infty}\left|\chi_{2}(\lambda)\right| \frac{1}{\left(2 \lambda+q^{2}\right)^{2}} d \lambda \leq c \int_{1}^{\infty} \frac{1}{(2 \lambda)^{2}} d \lambda<\infty \\
\int_{0}^{\infty}(\lambda) \mid \frac{1}{\sqrt{2 \lambda}\left(2 \lambda+q^{2}\right)} d \lambda \leq c \int_{1}^{\infty} \frac{1}{(2 \lambda)^{3 / 2}} d \lambda<\infty
\end{gathered}
$$

\section{B.4 Lema de Schur}

Suponhamos que $K(x, y)$ é uma função localmente integrável sobre o produto de dois espaços de medida $\sigma$-finita $(X, \mu) \times(Y, \nu)$ e seja $T$ um operador linear dado por

$$
T(f)(x)=\int_{Y} K(x, y) f(y) d \nu(y),
$$

onde $f$ é limitada com suporte compacto. É uma simples consequência do teorema de Fubini que para quase todo $x \in X$ a integral definida $T$ converge absolutamente. O seguinte lema fornece um 
critério suficiente para a limitação $L^{p}$ de $T$.

Lema B.4.1 (Critério de Schur). Suponhamos que uma função localmente integrável $K(x, y)$ satisfaz

$$
\begin{aligned}
& \sup _{x \in X} \int_{Y}|K(x, y)| d \nu(y)=A<\infty, \\
& \sup _{y \in Y} \int_{X}|K(x, y)| d \mu(x)=B<\infty .
\end{aligned}
$$

Então, o operador $T$ estende-se para um operador de $L^{p}(Y)$ para $L^{p}(X)$ com norma $A^{1-\frac{1}{p}} B^{\frac{1}{p}}$ para $1 \leq p \leq \infty$

Para mais detalhes veja [21]. 


\section{Referências}

[1] Adams, R. Sobolev Spaces. Pure and applied mathematics. Academic Press, 1975. 90

[2] Agrawal, G. Nonlinear Fiber Optics. Academic Press. Academic Press, 2013. 2

[3] Albeverio, S.; Gesztesy, F.; Høegh-Krohn, R.; Holden, H. Solvable Models in Quantum Mechanics, Segunda ed. AMS Chelsea Publishing Series. AMS Chelsea Pub., 2005. 9, 10, $12,13,92,93,94$

[4] Albeverio, S.; Kurasov, P. Singular perturbations of differential operators. Solvable Schrödinger type operators. Cambridge: Cambridge University Press, 1999. 10

[5] Angulo, J. P.; Ferreira, L. C. F. On the Schrödinger equation with singular potentials. arXiv preprint arXiv:130\%.6895 (Jul 2013). 31, 94

[6] Angulo, J. P.; Ponce, G. The non-linear Schrödinger equation with a periodic $\delta$-interaction. Bull. Braz. Math. Soc. (N.S.) 44, 3 (2013), 497-551. 10

[7] Baudouin, L.; Kavian, O.; Puel, J.-P. Regularity for a Schrödinger equation with singular potentials and application to bilinear optimal control . Journal of Differential Equations 216, 1 (2005), $188-222.1$

[8] Berezin, F. A.; Shubin, M. A. The Schrödinger equation. Transl. from the Russian by Yu. Rajabov, D. A. Leites and N. A. Sakharova. Rev. and exp. version., rev. and exp. version ed. Dordrecht etc.: Kluwer Academic Publishers, 1991. 87

[9] Carr, J. Applications of Centre Manifold Theory. Applied Mathematical Sciences. SpringerVerlag, 1981. 24

[10] Caudrelier, V.; Mintchev, M.; Ragoucy, E. Solving the quantum nonlinear Schrödinger equation with $\delta$-type impurity. J. Math. Phys. 46, 4 (2005), 042703, 24. 3

[11] Cazenave, T. Semilinear Schrödinger Equations. Courant Lecture Notes in Mathematics. American Mathematical Society, 2003. 6, 25, 49, 87, 88

[12] Datchev, K.; Holmer, J. Fast soliton scattering by attractive delta impurities. Commun. Partial Differ. Equations 34, 9 (2009), 1074-1113. 2, 31, 33 
[13] Davis, K. B.; Mewes, M. O.; Andrews, M. R.; van Druten, N. J.; Durfee, D. S.; Kurn, D. M.; Ketterle, W. Bose-Einstein Condensation in a Gas of Sodium Atoms. Phys. Rev. Lett. 75 (Nov 1995), 3969-3973. 2

[14] Dolbeault, J.; Esteban, M. J.; Laptev, A.; Loss, M. One-dimensional GagliardoNirenberg-Sobolev inequalities: Remarks on duality and flows. arXiv preprint arXiv:1309.0987 (2013). 29

[15] Duchêne, V.; Marzuola, J. L.; Weinstein, M. I. Wave operator bounds for 1-dimensional Schrödinger operators with singular potentials and applications. Journal of Mathematical Physics 52, 1 (2011), 17. 6, 25, 26, 31, 32

[16] Evans, L. Partial Differential Equations. Graduate studies in mathematics. American Mathematical Society, 2010. 29, 46

[17] Fukuizumi, R.; Jeanjean, L. Stability of standing waves for a nonlinear Schrödinger equation with a repulsive Dirac delta potential. Discrete Contin. Dyn. Syst. 21, 1 (2008), 121-136. 4,40

[18] Fukuizumi, R.; Ohta, M.; Ozawa, T. Nonlinear Schrödinger equation with a point defect. Ann. Inst. Henri Poincaré, Anal. Non Linéaire 25, 5 (2008), 837-845. 4, 40

[19] Gill, T. S. Optical guiding of laser beam in nonuniform plasma. Pramana 55, 5-6 (2000), 835-842. 91

[20] Goodman, R. H.; Holmes, P. J.; Weinstein, M. I. Strong NLS soliton-defect interactions. Physica D 192, 3-4 (2004), 215-248. 2

[21] Grafakos, L. Classical Fourier analysis, 2nd ed. ed. New York, NY: Springer, 2008. 106

[22] Hansjörg KIELhÖFER. Bifurcation theory. An introduction with applications to partial differential equations. 2nd revised ed. Applied Mathematical Sciences 156. Berlin: Springer. viii, 398 p., 2012. 5, 16

[23] Haragus, M.; Iooss, G. Local Bifurcations, Center Manifolds, and Normal Forms in Infinite-Dimensional Dynamical Systems. Universitext. Springer, 2010. 36

[24] Hayashi, N.; Nakamitsu, K.; Tsutsumi, M. Nonlinear Schrödinger equations in weighted Sobolev spaces. Funkc. Ekvacioj, Ser. Int. 31, 3 (1988), 363-381. 50, 53, 54

[25] Hislop, P. D.; Sigal, I. M. Introduction to spectral theory. With applications to Schrödinger operators. New York, NY: Springer-Verlag, 1996. 42

[26] Holmer, J.; Marzuola, J.; Zworski, M. Fast soliton scattering by delta impurities. Commun. Math. Phys. 274, 1 (2007), 187-216. 2, 31, 33 
[27] Huziniro, A.; Hiroshi, E., Eds. Topics in the theory of Schrödinger operators. River Edge, NJ: World Scientific, 2004. 1

[28] Iorio, R. J. Tópicos na Teoria da Equação de Schrödinger. Instituto de Matemática Pura e Aplicada, 1987. 31, 32

[29] Kaminaga, M.; Ohta, M. Stability of standing waves for nonlinear Schrödinger equation with attractive delta potential and repulsive nonlinearity. Saitama Math. J. 26 (2009), 39-48. 5,40

[30] Le COZ, S.; Fukuizumi, R.; Fibich, G.; Ksherim, B.; Sivan, Y. Instability of bound states of a nonlinear Schrödinger equation with a Dirac potential. Physica D: Nonlinear Phenomena 23\%, 8 (2008), 1103 - 1128. 4

[31] Linares, F.; Ponce, G. Introduction to nonlinear dispersive equations. Universitext. New York, NY: Springer. xi, 256 p., 2009. 15

[32] Menyuk, C. R. Soliton robustness in optical fibers. J. Opt. Soc. Am. B 10, 9 (Sep 1993), 1585-1591. 2

[33] Moloney, J.; Newell, A. Nonlinear optics. Boulder, CO: Westview Press, 2004. 2

[34] Nirenberg, L. Topics in Nonlinear Functional Analysis. Courant Lecture Notes in Mathematics. Courant Institute, 2001. 5

[35] Pillet, C.-A.; WAyne, C. Invariant manifolds for a class of dispersive, Hamiltonian, partial differential equations. J. Differ. Equations 141, 2 (1997), 310-326. 1

[36] Reed, M.; Simon, B. Methods of modern mathematical physics. II: Fourier analysis, selfadjointness. Methods of Modern Mathematical Physics. New York - San Francisco - London: Academic Press. XV, 1975. 11

[37] Reed, M.; Simon, B. Methods of modern mathematical physics. IV: Analysis of operators. Methods of Modern Mathematical Physics. New York - San Francisco - London: Academic Press. XV, 1978. 12

[38] Rose, H. A.; Weinstein, M. I. On the bound states of the nonlinear Schrödinger equation with a linear potential. Physica D 30, 1-2 (1988), 207-218. 2

[39] Schrödinger, E. An Undulatory Theory of the Mechanics of Atoms and Molecules. Phys. Rev. 28 (Dec 1926), 1049-1070. 1

[40] Seaman, B. T.; Carr, L. D.; Holland, M. J. Effect of a potential step or impurity on the Bose-Einstein condensate mean field. Physical Review A 71 (3/2005 2005). 2

[41] Shankar, R. Principles of Quantum Mechanics. Springer London, Limited, 2012. 1 
[42] Soffer, A.; Weinstein, M. Multichannel nonlinear scattering for nonintegrable equations. Commun. Math. Phys. 133, 1 (1990), 119-146. 2

[43] Soffer, A.; Weinstein, M. I. Multichannel nonlinear scattering theory for nonintegrable equations. Integrable systems and applications, Proc. Workshop, Oléron/Fr. 1988, Lect. Notes Phys. 342, 312-327 (1989)., 1989. 2

[44] Triebel, H. Spaces of distributions with weights. Multipliers in $L_{p}$-spaces with weights. Math. Nachr. 78 (1977), 339-355. 52

[45] WedER, R. Center manifold for nonintegrable nonlinear Schrödinger equations on the line. 85

[46] Yajima, K. Schrödinger equations with time-dependent unbounded singular potentials. Rev. Math. Phys. 23, 8 (2011), 823-838. 1 


\section{Índice Remissivo}

Assintoticamente estável, 85

Autofunção $\psi_{0}$, vii, 14

Autovalor $E_{0}$, vii, 14

Bifurcação, 16

Bound-state, 38

Carga, 24

Caso

atrativo, 2

repulsivo, 2

Constante de acoplamento, 2

Constante de Planck, 1

Energia, 24

Espaço de Sobolev com peso, 49

Espectro

essencial $\left(\sigma_{\text {ess }}\right), 14$

ponto $\left(\sigma_{p}\right)$, vii, 14

Estimativa

de Strichartz, 49

Extensão de Operadores Simétricos, 11

Extensões Autoadjuntas, 11

Fórmula de Krein, 12

Fórmulas de Von Neumann, 11

Função onda posição espaço, 1

Função potencial

atrativa, 2

repulsiva, 2
Índece de Fredholm, 17

Índices de deficiência, 9

Mecânica Quântica, 1

Não-linearidade

atrativa (focusing), 2

repulsiva (defocusing), 2

Onda viajante

estável, 4

instável, 4

Ondas viajantes (standing waves), 3

Operador

Fredholm não-linear, 17

Ponto

de bifurcação, 20

Projeção

espectral contínua, 33, 65, 86

espectro discreto, 35

Relações de Completamento, 32

Subespaço gerado, vii

Subespaços de deficiência, 9

Teorema

de Crandall-Rabinowitz, 9, 20, 38

Teoria de Von Neumann, 11

Transformada de Fourier generalizada, 32 
Variedade Invariante Centro, 36, 38, 40, 41, 65, 76,85 